ORNL/TM-2003/218

\title{
Prediction of External Corrosion for Steel Cylinders-2003 Report
}

Rick Schmoyer

and

B. F. Lyon 
This report has been reproduced directly from the best available copy.

Available to DOE and DOE contractors from the Office of Scientific and Technical Information, P.O. Box 62, Oak Ridge, TN 37831; prices available from 845-576-8401, FTS 626-8401.

Available to the public from the National Technical Information Service, U.S. Department of Commerce, 5285 Port Royal Rd., Springfield, VA 22161.

This report was prepared as an account of work sponsored by an agency of the United States Government. Neither the United States Government nor any agency thereof, nor any of their employees, makes any warranty, express or implied, or assumes any legal liability or responsibility for the accuracy, completeness, or usefulness of any information, apparatus, product, or process disclosed, or represents that its use would not infringe privately owned rights. Reference herein to any specific commercial product, process, or service by trade name, trademark, manufacturer, or otherwise, does not necessarily constitute or imply its endorsement, recommendation, or favoring by the United States Government or any agency thereof. The views and opinions of authors expressed herein do not necessarily state or reflect those of the United States Government or any agency thereof. 
ORNL/TM-2003/218

\title{
PREDICTION OF EXTERNAL CORROSION FOR STEEL CYLINDERS-2003 REPORT
}

\author{
Rick Schmoyer \\ and \\ B. F. Lyon
}

Date Published: September 2003

\author{
Prepared for \\ U.S. Department of Energy \\ Office of Environmental Restoration and Waste Management \\ under budget and reporting code EW 20
}

\author{
Prepared by \\ OAK RIDGE NATIONAL LABORATORY \\ P.O. Box 2008 \\ Oak Ridge, Tennessee 37831-6285 \\ managed by \\ UT-Battelle, LLC \\ for the \\ U.S. DEPARTMENT OF ENERGY \\ under contract DE-AC05-00OR22725
}





\section{TABLE OF CONTENTS}

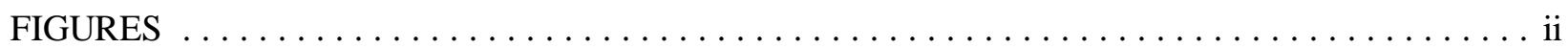

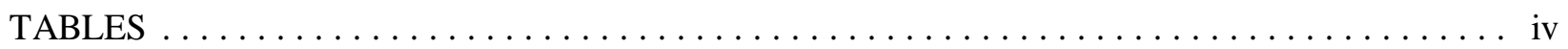

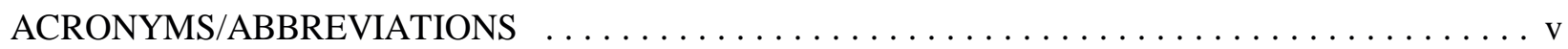

ACKNOWLEDGMENT $\ldots \ldots \ldots \ldots \ldots \ldots \ldots \ldots \ldots \ldots \ldots \ldots \ldots \ldots \ldots \ldots \ldots \ldots$

EXECUTIVE SUMMARY $\ldots \ldots \ldots \ldots \ldots \ldots \ldots \ldots \ldots \ldots \ldots \ldots \ldots \ldots \ldots$ vii

1. INTRODUCTION $\ldots \ldots \ldots \ldots \ldots \ldots \ldots \ldots \ldots \ldots \ldots \ldots \ldots \ldots \ldots \ldots \ldots$

2. APPROACHES TO MODELING CYLINDER WALL THICKNESS $\ldots \ldots \ldots \ldots \ldots \ldots \ldots \ldots$.

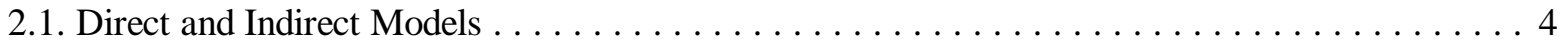

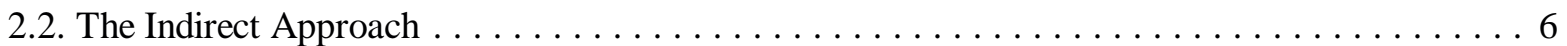

2.3. The Direct Approach .............................. 7

3. ULTRASONIC THICKNESS DATA $\ldots \ldots \ldots \ldots \ldots \ldots \ldots \ldots \ldots \ldots \ldots \ldots \ldots \ldots$

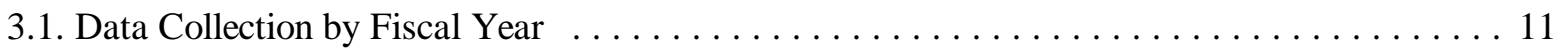

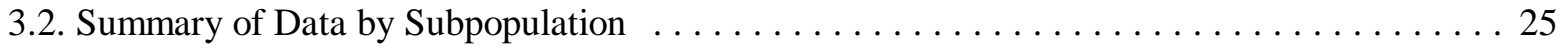

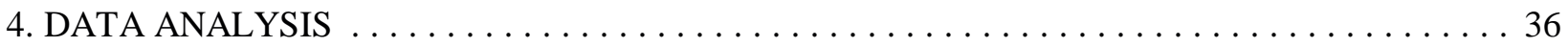

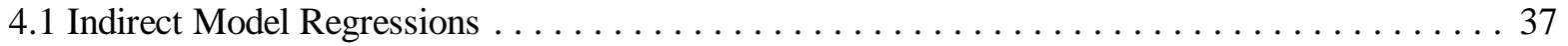

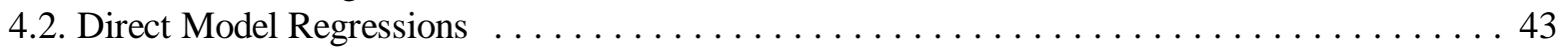

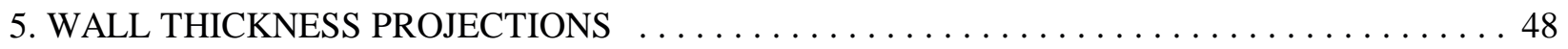

6. MODEL EVALUATION AND COMPARISON $\ldots \ldots \ldots \ldots \ldots \ldots \ldots \ldots \ldots \ldots \ldots \ldots$

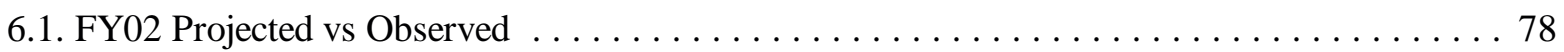

6.2. Comparison by Akaike Information Criterion $\ldots \ldots \ldots \ldots \ldots \ldots \ldots \ldots \ldots \ldots \ldots \ldots$

7. LIMITATIONS, CONCLUSIONS, AND RECOMMENDATIONS $\ldots \ldots \ldots \ldots \ldots \ldots \ldots$

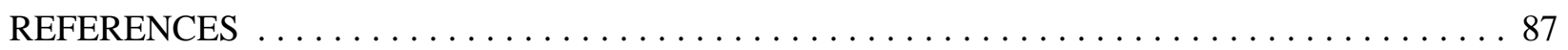

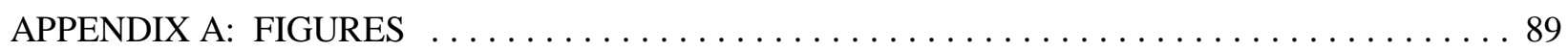

APPENDIX B: PROBABILITY AND CONFIDENCE BOUND

CALCULATIONS FOR THE INDIRECT MODEL $\ldots \ldots \ldots \ldots \ldots \ldots \ldots \ldots \ldots \ldots \ldots$

B.1. Cumulative distribution function for the difference of two distributions $\ldots \ldots \ldots \ldots 126$

B.2. Calculation of Upper Confidence Limits $\ldots \ldots \ldots \ldots \ldots \ldots \ldots \ldots \ldots \ldots \ldots \ldots \ldots \ldots$ 


\section{FIGURES}

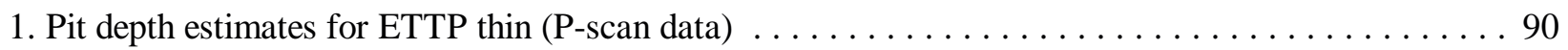

2. Pit depth estimates for ETTP thin, K-yard bottom $\ldots \ldots \ldots \ldots \ldots \ldots \ldots \ldots \ldots \ldots \ldots \ldots \ldots$

3. Pit depth estimates for ETTP thin, except K-yard bottom $\ldots \ldots \ldots \ldots \ldots \ldots \ldots \ldots \ldots \ldots \ldots$

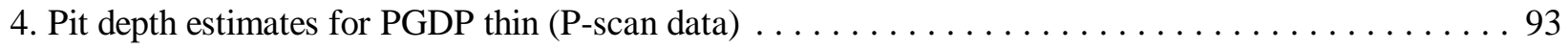

5. Pit depth estimates for PGDP thin bottom, former G-yard $\ldots \ldots \ldots \ldots \ldots \ldots \ldots \ldots \ldots \ldots$

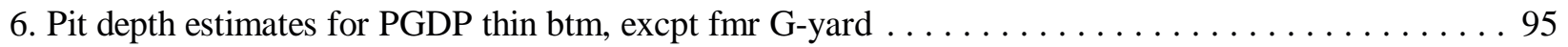

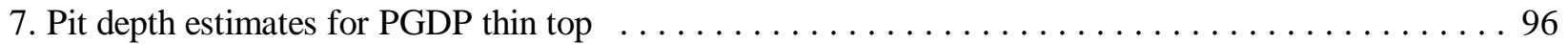

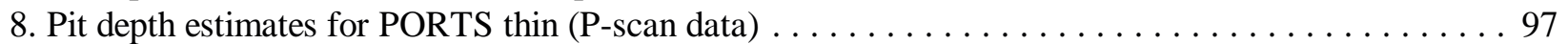

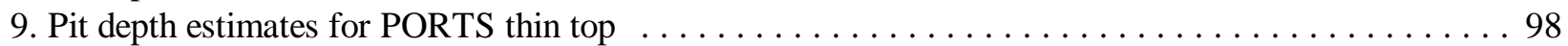

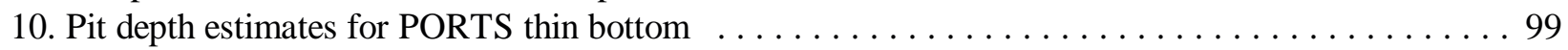

11. Pit depth estimates for ETTP/PGDP/PORTS thick top $\ldots \ldots \ldots \ldots \ldots \ldots \ldots \ldots \ldots \ldots \ldots$

12. Pit depth estimates for ETTP/PGDP/PORTS thick bottom $\ldots \ldots \ldots \ldots \ldots \ldots \ldots \ldots \ldots \ldots 1$

13. Pit depth estimates for ETTP/PGDP/PORTS thin skirted top $\ldots \ldots \ldots \ldots \ldots \ldots \ldots \ldots \ldots \ldots$

14. Pit depth estimates for ETTP/PGDP/PORTS thin skirted $b t m \ldots \ldots \ldots \ldots \ldots \ldots \ldots \ldots \ldots$

15. Pit depth estimates for ETTP/PGDP/PORTS thick skirted $\ldots \ldots \ldots \ldots \ldots \ldots \ldots \ldots \ldots$

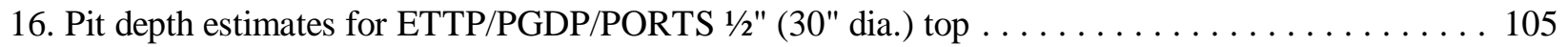

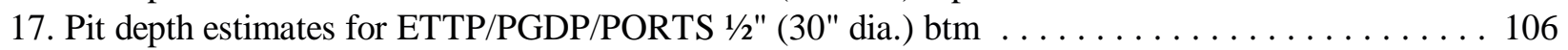

18. Minimum thicknesses (top) and cylinder age distribution (below) for ETTP thin (P-scan data

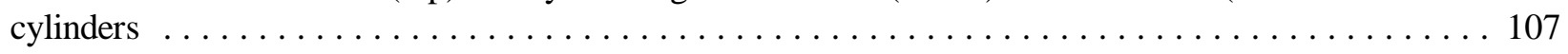

19. Minimum thicknesses (top) and cylinder age distribution (below) for ETTP thin, K-yard bottom

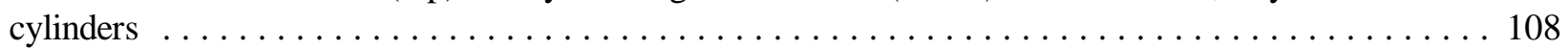
20. Minimum thicknesses (top) and cylinder age distribution (below) for ETTP thin, except K-yard

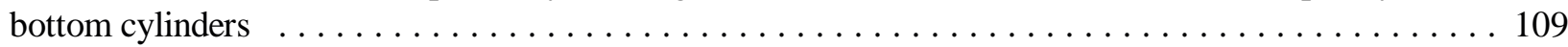

21. Minimum thicknesses (top) and cylinder age distribution (below) for PGDP thin (P-scan data)

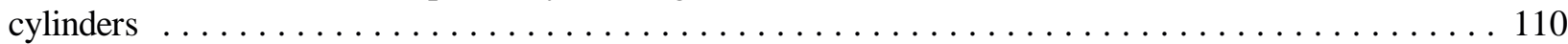
22. Minimum thicknesses (top) and cylinder age distribution (below) for PGDP thin bottom, former

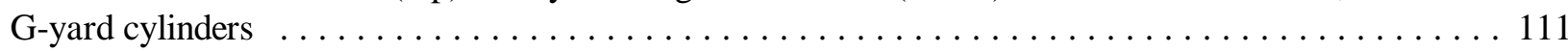
23. Minimum thicknesses (top) and cylinder age distribution (below) for PGDP thin btm, excpt fmr

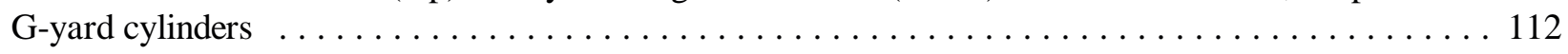
24. Minimum thicknesses (top) and cylinder age distribution (below) for PGDP thin top cylinders . . . 113 25. Minimum thicknesses (top) and cylinder age distribution (below) for PORTS thin (P-scan data

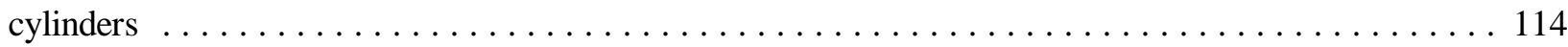

26. Minimum thicknesses (top) and cylinder age distribution (below) for PORTS thin top

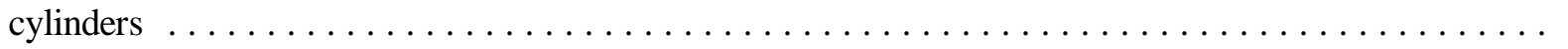
27. Minimum thicknesses (top) and cylinder age distribution (below) for PORTS thin bottom

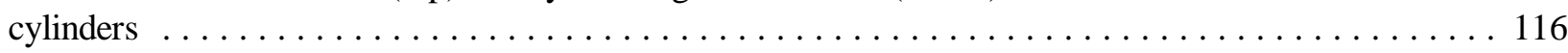
28. Minimum thicknesses (top) and cylinder age distribution (below) for ETTP/PGDP/PORTS

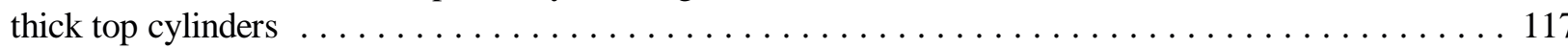

29. Minimum thicknesses (top) and cylinder age distribution (below) for ETTP/PGDP/PORTS

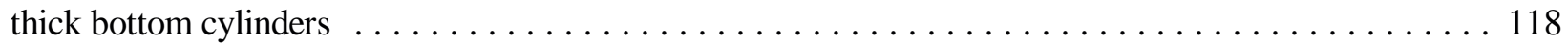
30. Minimum thicknesses (top) and cylinder age distribution (below) for ETTP/PGDP/PORTS thin skirted top cylinders . . . . . . . . . . . . . . . . . . . . . . . . . . . . . . . . . . 119 31. Minimum thicknesses (top) and cylinder age distribution (below) for ETTP/PGDP/PORTS thin

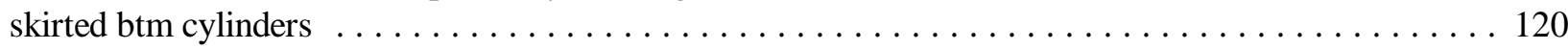
32. Minimum thicknesses (top) and cylinder age distribution (below) for ETTP/PGDP/PORTS thick

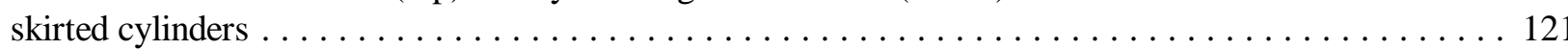
33. Minimum thicknesses (top) and cylinder age distribution (below) for ETTP/PGDP/PORTS $1 / 2 "$

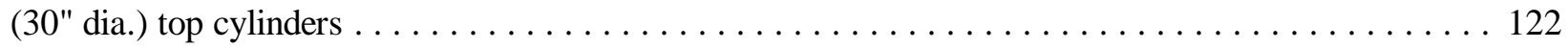
34. Minimum thicknesses (top) and cylinder age distribution (below) for ETTP/PGDP/PORTS 1/2"

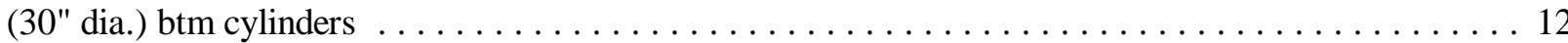




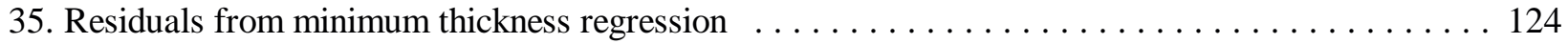

36. Normal probability plot for the regression residuals $\ldots \ldots \ldots \ldots \ldots \ldots \ldots \ldots \ldots \ldots \ldots$

37. Example of a joint confidence line over an interval, based on two joint UCL's computed at

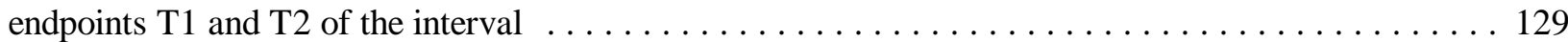




\section{TABLES}

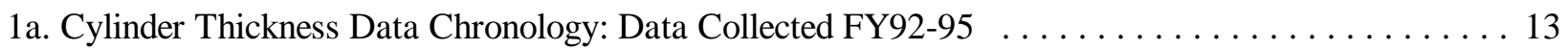

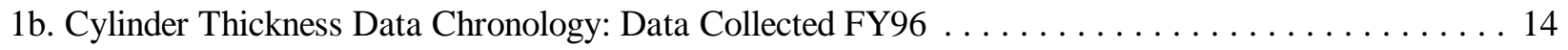

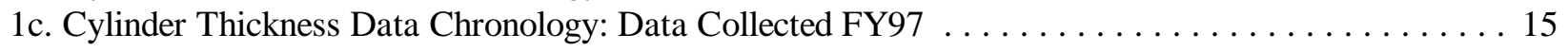

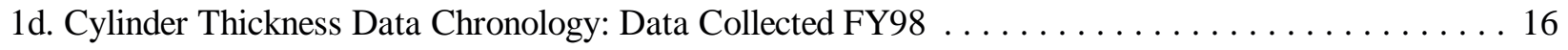

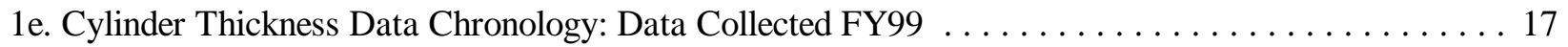

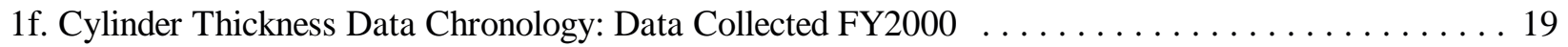

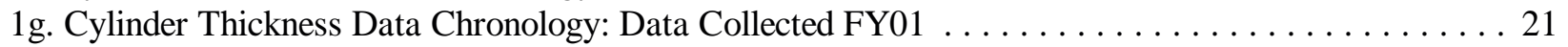

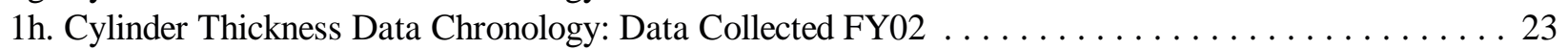

2a. ETTP Cylinder Population and Thickness Data Inventory $\ldots \ldots \ldots \ldots \ldots \ldots \ldots \ldots \ldots \ldots$

2b. PGDP Cylinder Population and Thickness Data Inventory . . . . . . . . . . . . . . . . 29

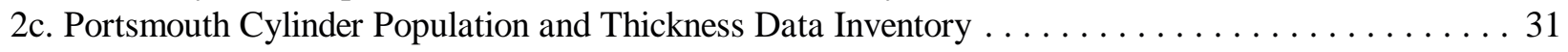

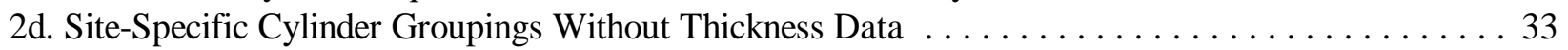

3. The Seventeen Subpopulation Classification System Used in the Regression Modeling . . . . . . 36

4. Summary of Indirect-Model Populations and Modeling Assumptions . . . . . . . . . . . . . 38

5. Comparison of Estimated Minimum Point Wall Thickness Using Different

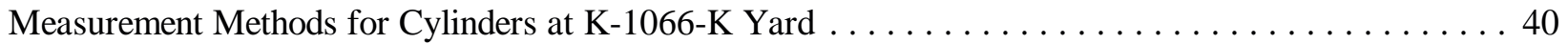

6. 1/2" (30" dia.) Cylinder Minimum Thicknesses and Estimated Pit Depths by

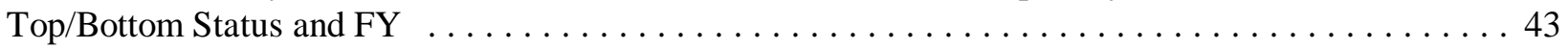

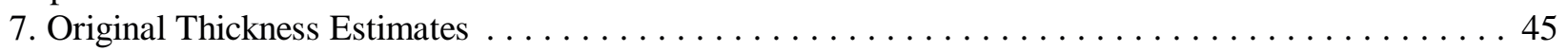

8. Direct-Model Parameter Estimates and Standard Errors $\ldots \ldots \ldots \ldots \ldots \ldots \ldots \ldots \ldots \ldots \ldots 47$

9A. Summary of Indirect-Model Projections for Target Years and Minimum Thickness Criteria . . . . 49

10A. Summary of Direct-Model Projections for Target Years and Thickness Criteria . . . . . . . . 52

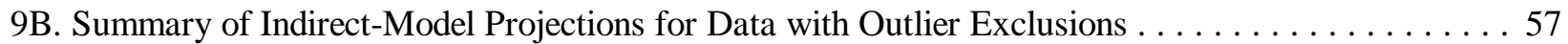

10B. Summary of Direct-Model Projections for Data with Outlier Exclusions . . . . . . . . . . . 60

9C. Indirect-Model Projections (Projected Proportions) for Target Years and

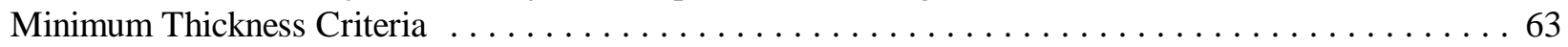

10C. Direct-Model Projections (Projected Proportions) for Target Years and

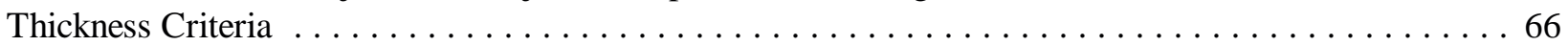

11. Cylinders Excluded from Tables 9B (Indirect Model) or 10B (Direct Model) . . . . . . . . . 69

12. Cylinder Population/Age Groups With Highest Estimated Percentage Failing

the 62.5 Mil Thickness Criteria (By Descending Direct-Model Percentage Estimate) . . . . . . . . . 71

13. FY02 Indirect and Direct-Model Projected and Observed Counts for Sampled Cylinders . . . . . . 79

14. Values of the Log-likelihood and Akaike Information Criterion (AIC) $\ldots \ldots \ldots \ldots \ldots \ldots \ldots$ 


\section{ACRONYMS/ABBREVIATIONS}

AIC

ANSI

BJC

CID

DOE

DUF $_{6}$

ETTP

FY

LCL

mil

ORNL

PGDP

PORTS

SEMP

SRD

$\mathrm{UF}_{6}$

UCL

UT
Akaike Information Criterion

American National Standards Institute

Bechtel Jacobs Company, LLC

Cylinder Inventory Database

Department of Energy

Depleted uranium hexafluoride

East Tennessee Technology Park

Fiscal Year

Lower confidence limit

0.001 inch

Oak Ridge National Laboratory

Paducah Gaseous Diffusion Plant

Portsmouth Gaseous Diffusion Plant

System Engineering Management Plan

System Requirements Document

Uranium hexafluoride

Upper confidence limit

Ultrasonic thickness 


\section{ACKNOWLEDGMENT}

The authors would like to thank Dr. Steven J. Pawel, Corrosion Science \& Technology Group, Oak Ridge National Laboratory, for carefully reviewing this report, and for many helpful suggestions. 


\section{EXECUTIVE SUMMARY}

Depleted uranium hexafluoride $\left(\mathrm{UF}_{6}\right)$ is stored in over 60,000 steel cylinders at the East Tennessee Technology Park (ETTP) in Oak Ridge, Tennessee, at the Paducah Gaseous Diffusion Plant (PGDP) in Paducah, Kentucky, and at the Portsmouth Gaseous Diffusion Plant (PORTS) in Portsmouth, Ohio. The cylinders range in age from six to 52 years. Although when new the cylinders had wall thicknesses specified to within manufacturing tolerances, over the years corrosion has reduced their actual wall thicknesses. The $\mathrm{UF}_{6}$ Cylinder Project is managed by the United States Department of Energy (DOE) to safely maintain the $\mathrm{UF}_{6}$ and the cylinders containing it. The requirements of the Project are delineated in the System Requirements Document (LMES 1997a), and the actions needed to fulfill those requirements are specified in the System Engineering Management Plan (LMES 1997b).

This report documents activities that address requirements and actions involving forecasting cylinder wall thicknesses. Wall thickness forecasts are based on models fit to ultrasonic thickness (UT) measurement data. First, UT data collected during FY02 is combined with UT data collected in earlier years (FY92FY01), and all of the data is inventoried chronologically and by various subpopulations. Next, the data is used to model either maximum pit depth or minimum thickness as a function of cylinder age, subpopulation (e.g., PGDP G-yard, bottom-row cylinders), and initial thickness estimates. The fitted models are then used to extrapolate minimum thickness estimates into the future and to compute estimates of numbers of cylinders expected to fail various thickness criteria. A model evaluation is performed comparing UT measurements made in FY02 with model-fitted projections based only on data collected before FY02.

The FY02 UT data, entered into the corrosion model database and not available for the previous edition of this report (Schmoyer and Lyon 2002), consists of thickness measurements of 48" thin-wall cylinders: 102 cylinders at Paducah, 104 at ETTP, and 117 at Portsmouth; and 72 thick-wall cylinders at Portsmouth. However, because of missing values, repeated measures on the same cylinders, outliers, and other data problems, not all of these measurements are necessarily used in the corrosion analysis, and some previous measurements may simply be replaced with the new ones.

In this edition of the report, cylinder subpopulation definitions and counts are updated using the latest (as of June 2003) information from the Cylinder Inventory Database (CID). Cylinders identified in the CID as painted during the last ten years are excluded from subpopulations considered at-risk of failing minimum thickness criteria, because it is assumed that painting fully arrests corrosion for ten years. As in the previous edition of the report, two different approaches to corrosion modeling are pursued: (1) a direct approach in which minimum thickness is modeled directly as a function of age, subpopulation, and initial thickness estimates; and (2) an indirect approach, in which maximum pit depth is modeled, and the pitdepth model is then combined with a model of initial thickness to compute estimates of minimum thickness.

The data used for both the direct and indirect models is from cylinders sampled randomly or approximately randomly starting in FY92. In the earlier years, P-scanning was the primary UT measurement method. Although the P-scan data is still included in the modeling, it is deprecated in the discussion-for all three sites, not just ETTP and Portsmouth, as in previous editions of the report. With the exception of some Portsmouth cylinders that were scanned several times over the years, the sampling design is crosssectional-each year, new samples of cylinders are selected for scanning.

Both the direct and indirect corrosion models suggest the ETTP thin-wall cylinders, both K-yard bottom and the remainder, are the most likely to fail various thickness specifications. The next most vulnerable cylinder subpopulations are the ETTP/PGPD/PORTS thin, skirted bottom cylinders (at the head/skirt interface) and the PGDP former G-yard, bottom-row cylinders. Very few of the thick-wall or 1/2" (30" diameter) cylinders show any indication of failing any of the thickness criteria. These conclusions pertain to both near-term (e.g., FY03) and longer-term projections (e.g., FY2020). 
In this edition of the report, considerable attention is paid to statistical outliers. Projections of numbers of cylinders likely to fail various thickness criteria are computed for the direct and indirect models, both with and without certain outliers in the analysis. Removing only a small percentage $(<1 \%)$ of the data used to fit the models turns out to have a substantial effect on the fitted models and computed projections. A recommendation of the report is that cylinders with outlier UT measurements should be measured again to either verify or correct earlier UT measurements for them. Because the justification for throwing the outliers out is only statistical, it is important to remeasure these cylinders. (Remeasurements of some of these cylinders are already being made at Paducah.)

In previous editions of the report, model-based projections have been computed for each of various cylinder subpopulations. New in this edition are tables of model projections by both subpopulation and age. Because overall projections for any given subpopulation are averages over all ages, age-specific projections that warrant attention can be identified in the new tables, but may not be identified in projections for the subpopulation as a whole. For example, for the subpopulations of Portsmouth, thin-wall, bottom-row cylinders and ETTP, thin-wall, K-yard, bottom-row cylinders, overall projections of numbers of cylinders likely to fail various thickness criteria are much lower (on the whole) for Portsmouth, but age-specific projections for cylinders of age 47 (for example) are nearly as high for Portsmouth as ETTP. Because the Portsmouth cylinders are not as old on the whole as the ETTP cylinders, this is not clear from the tables of overall projections for cylinder subpopulations, not broken down by age.

The minimum wall thickness criteria used in this report are as follows. For thin-wall cylinders (design nominal wall thickness 312.5 mils; 1 mil $=0.001$ inch): 0 (breach), 62.5, and 250 mils. For thick-wall cylinders (design nominal wall thickness 625 mils): 0, 62.5, and 500 mils. For 1/2" (30" diameter) cylinders: $0,62.5$, and 100 mils. These criteria triples are based respectively on (1) loss of $\mathrm{UF}_{6}$ (breaching), (2) safe handling and stacking operations, and (3) standards for off-site transport and contents transfer criteria.

For the higher thickness criteria (250/500/100 mils for thin-wall, thick-wall, or 1/2" cylinders respectively), FY02 cylinder thickness measurements are consistent with projections based only on measurements made before FY02. For the lower thickness criteria, no failures of the criteria are predicted and none are observed. This finding is somewhat inconclusive, however, simply because failures of cylinders to meet the lower criteria are so rare. Projections for the entire population of cylinders, as opposed to just those sampled, appear, in fact, to be conservative. Failures of the 0 and 62.5 mil criteria are projected at a rate that, though very small, would be unlikely to go undetected, even without UT measurements. Because of updating of the subpopulation definitions, deprecation of the P-scan data, and the addition of new data for measurements made in $\mathrm{FY02}$, these projections are considerably lower than the corresponding projections for FY02 computed for the FY02 report. Nevertheless, the projections still appear to be conservative.

For the direct model, projections can be conservative because of regression parameter estimation error, for which the direct model projections incorporate adjustments. The indirect model projections do not account for parameter estimation error. Another possible reason for the conservative projections is that cylinders were once maintained at less than present-day standard, and that, even after accounting for attenuation in the rate of corrosion, current corrosion is not as severe as corrosion in the past. However, essentially all of the UT measurement data is from FY94 and later. So corrosion changes reflected in the UT data itself (i.e., as differences - no UT initial thickness measurements have been made) are all changes subsequent to FY94. At least in theory then, it should be possible to quantify current cylinder minimum thicknesses or pit depths and current rates of change using available UT data, and the rationale that cylinders were once maintained at less than present-day standards might not be enough to explain conservative projections. 
Other possible reasons for conservative projections include:

- problems with the corrosion physical model (model lack of fit even under constant conditions)

- data anomalies (outliers) and how they are handled in the statistical analysis.

As discussed above, data anomalies are a focus of this report. Problems with the corrosion physical model were considered in Schmoyer and Lyon (2002), where the direct modeling approach is proposed as an alternative to the indirect modeling approach used in previous editions of the report. Because of problems such as the difficulty in measuring original thicknesses, the indirect model has lead to anomalous results, sometimes suggesting accelerated or even "negative" corrosion, which are inconsistent with corrosion theory and practical experience. Therefore, the alternative, direct model was developed in the last edition and is considered again here. The direct approach seems better than the indirect approach in several ways. It seems to have better statistical properties, and it does not lead to either accelerated or "negative" corrosion fits. However, like the indirect-model projections, the direct-model projections also seem conservative. Thus, as before, the conclusion of this report is that neither modeling approach is clearly better or worse than the other and both approaches should continue to be explored.

In addition to age and subpopulation, a myriad of other variables might effect corrosion but are not accounted for in the modeling. Examples include how many use cycles the cylinders went through, how many nicks and scrapes, and the nature of former surface coatings, now perhaps long gone. Add to the variables not accounted for, biases introduced in the UT measurement scanning, and the result is a problem with lots of statistical error. Corrosion physics and the effects of time are only a part of the whole story. In addition to a good model, good cylinder thickness forecasts are and will continue to be contingent on careful UT measurements, careful data quality control (e.g., elimination of outliers), and sufficient UT data that, through laws of large numbers, the statistical noise will be reduced.

This report complements and extends previous editions by Lyon (1995, 1996, 1997, 1998, 2000) and by Schmoyer and Lyon (2001, 2002). 


\section{INTRODUCTION}

The $\mathrm{UF}_{6}$ Cylinder Project, managed by the United States Department of Energy (DOE), was formed to maintain and safely manage depleted uranium hexafluoride $\left(\mathrm{UF}_{6}\right)$ stored in over 60,000 carbon steel cylinders. The cylinders are located at three DOE sites: the East Tennessee Technology Park (ETTP) in Oak Ridge, Tennessee; the Paducah Gaseous Diffusion Plant (PGDP) in Paducah, Kentucky, and the Portsmouth Gaseous Diffusion Plant (PORTS) in Portsmouth, Ohio. The results presented here complement and extend those presented in previous editions of cylinder corrosion reports by Lyon (1995, 1996, 1997, 1998, 2000), and Schmoyer and Lyon (2001, 2002).

The $U F_{6}$ Cylinder Project System Requirements Document (SRD, LMES 1997a) delineates the requirements of the project, and the actions needed to fulfill those requirements are specified in the $U F_{6}$ Cylinder Project System Engineering Management Plan (SEMP, LMES 1997b). This cylinder corrosion report documents activities that address specific requirements and actions stated in the SRD and SEMP with respect to forecasting cylinder wall thicknesses:

System Requirement 1.2.2 is that performance shall be monitored and evaluated to identify potential risks. The corresponding SEMP Action 2.1.2 is to model corrosion to project cylinder integrity. This report establishes techniques for modeling corrosion rates used in the project to forecast cylinder integrity, quantified in terms of wall thickness.

System Requirement 4.1.2 calls for monitoring cylinder conditions. The corresponding SEMP Action 3.1.2 is to statistically determine the baseline condition of cylinder populations by obtaining quantitative data. This report documents statistical methods used to characterize cylinder populations on the basis of UT measurement data. Wall thickness and corrosion pit depth data have been collected for several subpopulations of cylinders.

System Requirement 4.2.1 states that cylinders shall be categorized to ensure that risks are identified. The corresponding SEMP Action 2.2 is to define and describe categories in terms of cylinder functional criteria and/or factors that could adversely impact cylinder integrity. The analyses in this report are based on cylinder populations defined in terms of cylinder types (e.g., thick-wall, thin-wall), historical storage locations (yard and position), and similarity of quantitative data.

System Requirement 4.2.2 states that cylinder conditions shall be forecast to direct surveillance and maintenance resources. The corresponding SEMP Action 2.4 is to define procedures for forecasting cylinder conditions. System Requirement 4.2.2a is to identify which collected data will be used for forecasting (SEMP Action 2.2.1) and to integrate forecasting with modeling efforts (SEMP Action 2.3). System Requirement 4.2.2.b is to develop mechanisms to consolidate information for summary-level decision making, and the corresponding SEMP Action 2.3.1 is to forecast cylinder conditions using the parameters identified. Cylinder wall thickness, the subject of this report, is one parameter identified in the project for forecasting cylinder conditions. SEMP Action 3.1.1 is to project numbers of non-compliant cylinders.

Section 2 of this report introduces two general approaches to modeling and forecasting cylinder wall thicknesses, a "direct" approach and an "indirect" approach based on separate models of pit depth and original thickness. Section 3 is a history and summary of cylinder wall thickness data collection at Oak Ridge, Paducah, and Portsmouth. Section 4 is about regression model fitting with the models introduced in Section 2 and the data discussed in Section 3. As prescribed in SEMP Action 3.1.1, Section 5 contains projections based on the models fit in Section 4. Separate projections are presented for both the direct and indirect modeling approaches. The direct and indirect models are evaluated and compared in Section 6. Limitations, conclusions, and recommendations are discussed in Section 7. 
The disposition of any particular cylinder for storage, handling, and transfer should depend on the condition of the cylinder. In this report "condition" will be the minimum wall thickness of the cylinder. Wall thickness criteria 0, 62.5, and 250 mils for thin-wall cylinders, and 0, 62.5, and 500 mills for thick-wall cylinders, are limits based on (1) loss of $\mathrm{UF}_{6},(2)$ safe handling and stacking operations, and (3) standard off-site transport and contents transfer. In general, these criteria refer to an area of wall thinning, not a single a point. On the other hand, minimum wall thickness measurements collected for the report are for areas of only about 0.01 square inches, which is essentially a point. For thickness criteria greater than zero, conclusions based on minimum wall thicknesses are, in this respect, conservative. Because of the interaction of $\mathrm{UF}_{6}$ with atmospheric moisture and steel, a point breach would deteriorate in a year to oneinch diameter hole (DNFSB 1995), however, and so small-area approximations should be close, at least for the breach criteria.

Projections of numbers of cylinders expected to fail various thickness criteria are the primary objective of this report. For these projections, recently painted cylinders are excluded from populations considered to be at risk. For higher thickness criteria (250/500/100 mils for thin-wall, thick-wall, or $1 / 2$ " cylinders respectively), projections computed only from pre-FY02 data are consistent with measurements made in FY02. However, projections of numbers of cylinders falling below lower thickness criteria ( 0 and 62.5 mils) appear to be conservative. For the direct model, the conservative projections could be due to regression parameter estimation error, for which the direct model projections incorporate adjustments. The indirect model projections do not account for parameter estimation error. Another possible reason for the conservative projections is that cylinders were once maintained at less than present-day standards, and that, even after accounting for attenuation in the rate of corrosion, current corrosion is not as severe as corrosion in the past. However, essentially all of the UT measurement data is from FY94 and later. So corrosion changes reflected in the UT data itself (i.e., as differences-no UT initial thickness measurements have been made) are all changes subsequent to FY94. Therefore the rationale that cylinders were once maintained at less than present-day standards might not be enough to explain the conservative projections. Other possible reasons include ${ }^{1}$

1. problems with the corrosion physical model (model lack of fit even under constant conditions) 2. data anomalies and how they are handled in the statistical analysis

Problems with the corrosion physical model are considered in Schmoyer and Lyon (2002), where a model is proposed that is an alternative to the corrosion model used in previous corrosion reports. For editions of this report before 2002, minimum cylinder thickness had been modeled indirectly as a difference between original thickness and maximum pit depth. Maximum pit depth was modeled as a function of cylinder age. A separate original thickness measurement/estimate was required for each cylinder used to fit the model. Because of the difficulty in measuring original thicknesses for each cylinder and perhaps other problems, the indirect approach has lead to anomalous results, sometimes suggesting accelerating (or even negative) corrosion, which is inconsistent with corrosion theory. Therefore, for the 2002 report, an alternative, "direct" model was considered, in which minimum thickness is modeled as a function of age and an initial thickness estimate for each cylinder population, as opposed to each individual cylinder. The direct approach improved the corrosion predictions in that anomalous model fits seemed to be avoided. However, like the indirect-model projections, projections based on the direct model seemed too conservative. The direct and indirect modeling approaches are both considered again in this report.

\footnotetext{
${ }^{1}$ The sometimes conflicting goals of inspection and characterization can also lead to pessimistically sampled cylinder thickness data and in turn to conservative conclusions. However, that bias is not quantified in this report.
} 
In this edition of the report, considerable attention is given to reason \#2 above. Statistical analyses are performed both with and without certain statistical outliers-less than $1 \%$ of data points-which are most extreme and, in that statistical sense, most suspect. A conclusion from these comparisons is that much of the conservatism in the thickness predictions hinges on whether or not fewer than $1 \%$ of thickness measurements are excluded from the analysis. Furthermore, analyses of cylinders measured during several different years have shown that inconsistencies do occur among repeated measurements (see, for example, discussion about $1 / 2$ " thick (30" dia.) cylinders in Section 3.2). Thus there is a basis for skepticism about some of the more extreme thickness data. A conclusion of this report is that cylinders with anomalous thickness measurements should be remeasured to either confirm or refute their prior data. Remeasurements of cylinders with anomalous thickness measurements are already being made at Paducah. 


\section{APPROACHES TO MODELING CYLINDER WALL THICKNESS}

\subsection{Direct and Indirect Models}

The basic problem addressed in this report is to predict how many cylinders, in cylinder groups (subpopulations) defined by age, location, storage position, etc., will have minimum thicknesses below a specified thickness $z$ by at specified time $t$. For a cylinder randomly selected from such a group, let $M(t)$ denote the minimum wall thickness at time $t . M(t)$ is random because of variations in initial thickness (manufacturing variability), the steel substrate, the corrosion process, and storage conditions.

Consider $\operatorname{Prob}(M(t)<z)$, the probability that $M(t)$ is less than $z$. For a group of $N$ cylinders, the expected number of cylinders with minimum thickness below $z$ at time $t$ is $N \times \operatorname{Prob}(M(t)<z)$. Because the number of cylinders in a group at risk is affected by maintenance (e.g., painting may remove cylinders from a group), $N$ may change over time. For a group of cylinders having various ages, the expected number of cylinders with a minimum thickness below a given value $z$ at time $t$ can be estimated using the relation:

( Expected number of cylinders with minimum thickness below $\mathrm{z}$ at time $\mathrm{t})=$

$$
\sum_{\mathrm{a}}(\text { Number of cylinders of age a at time } \mathrm{t}) \times \operatorname{Prob}(M(t)<z \text {; for cylinders of age a }),
$$

where the summation extends over all cylinder ages a. Note that, because of sampling, the age of (2.1) is an expected number of cylinders. Estimates can also be of actual number of cylinders as opposed to expected numbers. The estimates themselves are usually the same, but confidence limits are ordinarily wider for estimates of actual numbers.

One approach to the problem of estimating (2.1) is to make UT measurements of cylinder wall thicknesses, deliberately trying to locate the actual thickness minima. By doing this for cylinders of various ages and from various subpopulations, data so collected can be used to model the minimum thicknesses as a function of age, subpopulation, and estimates of initial thicknesses. Initial thickness estimates are based on nominal thickness data (from design sheet data), as well as maximum wall thickness measurements, and judgment. In this report, this approach will be called "direct," because minium thicknesses are modeled directly, and because the objective is to make projections about minimum thicknesses. Approximations are incurred in the direct approach because of error in initial thickness estimates, and because actual minimum thicknesses may not be discovered, either because of insufficient searching, or because searches may be focused on areas of maximum pitting rather than the minimum thickness. If a cylinder's initial thickness is not uniform (e.g., because of variations introduced in forming), then where pitting is worst may not be where the minimum thickness actually occurs.

Another approach is to model maximum pit (i.e., corrosion) depths. Pit depth models are much more common in the literature than minimum thickness models (see below). Given a pit depth model, projections about minimum thicknesses can be computed as differences between initial thickness estimates and maximum pit depth estimates computed from the pit depth model. As in the direct approach, initial thickness estimates can be based on nominal specifications and maximum thickness measurements.

Besides the initial thickness approximation, an approach based on maximum pit depths is approximate because minimum thicknesses need not occur where initial thicknesses are minimum or where pit depths are maximum. More specifically, let $C_{0}(x)$ denote the initial wall thickness at a cylinder location $x$, and let $P(t, x)$ denote the pit depth at location $x$ at time $t$. Then the thickness at point $x$ is $C_{0}(x)-P(t, x)$, and the minimum thickness is 


$$
M(t)=\min _{x}\left\{C_{0}(x)-P(t, x)\right\},
$$

where the min is over all points $x$ on the cylinder. For time $t$, let $x^{*}(t)$ denote the point $x$ at which the thickness is minimized. Then $M(t)=C_{0}\left(x^{*}(t)\right)-P\left(t, x^{*}(t)\right)$. Note that $P\left(t, x^{*}(t)\right)$ is the pit depth at the point of minimum thickness, which is not necessarily the maximum pit depth. Similarly $C_{0}\left(x^{*}(t)\right)$ is not necessarily the minimum initial thickness.

If $x^{*}(t)$ is estimated through UT scanning, and if UT thickness measurements are made at $x^{*}(t)$ (approximately) and at relatively uncorroded areas in the vicinity of $x^{*}(t)$, then $P\left(t, x^{*}(t)\right)$ and $C_{0}\left(x^{*}(t)\right)$ can be estimated. Those estimates could be used to develop models for pit depths and initial thicknesses at $x^{*}(t)$, which in turn can be combined to produce minimum thickness estimates and projections. Note, however that this approach would not really be based on a maximum pit depths. Furthermore, the approach would be very heavily dependent on proper thickness measurements being made at relatively uncorroded areas near $x^{*}(t)$. Uncorroded areas might not exist. A very common notation on cylinder reports is "uniform corrosion," which suggests that for those cylinders there are no uncorroded areas.

Lyon (2000) developed a method based on a maximum pit depth model and the following approximation. Observe that

$$
\begin{aligned}
M(t) & \geq \min _{x}\left\{C_{0}(x)\right\}-\max _{x}\{P(t, x)\} \\
& =C_{0}-\max _{x}\{P(t, x)\} \\
& =C_{0}-P(t)
\end{aligned}
$$

where $C_{0}$ is the initial minimum thickness, and $P(t)$ is the maximum pit depth at age $t$. By this inequality, $C_{0}-P(t)$ is a lower bound for $M(t)$, and conclusions about $C_{0}-P(t)$ are conservative conclusions about $M(t)$.

Because it starts with a conservative approximation, Lyon's approach may be more appropriate than the above approach based on estimates of $P\left(t, x^{*}(t)\right)$ and $C_{0}\left(x^{*}(t)\right)$, especially in view of limitations in the UT measurement data. In Lyon's approach, the $C_{0}$ are estimated either with thickness measurements made at uncorroded areas near the area of minimum wall thickness, or else with "original thickness estimates" measured at areas of approximate maximum thickness. The $P(t)$ are estimated by subtracting thickness measurements made either where the worst pitting occurs or where the wall thickness is minimum. (In practice, $x^{*}(t)$ has been estimated by searching with UT scans, but those searches have almost surely been biased towards areas of maximum pitting.) To make estimates and projections about minimum thicknesses, the statistical distributions of the $C_{0}$ and $P(t)$ are combined (see Appendix A) in a way that assumes the two distributions are statistically independent. The statistical independence is an assumption that could fail, for example, if steel quality and initial thickness are correlated. In this report, because the minimum thicknesses are modeled indirectly through separate models of maximum pit depth and initial thickness, Lyon's approach is referred to as "indirect." 


\subsection{The Indirect Approach}

Maximum Pit Depth Models. If the corrosion process has reached a condition in which, whatever the corrosion history may have been, each cylinder is corroding at some relatively constant rate, then modeling future corrosion entails determining the current conditions for each cylinder and estimating the current rate. A feature expected of corrosion, however, is that, in general, its rate decreases with time.

The indirect model used in this report is based on the power law, which has been used in many previous applications of corrosion modeling (e.g., Felieu et al. 1993a; Felieu et al. 1993b; Legault and Preban 1975; Pourbaix 1982; Mughabghab and Sullivan 1989; Romanoff 1957). The power law is $P($ age $)=A \times(\text { age })^{n}$, where $P$ denotes pit depth (or penetration), and $A$ and $n$ are constants. For $n<1$ the power law allows for "leveling off" in corrosion, which is common because of the semi-protective qualities of iron oxides. The model parameters $A$ and $n$ can be estimated using the log-linear regression model

$$
\log (P(\text { age }))=\log (A)+n \log (\text { age })+\text { random error, }
$$

which is the estimation approach taken in this report. (All logs in this report are natural logs.) The random errors are assumed to be statistically independent and independent of the cylinder initial thickness.

Separate regression models are fit for each of seventeen cylinder groupings, which are discussed in Sections 3 and 4. For the regressions, maximum pit depth measurements for each cylinder are estimated from minimum thickness measurements and estimates of initial thickness, which are based on maximum wall thickness measurements made for each cylinder.

According to Pourbaix (1982), Passano (1934) was the first to use the power law relationship in corrosion prediction. This law is considered to be valid for different types of atmospheres (rural, marine, industrial) and a number of materials. The parameter $A$ can be interpreted as the corrosion in the first year, and the parameter $n$ represents the attenuation of the corrosion because of the passivation of the material in the atmosphere (Pourbaix, p.115).

The power law model can be related to the mean (age-averaged) corrosion rate, since the mean corrosion rate is given by $P /$ age $=A \times(\text { age })^{n-1}$. If $n=1$, this implies that the age-averaged corrosion rate is constant, while if $n<1$ (which is typical), the corrosion rate decreases with time. Mechanistic interpretations of $n$ have also been made (Horton 1964). If $n=0.5$, then the relationship is said to be parabolic, with the corrosion rate controlled by diffusion through the rust layer. If $n<0.5$, the rust layer is showing protective properties, while if $n>0.5$, the rust layer is not fully protective because of factors that may be preventing the homogeneous thickening of the rust layer.

Because estimates of the "leveling off" $(n<1)$ pattern usually expected for pit depths can be sensitive to narrow data ranges, outliers, and other data anomalies, the power law approach should be used with caution. In fact, a failure of either the leveling off $(n<1)$ hypothesis or the increasing corrosion $(n>0)$ hypotheses is observed for eight of the seventeen cylinder groups considered in this report (see Section 4), and an alternative model is then applied. The alternative model is the same, except that $n$ is constrained to be 1 (Lyon 1995, 1996). ${ }^{2}$ (This inconsistency between data and assumption- the need for the slope-set-toone-model—was the main motivation for the direct approach.)

In order to address the variability inherent in the corrosion process, it will be assumed in the indirect model that pit depths are lognormally distributed at each age (or time). This can also be expressed on the log

\footnotetext{
${ }^{2}$ Because, $n<0$ is untenable from both a theoretical and practical perspective, the same $n=1$ (slope-setto-one) alternative will be used if the power-law model estimate of $n$ is either greater than 1 or less than 0 .
} 
scale as $\log (P(a)) \sim N(\log (A)+n \log (a), \sigma)$, where $N(\mu, \sigma)$ denotes a normal distribution with mean $\mu$ and standard deviation $\sigma$. For this model, on the arithmetic scale, the median is equal to $A(\text { age })^{n}$, the mean is $A(\text { age })^{n} \exp \left[\sigma^{2} / 2\right]$, and the standard deviation is $A(\operatorname{age})^{n} \exp \left[\sigma^{2} / 2\right]\left[\exp \left(\sigma^{2}\right)-1\right]^{1 / 2}$. The coefficient of variation (ratio of the standard deviation to the mean) is constant in time and equal to $\left[\exp \left(\sigma^{2}\right)-1\right]^{1 / 2}$.

The lognormal assumption has been checked by goodness of fit tests discussed in previous cylinder reports (Lyon 2000). Given that the data consists of maximum pit depth estimates, it would be natural to apply extreme-value statistics to this problem. Application of the extreme value distribution (without confidence limits) is discussed in several papers and has also been suggested within the Project by Rosen and Glaser (1996). The basic idea is that for $\mathrm{P}$, the maximum pit depth in $\mathrm{m}$ pit depth measurements made on a randomly selected cylinder, there is a standardization $\mathrm{a}_{\mathrm{m}}$ and $\mathrm{b}_{\mathrm{m}}$ (depending only on $\mathrm{m}$ ) such that as $\mathrm{m}$ increases, the statistical distribution of $a_{m}+b_{m} \times P$ converges to a particular parametric form known as the extreme value distribution (see, for example, David 1981). Although extreme value theory should be investigated, as yet unresolved statistical issues about data quality, outliers, the corrosion-age relationship, and the choice of the basic corrosion model (e.g., indirect vs direct) need to be resolved before investigating whether or how to apply extreme value theory. Note that the direct model (see below) is nonparametric (not based on any parametric distribution) and does not require choosing any form of parametric statistical distribution.

Initial Thickness Models. A stochastic model is used for initial thicknesses, in the indirect modeling approach, because of concerns that variability in initial thickness could be a critical factor (Rosen and Glaser 1995). (In the direct approach, variability in initial thickness is modeled as part of the variability of the minimum wall thicknesses.) With the exception of cylinders purchased very recently, there is no way to know the distributions of initial thicknesses. Therefore the initial thickness distribution is approximated with a truncated normal distribution (see Johnson and Kotz, 1970, p 81.) A truncated normal random variable has the distribution of a normal random variable conditional on the normal variable being in the truncated range. The lower end of the truncation range is taken as the lower end of the design ranges, $312.5,615$ mils respectively for thin and thick-wall cylinders, and, for $1 / 2$ " diameter cylinders, as 490 mils, that is, ten mils less than the $1 / 2$ " nominal thickness. The upper end of the truncation range, as well as the mean and standard deviation of the underlying normal distribution are determined from maximum wall thickness data.

With the exception of the data at the head/skirt interface (discussed below), the maximum wall thickness data consists of wall thickness measurements made on the cylinders measured in relatively uncorroded regions of the cylinder. The data collected so far suggest that the maximum wall thickness at relatively uncorroded cylinder areas is usually larger than the nominal design thickness. Note, however, that if there is uniform corrosion, then maximum thickness measurements could underestimate the design maximum thickness.

Probability and Confidence Limits. Estimates of probabilities that cylinders will fail various thickness criteria can be calculated using a convolution of the pit depth and initial-thickness statistical distribution estimates, which are assumed to be (statistically) independent. Confidence limits can be computed using the same convolution along with confidence bounds for the regression parameter estimates. These calculations are derived in Appendix B.

\subsection{The Direct Approach}

Direct models were investigated as an alternative to the indirect approach, because of anomalous results based on the indirect approach, due in part to the high variability that has been seen in the minimum thickness and maximum pit depth data. Thickness projections based on the direct model were first computed in the 2002 edition of this report (Schmoyer and Lyon 2002); indirect model projections were computed in the 2002 edition as well as previous editions. Although the direct model appears to perform 
favorably, a conclusion of the 2002 report is that both models should continue to be explored. Modeling results for both the direct and indirect approaches are discussed in Section 4.

There are other reasons, besides just data variability, for exploring alternatives to the indirect approach. A pre-existing pit holds moisture differently than a uniform surface, for example, and because of the thermal inertia of the cylinders, literature data for atmospheric corrosion of steel does not necessarily apply to cylinder corrosion modeling. ${ }^{3}$ Thus, the power law may not apply.

The direct model considered in this report is

$$
M(\text { age })=\alpha \times(\text { Initial Thickness Estimate })+\beta(\text { group }) \times \log (\text { age })+\text { random error, for age }>1,
$$

where $\alpha$ is a model parameter, and " $\beta$ (group)" denotes a model parameter, one for each cylinder group. The " age $>1$ " condition is imposed, because $\log ($ age $)$ is unbounded and negative for age $<1$, and so the model cannot hold for arbitrarily small ages. Presumably another model would hold, but the point is moot as far as this report is concerned, because there are no thickness measurements for ages less than one year.

According to this model, $M(1)=\alpha \times$ (Initial Thickness Estimate) is the mean thickness at age one year, which is essentially, though not exactly, the initial thickness. Thus the expression "initial thickness estimate" is used loosely here. Initial thickness estimates, which are computed from design specifications, maximum thickness measurements, and judgment, are incorporated into the model as predictors, but are also further refined by fitting the parameter $\alpha$-using minimum thickness measurements. Thus, the direct model is designed to best fit current minimum thicknesses, and the estimate of $a$ is adjusted to improve the current fit. The indirect model best fits current pit depth. If past storage and handling practices have improved, then these pit depths projected into the future will tend to be conservative. Because the $\alpha$ parameter estimate is adjusted to fit the data, the direct model's dependence on initial thickness is less critical. Previous corrosion trends should not have such a conservative effect on the direct model projections, as long as thickness data is collected when maintenance and storage activities are performed at present-day standards.

The cylinder groups for the direct model are the same as for the indirect model (except that thin-wall cylinders are not included in the thick-wall groups; see Sections 3 and 4). However, unlike the indirect approach, in which a separate model (with its own intercept and slope) is fit for each group, in the direct approach considered here, there is one model with separate parameters for each cylinder group. For the indirect model, the total number of parameters (including standard deviations) is three times the number of groups, not including additional estimated parameters in the initial thickness distributions. For the direct model, the total number of parameters is the number of groups plus two (including one for the standard deviation).

Having fewer parameters can be either a disadvantage or an advantage. Models with fewer parameters are less flexible, but if they fit, less flexibility reduces the likelihood that anomalous data will lead to anomalous modeling results, which is a difficulty in the indirect approach.

For either the direct or indirect approaches, how random error terms and their variances are modeled can have a critical effect on corrosion projections. Regardless of the mean minimum thickness, if the variance of the (true) error term is high enough, there will always be cylinders whose minimum thicknesses are below any of the various thickness criteria. (This applies to both the direct and indirect models.) Because, in the direct approach, multiple cylinder groups are handled with one model, high variability in one group affects projections for all groups. Therefore several ways of relating variance to age and consequential

${ }^{3}$ Steve Pawel, ORNL Corrosion Science \& Technology Group, personal communication. 
regressions weightings were considered for the direct model (see Section 4). Further, the random error term in the direct model is not assumed to be lognormal or, in fact, to have any particular distribution. A nonparametric method is used instead.

The direct model avoids pit depth estimation, which, because of limitations in maximum thickness estimates, the UT data does not seem to support well. In addition to data variability and thermal inertia, this may be because the power law model assumes that pits are due to corrosion, not nicks and cuts and handling wear typical of many of the $\mathrm{UF}_{6}$ cylinders, or the interaction between nicks and cuts and corrosion (e.g., through galvanic effects). Another reason may be that both $M$ (age) and $P($ age) are extremes (i.e., the minimum thickness and the maximum pit depth). The rate of change of an extreme (e.g., maximum pit depth) might not satisfy the same relationship to age or time as the rate for an individual element, because which particular element is an extreme can change.

In the direct model and in the indirect model with $0<n<1$, corrosion is assumed to be a concave increasing function of age. In the indirect model, the corrosion rate is $d P($ age $) / d($ age $)=n P($ age $) /($ age $)$ (with a different parameter $n$ for each cylinder group). Thus the corrosion rate depends on the pit depth. In the direct model, the corrosion rate is $-d M($ age $) / d($ age $)=\beta($ group $) /$ age, which decreases with age, but does not depend explicitly on pit depth.

Direct-model estimates of probabilities of failing various thickness criteria are based on a nonparametric analog of usual normal-theory regression prediction bounds for individual predicted values (Schmoyer 1992). A lower prediction bound $L$ (whether parametric or nonparametric) satisfies $\operatorname{Prob}(Y>\mathrm{L})=\gamma$ for some specified confidence level $\gamma$. Given a method for computing $L$ for a specified $\gamma$, a probability $\gamma$ can be estimated for any specified $L$, such as $0,62.5$, or 250 mils. Probability estimates computed this way are the endpoint of the direct model approach. A limitation of this approach is that because the nonparametric confidence bounds are not premised on a continuous distribution, they are not appropriate for values of $\gamma$ less than $1 /(2(n+1))$, where $n$ is the number of observations in the regression used to compute them. Further details are in Section 4.

For these reasons, and because it seems to fit, the direct model was considered as a possible alternative to the indirect approach. 


\section{ULTRASONIC THICKNESS DATA}

This section summarizes the ultrasonic thickness (UT) measurement data used in the corrosion models. The previous version of this report (Schmoyer and Lyon 2002) was based on wall thickness data that had been collected through FY01. This report incorporates additional data collected in FY02. The data collection is chronicled by fiscal year in Section 3.1, and in Section 3.2, by subpopulations defined by cylinder type (thin-wall, thick-wall, $1 / 2 ")$, whether the data is for the head/skirt interface or the main cylinder body, top/bottom storage status, etc. Cylinders are often moved during their lifetimes, but only one location is used to represent each cylinder for the purpose of grouping cylinders for modeling. Complete historical records are usually not available in an electronic form, and even if they were, using such records to incorporate multiple-location histories into the models would be very complicated.

Two main types of data are used in the corrosion modeling:

(1) data for predicting overall minimum wall thickness at points not including the head/skirt interface

(2) data for predicting minimum wall thickness at the head/skirt interface

In most cases both minimum and maximum wall thicknesses estimates were measured. The minimum thickness measurements are plotted in Figures 18-34 in Appendix A.

In past editions of this report, the data from two breached cylinders discovered by visual inspection in FY92 in K-1066-K yard was used in the corrosion modeling. External corrosion was considered to be the cause of these breaches. There have been several other breaches discovered (two at ETTP in FY92, two at Portsmouth in FY90, and one at PGDP in FY92), but it was concluded that those breaches were induced by mechanical damage at the time of stacking rather than by external corrosion, ${ }^{4}$ and so thickness data for those other breached cylinders has not been used in the modeling.

A reason for possibly excluding the breach data for even the corrosion-induced breaches from the corrosion analyses is that those breaches were discovered by visual inspection rather than random sampling. Therefore, including them would induce a pessimistic bias. A reviewer of the 2002 report expressed another reason for excluding them - that the breaches "represent a corrosion situation that simply no longer exists (e.g., corr rate $\approx 30$ mils/yr), and using data skewed by that to project future conditions is technically inappropriate." ${ }^{5}$ Of course this argument could also be made for other cylinders besides those that have breached. Much of the cylinder thickness data used for this report is for cylinders that have had at least a part of their lifetimes when conditions may have been similar to the corrosion situation for the breaches. Nevertheless, the reviewer's comment is addressed in this report as follows: In both the direct and indirect approaches, pre-FY98 thin-wall cylinder data is modeled separately. This is the P-scan data plus the two breaches. Although the P-scan data and the breach data are included in the analyses, that data is deprecated in the interpretation, and the breaches have little influence on the final conclusions.

For this report, hand-held UT methods (Lykins and Pawel 1997) were used for all wall thickness data collected in FY98 and later. Except for head/skirt interface data and for the data for the two breached cylinders, UT measurements from before FY98 were made with an automated P-scan system (see Schmidt et al 1996 for a description of the equipment). P-scan measurements were made during FY94 at K-1066-K yard at ETTP, in the fall of 1995 at PGDP, between March and September 1996 at both Portsmouth and

\footnotetext{
${ }^{4}$ These breaches were caused by a lifting lug of an adjacent cylinder that induced a small crack near a stiffening ring (Barber et al, 1994).

${ }^{5}$ Steve Pawel, ORNL Corrosion Science \& Technology Group, personal communication..
} 
PGDP (as part of cylinder relocation efforts), and during FY97 primarily at Portsmouth. The P-scan wall thickness data consists of measurements made for a square region of width and height about $2.54 \mathrm{~mm}$ (0.1 in). Wall thickness data used to estimate the initial thickness consisted of either P-scan data collected near where the maximum-depth pit occurred (with a width and height of approximately the same size as the for the pit data), or was collected using a hand-held probe for a circular region with a radius of about $2 \mathrm{~mm}$ (0.08 in).

\subsection{Data Collection by Fiscal Year}

In this section data collections are discussed in order of the fiscal year they were performed. Tables 1a-h are inventories of the data organized this way.

FY92. (See Table 1a.) This data consists of the two breached cylinders discovered in FY92 in K-1066-K yard, for which it was deemed that external corrosion was the cause of the breach. The breached cylinders are classified with other pre-FY98 (P-scan) data in the regression analyses performed with this data and are also excluded as outliers in several of the analyses (Section 4).

FY94. (See Table 1a.) Between December 1993 and May 1994, wall thickness measurements were made for 136 cylinders in K-1066-K yard (Philpot 1995) using an automated scanner. It was intended that the cylinders selected for measurement should be chosen at random, though a random number generator was not used to select them, and there were limitations imposed by the automated scanner (e.g., length of power cord, clearance between adjacent cylinders). For these reasons, the cylinders selected are not a truly random sample from the population, though they may emulate a random sample. For the first 21 cylinders evaluated, only minimum wall thickness data was recorded, while maximum thicknesses were also recorded for the remaining 115 cylinders. There were also questions about the accuracy of the wall thickness data for the first group of cylinders. Further, since maximum thickness data was not recorded for the first 21 cylinders, maximum pit depths could not be used for these cylinders, and they are not included in either the direct or indirect-model analyses in this report. Only the last 115 cylinders are indicated in Table 1a.

Because of accuracy limits in the equipment used to collect this data, only increments of 5 mils were recorded for pit depth. As a result, there are several cylinders with the same pit depth measurement, and which, due to data overlaying, appear to be absent in plots of this data (e.g., Figure 1 in Appendix A).

FY95. (See Table 1a.) During FY95, data was collected for 100 thin-wall cylinders at PGDP using the automated P-scanner (Blue 1995). The primary purpose of this effort was to assess "the condition of the more vulnerable portion" of the cylinder population at PGDP (Blue 1995). The cylinders were selected from various yards on the basis of judgement and thus do not constitute a random sample, though they may emulate one.

FY96. (See Table 1b.) During FY96, over 800 cylinders were measured with the P-scanner at Portsmouth and PGDP. Both thin-wall and thick-wall cylinders were measured. At Portsmouth, $10 \%$ of the cylinders that were being relocated were selected using a random number generator. The $10 \%$ evaluation criterion was required by a Consent Decree with the Ohio Environmental Protection Agency. Most of the cylinders measured at PGDP were from the old C-745-G yard and had been set aside as part of relocation efforts performed during FY95 and FY96. They were a subset of the approximately 390 cylinders set aside from the first 3,900 cylinders moved out of that yard. (Because of the selection process, these cylinders are a systematic sample only from the first 3,900 cylinders moved out of $G$ yard.) A few additional cylinders from both C-745-F and C-745-K yard were also measured after selection, mainly on the basis of ease of accessibility with equipment. 
FY97. (See Table 1c.) During FY97, both head/skirt interface and overall minimum wall thickness UT measurements were made, nearly all at Portsmouth, and most for the head/skirt interface area. Head/skirt measurements were made for 115 thick-wall and 232 thin-wall cylinders. The head/skirt measurements were made using manual UT procedures; the overall cylinder body measurements were by P-scanning. (FY97 marks the end of P-scan data used for this report.) The cylinders measured at Portsmouth, which had originally been systematically set aside as part of the $10 \%$ criterion, were randomly selected from those cylinders moved during the year. Originally, it was suggested that approximately 250 cylinders should be measured (Lykins 1996). However, budget constraints allowed only 87 P-scan evaluations. Three P-scan evaluations of thin-wall cylinders were also made at Paducah. These cylinders were located in the north end of the C-745-F yard when they were measured.

FY98. (See Table 1d.) Four populations were sampled in FY98, though the data for only three of the samples is used for this report. The first sample consisted of 40 thin-wall cylinders randomly selected from $\mathrm{K}-1066-\mathrm{K}$ yard at ETTP. These cylinders were chosen from a population of 400 cylinders that were moved to K-1066-E yard during FY98. The second sample consisted of 200 thin-wall cylinders randomly selected from Paducah yards. The Paducah data was representative only of relatively uncorroded locations on each cylinder and therefore is not used for determining either minimum wall thickness or wall loss (and is not indicated in Table 1d. The Paducah cylinders were remeasured in FY99 to determine estimates of the thinnest locations on each cylinder). The third sample consisted of 142 thin-wall and 2 thick-wall cylinders in Portsmouth X-745-C and E yards. Some of the Portsmouth cylinders were also measured in FY96. Finally, 13 30A ( $1 / 2$ " thick) cylinders were measured at Portsmouth. All of these UT measurements were manual.

FY99. (See Table 1e.) There were four sampling efforts in FY99, all using manual UT measurements. One effort consisted of 30 thin-wall cylinders randomly selected from ETTP K-1066-K yard. The cylinders were from a subpopulation of 155 cylinders that could be measured without cylinder movement. All but one of these cylinders was chosen randomly, with the additional one selected by field personnel because of its history of ground/water contact. The second effort was an evaluation of 199 thin-wall cylinders at Paducah (originally slated for measurement in FY98). In the third effort, which was conducted at Portsmouth, measurements were made of 90 thin-wall $48 "$ cylinders. The fourth effort consisted of measurements of 100 model 30A cylinders from a population of 1,825 at Paducah

FY2000. (See Table 1f.) Additional data for FY2000 included manual UT data for 58 thin-wall cylinders from K-1066-K yard at ETTP, 101 thin-wall cylinders at Paducah, and 129 thin-wall and 23 thick-wall cylinders at Portsmouth. At ETTP, the UT procedure involved making nine measurements along the bottom and top (six and twelve o'clock) lines of the cylinders. At PGDP nine measurements were made at various locations. At Portsmouth fourteen measurements were made at approximately equally spaced points on the cylinder ends and bodies, a five additional measurements were made in the areas considered to have the worst corrosion. Head-skirt measurements were also made at Portsmouth for the 23 thick-wall cylinders and for 97 of the thin-wall and cylinders. On each of these cylinders, five measurements were made at the head/skirt interface. Some of the Portsmouth cylinders had also been measured previously.

FY01. (See Table 1g.) FY01 UT measurements at ETTP were made for 24 cylinders in K-1066-E yard and 76 cylinders in K-1066-K yard. At Paducah, 301 48" thin-wall cylinders were measured from (present or former) B, C, F, and K yards, and 99 30A cylinders from A and D yards were measured. At ETTP, the UT procedure involved making four measurement considered to be of the original thickness and five or six additional measurements. The PGDP cylinders were sampled using a random number generator, and the locations of the measurements on the cylinders were as for ETTP. At Portsmouth, 139 thin-wall cylinders and 14 thick-wall cylinders were measured, all from X-745-E yard. Head-skirt measurements were also made at Portsmouth for 14 thick-wall cylinders and for 99 thin-wall cylinders. Locations on the cylinders of the Portsmouth measurements were as in FY2000. Some of the Portsmouth cylinders had been measured before. 
Table 1a. Cylinder Thickness Data Chronology: Data Collected FY92-95

\begin{tabular}{|c|c|c|c|c|c|c|c|c|}
\hline $\begin{array}{l}\text { Year } \\
\text { Measured }\end{array}$ & Site & $\begin{array}{c}\text { Thick- } \\
\text { ness }\end{array}$ & $\begin{array}{l}\text { Skirt } \\
\text { Data? }\end{array}$ & Method & $\begin{array}{c}\text { Current } \\
\text { T/B }\end{array}$ & $\begin{array}{c}\text { Number } \\
\text { Measured }\end{array}$ & $\begin{array}{c}\text { Current } \\
\text { Yard }\end{array}$ & Subgroups \\
\hline 1992 & ETTP & Thin & No & Visual & $\mathrm{B}$ & 2 & K & 2 from $\mathrm{K}$ bottom \\
\hline \multirow[t]{6}{*}{1994} & ETTP & Thin & No & P-Scan & B & 8 & $\mathrm{E}$ & 3 from $\mathrm{K}$ bottom \\
\hline & & & & & & 41 & $\mathrm{~K}$ & 37 from $\mathrm{K}$ bottom \\
\hline & & & & & & 49 & All & 40 from $\mathrm{K}$ bottom \\
\hline & & & & & $\mathrm{T}$ & 8 & $\mathrm{E}$ & 3 from $\mathrm{K}$ bottom \\
\hline & & & & & & 58 & $\mathrm{~K}$ & 3 from $\mathrm{K}$ bottom \\
\hline & & & & & & 66 & All & 6 from $\mathrm{K}$ bottom \\
\hline \multirow[t]{16}{*}{1995} & PGDP & Thin & No & $\mathrm{P}-$ Scan & B & 3 & $\mathrm{C}$ & \\
\hline & & & & & & 16 & $\mathrm{~F}$ & 6 from old $\mathrm{F}$ bottom \\
\hline & & & & & & 13 & G & 7 from old $\mathrm{G}$ bottom \\
\hline & & & & & & 3 & $\mathrm{~K}$ & 1 from old $\mathrm{F}$ bottom \\
\hline & & & & & & 11 & $\mathrm{~L}$ & \\
\hline & & & & & & 1 & M & \\
\hline & & & & & & 2 & $\mathrm{~T}$ & \\
\hline & & & & & & 49 & All & 7 from old $\mathrm{F}$ bottom, 7 from old $\mathrm{G}$ bottom \\
\hline & & & & & $\mathrm{T}$ & 3 & $\mathrm{C}$ & \\
\hline & & & & & & 14 & $\mathrm{~F}$ & 4 from old $\mathrm{F}$ bottom \\
\hline & & & & & & 19 & G & 9 from old $\mathrm{G}$ bottom \\
\hline & & & & & & 1 & $\mathrm{~K}$ & \\
\hline & & & & & & 9 & $\mathrm{~L}$ & \\
\hline & & & & & & 4 & M & 2 from old $\mathrm{G}$ bottom \\
\hline & & & & & & 1 & $\mathrm{~T}$ & \\
\hline & & & & & & 51 & All & 4 from old $\mathrm{F}$ bottom, 11 from old $\mathrm{G}$ bottom \\
\hline
\end{tabular}


Table 1b. Cylinder Thickness Data Chronology: Data Collected FY96

\begin{tabular}{|c|c|c|c|c|c|c|c|}
\hline Site & $\begin{array}{c}\text { Thick- } \\
\text { ness }\end{array}$ & $\begin{array}{l}\text { Skirt } \\
\text { Data? }\end{array}$ & Method & $\begin{array}{c}\text { Curren } \\
\text { T/B }\end{array}$ & $\begin{array}{l}\text { Number of } \\
\text { Cylinders }\end{array}$ & $\begin{array}{c}\text { Current } \\
\text { Yard }\end{array}$ & Subgroups \\
\hline \multirow[t]{14}{*}{ PGDP } & Thin & $\mathrm{N}$ & P-Scan & $\mathrm{B}$ & 18 & $\mathrm{~F}$ & 5 from old $\mathrm{G}$ bottom \\
\hline & & & & & 49 & G & 1 from old $\mathrm{F}$ bottom, 11 from old $\mathrm{G}$ bottom \\
\hline & & & & & 37 & $\mathrm{~K}$ & 10 from old $\mathrm{G}$ bottom \\
\hline & & & & & 4 & $\mathrm{~L}$ & \\
\hline & & & & & 3 & $\mathrm{~S}$ & 2 from old $\mathrm{G}$ bottom \\
\hline & & & & & 27 & $\mathrm{~T}$ & 20 from old $\mathrm{G}$ bottom \\
\hline & & & & & 138 & All & 1 from old $\mathrm{F}$ bottom, 48 from old $\mathrm{G}$ bottom \\
\hline & & & & $\mathrm{T}$ & 19 & $\mathrm{~F}$ & 6 from old $\mathrm{G}$ bottom \\
\hline & & & & & 33 & G & 10 from old $\mathrm{G}$ bottom \\
\hline & & & & & 36 & $\mathrm{~K}$ & 1 from old $\mathrm{F}$ bottom, 12 from old $\mathrm{G}$ bottom \\
\hline & & & & & 2 & $\mathrm{~L}$ & \\
\hline & & & & & 4 & $\mathrm{~S}$ & \\
\hline & & & & & 17 & $\mathrm{~T}$ & 14 from old $\mathrm{G}$ bottom \\
\hline & & & & & 111 & All & 1 from old $\mathrm{F}$ bottom, 42 from old $\mathrm{G}$ bottom \\
\hline \multirow[t]{8}{*}{ PORTS } & Thick & $\mathrm{N}$ & P-Scan & B & 1 & $\mathrm{C}$ & \\
\hline & & & & & 60 & $\mathrm{E}$ & 60 from $\mathrm{E}$ \\
\hline & & & & & 61 & All & 60 from $\mathrm{E}$ \\
\hline & & & & $\mathrm{T}$ & 54 & $\mathrm{E}$ & 54 from $\mathrm{E}$ \\
\hline & Thin & $\mathrm{N}$ & P-Scan & B & 239 & $\mathrm{E}$ & 239 from $\mathrm{E}$ \\
\hline & & & & $\mathrm{T}$ & 1 & $\mathrm{C}$ & \\
\hline & & & & & 232 & $\mathrm{E}$ & 232 from $\mathrm{E}$ \\
\hline & & & & & 233 & All & 232 from $\mathrm{E}$ \\
\hline
\end{tabular}


Table 1c. Cylinder Thickness Data Chronology: Data Collected FY97

\begin{tabular}{|c|c|c|c|c|c|c|c|}
\hline Site & $\begin{array}{c}\text { Thick- } \\
\text { ness }\end{array}$ & $\begin{array}{c}\text { Skirt } \\
\text { Data? }\end{array}$ & Method & $\begin{array}{c}\text { Curren } \\
\text { T/B }\end{array}$ & $\begin{array}{c}\text { Number of } \\
\text { Cylinders }\end{array}$ & $\begin{array}{c}\text { Current } \\
\text { Yard }\end{array}$ & Subgroups \\
\hline PGDP & Thin & $\mathrm{N}$ & P-Scan & B & 3 & $\mathrm{G}$ & 2 from old $\mathrm{G}$ bottom \\
\hline \multirow[t]{6}{*}{ PORTS } & Thick & Y & Manual UT & B & 29 & $\mathrm{E}$ & 29 from $\mathrm{E}$ \\
\hline & & & & $\mathrm{T}$ & 85 & $\mathrm{E}$ & 85 from $\mathrm{E}$ \\
\hline & Thin & $\mathrm{N}$ & P-Scan & B & 40 & $\mathrm{E}$ & 40 from $\mathrm{E}$ \\
\hline & & & & $\mathrm{T}$ & 47 & $\mathrm{E}$ & 47 from $E$ \\
\hline & & Y & Manual UT & B & 113 & $\mathrm{E}$ & 113 from $E$ \\
\hline & & & & $\mathrm{T}$ & 117 & $\mathrm{E}$ & 117 from $E$ \\
\hline
\end{tabular}


Table 1d. Cylinder Thickness Data Chronology: Data Collected FY98

\begin{tabular}{|c|c|c|c|c|c|c|c|}
\hline & Thick- & Skirt & & Currer & Number of & Current & \\
\hline Site & ness & Data? & Method & T/B & Cylinders & Yard & Subgroups \\
\hline \multirow[t]{2}{*}{ ETTP } & Thin & $\mathrm{N}$ & Manual UT & B & 21 & $\mathrm{E}$ & 9 from $\mathrm{K}$ bottom \\
\hline & & & & $\mathrm{T}$ & 19 & $\mathrm{E}$ & 8 from $\mathrm{K}$ bottom \\
\hline \multirow[t]{9}{*}{ PORTS } & $1 / 2^{\prime \prime}$ & $\mathrm{N}$ & Manual UT & B & 12 & $\mathrm{E}$ & 12 from $E$ \\
\hline & Thick & $\mathrm{N}$ & Manual UT & B & 1 & $\mathrm{E}$ & 1 from $E$ \\
\hline & & & & $\mathrm{T}$ & 1 & $\mathrm{E}$ & 1 from $E$ \\
\hline & Thin & $\mathrm{N}$ & Manual UT & B & 63 & $\mathrm{C}$ & \\
\hline & & & & & 5 & $\mathrm{E}$ & 5 from $\mathrm{E}$ \\
\hline & & & & & 68 & All & 5 from $\mathrm{E}$ \\
\hline & & & & $\mathrm{T}$ & 57 & $\mathrm{C}$ & \\
\hline & & & & & 4 & $\mathrm{E}$ & 4 from $E$ \\
\hline & & & & & 61 & All & 4 from $E$ \\
\hline
\end{tabular}


Table 1e. Cylinder Thickness Data Chronology: Data Collected FY99

\begin{tabular}{|c|c|c|c|c|c|c|c|}
\hline Site & $\begin{array}{c}\text { Thick- } \\
\text { ness }\end{array}$ & $\begin{array}{l}\text { Skirt } \\
\text { Data? }\end{array}$ & Method & $\begin{array}{c}\text { Curren } \\
\text { T/B }\end{array}$ & $\begin{array}{l}\text { Number of } \\
\text { Cylinders }\end{array}$ & $\begin{array}{l}\text { Current } \\
\text { Yard }\end{array}$ & Subgroups \\
\hline \multirow[t]{6}{*}{ ETTP } & Thin & $\mathrm{N}$ & Manual UT & B & 10 & $\mathrm{E}$ & 8 from $\mathrm{K}$ bottom \\
\hline & & & & & 5 & $\mathrm{~K}$ & 3 from $\mathrm{K}$ bottom \\
\hline & & & & & 15 & All & 11 from $\mathrm{K}$ bottom \\
\hline & & & & $\mathrm{T}$ & 9 & $\mathrm{E}$ & 2 from $\mathrm{K}$ bottom \\
\hline & & & & & 6 & $\mathrm{~K}$ & 3 from $\mathrm{K}$ bottom \\
\hline & & & & & 15 & All & 5 from $\mathrm{K}$ bottom \\
\hline \multirow[t]{10}{*}{ PGDP } & $1 / 2^{\prime \prime}$ & $\mathrm{N}$ & Manual UT & B & 50 & $\mathrm{~T}$ & \\
\hline & & & & $\mathrm{T}$ & 50 & $\mathrm{~T}$ & \\
\hline & Thin & $\mathrm{N}$ & Manual UT & B & 5 & $\mathrm{C}$ & \\
\hline & & & & & 7 & $\mathrm{~F}$ & 1 from old $\mathrm{G}$ bottom \\
\hline & & & & & 35 & G & 5 from old $\mathrm{F}$ bottom, 15 from old $\mathrm{G}$ bottom \\
\hline & & & & & 8 & $\mathrm{~K}$ & 3 from old $\mathrm{F}$ bottom \\
\hline & & & & & 16 & $\mathrm{~L}$ & \\
\hline & & & & & 2 & M & 1 from old $\mathrm{G}$ bottom \\
\hline & & & & & 36 & $\mathrm{~T}$ & 21 from old $\mathrm{G}$ bottom \\
\hline & & & & & 109 & All & 8 from old $\mathrm{F}$ bottom, 38 from old $\mathrm{G}$ bottom \\
\hline
\end{tabular}


Table 1e-cont'd. Cylinder Thickness Data Chronology: Data Collected FY99

\begin{tabular}{|c|c|c|c|c|c|c|c|}
\hline Site & $\begin{array}{c}\text { Thick- } \\
\text { ness }\end{array}$ & $\begin{array}{r}\text { Skirt } \\
\text { Data? }\end{array}$ & Method & $\begin{array}{c}\text { Current } \\
\text { T/B } \\
\end{array}$ & $\begin{array}{c}\text { Number of } \\
\text { Cylinders }\end{array}$ & $\begin{array}{c}\text { Current } \\
\text { Yard } \\
\end{array}$ & Subgroups \\
\hline \multirow[t]{10}{*}{ PGDP } & Thin & $\mathrm{N}$ & Manual UT & $\mathrm{T}$ & 4 & $\mathrm{C}$ & \\
\hline & & & & & 13 & $\mathrm{~F}$ & 11 from old $\mathrm{F}$ bottom \\
\hline & & & & & 38 & G & 2 from old $\mathrm{F}$ bottom, 21 from old $\mathrm{G}$ bottom \\
\hline & & & & & 1 & K & \\
\hline & & & & & 6 & $\mathrm{~L}$ & \\
\hline & & & & & 1 & $\mathrm{~N}$ & \\
\hline & & & & & 1 & $\mathrm{P}$ & \\
\hline & & & & & 1 & $S$ & 1 from old $\mathrm{G}$ bottom \\
\hline & & & & & 25 & $\mathrm{~T}$ & 18 from old $\mathrm{G}$ bottom \\
\hline & & & & & 90 & All & 13 from old $\mathrm{F}$ bottom, 40 from old $\mathrm{G}$ bottom \\
\hline \multirow[t]{2}{*}{ PORTS } & Thin & $\mathrm{N}$ & Manual UT & B & 53 & $\mathrm{E}$ & 53 from $\mathrm{E}$ \\
\hline & & & & $\mathrm{T}$ & 88 & $\mathrm{E}$ & 88 from $\mathrm{E}$ \\
\hline
\end{tabular}


Table 1f. Cylinder Thickness Data Chronology: Data Collected FY2000

\begin{tabular}{|c|c|c|c|c|c|c|c|}
\hline Site & $\begin{array}{c}\text { Thick- } \\
\text { ness }\end{array}$ & $\begin{array}{l}\text { Skirt } \\
\text { Data? }\end{array}$ & Method & $\begin{array}{c}\text { Curren } \\
\text { T/B }\end{array}$ & $\begin{array}{c}\text { Number of } \\
\text { Cylinders }\end{array}$ & $\begin{array}{l}\text { Current } \\
\text { Yard }\end{array}$ & Subgroups \\
\hline \multirow[t]{6}{*}{ ETTP } & Thin & $\mathrm{N}$ & Manual UT & $\mathrm{B}$ & 6 & $\mathrm{E}$ & 5 from $\mathrm{K}$ bottom \\
\hline & & & & & 25 & $\mathrm{~K}$ & 20 from $\mathrm{K}$ bottom \\
\hline & & & & & 31 & All & 25 from $\mathrm{K}$ bottom \\
\hline & & & & $\mathrm{T}$ & 4 & $\mathrm{E}$ & \\
\hline & & & & & 23 & $\mathrm{~K}$ & 9 from $\mathrm{K}$ bottom \\
\hline & & & & & 27 & All & 9 from $\mathrm{K}$ bottom \\
\hline \multirow[t]{16}{*}{ PGDP } & Thin & $\mathrm{N}$ & Manual UT & B & 1 & $\mathrm{D}$ & \\
\hline & & & & & 8 & $\mathrm{~F}$ & 3 from old $\mathrm{F}$ bottom, 1 from old $\mathrm{G}$ bottom \\
\hline & & & & & 32 & G & 20 from old $\mathrm{G}$ bottom \\
\hline & & & & & 3 & $\mathrm{~K}$ & 1 from old $F$ bottom, 1 from old $G$ bottom \\
\hline & & & & & 4 & $\mathrm{~L}$ & \\
\hline & & & & & 2 & M & 1 from old $\mathrm{F}$ bottom, 1 from old $\mathrm{G}$ bottom \\
\hline & & & & & 1 & $\mathrm{~N}$ & \\
\hline & & & & & 1 & $\mathrm{~T}$ & 1 from old $\mathrm{G}$ bottom \\
\hline & & & & & 52 & All & 5 from old $\mathrm{F}$ bottom, 24 from old $\mathrm{G}$ bottom \\
\hline & & & & $\mathrm{T}$ & 1 & $\mathrm{D}$ & \\
\hline & & & & & 7 & $\mathrm{~F}$ & 6 from old $\mathrm{F}$ bottom \\
\hline & & & & & 33 & G & 2 from old $\mathrm{F}$ bottom, 15 from old $\mathrm{G}$ bottom \\
\hline & & & & & 2 & $\mathrm{~K}$ & 1 from old $\mathrm{F}$ bottom, 1 from old $\mathrm{G}$ bottom \\
\hline & & & & & 2 & $\mathrm{~L}$ & \\
\hline & & & & & 2 & M & 1 from old $\mathrm{F}$ bottom \\
\hline & & & & & 2 & $\mathrm{~N}$ & \\
\hline
\end{tabular}


Table 1f-cont'd. Cylinder Thickness Data Chronology: Data Collected FY2000

\begin{tabular}{|c|c|c|c|c|c|c|c|}
\hline & Thick- & Skirt & & Currel & Number of & Current & \\
\hline Site & ness & Data? & Method & $\mathbf{T} / \mathbf{B}$ & Cylinders & Yard & Subgroups \\
\hline PGDP & Thin & $\mathrm{N}$ & Manual UT & $\mathrm{T}$ & 49 & All & 10 from old $\mathrm{F}$ bottom, 16 from old $\mathrm{G}$ bottom \\
\hline \multirow[t]{12}{*}{ PORTS } & Thick & $\mathrm{N}$ & Manual UT & B & 8 & $\mathrm{E}$ & 8 from $\mathrm{E}$ \\
\hline & & & & $\mathrm{T}$ & 15 & $\mathrm{E}$ & 15 from $\mathrm{E}$ \\
\hline & & Y & Manual UT & B & 8 & $\mathrm{E}$ & 8 from $\mathrm{E}$ \\
\hline & & & & $\mathrm{T}$ & 15 & $\mathrm{E}$ & 15 from $\mathrm{E}$ \\
\hline & Thin & $\mathrm{N}$ & Manual UT & B & 10 & $\mathrm{C}$ & \\
\hline & & & & & 45 & $\mathrm{E}$ & 45 from $\mathrm{E}$ \\
\hline & & & & & 55 & All & 45 from $\mathrm{E}$ \\
\hline & & & & $\mathrm{T}$ & 15 & $\mathrm{C}$ & \\
\hline & & & & & 59 & $\mathrm{E}$ & 59 from $E$ \\
\hline & & & & & 74 & All & 59 from $E$ \\
\hline & & $\mathrm{Y}$ & Manual UT & B & 39 & $\mathrm{E}$ & 39 from $E$ \\
\hline & & & & $\mathrm{T}$ & 48 & $\mathrm{E}$ & 48 from $\mathrm{E}$ \\
\hline
\end{tabular}


Table 1g. Cylinder Thickness Data Chronology: Data Collected FY01

\begin{tabular}{|c|c|c|c|c|c|c|c|}
\hline Site & $\begin{array}{c}\text { Thick- } \\
\text { ness }\end{array}$ & $\begin{array}{l}\text { Skirt } \\
\text { Data? }\end{array}$ & Method & $\begin{array}{c}\text { Current } \\
\text { T/B }\end{array}$ & $\begin{array}{l}\text { Number of } \\
\text { Cylinders }\end{array}$ & $\begin{array}{l}\text { Current } \\
\text { Yard }\end{array}$ & Subgroups \\
\hline \multirow[t]{4}{*}{ ETTP } & Thin & $\mathrm{N}$ & Manual UT & B & 21 & $\mathrm{E}$ & 11 from $\mathrm{K}$ bottom \\
\hline & & & & & 76 & $\mathrm{~K}$ & 53 from $\mathrm{K}$ bottom \\
\hline & & & & & 97 & All & 64 from $\mathrm{K}$ bottom \\
\hline & & & & $\mathrm{T}$ & 3 & $\mathrm{E}$ & 2 from $\mathrm{K}$ bottom \\
\hline \multirow[t]{12}{*}{ PGDP } & $1 / 2^{\prime \prime}$ & $\mathrm{N}$ & Manual UT & B & 2 & $\mathrm{D}$ & \\
\hline & & & & & 49 & $\mathrm{~T}$ & \\
\hline & & & & & 51 & All & \\
\hline & & & & $\mathrm{T}$ & 48 & $\mathrm{~T}$ & \\
\hline & Thin & $\mathrm{N}$ & Manual UT & B & 102 & $\mathrm{C}$ & \\
\hline & & & & & 28 & $\mathrm{~F}$ & 5 from old $\mathrm{F}$ bottom \\
\hline & & & & & 7 & G & 6 from old $\mathrm{F}$ bottom \\
\hline & & & & & 2 & K & 1 from old $\mathrm{F}$ bottom \\
\hline & & & & & 1 & M & 1 from old $\mathrm{F}$ bottom \\
\hline & & & & & 1 & $\mathrm{~S}$ & 1 from old $\mathrm{F}$ bottom \\
\hline & & & & & 1 & $\mathrm{~T}$ & 1 from old $F$ bottom \\
\hline & & & & & 142 & All & 15 from old $\mathrm{F}$ bottom \\
\hline
\end{tabular}


Table 1g-cont'd. Cylinder Thickness Data Chronology: Data Collected FY01

\begin{tabular}{|c|c|c|c|c|c|c|c|}
\hline & Thick- & Skirt & & Curren & Number of & Current & \\
\hline Site & ness & Data? & Method & $\mathbf{T} / \mathbf{B}$ & Cylinders & Yard & Subgroups \\
\hline PGDP & Thin & $\mathrm{N}$ & Manual UT & $\mathrm{T}$ & 99 & $\mathrm{C}$ & \\
\hline & & & & & 45 & $\mathrm{~F}$ & 42 from old $F$ bottom \\
\hline & & & & & 5 & $\mathrm{G}$ & 4 from old $\mathrm{F}$ bottom \\
\hline & & & & & 4 & $\mathrm{~K}$ & 3 from old $\mathrm{F}$ bottom \\
\hline & & & & & 2 & $\mathrm{~L}$ & \\
\hline & & & & & 2 & S & 2 from old $F$ bottom \\
\hline & & & & & 2 & $\mathrm{~T}$ & 2 from old $\mathrm{F}$ bottom \\
\hline & & & & & 159 & All & 53 from old $\mathrm{F}$ bottom \\
\hline PORTS & Thick & $\mathrm{N}$ & Manual UT & B & 6 & $\mathrm{E}$ & 6 from $E$ \\
\hline & & & & $\mathrm{T}$ & 8 & E & 8 from $\mathrm{E}$ \\
\hline & & $\mathrm{Y}$ & Manual UT & B & 6 & E & 6 from $\mathrm{E}$ \\
\hline & & & & $\mathrm{T}$ & 8 & E & 8 from $\mathrm{E}$ \\
\hline & Thin & $\mathrm{N}$ & Manual UT & B & 58 & E & 58 from $\mathrm{E}$ \\
\hline & & & & $\mathrm{T}$ & 81 & $\mathrm{E}$ & 81 from $E$ \\
\hline & & $\mathrm{Y}$ & Manual UT & B & 49 & $\mathrm{E}$ & 49 from $E$ \\
\hline & & & & $\mathrm{T}$ & 50 & $\mathrm{E}$ & 50 from $\mathrm{E}$ \\
\hline
\end{tabular}


Table 1h. Cylinder Thickness Data Chronology: Data Collected FY02

\begin{tabular}{|c|c|c|c|c|c|c|c|}
\hline & Thick- & Skirt & & Currer & Number of & Current & \\
\hline Site & ness & Data? & Method & T/B & Cylinders & Yard & Subgroups \\
\hline \multirow[t]{4}{*}{ ETTP } & Thin & $\mathrm{N}$ & Manual UT & B & 43 & $\mathrm{E}$ & 29 from K bottom \\
\hline & & & & & 53 & $\mathrm{~K}$ & 28 from $\mathrm{K}$ bottom \\
\hline & & & & & 96 & All & 57 from K bottom \\
\hline & & & & $\mathrm{T}$ & 7 & $\mathrm{E}$ & 3 from $\mathrm{K}$ bottom \\
\hline \multirow[t]{14}{*}{ PGDP } & $1 / 2^{\prime \prime}$ & $\mathrm{N}$ & Manual UT & B & 14 & $\mathrm{~T}$ & \\
\hline & & & & $\mathrm{T}$ & 11 & $\mathrm{~T}$ & \\
\hline & Thin & $\mathrm{N}$ & Manual UT & B & 3 & $\mathrm{D}$ & 3 from old $\mathrm{F}$ bottom \\
\hline & & & & & 11 & $\mathrm{~F}$ & 1 from old $\mathrm{F}$ bottom, 1 from old $\mathrm{G}$ bottom \\
\hline & & & & & 24 & G & 5 from old $\mathrm{F}$ bottom, 8 from old $\mathrm{G}$ bottom \\
\hline & & & & & 3 & $\mathrm{~K}$ & \\
\hline & & & & & 4 & $\mathrm{~L}$ & \\
\hline & & & & & 2 & $\mathrm{~T}$ & \\
\hline & & & & & 47 & All & 9 from old $\mathrm{F}$ bottom, 9 from old $\mathrm{G}$ bottom \\
\hline & & & & $\mathrm{T}$ & 2 & $\mathrm{C}$ & \\
\hline & & & & & 4 & $\mathrm{~F}$ & 1 from old $\mathrm{F}$ bottom \\
\hline & & & & & 17 & G & 2 from old $\mathrm{F}$ bottom, 6 from old $\mathrm{G}$ bottom \\
\hline & & & & & 7 & $\mathrm{~K}$ & \\
\hline & & & & & 30 & All & 3 from old $F$ bottom, 6 from old $\mathrm{G}$ bottom \\
\hline
\end{tabular}


Table 1h-cont'd. Cylinder Thickness Data Chronology: Data Collected FY02

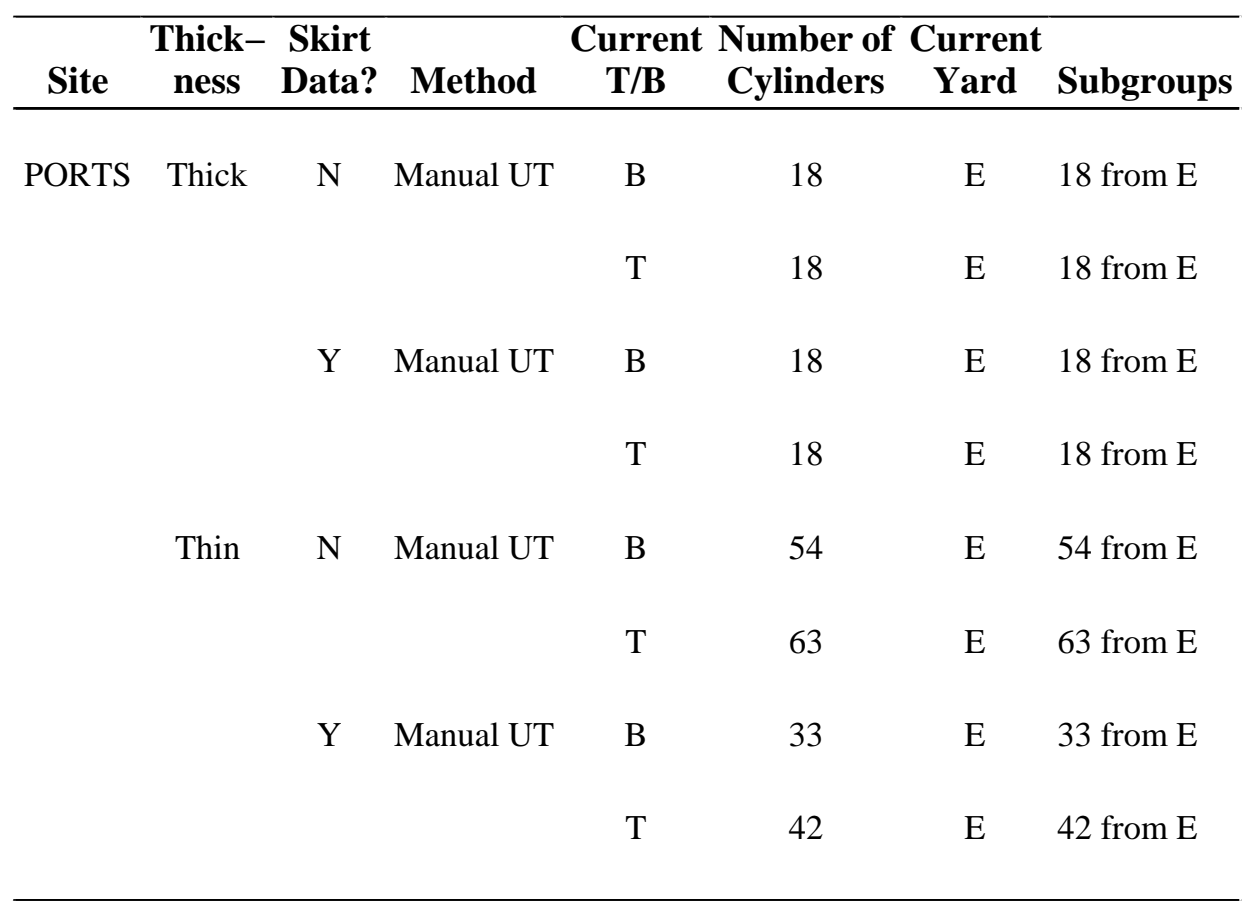


FY02. (See Table 1h.) FY02 UT measurements were made for 10448 " thin-wall cylinders sampled at ETTP. At PGDP, 77 thin-wall cylinders and 25 30A cylinders were sampled and measured. At Portsmouth, 117 thin-wall and 36 thick-wall were measured. The ETTP and PGDP measurements were located on the cylinders as in FY01. Head-skirt measurements were also made at Portsmouth for the 36 thick-wall cylinders and for 75 of the thin-wall cylinders. Locations on the cylinders of the Portsmouth measurements were as in FY2000.

\subsection{Summary of Data by Subpopulation}

As in Section 3.1, this section also summarizes UT measurement data. However, in this section the data is classified by subpopulation (i.e., cylinder group) rather than by year of data collection. The subpopulations, after a few refinements discussed in Section 4, are ultimately used in the corrosion regression models. They are defined on the basis of knowledge about future storage conditions, experience with cylinder storage, consideration of the amount of thickness data that has been collected, and decisions about what classification would be most useful. Tables $2 \mathrm{a}-\mathrm{d}$ list the subpopulations along with total population counts and counts by fiscal year of the thickness measurements.

Tables 2a-c are for cylinder subpopulations at ETTP, PGDP, and PORTS, respectively, for which one or more wall-thickness measurements have been made. Table $2 \mathrm{~d}$ lists other cylinder subpopulations for which no wall-thickness measurements have been made. For projections about numbers of cylinders that will fail the various thickness criteria, these subpopulations are grouped with subpopulations that do have data and are thus used to fit the indirect or direct corrosion models. Total population counts are also listed in the tables for cylinders of known age and for cylinders not painted in the last ten years, which are considered at risk in the cylinder corrosion models. (Cylinders painted in the last ten years are assumed to be not at risk.) Cylinders of unknown age cannot be used in the (age-based) corrosion models. Thus projections of numbers of cylinders failing various thickness criteria are ultimately based on population counts for unpainted cylinders of known age.

It is often useful to focus on worst cases. Estimates of corrosion performance for worst-case cylinder groups are lower bounds for the performance expected from other cylinders. Bottom-row storage conditions are generally worse than top. Various cylinder yards, such as the former (unrefurbished) G-yard at Paducah, are generally considered to be worst-case yards. K-yard is considered worst at ETTP, and Eyard is considered worst at Portsmouth, though there is probably less of a difference between yards at Portsmouth than at the other sites. ${ }^{6}$ The worst cases are used in defining the classifications for thin-wall cylinders.

Most of the wall-thickness data available for this report is for thin-wall cylinders (as opposed to thick-wall or $1 / 2 "$ nominal thickness) cylinders. Also, most of the wall-thickness data is for wall areas on the cylinder body as opposed to the head/skirt interface. Some data, though considerably less, is available for $1 / 2$ " and thick-wall cylinders and for the head/skirt interface area. Therefore, although statistics for $1 / 2$ " and thickwall cylinders and the head/skirt interface area are broken down by site in Tables $2 \mathrm{a}-\mathrm{d}$, single classes for all three sites are used for this data in the corrosion modeling. These cylinders are classified by top/bottom status in the corrosion models, however, except for the thick-wall head/skirt data, which is combined even for the top and bottom cylinders.

For thin-wall cylinders, PGDP former G-yard bottom (worst case) cylinders are taken as a separate class, as are ETTP K-yard bottom cylinders. PGDP bottom-row thin-wall cylinders not from former G-yard are then taken as a separate class (not worst-case), as are PGDP top-row thin-wall cylinders. Because of limits on data availability, all (both top and bottom) ETTP thin-wall cylinders other than K-yard bottom cylinders

${ }^{6}$ Roger McDermott, Theta Technologies, Inc., personal communication. 
are taken as a separate class. Also because of limits on data availability, Portsmouth thin-wall cylinders are classified by top/bottom status only, not by yard, with bottom cylinders representing the worst-case.

The best way to classify cylinder can change over time. Previously all ETTP cylinders were classed together. With additional data available for this edition of the report, ETTP, K-yard bottom cylinders (considered to represent the worst-case) are treated as a separate class. Former F-yard at PGDP is considered to represent the second-worst case at PGDP, next to former G-yard, ${ }^{7}$ and former F-yard cylinders may be separately classified in a future edition of this report. For now, however, there does not seem to be sufficient data to warrant modeling F yard separately.

Cylinders at Portsmouth are stored in one of two yards, C-yard and E-yard. Although C-yard has more cylinders, most of the Portsmouth wall thickness data is for cylinders from E-yard, which is considered the worst case. Therefore, $\mathrm{C}$ and E-yard thin-wall cylinders are classified separately in Tables $2 \mathrm{c}$ and $2 \mathrm{~d}$, but for the corrosion and wall-thickness modeling, $\mathrm{C}$ and $\mathrm{E}$-yard cylinders are grouped together, and only the top/bottom status is used to classify Portsmouth thin-wall cylinders.

The location of a cylinder - its site and yard — and its top/bottom status can change over the cylinder's lifetime. Complete location histories of cylinders are not generally available in the CID or in any other electronic format, and even if the histories were available, incorporating them into a corrosion model would be complicated and difficult. For many cylinders, even when former locations are recorded, the corresponding old top/bottom status is not. Thus some guesswork and approximations are used in classifying cylinders on the basis of their location histories. In this report, the "location" of a cylinder will refer in theory to where the cylinder was stored longest, but because the longest-storage locations are not determinable exactly for every cylinder, "location" will in practice be a best guess of where a cylinder was stored longest. Sometimes current top/bottom statuses are substituted for missing former top/bottom statuses. Thus, the cylinder classification used for this report is a rough approximation to a more ideal classification.

For the data in this report, except for head/skirt interface data, all UT measurements made before FY98 were by P-scanning and all UT measurements in FY98 and later were by manual UT scans. All of the head/skirt measurements were made by manual UT scans. However, P-scan and manual UT measurements seem to differ systematically (see Section 4, Table 4). Because the P-scan measurements were all made before any of the manual UT measurements, the systematic difference would tend to skew corrosion trend estimates, if the data were combined into a single uncorrected analysis. Therefore, the P-scan (pre-FY98) and manual UT data are treated as coming from separate statistical distributions (except, as described below, for thick-wall cylinders), even though the underlying cylinder groups are the same. For example, Pscan data for ETTP thin-wall cylinders and manual UT data for ETTP thin-wall cylinders are modeled as separate groups. The cylinder thickness data is divided into pre-FY98 and FY98 and later groups, and, for the manual UT data, further divided by site, yard, and top/bottom status.

In previous editions of this report, for PGDP cylinders, P-scan data was grouped along with manual UT data. A partial rationale for combining the P-scan and manual UT PGDP data was that much of the PGDP P-scan data (namely the FY96 data) was adjusted by adding 15 mils to the measured maximum pit depths (see Lyon 2000, p 17). On the other hand, the ETTP data Table 4 shows that minimum wall thickness are actually under-estimated by the P-scan method (by 50 mils, on average) and thus the pit depths themselves are over-estimated. An analysis of Portsmouth cylinders measured in FY96 and again in FY98-01 leads to similar conclusions. So the correction for the PGDP P-scan data to make it more like manual UT data may

\footnotetext{
${ }^{7}$ The top and bottom rows of PGDP F yard were interchanged in FY92 when all bottom row cylinder chocks were replaced, concrete chocks replacing wood. Each row was also relocated south one row. It is likely that some of these bottom row cylinders were in water contact for extended periods of time, although none are now, and conditions in the F-yard are considered to have been better than G-yard conditions.
} 
actually be in the wrong direction. In any case, for this report, PGDP P-scan data is also grouped separately in the corrosion modeling.

None of the 1/2" (30" dia.) cylinder scans were done by P-scanning. However, a substantial portion of the thick-wall cylinder measurements were made in FY96 by P-scanning. Furthermore, there is not sufficient thick-wall data to model the P-scan and manual UT measurements separately. So, for thick-wall cylinders, the P-scan and manual UT data is combined, as in previous versions of this report.

Over the last few years, manual UT data has become preponderant in the database. Furthermore, sampling methods in the earlier years were generally much more purposive (e.g., to deliberately examine suspect cylinders). Subsequent sampling tended to be more random. Thus the P-scan data is incorporated into the corrosion modeling, but as separate data classes, and conclusions from the P-scan data are deprecated in the interpretation, just as the P-scan method itself has been deprecated in the cylinder monitoring.

ETTP Thin-Wall Cylinders. There are 4,721 thin-wall cylinders at ETTP. Of these 4,036 have not been painted in the last ten years and are of known age ranging (in 2003) from 11 to 47 years. The bottom-rows of K-yard (K-1066-K yard) are considered to represent worst-case storage conditions at ETTP. A large portion of K-yard cylinders were previously stored in ground contact at K-1066-G yard, starting about 1966. They were relocated to K-yard in 1983 (Barber et al. 1994), where they are stored either in top or bottom rows. According to CID records (as of June 2003), 1,512 ETTP cylinders are classified as having spent the substantial part of their lifetimes at K-yard bottom. (Of these, 1,270 are currently stored in top rows, and 242 are currently stored in bottom rows.) Of the 1,512, 1,167 are unpainted and of known age. There are 2,870 (1,190 bottom and 1,889 top) other currently unpainted cylinders at ETTP, of which 2,869 are of known age. Table $2 \mathrm{a}$ inventories the P-scan and manual UT cylinder thickness data available for ETTP.

Paducah Thin-Wall Cylinders. According to the CID, there are 35,599 thin-wall cylinders at Paducah of which 32,213 are of known age and have not been painted in the last ten years. The ages range from 4 to 47 years. The following three populations of Paducah thin-wall cylinders were defined for the purpose of corrosion modeling: (1) PGDP G-yard (i.e., C-745-G yard), bottom-row, thin-wall cylinders, (2) all other PGDP bottom-row, thin-wall cylinders, and (3) all PGDP, top-row, thin-wall cylinders (including G yard cylinders). All three populations are combined for the purpose of modeling with the P-scan data. The subpopulation of cylinders classified as coming from $\mathrm{G}$ yard actually consists of those cylinders that were originally in C-745-G yard prior to construction of the new yard. A painting program was initiated for cylinders moved from C-745-G to C-745-S yard in FY96. All 2,168 cylinders in C-745-S were painted during FY96-97. There are currently 3,907 cylinder classified as from former G-yard bottom, but only 1,983 are unpainted (all of known age). Of these 1,983, 1,047 are currently stored in bottom rows, and 936 are currently stored in top rows. There are 16,809 other (i.e, not from former G-yard) bottom-row cylinders at Paducah, of which 15,334 are unpainted and of known age. There are 15,603 top-row cylinders, of which 14,896 are unpainted and of known age. Table $2 b$ inventories the thin-wall cylinder thickness data available for PGDP cylinders.

Portsmouth Thin-Wall Cylinders. According to the CID, there are 17,269 thin-wall cylinders at Portsmouth, all but four of which are of known age and have not been painted in the last ten years. The ages range from 7 to 47 years. The CID classifies Portsmouth cylinders as coming from two yards, either $\mathrm{C}$ or E. Cylinders in both yards are stacked in two tiers. Prior to FY96, there were four cylinder yards at Portsmouth, designated as X-745-A, X-745-C, X-745-E, and X-745-F. The X-745-A and X-745-C yards were essentially the same yard, C-yard, but were separated into different sections. 
Table 2a. ETTP Cylinder Population and Thickness Data Inventory

\begin{tabular}{|c|c|c|c|c|c|c|c|c|c|c|c|c|}
\hline Thickness & $\begin{array}{l}\text { Skirt } \\
\text { Data? }\end{array}$ & $\begin{array}{c}\text { Current } \\
\text { T/B }\end{array}$ & Subgroups & $\begin{array}{l}\text { Pop. } \\
\text { Count }\end{array}$ & Unpainted & $\begin{array}{l}\text { Unpainted, } \\
\text { Age Known }\end{array}$ & $\begin{array}{c}\text { Min } \\
\text { Age } \\
(\mathbf{2 0 0 3})\end{array}$ & $\begin{array}{c}\text { Mean } \\
\text { Age } \\
(\mathbf{2 0 0 3})\end{array}$ & $\begin{array}{c}\text { Max } \\
\text { Age } \\
(\mathbf{2 0 0 3})\end{array}$ & $\begin{array}{c}\text { FY } \\
\text { Measured }\end{array}$ & $\begin{array}{l}\text { Number } \\
\text { Measured }\end{array}$ & $\begin{array}{c}\text { Current } \\
\text { Yards }\end{array}$ \\
\hline \multirow[t]{25}{*}{ Thin } & No & B & - & 1,320 & 1,191 & 1,190 & 11 & 31.3 & 47 & 1994 & 9 & $\mathrm{E}, \mathrm{K}$ \\
\hline & & & & & & & & & & 1998 & 12 & E \\
\hline & & & & & & & & & & 1999 & 4 & $\mathrm{E}, \mathrm{K}$ \\
\hline & & & & & & & & & & 2000 & 6 & $\mathrm{E}, \mathrm{K}$ \\
\hline & & & & & & & & & & 2001 & 33 & $\mathrm{E}, \mathrm{K}$ \\
\hline & & & & & & & & & & 2002 & 39 & $\mathrm{E}, \mathrm{K}$ \\
\hline & & & K-bottom & 1,270 & 1,034 & 1,034 & 12 & 42.0 & 47 & 1992 & 2 & K \\
\hline & & & & & & & & & & 1994 & 40 & E, K \\
\hline & & & & & & & & & & 1998 & 9 & $\mathrm{E}$ \\
\hline & & & & & & & & & & 1999 & 11 & E, K \\
\hline & & & & & & & & & & 2000 & 25 & E, K \\
\hline & & & & & & & & & & 2001 & 64 & $\mathrm{E}, \mathrm{K}$ \\
\hline & & & & & & & & & & 2002 & 57 & $\mathrm{E}, \mathrm{K}$ \\
\hline & & $\mathrm{T}$ & - & 1,889 & 1,679 & 1,679 & 21 & 35.4 & 47 & 1994 & 60 & $\mathrm{E}, \mathrm{K}$ \\
\hline & & & & & & & & & & 1998 & 11 & E \\
\hline & & & & & & & & & & 1999 & 10 & E, K \\
\hline & & & & & & & & & & 2000 & 18 & $\mathrm{E}, \mathrm{K}$ \\
\hline & & & & & & & & & & 2001 & 1 & E \\
\hline & & & & & & & & & & 2002 & 4 & $\mathrm{E}$ \\
\hline & & & K-bottom & 242 & 133 & 133 & 40 & 45.1 & 46 & 1994 & 6 & $\mathrm{E}, \mathrm{K}$ \\
\hline & & & & & & & & & & 1998 & 8 & $\mathrm{E}$ \\
\hline & & & & & & & & & & 1999 & 5 & $\mathrm{E}, \mathrm{K}$ \\
\hline & & & & & & & & & & 2000 & 9 & K \\
\hline & & & & & & & & & & 2001 & 2 & E \\
\hline & & & & & & & & & & 2002 & 3 & E \\
\hline
\end{tabular}


Table 2b. PGDP Cylinder Population and Thickness Data Inventory

\begin{tabular}{|c|c|c|c|c|c|c|c|c|c|c|c|c|}
\hline Thickness & $\begin{array}{c}\text { Skirt } \\
\text { Data? }\end{array}$ & $\begin{array}{c}\text { Current } \\
\text { T/B }\end{array}$ & Subgroups & $\begin{array}{l}\text { Pop. } \\
\text { Count }\end{array}$ & Unpainted & $\begin{array}{l}\text { Unpainted, } \\
\text { Age Known }\end{array}$ & $\begin{array}{c}\text { Min } \\
\text { Age } \\
(\mathbf{2 0 0 3 )}\end{array}$ & $\begin{array}{c}\text { Mean } \\
\text { Age } \\
(2003)\end{array}$ & $\begin{array}{c}\text { Max } \\
\text { Age } \\
(\mathbf{2 0 0 3 )}\end{array}$ & $\begin{array}{c}\text { FY } \\
\text { Measured }\end{array}$ & $\begin{array}{c}\text { Number } \\
\text { Measured }\end{array}$ & $\begin{array}{c}\text { Current } \\
\text { Yards }\end{array}$ \\
\hline \multirow[t]{5}{*}{$1 / 2 "$} & No & B & - & 926 & 926 & 926 & 49 & 49.0 & 49 & 1999 & 50 & $\mathrm{~T}$ \\
\hline & & & & & & & & & & 2001 & 51 & $\mathrm{~T}, \mathrm{D}$ \\
\hline & & & & & & & & & & 2002 & 14 & $\mathrm{~T}$ \\
\hline & & & & & & & & & & 2001 & 48 & $\mathrm{~T}$ \\
\hline & & & & & & & & & & 2002 & 11 & $\mathrm{~T}$ \\
\hline \multirow[t]{16}{*}{ Thin } & No & B & - & 16,089 & 15,347 & 15,334 & 4 & 20.5 & 47 & 1995 & 42 & $\mathrm{~K}, \mathrm{~T}, \mathrm{C}, \mathrm{F}, \mathrm{G}, \mathrm{L}, \mathrm{M}$ \\
\hline & & & & & & & & & & 1996 & 90 & $\mathrm{~K}, \mathrm{~T}, \mathrm{~F}, \mathrm{G}, \mathrm{L}, \mathrm{S}$ \\
\hline & & & & & & & & & & 1997 & 1 & $\mathrm{G}$ \\
\hline & & & & & & & & & & 2000 & 28 & $\mathrm{~K}, \mathrm{D}, \mathrm{F}, \mathrm{G}, \mathrm{L}, \mathrm{M}, \mathrm{N}$ \\
\hline & & & & & & & & & & 2001 & 142 & $\mathrm{~K}, \mathrm{~T}, \mathrm{C}, \mathrm{F}, \mathrm{G}, \mathrm{M}, \mathrm{S}$ \\
\hline & & & & & & & & & & 2002 & 38 & $\mathrm{~K}, \mathrm{~T}, \mathrm{D}, \mathrm{F}, \mathrm{G}, \mathrm{L}$ \\
\hline & & & Old G-Btm. & 2,016 & 1,047 & 1,047 & 11 & 36.2 & 44 & 1995 & 7 & $\mathrm{G}$ \\
\hline & & & & & & & & & & 1996 & 48 & $\mathrm{~K}, \mathrm{~T}, \mathrm{~F}, \mathrm{G}, \mathrm{S}$ \\
\hline & & & & & & & & & & 1997 & 2 & $\mathrm{G}$ \\
\hline & & & & & & & & & & 1999 & 38 & $\mathrm{~T}, \mathrm{~F}, \mathrm{G}, \mathrm{M}$ \\
\hline & & & & & & & & & & 2000 & 24 & $\mathrm{~K}, \mathrm{~T}, \mathrm{~F}, \mathrm{G}, \mathrm{M}$ \\
\hline & & & & & & & & & & 2002 & 9 & $\mathrm{~F}, \mathrm{G}$ \\
\hline & & $\mathrm{T}$ & - & 15,603 & 14,901 & 14,896 & 4 & 21.0 & 47 & 1995 & 40 & $\mathrm{~K}, \mathrm{~T}, \mathrm{C}, \mathrm{F}, \mathrm{G}, \mathrm{L}, \mathrm{M}$ \\
\hline & & & & & & & & & & 1996 & 69 & $\mathrm{~K}, \mathrm{~T}, \mathrm{~F}, \mathrm{G}, \mathrm{L}, \mathrm{S}$ \\
\hline & & & & & & & & & & 1999 & 50 & $\mathrm{~K}, \mathrm{~T}, \mathrm{C}, \mathrm{F}, \mathrm{G}, \mathrm{L}, \mathrm{N}, \mathrm{P}$ \\
\hline & & & & & & & & & & 2000 & 33 & $\mathrm{~K}, \mathrm{D}, \mathrm{F}, \mathrm{G}, \mathrm{L}, \mathrm{M}, \mathrm{N}$ \\
\hline
\end{tabular}


Table 2b-cont'd. PGDP Cylinder Population and Thickness Data Inventory

\begin{tabular}{|c|c|c|c|c|c|c|c|c|c|c|c|c|}
\hline Thickness & $\begin{array}{l}\text { Skirt } \\
\text { Data? }\end{array}$ & $\begin{array}{c}\text { Current } \\
\text { T/B } \\
\end{array}$ & Subgroups & $\begin{array}{l}\text { Pop. } \\
\text { Count }\end{array}$ & Unpainted & $\begin{array}{l}\text { Unpainted, } \\
\text { Age Known }\end{array}$ & $\begin{array}{c}\text { Min } \\
\text { Age } \\
(\mathbf{2 0 0 3}) \\
\end{array}$ & $\begin{array}{c}\text { Mean } \\
\text { Age } \\
(\mathbf{2 0 0 3 )} \\
\end{array}$ & $\begin{array}{c}\text { Max } \\
\text { Age } \\
(\mathbf{2 0 0 3}) \\
\end{array}$ & $\begin{array}{c}\text { FY } \\
\text { Measured }\end{array}$ & $\begin{array}{c}\text { Number } \\
\text { Measured }\end{array}$ & $\begin{array}{c}\text { Current } \\
\text { Yards }\end{array}$ \\
\hline \multirow[t]{5}{*}{ Thin } & No & $\mathrm{T}$ & Old G-Btm. & 1,891 & 936 & 936 & 12 & 38.4 & 44 & 1995 & 11 & $\mathrm{G}, \mathrm{M}$ \\
\hline & & & & & & & & & & 1996 & 42 & $\mathrm{~K}, \mathrm{~T}, \mathrm{~F}, \mathrm{G}$ \\
\hline & & & & & & & & & & 1999 & 40 & $\mathrm{~T}, \mathrm{G}, \mathrm{S}$ \\
\hline & & & & & & & & & & 2000 & 16 & $\mathrm{~K}, \mathrm{G}$ \\
\hline & & & & & & & & & & 2002 & 6 & G \\
\hline
\end{tabular}


Table 2c. Portsmouth Cylinder Population and Thickness Data Inventory

\begin{tabular}{|c|c|c|c|c|c|c|c|c|c|c|c|c|}
\hline Thickness & $\begin{array}{c}\text { Skirt } \\
\text { Data? }\end{array}$ & $\begin{array}{c}\text { Current } \\
\text { T/B }\end{array}$ & Subgroups & $\begin{array}{c}\text { Pop. } \\
\text { Count }\end{array}$ & Unpainted & $\begin{array}{l}\text { Unpainted, } \\
\text { Age Known }\end{array}$ & $\begin{array}{c}\text { Min } \\
\text { Age } \\
(\mathbf{2 0 0 3 )}\end{array}$ & $\begin{array}{c}\text { Mean } \\
\text { Age } \\
(\mathbf{2 0 0 3 )}\end{array}$ & $\begin{array}{c}\text { Max } \\
\text { Age } \\
(\mathbf{2 0 0 3})\end{array}$ & $\begin{array}{c}\text { FY } \\
\text { Measured }\end{array}$ & $\begin{array}{c}\text { Number } \\
\text { Measured }\end{array}$ & $\begin{array}{c}\text { Current } \\
\text { Yards }\end{array}$ \\
\hline $1 / 2^{\prime \prime}$ & No & B & $\mathrm{E}$ & 252 & 252 & 252 & 3 & 48.6 & 49 & 1998 & 12 & $\mathrm{E}$ \\
\hline \multirow[t]{19}{*}{ Thick } & No & B & - & 2 & 2 & 1 & 49 & 49.0 & 49 & 1996 & 1 & $\mathrm{C}$ \\
\hline & & & $\mathrm{E}$ & 648 & 648 & 648 & 23 & 49.3 & 52 & 1996 & 60 & $\mathrm{E}$ \\
\hline & & & & & & & & & & 1998 & 1 & $\mathrm{E}$ \\
\hline & & & & & & & & & & 2000 & 8 & $\mathrm{E}$ \\
\hline & & & & & & & & & & 2001 & 6 & $\mathrm{E}$ \\
\hline & & & & & & & & & & 2002 & 18 & $\mathrm{E}$ \\
\hline & & $\mathrm{T}$ & $\mathrm{E}$ & 615 & 615 & 615 & 23 & 49.3 & 52 & 1996 & 54 & $\mathrm{E}$ \\
\hline & & & & & & & & & & 1998 & 1 & $\mathrm{E}$ \\
\hline & & & & & & & & & & 2000 & 15 & $\mathrm{E}$ \\
\hline & & & & & & & & & & 2001 & 8 & $\mathrm{E}$ \\
\hline & & & & & & & & & & 2002 & 18 & $\mathrm{E}$ \\
\hline & Yes & B & $\mathrm{E}$ & 648 & 648 & 648 & 23 & 49.3 & 52 & 1997 & 29 & $\mathrm{E}$ \\
\hline & & & & & & & & & & 2000 & 8 & $\mathrm{E}$ \\
\hline & & & & & & & & & & 2001 & 6 & $\mathrm{E}$ \\
\hline & & & & & & & & & & 2002 & 18 & $\mathrm{E}$ \\
\hline & & $\mathrm{T}$ & $\mathrm{E}$ & 615 & 615 & 615 & 23 & 49.3 & 52 & 1997 & 85 & $\mathrm{E}$ \\
\hline & & & & & & & & & & 2000 & 15 & $\mathrm{E}$ \\
\hline & & & & & & & & & & 2001 & 8 & $\mathrm{E}$ \\
\hline & & & & & & & & & & 2002 & 18 & $\mathrm{E}$ \\
\hline \multirow[t]{2}{*}{ Thin } & No & B & - & 7,345 & 7,345 & 7,342 & 7 & 18.9 & 44 & 1998 & 63 & $\mathrm{C}$ \\
\hline & & & & & & & & & & 2000 & 10 & $\mathrm{C}$ \\
\hline
\end{tabular}


Table 2c-cont'd. Portsmouth Cylinder Population and Thickness Data Inventory

\begin{tabular}{|c|c|c|c|c|c|c|c|c|c|c|c|c|}
\hline Thickness & $\begin{array}{l}\text { Skirt } \\
\text { Data? }\end{array}$ & $\begin{array}{c}\text { Current } \\
\text { T/B }\end{array}$ & Subgroups & $\begin{array}{l}\text { Pop. } \\
\text { Count }\end{array}$ & Unpainted & $\begin{array}{l}\text { Unpainted, } \\
\text { Age Known }\end{array}$ & $\begin{array}{c}\text { Min } \\
\text { Age } \\
(\mathbf{2 0 0 3})\end{array}$ & $\begin{array}{c}\text { Mean } \\
\text { Age } \\
(2003) \\
\end{array}$ & $\begin{array}{c}\text { Max } \\
\text { Age } \\
(\mathbf{2 0 0 3}) \\
\end{array}$ & $\begin{array}{c}\text { FY } \\
\text { Measured }\end{array}$ & $\begin{array}{c}\text { Number } \\
\text { Measured }\end{array}$ & $\begin{array}{c}\text { Current } \\
\text { Yards }\end{array}$ \\
\hline \multirow[t]{25}{*}{ Thin } & No & B & $\mathrm{E}$ & 1,423 & 1,423 & 1,423 & 13 & 39.9 & 47 & 1996 & 239 & $\mathrm{E}$ \\
\hline & & & & & & & & & & 1997 & 40 & $\mathrm{E}$ \\
\hline & & & & & & & & & & 1998 & 5 & $\mathrm{E}$ \\
\hline & & & & & & & & & & 1999 & 53 & $\mathrm{E}$ \\
\hline & & & & & & & & & & 2000 & 45 & $\mathrm{E}$ \\
\hline & & & & & & & & & & 2001 & 58 & $\mathrm{E}$ \\
\hline & & & & & & & & & & 2002 & 54 & $\mathrm{E}$ \\
\hline & & $\mathrm{T}$ & - & 7,118 & 7,118 & 7,117 & 7 & 19.0 & 41 & 1996 & 1 & $\mathrm{C}$ \\
\hline & & & & & & & & & & 1998 & 57 & $\mathrm{C}$ \\
\hline & & & & & & & & & & 2000 & 15 & $\mathrm{C}$ \\
\hline & & & $\mathrm{E}$ & 1,383 & 1,383 & 1,383 & 13 & 41.2 & 47 & 1996 & 232 & $\mathrm{E}$ \\
\hline & & & & & & & & & & 1997 & 47 & $\mathrm{E}$ \\
\hline & & & & & & & & & & 1998 & 4 & $\mathrm{E}$ \\
\hline & & & & & & & & & & 1999 & 88 & $\mathrm{E}$ \\
\hline & & & & & & & & & & 2000 & 59 & $\mathrm{E}$ \\
\hline & & & & & & & & & & 2001 & 81 & $\mathrm{E}$ \\
\hline & & & & & & & & & & 2002 & 63 & $\mathrm{E}$ \\
\hline & Yes & B & $\mathrm{E}$ & 1,063 & 1,063 & 1,063 & 46 & 46.1 & 47 & 1997 & 113 & $\mathrm{E}$ \\
\hline & & & & & & & & & & 2000 & 39 & $\mathrm{E}$ \\
\hline & & & & & & & & & & 2001 & 49 & $\mathrm{E}$ \\
\hline & & & & & & & & & & 2002 & 33 & $\mathrm{E}$ \\
\hline & & $\mathrm{T}$ & $\mathrm{E}$ & 1,063 & 1,063 & 1,063 & 46 & 46.1 & 47 & 1997 & 117 & $\mathrm{E}$ \\
\hline & & & & & & & & & & 2000 & 48 & $\mathrm{E}$ \\
\hline & & & & & & & & & & 2001 & 50 & $\mathrm{E}$ \\
\hline & & & & & & & & & & 2002 & 42 & $\mathrm{E}$ \\
\hline
\end{tabular}


Table 2d. Site-Specific Cylinder Groupings Without Thickness Data

\begin{tabular}{|c|c|c|c|c|c|c|c|c|c|c|}
\hline Site & Thickness & $\begin{array}{c}\text { Skirt } \\
\text { Data? }\end{array}$ & $\begin{array}{c}\text { Current } \\
\text { T/B }\end{array}$ & Subgroups & $\begin{array}{c}\text { Pop. } \\
\text { Count }\end{array}$ & Unpainted & $\begin{array}{l}\text { Unpainted, } \\
\text { Age Known }\end{array}$ & $\begin{array}{c}\text { Min } \\
\text { Age } \\
(\mathbf{2 0 0 3 )}\end{array}$ & $\begin{array}{c}\text { Mean } \\
\text { Age } \\
(2003)\end{array}$ & $\begin{array}{c}\text { Max } \\
\text { Age } \\
(\mathbf{2 0 0 3 )}\end{array}$ \\
\hline \multirow[t]{10}{*}{ ETTP } & $1 / 2^{\prime \prime}$ & No & B & - & 387 & 387 & 346 & 49 & 49 & 49 \\
\hline & & & $\mathrm{T}$ & - & 339 & 339 & 291 & 49 & 49 & 49 \\
\hline & Thick & No & B & - & 189 & 189 & 187 & 41 & 48 & 52 \\
\hline & & & $\mathrm{T}$ & - & 204 & 204 & 204 & 41 & 49 & 52 \\
\hline & & Yes & B & - & 189 & 189 & 187 & 41 & 48 & 52 \\
\hline & & & $\mathrm{T}$ & - & 204 & 204 & 204 & 41 & 49 & 52 \\
\hline & Thin & Yes & B & - & 330 & 203 & 202 & 21 & 45 & 47 \\
\hline & & & & $\mathrm{K}$-bottom & 514 & 279 & 279 & 46 & 46 & 47 \\
\hline & & & $\mathrm{T}$ & - & 430 & 220 & 220 & 24 & 46 & 47 \\
\hline & & & & K-bottom & 219 & 110 & 110 & 46 & 46 & 46 \\
\hline \multirow[t]{6}{*}{ PGDP } & Thick & No & B & - & 223 & 223 & 214 & 10 & 42 & 52 \\
\hline & & & $\mathrm{T}$ & - & 143 & 143 & 132 & 10 & 38 & 52 \\
\hline & & Yes & B & - & 223 & 223 & 214 & 10 & 42 & 52 \\
\hline & & & $\mathrm{T}$ & - & 143 & 143 & 132 & 10 & 38 & 52 \\
\hline & Thin & Yes & B & - & 344 & 344 & 342 & 17 & 45 & 47 \\
\hline & & & $\mathrm{T}$ & - & 317 & 317 & 316 & 22 & 45 & 47 \\
\hline \multirow[t]{6}{*}{ PORTS } & $1 / 2^{\prime \prime}$ & No & B & - & 81 & 81 & 80 & 3 & 48 & 49 \\
\hline & & & $\mathrm{T}$ & - & 78 & 78 & 77 & 3 & 48 & 49 \\
\hline & & & & $\mathrm{E}$ & 1 & 1 & 1 & 49 & 49 & 49 \\
\hline & Thick & Yes & B & - & 2 & 2 & 1 & 49 & 49 & 49 \\
\hline & Thin & Yes & B & - & 598 & 598 & 598 & 21 & 23 & 24 \\
\hline & & & $\mathrm{T}$ & - & 575 & 575 & 575 & 21 & 23 & 24 \\
\hline
\end{tabular}


Cylinders at Portsmouth were moved from single row storage to a two-tiered arrangement around 1976. Prior to that, there were no top row cylinders at Portsmouth. Thus, current "top" row cylinders at Portsmouth have been in the top row for at most about 27 (=2003-1976) years. The X-745-E yard, which had been a compacted gravel area, was reconstructed during FY95-96 to a reinforced concrete yard. In FY96, 5,708 cylinders were relocated to meet new storage requirements.

In FY96, wall thickness UT measurements were made on $10 \%$ of the cylinders that were relocated. The sampled cylinders were selected using a random number generator. The $10 \%$ evaluation criterion was required according to the Consent Decree with the Ohio Environmental Protection Agency. These cylinders, as well as other cylinders with handling or storage damage, were evaluated using the automated scanner P-scan system and hand-held measurements for head/skirt areas. During subsequent years many of these same cylinders were remeasured. Many of the measurements are thus duplicates-measurements made on the same cylinders during multiple FY's. In these cases, only the most recent measurements were used in the corrosion modeling, which, because of statistical independence requirements, assumes that all UT measurements on any given cylinder were made at essentially the same time.

E-yard cylinders at Portsmouth are, on the whole, in worse shape than C-yard cylinders. However, because most of the Portsmouth UT data is for E-yard cylinders, all (E and C-yard) thin-wall, top-row cylinders are treated as one group, and all thin-wall, bottom-row cylinders are treated as one group in the regression modeling (Section 4). The bottom-row cylinders thus represent the worst-case (of two for thin-wall cylinders) at Portsmouth. There are 8,765 unpainted, age-known, bottom-row cylinders, and 8,500 unpainted, age-known, top-row cylinders. P-scan and manual UT measurement data for these populations are inventoried in Table 2c.

Thick-Wall Cylinders. There are 2,023 thick-wall cylinders (nominal wall thickness 625 mils) at the three sites: 1,264 at Portsmouth, 366 at PGDP, and 393 at ETTP. No thick-wall cylinders are designated in the CID as painted. The ages of two cylinders at ETTP and 20 at PGDP are unknown; otherwise the ages are known. The age ranges for thick-wall cylinders are 23-52 years at PORTS, 10-52 years at PGDP, and 4152 years at ETTP. Of the thick-wall cylinders that are of known age (and unpainted), 951 are top-row cylinders and 1,051 are bottom-row cylinders. The bottom-row cylinders are the "worst case," though with thicker walls, corrosion problems are less of a risk for these cylinders than for thin-wall cylinders.

Virtually all UT measurements of thick-wall cylinders have been made at Portsmouth (see Table 2c). During FY96, 115 thick-wall cylinders were measured with the P-scanner as part of cylinder relocation efforts. Those cylinders were selected because of ease of accessibility (Lykins and Pawel 1997). Because of limitations on the total number of measurements, this P-scan data is combined with later manual UT measurements for thick-wall cylinders.

Thin-Wall Skirted Cylinders. The CID lists 5,453 thin-wall cylinders as having skirts, 3,299 at PORTS, 1,493 at ETTP, and 661 at PGDP. Of the 5,453, 4,768 are unpainted and of known age: all of the cylinders at Portsmouth, 811 at ETTP, and 658 at PGDP. The ages of these skirted cylinders range from 17 to 47 years. Of the 4,768 cylinders that are unpainted and of known age, 2,484 are stored in bottom rows and 2,284 are in top rows. In the corrosion model, these cylinders are divided only by the top/bottom status, with the bottom cylinders assumed to represent the worst case.

Because of a combination of extended times of wetness and differential aeration (Lykins and Pawel 1997), there is a concern about the possibility of accelerated corrosion in the head/skirt interface crevice. In order to comply with the Ohio EPA Director's Findings and Orders for Portsmouth cylinder movements performed in FY96, wall thickness measurements at Portsmouth were made during FY97 at the head/skirt interface of 230 thin-wall cylinders. Many of these cylinders were also measured again during subsequent years. The head/skirt UT data for thin-wall cylinders is inventoried in Table 2c. 
Thick-Wall Skirted Cylinders. All of the 2,023 thick-wall cylinders discussed above are skirted. As with the thin-wall skirted cylinders, concerns about corrosion vulnerability at the head/skirt interface also apply, though to a lesser extent, to thick-wall cylinders. As part of the compliance with the Ohio EPA Director's Findings and Orders for cylinder movements performed in FY96, wall thickness measurements at Portsmouth were made during FY97 at the head/skirt interface of 114 thick-wall cylinders. Some of these cylinders were also measured again during subsequent years. The head/skirt UT data for thick-wall cylinders is inventoried in Table $2 \mathrm{c}$.

1/2" thick (30" diameter) Cylinders. There are 1,825 30", 1/2" nominal thickness, cylinders at Paducah, 412 at Portsmouth, and 726 at ETTP. All of these cylinders are unpainted and of known age, except for 89 cylinders at ETTP and 2 at Portsmouth. Precise historical information is not available on each cylinder, but it is known that nearly all of these cylinders were manufactured around 1954. Except for thirteen 30" cylinders measured at Portsmouth in FY96 and FY98, all 30" cylinder thickness data is for Paducah cylinders. This data is inventoried in Table $2 b$. 


\section{DATA ANALYSIS}

This section is about regression modeling with the data discussed in Section 3 and the indirect and direct corrosion models considered in Section 2. The indirect and direct models are based on a cylinder classification system with seventeen subpopulations. The same classification is used for both the direct and indirect models, and the two models may thus be compared for each subpopulation. The subpopulations are listed in Table 3. The classification is the same as the classification in Section 3 (Tables 2a-d), with a few modifications. The modifications are explained in Section 4.1 in conjunction with the discussion of analyses based on the indirect model, which are considered first. The direct model is considered in Section 4.2 .

The choice of cylinder subpopulations should strike a balance between model and statistical precision. Combining cylinder groups increases statistical precision, and, as long the groups do not differ too much, is often beneficial when there is little data. When there is ample data, that benefit is only minimal and it is usually better to increase model precision by using more groups. The direct approach, which incorporates all of the data into a single analysis, can more easily accommodate additional subpopulations than the indirect approach, which requires a separate regression for each subpopulation.

Table 3. The Seventeen Subpopulation Classification System Used in the Regression Modeling

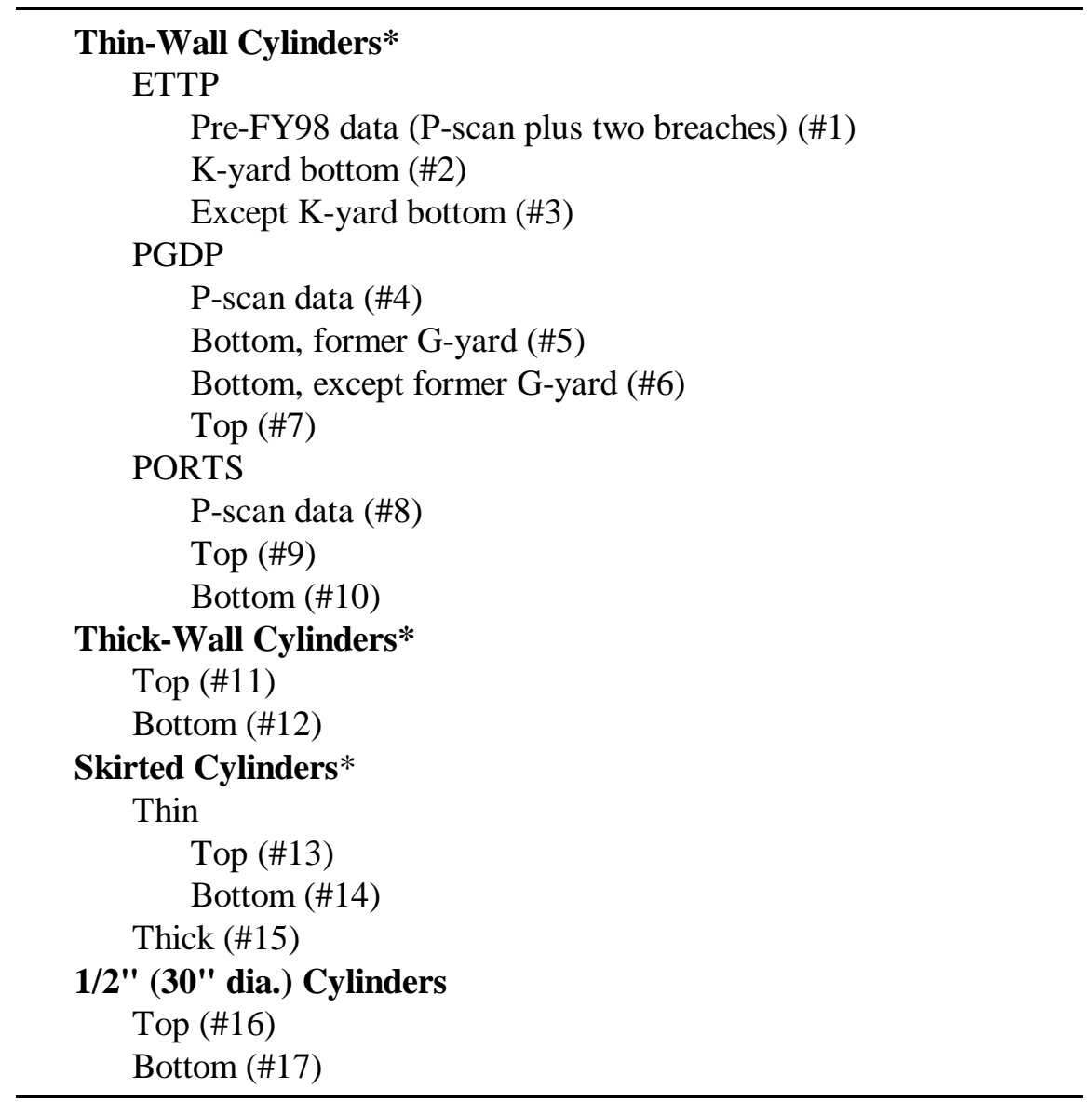

*Main body measurements and head/skirt interface measurements may be on the same cylinders (i.e., the populations overlap). 
Figures 1-17 in Appendix A are scatter plots of maximum pit depth estimates, for each of the seventeen cylinder groups. Figures 18-34 in Appendix A are scatter plots of the minimum thickness measurements for each cylinder group. Figures 1-34 also illustrate the indirect or direct model fitted to the maximum pit depth or minimum thickness data. Figures 18-34 also contain charts of the distributions of cylinder ages in underlying subpopulations from which the data is sampled. Projections based on both the direct and indirect fitted regression models are discussed in Section 5, and the direct and indirect models are compared in Section 6.

Several of Figures 1-34 contain points that are highlighted. These points, which are statistical outliers, are discussed in Section 4.3. Reanalyses with both the direct and indirect model are performed without the outliers, and the results are the basis for an alternative set of projections discussed in Section 5.

\subsection{Indirect Model Regressions}

Table 4 summarizes the indirect model results for the seventeen cylinder populations. In addition to the numerical results discussed in this section, the table also shows sample and population sizes, and initial thickness estimates.

ETTP Thin-wall, P-scan data, plus the two FY92 breaches (subpopulation \#1). The two breached cylinders discovered in FY02 are included with this pre-FY98 data, which otherwise is all from P-scans. The primary, fitted-slope, indirect model for the pit depths fails because the fitted slope estimate exceeds 1 , which suggest accelerating corrosion. Therefore, in accordance with the indirect modeling approach, the model with slope $=1$ is used instead. For that model, the intercept estimate turns out to be .532 . As will be seen in Section 5, with or without the breaches, this data leads to higher corrosion estimates and projections than the corresponding manual UT data. Because of the greater quantity and quality of manual UT (postFY98) data, and because the P-scan method itself was deprecated, these P-scan results are deprecated in favor of the following.

Measurements made by manual UT in FY98 are significantly different (e.g., medians are different; see Lyon 1998, Appendix D) from the measurements made before that with P-scan equipment. The manually collected data shows in general both a lower amount of wall loss and larger minimum thickness (mean: -49.8; standard error: 8.8; significance level of difference: .002). This is consistent with the results obtained in Schmidt et al (1996), where it was found that the P-scan measurements under-predicted minimum wall thickness. Schmidt et al found that, generally, the P-scan method resulted in underestimates of minimum wall thickness by an average of 10-20 mils. However, rather than manipulating the P-scan or manual UT data, in this report, the more recent data manual UT data will simply supersede the old.

ETTP Thin-wall, K-1066-K bottom (subpopulation \#2). This population is treated separately from the other ETTP populations, because a large portion of these cylinders were in ground contact for extended periods while they were in a previous yard (K-1066-G yard), and because, in K-yard as in other yards, cylinders are more likely to corrode in bottom rows than top rows. Thus this subpopulation is believed to approximate a worst case at ETTP. However, according to the indirect-model analysis, corrosion for this subpopulation is actually less severe than for the P-scan group above. The best fitted-slope estimate is negative, and so (in accordance with the indirect modeling approach described in Section 2) the slope $=1$ model is used instead. The fitted intercept for the slope $=1$ pit depth model is $-.564 \pm .057$ ( \pm one standard error). Thus, the fitted model is $\log (P($ age $))=-.564+\log ($ age $)$, or $P($ age $)=.569 \times$ age. The regression standard deviation (root mean squared error) is .796 log-scale mils (Table 4), which shows the substantial variability of the log-scale data about the fitted regression line $( \pm 2$ regression standard deviations is a $95 \%$ prediction range for an observation about the regression line). 
Table 4. Summary of Indirect-Model Populations and Modeling Assumptions

\begin{tabular}{|c|c|c|c|c|c|c|c|c|c|c|c|}
\hline $\begin{array}{l}\text { Cylinder } \\
\text { Grouping }\end{array}$ & Population & Model & $\begin{array}{c}\text { Sample } \\
\text { Size }\end{array}$ & $\begin{array}{c}\text { Inter- } \\
\text { cept }\end{array}$ & Slope & $\begin{array}{l}\text { Std. } \\
\text { Dev. }\end{array}$ & $\begin{array}{c}\text { Initial } \\
\text { Thick- } \\
\text { ness } \\
\text { Sample } \\
\text { Size }\end{array}$ & $\begin{array}{l}\text { Initial } \\
\text { Mean }\end{array}$ & $\begin{array}{c}\text { Initial } \\
\text { Std }\end{array}$ & $\begin{array}{c}\text { Initial } \\
\text { Thick- } \\
\text { ness } \\
\text { Inter- } \\
\text { val }\end{array}$ & $\begin{array}{c}\text { Total } \\
\text { in } \\
\text { Popu- } \\
\text { lation }\end{array}$ \\
\hline \multirow[t]{10}{*}{ Thin-Walled } & ETTP thin (P-scan data) & Slope $=1$ & 117 & 0.532 & 1.000 & 0.456 & 117 & 315.1 & 9.8 & {$[302.5,340]$} & 4,037 \\
\hline & $\begin{array}{r}\text { ETTP thin, } \begin{array}{r}\mathrm{K}-\mathrm{yard} \\
\text { bottom }\end{array}\end{array}$ & Slope $=1$ & 192 & -.564 & 1.000 & 0.796 & 192 & 332.2 & 11.7 & {$[302.5,379]$} & 1,167 \\
\hline & $\begin{array}{l}\text { ETTP thin, except } \\
\text { K-yard bottom }\end{array}$ & Slope $=1$ & 138 & -.688 & 1.000 & 0.931 & 138 & 331.2 & 9.7 & {$[302.5,368]$} & 2,870 \\
\hline & $\begin{array}{r}\text { PGDP thin (P-scan } \\
\text { data })\end{array}$ & Slope Fit & 350 & 1.507 & 0.715 & 0.390 & 350 & 333.8 & 9.3 & {$[302.5,363]$} & 32,231 \\
\hline & $\begin{array}{l}\text { PGDP thin bottom, } \\
\text { former G-yard }\end{array}$ & Slope Fit & 130 & 0.981 & 0.447 & 0.778 & 130 & 323.4 & 13.8 & {$[302.5,350]$} & 1,983 \\
\hline & $\begin{array}{l}\text { PGDP thin btm, excpt } \\
\text { fmr G-yard }\end{array}$ & Slope Fit & 325 & 0.767 & 0.499 & 0.829 & 325 & 328.8 & 13.2 & {$[302.5,395]$} & 15,347 \\
\hline & PGDP thin top & Slope Fit & 214 & 1.481 & 0.239 & 0.677 & 214 & 329.5 & 12.2 & {$[302.5,376]$} & 14,901 \\
\hline & $\begin{array}{r}\text { PORTS thin (P-scan } \\
\text { data })\end{array}$ & Slope Fit & 556 & 2.565 & 0.395 & 0.273 & 556 & 332.6 & 13.5 & {$[302.5,378]$} & 17,269 \\
\hline & PORTS thin top & Slope Fit & 218 & 2.398 & 0.240 & 0.455 & 218 & 355.7 & 12.3 & {$[302.5,389]$} & 8,501 \\
\hline & PORTS thin bottom & Slope Fit & 222 & 2.198 & 0.296 & 0.453 & 222 & 355.8 & 13.4 & {$[302.5,430]$} & 8,768 \\
\hline
\end{tabular}


Table 4-cont'd. Summary of Indirect-Model Populations and Modeling Assumptions

\begin{tabular}{|c|c|c|c|c|c|c|c|c|c|c|c|}
\hline $\begin{array}{l}\text { Cylinder } \\
\text { Grouping }\end{array}$ & Population & Model & $\begin{array}{c}\text { Sample } \\
\text { Size }\end{array}$ & $\begin{array}{c}\text { Inter- } \\
\text { cept }\end{array}$ & Slope & $\begin{array}{l}\text { Std. } \\
\text { Dev. }\end{array}$ & $\begin{array}{c}\text { Initial } \\
\text { Thick- } \\
\text { ness } \\
\text { Sample } \\
\text { Size }\end{array}$ & $\begin{array}{l}\text { Initial } \\
\text { Mean }\end{array}$ & $\begin{array}{c}\text { Initial } \\
\text { Std }\end{array}$ & $\begin{array}{c}\text { Initial } \\
\text { Thick- } \\
\text { ness } \\
\text { Inter- } \\
\text { val }\end{array}$ & $\begin{array}{c}\text { Total } \\
\text { in } \\
\text { Popu- } \\
\text { lation }\end{array}$ \\
\hline \multirow[t]{2}{*}{ Thick-Walled } & $\begin{array}{r}\text { ETTP/PGDP/PORTS } \\
\text { thick top }\end{array}$ & Slope Fit & 485 & 3.419 & 0.046 & 0.445 & 69 & 650.7 & 29.0 & {$[615,749]$} & 962 \\
\hline & $\begin{array}{r}\text { ETTP/PGDP/PORTS } \\
\text { thick bottom }\end{array}$ & Slope Fit & 488 & 3.521 & 0.045 & 0.513 & 76 & 651.9 & 29.6 & {$[615,727]$} & 1,062 \\
\hline \multirow[t]{3}{*}{ Skirted } & $\begin{array}{r}\text { ETTP/PGDP/PORTS } \\
\text { thin skirted top }\end{array}$ & Slope $=1$ & 164 & -.758 & 1.000 & 0.665 & 164 & 355.1 & 18.2 & {$[302.5,435]$} & 2,285 \\
\hline & $\begin{array}{l}\text { ETTP/PGDP/PORTS } \\
\text { thin skirted btm }\end{array}$ & Slope $=1$ & 176 & -.744 & 1.000 & 0.876 & 176 & 352.3 & 15.1 & {$[302.5,388]$} & 2,487 \\
\hline & $\begin{array}{r}\text { ETTP/PGDP/PORTS } \\
\text { thick skirted }\end{array}$ & Slope $=1$ & 137 & -.222 & 1.000 & 0.792 & 137 & 774.1 & 23.0 & {$[615,849]$} & 2,024 \\
\hline \multirow[t]{2}{*}{ 1/2" (30" dia.) } & $\begin{array}{l}\text { ETTP/PGDP/PORTS } \\
1 / 2 " \text { (30" dia.) top }\end{array}$ & Slope $=1$ & 123 & -.177 & 1.000 & 0.843 & 123 & 522.9 & 29.7 & {$[490,595]$} & 1,317 \\
\hline & $\begin{array}{l}\text { ETTP/PGDP/PORTS } \\
1 / 2 " \text { (30" dia.) btm }\end{array}$ & Slope $=1$ & 110 & 0.003 & 1.000 & 0.815 & 110 & 521.6 & 25.6 & {$[490,587]$} & 1,646 \\
\hline
\end{tabular}




\section{Table 5. Comparison of Estimated Minimum Point Wall Thickness Using Different Measurement Methods for Cylinders at K-1066-K Yard}

\begin{tabular}{cccc}
\hline Cylinder & $\begin{array}{c}\text { Estimated Minimum } \\
\text { Wall Thickness (mils) } \\
\text { by P-scanning (FY94) }\end{array}$ & $\begin{array}{c}\text { Estimated Minimum } \\
\text { Wall Thickness (mils) by } \\
\text { Manual UT (FY98) }\end{array}$ & $\begin{array}{c}\text { Difference } \\
\text { (mils) }\end{array}$ \\
\hline 5280 & 230 & 311 & -81 \\
6294 & 260 & 304 & -44 \\
6622 & 250 & 304 & -54 \\
7340 & 140 & 200 & -60 \\
7486 & 205 & 220 & -15 \\
14375 & 280 & 326 & -46 \\
\hline
\end{tabular}

ETTP Thin-wall, except K-1066-K bottom (subpopulation \#3). This cylinder data represents the complement of the ETTP thin-wall manual UT data not included in the worst-case group. Again, however, the fitted slope model fails because the fitted-slope estimate is negative. Therefore the slope $=1$ model is used instead. The intercept estimate is $-.688 \pm .079$. Thus, the best fitted model is $\log (\mathrm{P}(\mathrm{age}))=-.688+$ $\log ($ age $)$, or $\mathrm{P}($ age $)=.503 \times$ age. The regression standard deviation is .931 log-scale mils.

PGDP thin-wall, P-scan data (subpopulation \#4). For the PGDP P-scan data, the fitted-slope model fits. The regression intercept and slope estimates are 1.507 and .715 , so that the fitted model is $\log (P($ age $))=$ $1.507+.715 \log ($ age $)$, or $P($ age $)=.4 .51 \times(\text { age })^{0.715}$. Like the P-scan data for the other sites, this P-scan data paints a somewhat more pessimistic story than the corresponding manual UT data. This can be seen by examining either regression coefficients (see below) or the numbers of cylinders projected to fail various thickness criteria (Table 9A). As with the P-scan data for the other sites, this data is deprecated in favor of more recent manual UT results.

PGDP thin-wall bottom, former G-yard (subpopulation \#5). The former C-745-G yard represents the worst conditions at the PGDP site. Many of the cylinders stored there were in ground contact for extended periods. Unlike K-1066-yard bottom cylinders at ETTP, there is a wide range of ages for these cylinders (compare histograms in Figures 19 and 21). The fitted-slope model fits for these cylinders, and the fitted model is $\log (P($ age $))=.981+.447 \log ($ age $)$ or $P($ age $)=2.67 \times(\text { age })^{0.447}$. The regression standard deviation is $.778 \log$-scale mils. Comparisons with the fits for the other PGDP thin-wall groups (except for the P-scan group) are consistent with the assumption that the former G-yard bottom cylinders represent the worst case (see also projections in Table 9A).

PGDP thin-wall bottom, except former G-Yard (subpopulation \#6). Bottom-row cylinders other than those in former G-yard were not in ground contact for extended periods, with the possible exception of some of the F-yard cylinders. Former F yard is considered to be the PGDP yard with the next worst conditions after $\mathrm{G}$ yard. However, on the basis of all of the thin-wall cylinder data collected through FY02, there does not appear to be a reason to classify former F-yard bottom cylinders separately from other bottom-row cylinders (other than from G-yard). The overall mean for the F-yard bottom row cylinders is $306.8 \pm 2.3$ (one standard error), whereas the overall mean for the other (non-G-yard) bottom row cylinders is $305.4 \pm 1.1$. (On the other hand, the overall mean for G-yard bottom cylinders is $284.0 \pm 2.3$.) 
Therefore, as in previous versions of this report, the G/non-G yard division is retained, and no F/non-F yard divisions are made for Paducah cylinders.

The fitted-slope power-law model fits for these cylinders. The regression intercept and slope estimates are .708 and .519 , so that the fitted pit depth model is $\log (P($ age $))=.708+.519 \log ($ age $)$, or $P($ age $)=2.03 \times$ (age $)^{0.519}$ mils. The regression standard deviation is .821 log-scale mils.

PGDP thin-wall top (subpopulation \#7). Few of the cylinders in the top rows of these yards were ever in extended ground contact. The power law model that best fits the pit depth data for these cylinders is $\log (P($ age $))=1.546+.216 \log ($ age $)$, or $P($ age $)=4.49 \times(\text { age })^{0.216}$ mils. The regression standard deviation is .687 log-scale mils.

Portsmouth thin-wall cylinders, P-scan data (subpopulation \#8). For the 556 cylinders P-scanned at Portsmouth, the fitted-slope power-law model fits. The regression intercept and slope estimates are 2.565 and .395 , so that the fitted model is $\log (P($ age $))=2.565+.395 \times \log ($ age $)$, or $P($ age $)=13.0 \times(\text { age })^{0.395}$ mils. The regression standard deviation is .273 log-scale mils. As with P-scan data for cylinders at ETTP and PGDP, these results suggest greater corrosion rates than for the more recent manual UT scans from the same cylinder population (see below). The P-scan results are deprecated in favor of the manual UT data.

Portsmouth thin-wall cylinders, top (subpopulation \#9). The power law model that best fits the pit depth data for the bottom row cylinders is $\log (P($ age $))=2.398+.240 \log ($ age $)$, or $P($ age $)=11.0 \times$ $(\text { age })^{0.240}$. The regression standard deviation is .455 log-scale mils. There seems to be little difference between the results for these top-row cylinders and the Portsmouth bottom-row thin-wall cylinders (see below for subpopulation \#10).

Portsmouth thin-wall cylinders, bottom (subpopulation \#10). The power law model that best fits the pit depth data for the bottom row cylinders is $\log (P($ age $))=2.198+.296 \log ($ age $)$, or $P($ age $)=9.0 \times(\text { age })^{0.296}$. The regression standard deviation is $.453 \mathrm{log}$-scale mils. Although these are bottom-row cylinders, few of them have ever been in extended ground contact. Few, in fact, from either the bottom or the top rows have ever been in extended ground contact, and there seems to be little difference between the results for the Portsmouth top and bottom rows. Nevertheless, there are plenty of UT measurements for both the top and bottom classes, and so there would be little if any improvement to the classification in Table 3 if the top and bottom Portsmouth thin-wall groups were combined.

Thick-Wall cylinders, Top Rows (subpopulation \#11). Since FY96, UT measurements have been made for 73 unique, thick-wall, top-row cylinders. Some of the cylinders were measured more than once, and in such cases only the latest measurement is used in the regression analyses. The age range of these cylinders is narrow: 49-52 years. Because of the narrow age range, and because it is expected that the distribution of pit depths for thin-wall cylinders at Portsmouth is similar to the distribution for the thick-wall cylinders, the maximum pit depth data for thin-wall cylinders in the top rows at Portsmouth was added to the thick-wall data set, and a model for pit depths was then derived. Of course only the thick-wall cylinders are used for estimating initial thicknesses. ${ }^{8}$ This indirect model is assumed to apply to top-row, thick-wall cylinders at all yards, though essentially all of the thick-wall cylinder measurements have been made for cylinders at Portsmouth. In addition to 615 top-row, thick-wall cylinders at Portsmouth, there are 132 at PGDP and 204 at ETTP. With the combined thick and thin-wall data, the power law model that best fits the pit depth data for the top row cylinders is $\log (P($ age $))=3.419+.046 \log ($ age $)$, or $P($ age $)=30.5 \times(\text { age })^{0.046}$. The regression standard deviation is $.445 \log$-scale mils.

${ }^{8}$ In the direct-model regressions, thin-wall cylinders are not included at all in the thick-wall groups. 
Thick-Wall cylinders, Bottom Rows (subpopulation \#12). Since FY96, UT measurements have been made for 72 unique, thick-wall, top-row cylinders. Some of the cylinders were measured more than once; in such cases only the latest measurement is used in the regression analyses. The age range of these bottom-row cylinders is the same as for the top-row thick-wall cylinders: 49-52 years. Because of the narrow age range, and because it is expected that the distribution of pit depths for thin-wall cylinders at Portsmouth is similar to the distribution for the thick-wall cylinders, the maximum pit depth data for thinwall cylinders in the bottom rows at Portsmouth was added to the data set, and a model for pit depths was then derived. As for the top-row thick-wall cylinders, only the thick-wall cylinders are used for estimating initial thicknesses. This indirect model is assumed to apply to bottom-row, thick-wall cylinders at all yards, though essentially all of the thick-wall cylinder measurements have been made for cylinders at Portsmouth. In addition to 650 bottom-row, thick-wall cylinders at Portsmouth, there are 214 at PGDP and 187 at ETTP. With the combined thick and thin-wall data set, the power law model that best fits the pit depth data for the bottom row cylinders is $\log (P($ age $))=3.521+.045 \log ($ age $)$, or $P($ age $)=33.8 \times$ (age $)^{0.045}$. The regression standard deviation is .513 log-scale mils. As with the thin-wall cylinders at Portsmouth, the top and bottom-row thick-wall populations, combined with the thin-wall cylinders, are essentially the same.

Thin-Wall Skirted Cylinders, Top (subpopulation \#13). The wall thickness at the head/skirt interface was measured for 164 top-row thin-wall skirted cylinders at Portsmouth since FY97. Figure 13 shows that this data suggests a negative power-law slope, but there are no obvious outliers, and there does not seem to be a way to decide which of the data should be rejected and which should be kept. (The problem is also because of the narrow range of ages, and because the constraint $\mathrm{P}(0)=0$ cannot easily be formulated on the $\log$ scale.) Therefore the slope-set-to-one model is used. The model with slope $=1$ that best fits the pit depth data for the top row skirted cylinders is $\log (P($ age $))=-.758+\log ($ age $)$, or $P($ age $)=.469 \times$ age. The regression standard deviation is $.665 \log$-scale mils.

Thin-Wall Skirted Cylinders, Bottom (subpopulation \#14). The wall thickness at the head/skirt interface was measured for 176 bottom-row thin-wall skirted cylinders at Portsmouth since FY97. Figure 14 shows that, as with data for the thin-wall skirted top-row cylinders, this data also suggests a negative power-law slope. Therefore the slope-set-to-one model is used. The model with slope $=1$ that best fits the pit depth data for the top row skirted cylinders is $\log (P($ age $))=-.744+\log ($ age $)$, or $P($ age $)=.475 \times$ age. The regression standard deviation is .876 log-scale mils. As with other Portsmouth subpopulations, there appears to be little difference between the top and bottom-row cylinders.

Thick-Wall Skirted Cylinders (subpopulation \#15). The wall thickness at the head/skirt interface was measured for 137 thick-wall skirted cylinders at Portsmouth since FY97. The top and bottom cylinders were combined for this group, because there is not enough new thick-wall head/skirt data to warrant separate top and bottom classes. As with the thin-wall head/skirt data, the thick-wall head/skirt data suggests a negative power-law slope. Therefore, the slope-set-to-one model is used. The pit depth model with slope $=1$ that best fits the pit depth data for the top row skirted cylinders is $\log (P($ age $))=-.222+$ $\log ($ age $)$, or $P($ age $)=.801 \times$ age. The regression standard deviation is .792 log-scale mils.

1/2" (30" diameter) top-row cylinders (subpopulation \#16). Table 6 shows summary statistics for $1 / 2$ " cylinders. In previous editions of this report, top and bottom 1/2" (30" diameter) cylinders were modeled together as one group, and in fact the data still shows much greater year-to-year differences than top-tobottom differences (Table 6). Nevertheless, with 25 additional 30" cylinder UT scans performed in FY02, there are now over 100 thickness measurements available each for the top and bottom cylinders, and there is also at least a suggestion that the bottom-row conditions are worse- an analysis of variance shows that the maximum pit depths are significantly higher for the bottom-row cylinders $(\mathrm{p}=.04)$. Therefore the $1 / 2 "$ cylinders are classified by top/bottom status in this report. 
The $1 / 2$ " cylinder, top-row thickness data (Figure 16) suggests a negative power-law slope. Therefore, the slope-set-to-one model is used. The pit depth model with slope $=1$ that best fits the pit depth data for the top row $1 / 2$ " cylinders is $\log (P($ age $))=-.177+\log ($ age $)$, or $P($ age $)=.843 \times$ age. The regression standard deviation is .843 log-scale mils.

1/2" (30" diameter) bottom-row cylinders (subpopulation \#17). The 1/2" cylinder, bottom-row thickness data (Figure 17) suggests a negative power-law slope. Therefore, the slope-set-to-one model is used. The pit depth model with slope $=1$ that best fits the pit depth data for the bottom row $1 / 2$ " cylinders is $\log (P($ age $))=.003+\log ($ age $)$, or $P($ age $)=1.00 \times$ age. The regression standard deviation is $.815 \log$-scale mils.

\section{Table 6. 1/2" (30" dia.) Cylinder Minimum Thicknesses and Estimated Pit Depths by Top/Bottom Status and FY}

\begin{tabular}{ccccc}
\hline FY & $\begin{array}{c}\text { Top/Bottom } \\
\text { Status }\end{array}$ & $\mathbf{N}^{*}$ & $\begin{array}{c}\text { Mean Minimum } \\
\text { Thickness } \\
\text { (Standard Error) }\end{array}$ & $\begin{array}{c}\text { Mean Log Maximum } \\
\text { Estimated Pit Depth } \\
\text { (Standard Error) }\end{array}$ \\
\hline \multirow{2}{*}{99} & Top & 51 & $440.9(11.2)$ & $3.92(.10)$ \\
& Bottom & 46 & $438.8(9.0)$ & $4.00(.10)$ \\
601 & Top & 61 & $487.7(8.8)$ & $3.43(.11)$ \\
& Bottom & 38 & $472.6(12.2)$ & $3.88(.14)$ \\
602 & Top & 11 & $455.6(23.0)$ & $3.63(.28)$ \\
& Bottom & 14 & $444.7(19.6)$ & $3.52(.32)$ \\
\hline
\end{tabular}

*Cell counts differ slightly from 2001 report because of FY02 replicate measurements.

\subsection{Direct Model Regressions}

Without setting the power-law slope to one, the indirect corrosion model would fail for eight of the seventeen cylinder subpopulations. Some of the failures may be due to the statistically variable and sometimes inconsistent nature of the maximum pit depth estimates computed from maximum thickness measurements as a proxy for initial thickness. The reason for the failures may also be for physical reasons, for example because of changed maintenance and storage conditions, or because the power-law model is based on corrosion physics that may apply to small objects such as metal coupons under ideal conditions, not necessarily to thermally massive storage cylinders that are abraded, nicked, and cut during one or more use cycles.

Whether for theoretical reasons or because of practical data limitations, it seems appropriate to try an approach that (1) does not require the estimation of pit-depth maxima, and (2) smooths out data anomalies by imposing more structure than the structure in the indirect approach's seventeen separately-fitted regressions. The direct model does not require pit-depth estimation and does provide more structure.

Original Thickness Estimates. The direct model requires estimates of the initial cylinder thickness. For initial thickness estimates, design-sheet specifications are a good starting point, but, as Table 7 suggests, design-sheet specifications can be refined. For each of the seventeen cylinder groups developed in Section 
4.1, Table 7 contains $97.5 \%$ one-side lower and upper confidence limits (which together compose a 95\% confidence interval) for the mean maximum thickness. The confidence limits are computed from wall maximum thickness measurements for each cylinder group. The table also contains the nominal lower and upper design limits, from the design sheets. In the final column, the table contains an original thickness estimate, which combines the nominal and confidence limits. These original thickness estimates are used in the direct-model regressions.

The original thickness estimates in Table 7 are computed as follows. As can be seen from the table, except for the thick, skirted group and the $1 / 2$ " cylinders, the confidence limit ranges are not far from the nominal ranges and in most cases overlap them. For the thick, skirted group and the $1 / 2$ " groups, the $97.5 \% \mathrm{LCL}$ was taken as the original thickness estimate, which is the point in the $95 \%$ confidence range closest to the nominal thickness range. For the other groups, except for the thin, skirted groups, the confidence intervals and design ranges actually do overlap. For these other groups, when the confidence and nominal ranges overlap, the original thickness estimate was taken as the midpoint of the range of overlap. When the confidence and nominal ranges do not overlap, the nominal range endpoint nearest to the confidence interval was taken as the original thickness estimate. Thus, except for the thick, skirted group and the $1 / 2$ " cylinders, the original thickness estimate is defined as follows:

If Nominal Upper $<$ LCL, then Original Estimate = Nominal Upper;

Otherwise, if UCL < Nominal Lower, then Original Estimate = Nominal Lower;

Otherwise, Original Estimate $=[\min ($ UCL, Nominal Upper $)+\max (L C L$, Nominal Lower $)] / 2$.

Because a nominal range endpoint is used when the confidence and nominal ranges do not overlap, this algorithm for estimating the initial thickness favors the nominal specification. The rationale for preferring the nominal specification is that (1) if the original thickness of a cylinder was not uniform, then the maximum thickness (at any time) is likely to be a poor estimate of the original minimum thickness of the cylinder, and (2) the original estimates, so defined, seem to work well in the minimum thickness regression discussed below. For the thick, skirted group and the $1 / 2$ " cylinder groups, the discrepancy between the confidence limits and the nominal specification is so great that the nominal specification does not seem reasonable, and the confidence limit closest to the nominal range is used instead.

The original thickness estimates in Table 7 all exceed their corresponding nominal thicknesses $(312.5,625$, or 100 mils). The original thickness estimates were used in the direct model regression, as a predictor variable, the effect of which was adjusted in fitting the direct regression model (2.3). Thus a source of information for refining the original minimum thickness estimates is the UT minimum thickness data itself. Although the original thickness estimate in the direct regression model is assumed only to be an estimate (not the original thickness itself), the $\alpha \times$ (Original Thickness Estimate) term in the direct model (2.3) actually represents the mean thickness at one year of age (i.e., when $\log ($ age $)=0$ ). Thus we would expect $\alpha$ to be close to 1 and smaller than 1, though a departure from this is possible because of error in the original thickness estimates.

Weighting. Whether and how the fit of the regression model in one region of the space of predictor variables (e.g., subpopulation, original thickness, and age) can be used to make inferences (e.g., predictions) in another region depends on the statistical distribution of the underlying regression errors. For example, the variance of the distribution of minimum thickness measurements likely increases with cylinder age. This should be accounted for, because projections about minimum thicknesses at a target age in the future are based on measurements for cylinders at ages less than the target age. In the direct-model regression, the dependency of the variance on age is accounted for by weighting. 


\section{Table 7. Original Thickness Estimates}

\begin{tabular}{|c|c|c|c|c|c|}
\hline Cylinder Group & $\begin{array}{c}97.5 \% \\
\text { LCL }\end{array}$ & $\begin{array}{c}97.5 \% \\
\text { UCL }\end{array}$ & $\begin{array}{l}\text { Nominal } \\
\text { Lower }\end{array}$ & $\begin{array}{c}\text { Nominal } \\
\text { Upper }\end{array}$ & $\begin{array}{c}\text { Orginal } \\
\text { Thickness } \\
\text { Estimate }\end{array}$ \\
\hline ETTP thin (P-scan data) & 313.3 & 316.9 & 302.5 & 345.5 & 315.1 \\
\hline ETTP thin, $\mathrm{K}-$ yard bottom & 330.5 & 333.8 & 302.5 & 345.5 & 332.2 \\
\hline ETTP thin, except $\mathrm{K}$-yard bottom & 329.6 & 332.8 & 302.5 & 345.5 & 331.2 \\
\hline PGDP thin (P-scan data) & 332.8 & 334.8 & 302.5 & 345.5 & 333.8 \\
\hline PGDP thin bottom, former G-yard & 321.0 & 325.8 & 302.5 & 345.5 & 323.4 \\
\hline PGDP thin btm, excpt fmr G-yard & 327.4 & 330.3 & 302.5 & 345.5 & 328.8 \\
\hline PGDP thin top & 327.8 & 331.1 & 302.5 & 345.5 & 329.5 \\
\hline PORTS thin (P-scan data) & 331.5 & 333.8 & 302.5 & 345.5 & 332.6 \\
\hline PORTS thin top & 354.1 & 357.4 & 302.5 & 345.5 & 345.5 \\
\hline PORTS thin bottom & 354.1 & 357.6 & 302.5 & 345.5 & 345.5 \\
\hline ETTP/PGDP/PORTS thick top & 643.7 & 657.7 & 615.0 & 655.0 & 649.4 \\
\hline ETTP/PGDP/PORTS thick bottom & 645.2 & 658.7 & 615.0 & 655.0 & 650.1 \\
\hline ETTP/PGDP/PORTS thin skirted top & 352.3 & 357.9 & 302.5 & 345.5 & 345.5 \\
\hline ETTP/PGDP/PORTS thin skirted btm & 350.1 & 354.6 & 302.5 & 345.5 & 345.5 \\
\hline ETTP/PGDP/PORTS thick skirted & 770.3 & 778.0 & 615.0 & 655.0 & 770.3 \\
\hline ETTP/PGDP/PORTS 1/2" (30" dia.) top & 517.6 & 528.2 & 343.8 & 468.8 & 517.6 \\
\hline ETTP/PGDP/PORTS 1/2" (30" dia.) btm & 516.8 & 526.4 & 343.8 & 468.8 & 516.8 \\
\hline
\end{tabular}

Three weighting strategies were investigated for the direct-model regressions: constant-variance (i.e., unweighted), variance-proportional-to-age, and variance-proportional-to-age-squared. The varianceproportional-to-age weighting was chosen on the basis of residual plots and judgment about data quality. (Regression residuals are the differences between the observed (dependent variable) values and their corresponding fitted values.) The regression weighted by age is easily implemented by dividing cylinder minimum thicknesses, original thickness estimates, and the log(age) terms by the square root of age. The ordinary, unweighted regression with the variables so transformed is equivalent to an age-weighted regression of the untransformed variables.

Direct-Model Regression Results. Table 8 shows the $\alpha$ and $\beta$ (group) parameter estimates for the directmodel regression with the variance-proportional-to-age weighting. The $\mathrm{R}^{2}$ value for the regression is $92.0 \%$. ${ }^{9}$ The $\alpha$ coefficient for the original thickness estimate, .97 , is in the range reasonably close to but less than 1. The $\beta$ (group) parameters should all be negative, because, according to the model $d M(t) / d t=$ $\beta$ (group) $/ t$. Although the direct model imposes no constraints on the $\beta$ (group) parameter estimates, all of the estimates do turn out to be negative, and there are no inconsistencies between model and data.

\footnotetext{
${ }^{9} \mathrm{R}^{2}$ is the squared multiple correlation coefficient or proportion of explained variance (see Draper and Smith, 1981). Although $\mathrm{R}^{2}$ statistics for the direct and indirect models are not directly comparable, for reference, the $\mathrm{R}^{2}$ values for the indirect models were less than .10 for all but five of the seventeen indirect model regressions and never exceeded .55 in the other five cases. These are actually the $\mathrm{R}^{2}$ for the unconstrained two-parameter power law model, even when the slope-set-to-one model is used instead. The $\mathrm{R}^{2}$ statistic for the slope-set-to-one model, which is an intercept-only model, is by definition always zero.
} 
Figures 18-34 show the fitted, direct-model, age-weighted regressions for the seventeen cylinder groups. In addition to plots of regression results, these figures also contain charts of the cylinder age distributions for the populations defined by the cylinder groups. These age distribution are for all cylinders in the population, not just for cylinders that were sampled. The age distribution charts show, in particular, the ages and counts for the oldest cylinders in each group, which, on average, are the cylinders at greatest risk. Estimates that are averages for entire groups can obscure risks for the oldest cylinders, for example, if the oldest cylinders are exceptions relative to the population in general (see for example Figures 23-26).

In addition to minimum thickness measurements, the regression plots in Figures 18-34 show the directmodel fitted regression curves and approximate 99\% lower confidence limits (LCLs) for minimum thicknesses for individual cylinders over the age ranges in the plots. The LCL curves are approximations:

$$
\text { Probability ( Actual Minimum Thickness at age } \mathrm{t} \geq \mathrm{LCL} \text { at } \mathrm{t} \text { ) } \approx .99
$$

for any particular age t. Two different sets of LCL curves are shown. One set is based on a large-sample approximation (Schmoyer 1992) that does not assume any particular underlying distribution (e.g., normal) for the regression errors. The other LCLs are the usual LCLs for individual predicted values, which are based on the assumption that regression errors have normal distributions. The normal-theory confidence limits are generally though not necessarily closer to the regression fitted curve than the large-sample limits. Both the normal-theory and large-sample LCLs suggest that although there are slight declines over time in average minimum wall thicknesses, there is considerable uncertainty about individual cylinders, and the uncertainty about individual cylinders increases as predictions extend farther ahead in time.

Figures 35 and 36 are plots of the regression residuals from the age-weighted regression. The plots can be used to help decide about whether the statistical distribution of the regression errors is normal or otherwise, whether the regression errors are approximately uniform (e.g., across ages), whether the varianceproportional-to-age weighting or some other weighting is appropriate. A uniform distribution in the weighted residuals is the objective of the weighting, because a uniform weighted error distribution is an assumption in statistical inferences (e.g., confidence bounds) based on the regression.

Figure 35 shows that for the regression weighted by age, the variance of the residuals is approximately uniform in age. There does appear to be a tendency for the weighted residuals to fan out with increasing age, however, though it occurs primarily for the $1 / 2$ " cylinders. The more severe, variance-proportional-toage-squared weighting was also tried here, but it did not have much effect on either the pattern of residuals or the wall-thickness projections based on the regression. As discussed in Section 4.1, there are problems with the $1 / 2$ " cylinder data. The average minimum thickness is significantly lower in FY01 than in FY99, for example. The $1 / 2$ "-thick cylinders could be modeled separately from the other cylinder groups, but one of the goals in the choice of the direct model is to encompass many cylinder groups with one model, so that anomalies in data anomalies such as the $1 / 2 "$-thick cylinder data can be smoothed out. Furthermore, no physical theories have yet been offered that would suggest that the $1 / 2$ "-thick cylinders, in particular, should be modeled separately from other cylinder groups. Therefore, the $1 / 2$ "-thick cylinders were modeled along with the other cylinders, using the variance-proportional-to-age weighting for the regression errors. Cylinders with unusually large negative residuals are considered as outliers in the discussion below.

Figure 36 shows the regression residuals in a normal probability plot. The figure shows that the distribution of residuals, and by extension the distribution of regression errors, is not normal, particularly for the lower (left) side of the distribution, where the residuals are smaller (more negative) than would be expected under normal theory. This suggests that the normal-theory LCLs for individual minimum thickness predictions are likely to be inaccurate, and that the large-sample confidence limits (which are lower than the normal-based limits) are probably better. Because the use of the normal-theory confidence limits is much more common in regression modeling, they were included in the figures as points of comparison for the large-sample limits. 


\section{Table 8. Direct-Model Parameter Estimates and Standard Errors}

\begin{tabular}{lrr}
\hline Parameter & Estimate & $\begin{array}{c}\text { Standard } \\
\text { Error }\end{array}$ \\
\hline Initial Thickness & 0.97 & 0.01 \\
ETTP thin (P-scan data) & -15.3 & 1.26 \\
ETTP thin, K-yard bottom & -6.20 & 1.17 \\
ETTP thin, except K-yard bottom & -6.06 & 1.24 \\
PGDP thin (P-scan data) & -12.8 & 1.21 \\
PGDP thin bottom, former G-yard & -2.02 & 1.26 \\
PGDP thin btm, excpt fmr G-yard & -1.85 & 1.20 \\
PGDP thin top & -0.42 & 1.25 \\
PORTS thin (P-scan data) & -12.6 & 1.21 \\
PORTS thin top & -2.41 & 1.25 \\
PORTS thin bottom & -2.56 & 1.24 \\
ETTP/PGDP/PORTS thick top & -6.91 & 2.17 \\
ETTP/PGDP/PORTS thick bottom & -7.04 & 2.15 \\
ETTP/PGDP/PORTS thin skirted top & -1.26 & 1.23 \\
ETTP/PGDP/PORTS thin skirted btm & -3.00 & 1.22 \\
ETTP/PGDP/PORTS thick skirted & -5.75 & 2.36 \\
ETTP/PGDP/PORTS 1/2" (30" dia.) top & -9.60 & 1.70 \\
ETTP/PGDP/PORTS 1/2" (30" dia.) btm & -11.3 & 1.72 \\
\hline
\end{tabular}

\subsection{Reanalyses after dropping outliers}

Data points plotted in Figures 1-34 were considered outliers if they met the following criteria:

For the indirect model (Figures 1-17): maximum pit depth > 160 mils

For the direct model (Figures 18-34): corresponding regression residual $<-20$ mils

Direct-model regression residuals are also plotted in Figure 35. Outliers (points failing the above criteria) are highlighted in yellow in figures showing them (e.g., Figures 6 or 20).

The maximum pit depth and minimum thickness data were re-analyzed with the outliers dropped using the direct and indirect models and the respective outlier criteria. In Sections 5 and 6 it is shown that projections based on the analyses without the outliers are in fact more consistent with experience than projections based on the analyses with the outliers included. Although being a statistical outlier does not automatically imply that anything is wrong with a data point, the more realistic conclusions based on the analyses without the outliers suggest that something may in fact be wrong with some of them. Therefore, cylinders whose thickness data fails the above criteria are good candidates for re-examination to either validate or correct their previous measurements. 


\section{WALL THICKNESS PROJECTIONS}

Using the fitted, indirect and direct models, projections were made of the number of cylinders with minimum wall thickness less than the following criteria:

1. 0 mils (i.e., a breach), which indicates a possible loss of contained material

2. 62.5 mils, below which ordinary safe handling and stacking is considered to be impaired

3. 250 mils for thin-wall cylinders and 500 mils for thick-wall cylinders, which represent applicable standards for off-site transport and contents transfer (based on ANSI 14.1 1995): .

For $1 / 2$ " (30" diameter) cylinders, there are no published criteria for minimum thicknesses. However, in addition to the zero (breach) and 62.5 mil criteria, 100 mils, the minimum thickness for regular hot feeding ${ }^{10}$ is also used for $1 / 2$ " cylinders.

These criteria are actually for on an area of wall thinning, as opposed to a point. Minimum thicknesses predictions calculated for this report are for a tiny area of only about 0.01 square inches, essentially a point. For thickness criteria greater than zero (breach), the calculations may therefore be conservative. For the breach criteria, consider the following from DNFSB (1995):

A breach in a cylinder allows the external atmosphere to react slowly with the $\mathrm{UF}_{6}$. The solid reaction product tends to plug the breach; however, the HF formed releases slowly, attacks the metal cylinder, and enlarges the breach over time. The hole diameter is estimated to increase at a rate of approximately one inch per year.

Therefore, because of the interaction of $\mathrm{UF}_{6}$ with atmospheric moisture and the substrate steel, the approximation of a small-area breach with a point breach is probably close. ${ }^{11}$

Cylinder Count Projections. Separate projections are made for the direct and indirect approaches. Table 9A shows numbers of cylinders projected on the basis of the indirect regression model to have minimum wall thickness below the various thickness criteria. Table 10A shows projections based on the direct model. These projections are computed using equation (2.1) with cylinder counts (from the CID) and either indirect or direct-model estimates of the probability $\operatorname{Prob}(M(t)<z)$ for the various ages $t$ and thickness criteria $z$. Thus the numbers in the tables are estimates of the numbers of cylinder expected to fail the various thickness criteria. Even if the estimates of $\operatorname{Prob}(M(t)<z)$ were exact, and the estimates of the numbers of cylinders expected to fail were thus also exact, the actual numbers of cylinders observed in practice to violate the various thickness criteria would still depart randomly from the estimates.

${ }^{10}$ S. J. Pawel, ORNL Corrosion Science \& Technology Group, personal communication.

${ }^{11}$ However, note also page 4 of the SRD (LMES 1997a): "Reaction deposits formed when UF $_{6}$ is exposed to the atmosphere in the presence of the mild steel containers have a self-sealing nature." 
Table 9A. Summary of Indirect-Model Projections for Target Years and Minimum Thickness Criteria

\begin{tabular}{|c|c|c|c|c|c|c|c|c|c|c|c|c|c|c|c|}
\hline \multirow[b]{2}{*}{ Cylinder Population } & \multirow[b]{2}{*}{$\begin{array}{c}\text { Pop. } \\
\text { Total } \\
\end{array}$} & \multirow[b]{2}{*}{ Model } & \multirow[b]{2}{*}{$\begin{array}{c}\text { Thick. } \\
\text { Spec } \\
\text { (mils) }\end{array}$} & \multicolumn{12}{|c|}{ Projected Number of Cylinders Below Thickness Criterion } \\
\hline & & & & $\begin{array}{l}20 \\
\text { Esti- } \\
\text { mate }\end{array}$ & $\begin{array}{l}03 \\
95 \% \\
\text { UCB } \\
\end{array}$ & $\begin{array}{r}20 \\
\text { Esti- } \\
\text { mate }\end{array}$ & $\begin{array}{l}005 \\
-95 \% \\
\text { UCB } \\
\end{array}$ & $\begin{array}{r}20 \\
\text { Esti- } \\
\text { mate } \\
\end{array}$ & $\begin{array}{l}10 \\
95 \% \\
\text { UCB }\end{array}$ & $\begin{array}{r}20 \\
\text { Esti- } \\
\text { mate }\end{array}$ & $\begin{array}{l}15 \\
95 \% \\
\text { UCB } \\
\end{array}$ & $\begin{array}{l}20 \\
\text { Esti- } \\
\text { mate }\end{array}$ & $\begin{array}{c}020 \\
95 \% \\
\text { UCB } \\
\end{array}$ & $\begin{array}{l}20 \\
\text { Esti- } \\
\text { mate }\end{array}$ & $\begin{array}{l}25 \\
95 \% \\
\text { UCB } \\
\end{array}$ \\
\hline \multirow[t]{3}{*}{ ETTP thin (P-scan data) } & 4,037 & Slope $=1$ & 250 & 1,726 & 1,983 & 1,895 & 2,169 & 2,293 & 2,607 & 2,644 & 2,971 & 2,939 & 3,259 & 3,179 & 3,477 \\
\hline & & & 62.5 & 8 & 36 & 10 & 45 & 20 & 75 & 36 & 116 & 59 & 169 & 92 & 233 \\
\hline & & & 0 & 2 & 12 & 2 & 15 & 5 & 27 & 9 & 44 & 17 & 67 & 27 & 97 \\
\hline \multirow[t]{3}{*}{ ETTP thin, K-yard bottom } & 1,167 & Slope $=1$ & 250 & 77 & 131 & 86 & $5 \quad 143$ & 110 & 172 & 135 & 202 & 161 & 232 & 188 & 262 \\
\hline & & & 62.5 & 1 & 6 & 2 & 7 & 3 & 10 & 4 & 13 & 5 & 17 & 7 & 21 \\
\hline & & & 0 & 1 & 3 & 1 & 4 & 1 & 5 & 2 & 7 & 2 & 9 & 3 & 12 \\
\hline \multirow[t]{3}{*}{ ETTP thin, except K-yard bottom } & 2,870 & Slope $=1$ & 250 & 144 & 281 & 162 & 2307 & 210 & 375 & 262 & 443 & 316 & 511 & 373 & 578 \\
\hline & & & 62.5 & 5 & 24 & 6 & 27 & 9 & 37 & 13 & 48 & 17 & 60 & 23 & 74 \\
\hline & & & 0 & 3 & 14 & 3 & 16 & 5 & 22 & 7 & 29 & 9 & 37 & 12 & 46 \\
\hline \multirow{3}{*}{ PGDP thin (P-scan data) } & 32,231 & Slope Fit & 250 & 2,509 & 4,181 & 2,928 & 4,789 & 4,186 & 6,481 & 5,745 & 8,345 & 7,555 & 10,314 & 9,526 & 12,386 \\
\hline & & & 62.5 & 1 & 8 & 1 & 10 & 3 & 20 & 6 & 36 & 10 & 60 & 18 & 96 \\
\hline & & & 0 & 0 & 1 & 0 & 2 & 0 & 4 & 1 & 7 & 2 & 13 & 3 & 22 \\
\hline \multirow[t]{3}{*}{ PGDP thin bottom, former G-yard } & 1,983 & Slope Fit & 250 & 32 & 119 & 34 & $4 \quad 127$ & 40 & 149 & 47 & 173 & 53 & 198 & 59 & 226 \\
\hline & & & 62.5 & 0 & 3 & 0 & 3 & 0 & 4 & 0 & 5 & 0 & 7 & 0 & 9 \\
\hline & & & 0 & 0 & 1 & 0 & 1 & 0 & 2 & 0 & 3 & 0 & 3 & 0 & 4 \\
\hline \multirow[t]{3}{*}{ PGDP thin btm, excpt fmr G-yard } & 15,347 & Slope Fit & 250 & 113 & 372 & 128 & 401 & 170 & 475 & 216 & 550 & 265 & 629 & 315 & 717 \\
\hline & & & 62.5 & 1 & 7 & 1 & 8 & 1 & 10 & 2 & 13 & 2 & 16 & 3 & 19 \\
\hline & & & 0 & 0 & 3 & 0 & 3 & 0 & 5 & 1 & 6 & 1 & 7 & 1 & 9 \\
\hline \multirow[t]{3}{*}{ PGDP thin top } & 14,901 & Slope Fit & 250 & 14 & 100 & 15 & $\quad 102$ & 19 & 109 & 23 & 117 & 26 & 126 & 30 & 139 \\
\hline & & & 62.5 & 0 & 0 & 0 & 0 & 0 & 0 & 0 & 0 & 0 & 0 & 0 & 1 \\
\hline & & & 0 & 0 & 0 & 0 & 0 & 0 & 0 & 0 & 0 & 0 & 0 & 0 & 0 \\
\hline
\end{tabular}


Table 9A-cont'd. Summary of Indirect-Model Projections for Target Years and Minimum Thickness Criteria

\begin{tabular}{|c|c|c|c|c|c|c|c|c|c|c|c|c|c|c|c|}
\hline \multirow[b]{2}{*}{ Cylinder Population } & \multirow[b]{2}{*}{$\begin{array}{c}\text { Pop. } \\
\text { Total } \\
\end{array}$} & \multirow[b]{2}{*}{ Model } & \multirow[b]{2}{*}{$\begin{array}{c}\text { Thick. } \\
\text { Spec } \\
\text { (mils) }\end{array}$} & \multicolumn{12}{|c|}{ Projected Number of Cylinders Below Thickness Criterion } \\
\hline & & & & $\begin{array}{r}20 \\
\text { Esti- } \\
\text { mate }\end{array}$ & $\begin{array}{l}03 \\
95 \% \\
\text { UCB } \\
\end{array}$ & $\begin{array}{r}20 \\
\text { Esti- } \\
\text { mate } \\
\end{array}$ & $\begin{array}{l}05 \\
95 \% \\
\text { UCB } \\
\end{array}$ & $\begin{array}{l}201 \\
\text { Esti- } \\
\text { mate }\end{array}$ & $\begin{array}{l}10 \\
95 \% \\
\text { UCB } \\
\end{array}$ & $\begin{array}{l}20 \\
\text { Esti- } \\
\text { mate }\end{array}$ & $\begin{array}{l}15 \\
95 \% \\
\text { UCB } \\
\end{array}$ & $\begin{array}{l}20 \\
\text { Esti- } \\
\text { mate }\end{array}$ & $\begin{array}{l}20 \\
95 \% \\
\text { UCB } \\
\end{array}$ & $\begin{array}{l}202 \\
\text { Esti- } \\
\text { mate }\end{array}$ & $\begin{array}{l}25 \\
95 \% \\
\text { UCB } \\
\end{array}$ \\
\hline \multirow[t]{3}{*}{ PORTS thin (P-scan data) } & 17,269 & Slope Fit & 250 & 673 & 1,010 & 784 & 1,160 & 1,112 & 1,583 & 1,506 & 2,064 & 1,954 & 2,596 & 2,442 & 3,175 \\
\hline & & & 62.5 & 0 & 0 & 0 & 0 & 0 & 0 & 0 & 0 & 0 & 0 & 0 & 0 \\
\hline & & & 0 & 0 & 0 & 0 & 0 & 0 & 0 & 0 & 0 & 0 & 0 & 0 & 0 \\
\hline \multirow[t]{3}{*}{ PORTS thin top } & 8,501 & Slope Fit & 250 & 7 & 45 & 8 & 48 & 10 & 55 & 13 & 63 & 16 & 71 & 19 & 81 \\
\hline & & & 62.5 & 0 & 0 & 0 & 0 & 0 & 0 & 0 & 0 & 0 & 0 & 0 & 0 \\
\hline & & & 0 & 0 & 0 & 0 & 0 & 0 & 0 & 0 & 0 & 0 & 0 & 0 & 0 \\
\hline \multirow[t]{3}{*}{ PORTS thin bottom } & 8,768 & Slope Fit & 250 & 7 & 43 & 8 & 47 & 10 & 57 & 14 & 67 & 18 & 79 & 22 & 92 \\
\hline & & & 62.5 & 0 & 0 & 0 & 0 & 0 & 0 & 0 & 0 & 0 & 0 & 0 & 0 \\
\hline & & & 0 & 0 & 0 & 0 & 0 & 0 & 0 & 0 & 0 & 0 & 0 & 0 & 0 \\
\hline \multirow[t]{3}{*}{ ETTP/PGDP/PORTS thick top } & 962 & Slope Fit & 500 & 1 & 3 & 1 & 3 & 1 & 3 & 1 & 3 & 1 & 3 & 1 & 4 \\
\hline & & & 62.5 & 0 & 0 & 0 & 0 & 0 & 0 & 0 & 0 & 0 & 0 & 0 & 0 \\
\hline & & & 0 & 0 & 0 & 0 & 0 & 0 & 0 & 0 & 0 & 0 & 0 & 0 & 0 \\
\hline \multirow[t]{3}{*}{ ETTP/PGDP/PORTS thick bottom } & 1,062 & Slope Fit & 500 & 6 & 14 & 6 & 14 & 6 & 14 & 6 & 15 & 6 & 16 & 6 & 16 \\
\hline & & & 62.5 & 0 & 0 & 0 & 0 & 0 & 0 & 0 & 0 & 0 & 0 & 0 & 0 \\
\hline & & & 0 & 0 & 0 & 0 & 0 & 0 & 0 & 0 & 0 & 0 & 0 & 0 & 0 \\
\hline \multirow{3}{*}{ ETTP/PGDP/PORTS thin skirted top } & 2,285 & Slope $=1$ & 250 & 20 & 53 & 24 & 60 & 34 & 81 & 47 & 104 & 62 & 130 & 81 & 159 \\
\hline & & & 62.5 & 0 & 1 & 0 & 1 & 0 & 2 & 0 & 3 & 1 & 4 & 1 & 5 \\
\hline & & & 0 & 0 & 0 & 0 & 0 & 0 & 1 & 0 & 1 & 0 & 2 & 0 & 2 \\
\hline \multirow{3}{*}{ ETTP/PGDP/PORTS thin skirted btm } & 2,487 & Slope $=1$ & 250 & 84 & 165 & 93 & 180 & 119 & 219 & 148 & 259 & 179 & 301 & 212 & 344 \\
\hline & & & 62.5 & 3 & 13 & 4 & 15 & 5 & 20 & 7 & 26 & 10 & 33 & 13 & 40 \\
\hline & & & 0 & 1 & 8 & 2 & 9 & 3 & 12 & 4 & 15 & 5 & 19 & 7 & 24 \\
\hline
\end{tabular}


Table 9A-cont'd. Summary of Indirect-Model Projections for Target Years and Minimum Thickness Criteria

\begin{tabular}{|c|c|c|c|c|c|c|c|c|c|c|c|c|c|c|c|c|c|}
\hline \multirow[b]{2}{*}{ Cylinder Population } & \multirow[b]{2}{*}{$\begin{array}{l}\text { Pop. } \\
\text { Total }\end{array}$} & \multirow[b]{2}{*}{ Model } & \multirow[b]{2}{*}{$\begin{array}{l}\text { Thick. } \\
\text { Spec } \\
\text { (mils) }\end{array}$} & \multicolumn{14}{|c|}{ Projected Number of Cylinders Below Thickness Criterion } \\
\hline & & & & $\begin{array}{r}20 \\
\text { Esti- } \\
\text { mate } \\
\end{array}$ & $\begin{array}{l}03 \\
95 \% \\
\text { UCB } \\
\end{array}$ & $\begin{array}{rr} & 20 \\
6 & \text { Esti- } \\
B & \text { mate } \\
\end{array}$ & $\begin{array}{l}05 \\
95 \% \\
\text { UCB } \\
\end{array}$ & $\begin{array}{r}2 \\
\text { Esti- } \\
\text { mat } \\
\end{array}$ & $\begin{array}{l}2010 \\
-95 \% \\
\text { e UCl } \\
\end{array}$ & & $\begin{array}{r}20 \\
\text { Esti- } \\
\text { mate } \\
\end{array}$ & $\begin{array}{l}015 \\
-95 \% \\
\text { UCB } \\
\end{array}$ & $\begin{array}{rr}2 \\
6 & \text { Esti } \\
\text { B mat } \\
\end{array}$ & & $\begin{array}{l}0 \\
5 \% \\
\text { UCB } \\
\end{array}$ & $\begin{array}{r}20 \\
\text { Esti- } \\
\text { mate } \\
\end{array}$ & $\begin{array}{l}025 \\
-95 \% \\
\text { UCB } \\
\end{array}$ \\
\hline \multirow{3}{*}{ ETTP/PGDP/PORTS thick skirted } & 2,024 & Slope $=1$ & 500 & 15 & 48 & 17 & 53 & 2. & 3 & 67 & 30 & 82 & 2 & 38 & 98 & 48 & $8 \quad 115$ \\
\hline & & & 62.5 & 0 & 3 & 0 & 3 & & 0 & 4 & 1 & 5 & 5 & 1 & 7 & 1 & 9 \\
\hline & & & 0 & 0 & 2 & 0 & 2 & & 0 & 3 & 0 & 4 & 4 & 1 & 5 & 1 & 7 \\
\hline \multirow[t]{3}{*}{ ETTP/PGDP/PORTS 1/2" (30" dia.) top } & 1,317 & Slope $=1$ & 100 & 3 & 16 & 4 & 18 & & 2 & 23 & 7 & 28 & 8 & 10 & 34 & 12 & 40 \\
\hline & & & 62.5 & 3 & 13 & 3 & 14 & & 4 & 18 & 6 & 23 & 3 & 7 & 28 & 9 & 33 \\
\hline & & & 0 & 2 & 9 & 2 & 10 & & 3 & 13 & 4 & 17 & 7 & 5 & 20 & 6 & 24 \\
\hline \multirow[t]{3}{*}{ ETTP/PGDP/PORTS 1/2" (30" dia.) btm } & 1,646 & Slope $=1$ & 100 & 7 & 29 & 8 & 33 & 10 & & 41 & 14 & 50 & 0 & 18 & 60 & 23 & 71 \\
\hline & & & 62.5 & 5 & 24 & 6 & 26 & & 8 & 33 & 10 & 41 & 1 & 14 & 50 & 17 & 59 \\
\hline & & & 0 & 3 & 17 & 3 & 19 & & 5 & 24 & 7 & 30 & 0 & 9 & 37 & 11 & 44 \\
\hline
\end{tabular}


Table 10A. Summary of Direct-Model Projections for Target Years and Thickness Criteria

\begin{tabular}{|c|c|c|c|c|c|c|c|c|}
\hline \multirow[b]{2}{*}{ Cylinder Population } & \multirow[b]{2}{*}{$\begin{array}{c}\text { Pop. } \\
\text { Total }\end{array}$} & & \multicolumn{6}{|c|}{ Projected Number of Cylinders Below Minimum Thickness Criteria } \\
\hline & & $\begin{array}{c}\text { Thick. } \\
\text { Spec } \\
\text { (mils) }\end{array}$ & $\begin{array}{l}2003 \\
\text { Esti- } \\
\text { mate }\end{array}$ & $\begin{array}{l}2005 \\
\text { Esti- } \\
\text { mate }\end{array}$ & $\begin{array}{l}2010 \\
\text { Esti- } \\
\text { mate }\end{array}$ & $\begin{array}{l}2015 \\
\text { Esti- } \\
\text { mate }\end{array}$ & $\begin{array}{l}2020 \\
\text { Esti- } \\
\text { mate }\end{array}$ & $\begin{array}{l}2025 \\
\text { Esti- } \\
\text { mate }\end{array}$ \\
\hline \multirow{3}{*}{ ETTP thin (P-scan data) } & 4,037 & 250 & 1,560 & 1,628 & 1,778 & 1,901 & 2,002 & 2,085 \\
\hline & & 62.5 & 15 & 16 & 19 & 21 & 23 & 25 \\
\hline & & 0 & 6 & 7 & 9 & 11 & 12 & 14 \\
\hline \multirow[t]{3}{*}{ ETTP thin, K-yard bottom } & 1,167 & 250 & 69 & 72 & 79 & 86 & 94 & 103 \\
\hline & & 62.5 & 3 & 3 & 4 & 4 & 4 & 5 \\
\hline & & 0 & 1 & 1 & 2 & 2 & 3 & 3 \\
\hline \multirow[t]{3}{*}{ ETTP thin, except $\mathrm{K}$-yard bottom } & 2,870 & 250 & 133 & 142 & 163 & 183 & 203 & 222 \\
\hline & & 62.5 & 5 & 5 & 7 & 8 & 9 & 10 \\
\hline & & 0 & 1 & 2 & 2 & 3 & 4 & 6 \\
\hline \multirow[t]{3}{*}{ PGDP thin (P-scan data) } & 32,231 & 250 & 1,944 & 2,164 & 2,754 & 3,306 & 3,851 & 4,375 \\
\hline & & 62.5 & 33 & 38 & 53 & 71 & 90 & 109 \\
\hline & & 0 & 13 & 16 & 21 & 28 & 37 & 48 \\
\hline \multirow{3}{*}{ PGDP thin bottom, former G-yard } & 1,983 & 250 & 81 & 86 & 96 & 106 & 116 & 124 \\
\hline & & 62.5 & 4 & 4 & 5 & 6 & 6 & 7 \\
\hline & & 0 & 1 & 1 & 2 & 2 & 3 & 4 \\
\hline \multirow[t]{3}{*}{ PGDP thin btm, excpt fmr G-yard } & 15,347 & 250 & 262 & 288 & 356 & 438 & 515 & 586 \\
\hline & & 62.5 & 9 & 10 & 13 & 18 & 24 & 32 \\
\hline & & 0 & 3 & 4 & 5 & 7 & 8 & 12 \\
\hline \multirow[t]{3}{*}{ PGDP thin top } & 14,901 & 250 & 219 & 240 & 295 & 354 & 420 & 481 \\
\hline & & 62.5 & 9 & 9 & 13 & 17 & 22 & 28 \\
\hline & & 0 & 3 & 3 & 4 & 6 & 8 & 10 \\
\hline
\end{tabular}


Table 10A-cont'd. Summary of Direct-Model Projections for Target Years and Thickness Criteria

\begin{tabular}{|c|c|c|c|c|c|c|c|c|}
\hline \multirow[b]{2}{*}{ Cylinder Population } & \multirow[b]{2}{*}{$\begin{array}{l}\text { Pop. } \\
\text { Total }\end{array}$} & \multirow[b]{2}{*}{$\begin{array}{c}\text { Thick. } \\
\text { Spec } \\
\text { (mils) }\end{array}$} & \multicolumn{6}{|c|}{ Projected Number of Cylinders Below Minimum Thickness Criteria } \\
\hline & & & $\begin{array}{l}2003 \\
\text { Esti- } \\
\text { mate }\end{array}$ & $\begin{array}{l}2005 \\
\text { Esti- } \\
\text { mate }\end{array}$ & $\begin{array}{l}2010 \\
\text { Esti- } \\
\text { mate }\end{array}$ & $\begin{array}{l}2015 \\
\text { Esti- } \\
\text { mate }\end{array}$ & $\begin{array}{l}2020 \\
\text { Esti- } \\
\text { mate }\end{array}$ & $\begin{array}{l}2025 \\
\text { Esti- } \\
\text { mate }\end{array}$ \\
\hline \multirow[t]{3}{*}{ PORTS thin (P-scan data) } & 17,269 & 250 & 1,095 & 1,222 & 1,537 & 1,833 & 2,136 & 2,400 \\
\hline & & 62.5 & 18 & 21 & 29 & 40 & 50 & 60 \\
\hline & & 0 & 7 & 8 & 11 & 15 & 20 & 26 \\
\hline \multirow[t]{3}{*}{ PORTS thin top } & 8,501 & 250 & 102 & 112 & 136 & 164 & 194 & 226 \\
\hline & & 62.5 & 4 & 5 & 7 & 9 & 12 & 15 \\
\hline & & 0 & 2 & 2 & 3 & 3 & 4 & 6 \\
\hline \multirow[t]{3}{*}{ PORTS thin bottom } & 8,768 & 250 & 104 & 115 & 141 & 170 & 202 & 236 \\
\hline & & 62.5 & 4 & 5 & 7 & 9 & 12 & 16 \\
\hline & & 0 & 2 & 2 & 3 & 3 & 4 & 6 \\
\hline \multirow[t]{3}{*}{ ETTP/PGDP/PORTS thick top } & 962 & 500 & 13 & 14 & 16 & 19 & 21 & 23 \\
\hline & & 62.5 & 0 & 0 & 0 & 0 & 0 & 0 \\
\hline & & 0 & 0 & 0 & 0 & 0 & 0 & 0 \\
\hline \multirow[t]{3}{*}{ ETTP/PGDP/PORTS thick bottom } & 1,062 & 500 & 14 & 15 & 18 & 20 & 23 & 25 \\
\hline & & 62.5 & 0 & 0 & 0 & 0 & 0 & 0 \\
\hline & & 0 & 0 & 0 & 0 & 0 & 0 & 0 \\
\hline \multirow[t]{3}{*}{ ETTP/PGDP/PORTS thin skirted top } & 2,285 & 250 & 47 & 50 & 57 & 64 & 71 & 78 \\
\hline & & 62.5 & 3 & 4 & 5 & 5 & 6 & 6 \\
\hline & & 0 & 1 & 1 & 1 & 2 & 3 & 4 \\
\hline \multirow[t]{3}{*}{ ETTP/PGDP/PORTS thin skirted btm } & 2,487 & 250 & 62 & 66 & 75 & 85 & 93 & 102 \\
\hline & & 62.5 & 4 & 5 & 6 & 6 & 7 & 7 \\
\hline & & 0 & 1 & 1 & 2 & 3 & 4 & 4 \\
\hline
\end{tabular}


Table 10A-cont'd. Summary of Direct-Model Projections for Target Years and Thickness Criteria

\begin{tabular}{|c|c|c|c|c|c|c|c|c|}
\hline \multirow[b]{2}{*}{ Cylinder Population } & \multirow[b]{2}{*}{$\begin{array}{l}\text { Pop. } \\
\text { Total }\end{array}$} & & \multicolumn{6}{|c|}{ Projected Number of Cylinders Below Minimum Thickness Criteria } \\
\hline & & $\begin{array}{c}\text { Thick. } \\
\text { Spec } \\
\text { (mils) }\end{array}$ & $\begin{array}{l}2003 \\
\text { Esti- } \\
\text { mate }\end{array}$ & $\begin{array}{l}2005 \\
\text { Esti- } \\
\text { mate }\end{array}$ & $\begin{array}{l}2010 \\
\text { Esti- } \\
\text { mate }\end{array}$ & $\begin{array}{l}2015 \\
\text { Esti- } \\
\text { mate }\end{array}$ & $\begin{array}{l}2020 \\
\text { Esti- } \\
\text { mate }\end{array}$ & $\begin{array}{l}2025 \\
\text { Esti- } \\
\text { mate }\end{array}$ \\
\hline \multirow[t]{3}{*}{ ETTP/PGDP/PORTS thick skirted } & 2,024 & 500 & 6 & 6 & 7 & 8 & 10 & 11 \\
\hline & & 62.5 & 0 & 0 & 0 & 0 & 0 & 0 \\
\hline & & 0 & 0 & 0 & 0 & 0 & 0 & 0 \\
\hline \multirow[t]{3}{*}{ ETTP/PGDP/PORTS 1/2" (30" dia.) top } & 1,317 & 100 & 1 & 1 & 1 & 1 & 1 & 2 \\
\hline & & 62.5 & 0 & 0 & 1 & 1 & 1 & 1 \\
\hline & & 0 & 0 & 0 & 0 & 0 & 0 & 0 \\
\hline \multirow[t]{3}{*}{ ETTP/PGDP/PORTS 1/2" (30" dia.) btm } & 1,646 & 100 & 1 & 1 & 1 & 1 & 2 & 2 \\
\hline & & 62.5 & 0 & 0 & 1 & 1 & 1 & 1 \\
\hline & & 0 & 0 & 0 & 0 & 0 & 0 & 1 \\
\hline
\end{tabular}


In the indirect modeling approach, for the various cylinder subpopulations, the statistical distributions of $M(t)$ are assumed to be lognormal, and regressions are used to estimate the parameters of the respective lognormal distributions. These estimates and therefore the corresponding projections do not incorporate adjustments to account for statistical variability in model parameter estimates. The projections may be conservative for other reasons but not because of statistical adjustments to account for error in the parameter estimates. However, in addition to the projections, Table 9A also contains upper confidence limits (UCLs) for the projections. The numbers in the columns labeled "Estimate" are point estimates computed from the regression estimates; the numbers labeled "95\% UCL" are approximate upper 95\% confidence limits computed using the method described in Appendix B. The confidence limits take into account variability in the regression parameter estimates, though the mathematics underlying them is based on conservative approximations.

The direct-model projections in Table 10A are based on the same large-sample approximations that are used to derive the LCLs plotted in Figures 18-34 for individual predicted values. Those confidence limits are nonparametric analogs of the usual normal-theory LCLs for individual predicted values, which are also plotted in the Figures 18-34. Both the normal and nonparametric confidence limits are computed by determining, for a specified probability $p$ (e.g., .99) and age $t$, a corresponding limit $L$ such that $\operatorname{Prob}(M(t)>$ $L) \approx p$.

Like their normal-theory analogs, the nonparametric limits account for statistical error in the regression parameter estimates. However, for the nonparametric limits, the underlying regression error distribution is not assumed to be normal, but rather is estimated, from the empirical distribution of the regression residuals, in a way that is asymptotically exact. Thus, except when the distribution of regression errors is exactly normal, both the normal and large-sample confidence limits are approximate.

For the direct-model projections, instead of specifying a probability $p$ (e.g., .99) and an age $t$, and determining a corresponding limit $L$ such that $\operatorname{Prob}(M(t)>L) \approx p$, a limit $z$ is specified along with an age $t$, and the same approach is then used to determine a $p$ such that $\operatorname{Prob}(M(t)>z) \approx p$. This $p$ is an estimate of $\operatorname{Prob}(M(t)>z)$ and can thus be used in projection estimates computed as in Equation (2.1).

The direct model projections incorporate adjustments that account for statistical error in the regression parameter estimates. In that sense they are more like the confidence bounds for the indirect-model projections, than the indirect-model projections themselves. However, the approximations in the directmodel approach are not as conservative as the approximations used to derive the indirect-model confidence bounds. Thus the direct-model projections are likely to be more conservative than the indirect-model projections, but not as conservative as the confidence limits for the indirect-model projections.

Tables 9A and 10A show clearly that projections of the numbers of noncompliant cylinders are much higher for the P-scan groups than for the manual UT groups. Thus, in the following discussion, the P-scan results are deprecated in favor of the more recent manual UT scan data. Tables 9A and 10A both project that many cylinders will fail the upper (e.g., 250 or 500 mil) thickness specifications. The validation analysis in Section 6 shows that these projections are reasonable. The validation analysis is less conclusive about results for thickness specifications less than 250 mils, however, because so few cylinders are ever observed to fail these criteria. Tables 9A and 10A do project numerous breaches in 2003 and later years, however, and although breaches have occurred in the past, and despite random variations, the numbers of breaches predicted in the tables seem too high—it is unlikely that breaches of this frequency would go unnoticed, even if they were not detected in the UT scanning itself. 
Reasons for high projections include:

- Many of the cylinders were not sampled randomly (e.g., using a random number generator), but were selected "quasi-randomly" or, as is natural in inspections, with purposive focus on groups thought to be at higher risk.

- The cylinder groupings (subpopulations) only roughly approximate the complete storage location history of cylinders. Because cylinders are typically moved from time to time, the "locations" associated with the cylinder groupings would be better represented as combinations of locations.

- When cylinders are moved, they are usually moved to improved storage locations.

The complete storage histories are not always available, however, in any form (let alone electronic), and the accounting in such an approach would be much more difficult than the direct or indirect approaches used for this report.

Disregarding P-scan results and summing projected numbers of breaches in Table 9A shows that the indirect-model analysis predicts a total of ten breaches in FY03. This figure is a point estimate-no adjustment is made for statistical error in the regression parameter estimates. The corresponding projection confidence limits in the table account for error in the parameter estimates. However, the sum of those confidence limits is 40 , which seems too conservative to be useful. It would be extremely unlikely that 40 breaches would go unnoticed, even without any UT scanning at all. Therefore, only the indirect-model point estimate projections and not the confidence limits will be used to compute projections of numbers of cylinders that fail various thickness criteria, which are considered in Section 5.

The sum of the direct-model projections (Table 10A) is 15. To some extent, the direct model projections account for statistical error in the parameter estimates and should therefore be somewhat higher (more conservative) than the indirect model point estimate projections. Nevertheless, like the indirect model projections, the direct model projections also seem too high.

Because of updating of the cylinder subpopulation definitions, deprecation of the P-scan data, and the addition of new data for measurements made in FY02, the projected numbers of breaches are considerably lower than the corresponding projections for FY02 computed for the FY02 report (Schmoyer and Lyon, 2002). From the FY02 report, excluding breaches predicted from P-scan data results, 67 breaches were projected (for FY02) for the indirect model, and 23 were predicted for the direct model. But despite the improvement, the FY03 projections still appear to be conservative.

Projections with Outlier Exclusions. Tables 9B and 10B were computed to show the very substantial influence that only a few data points have in both the direct and indirect model analyses. Table 9B is the analog of Table 9A for the indirect model, computed after excluding observations for which the minimum pit depth exceeds 160 mils. Table 10B the analog of Table 10A for the direct model, computed after excluding observations for which the regression residual is less than -20 mils. Note that the 160 mil and -20 mil thresholds are subjective; different cutoffs could also be considered. The excluded cylinders are listed in Table 11. The number of outliers dropped is $27 \mathrm{for}$ the indirect model and 24 for the direct model ( 23 of which are common to both models). When the outliers are dropped, the number of predicted breaches drops from 10 to 3 for the indirect model and from 15 to 6 for the direct model. 
Table 9B. Summary of Indirect-Model Projections for Data with Outlier Exclusions

\begin{tabular}{|c|c|c|c|c|c|c|c|c|c|c|c|c|c|c|c|}
\hline \multirow[b]{2}{*}{ Population } & \multirow[b]{2}{*}{$\begin{array}{c}\text { Pop. } \\
\text { Total } \\
\end{array}$} & \multirow[b]{2}{*}{ Model } & \multirow[b]{2}{*}{$\begin{array}{c}\text { Thick. } \\
\text { Spec } \\
\text { (mils) }\end{array}$} & \multicolumn{12}{|c|}{ Projected Number of Cylinders Below Thickness Criterion } \\
\hline & & & & $\begin{array}{l}200 \\
\text { Esti- } \\
\text { mate }\end{array}$ & $\begin{array}{l}03 \\
95 \% \\
\text { UCB } \\
\end{array}$ & $\begin{array}{r}20 \\
\text { Esti- } \\
\text { mate } \\
\end{array}$ & $\begin{array}{l}05 \\
95 \% \\
\text { UCB } \\
\end{array}$ & $\begin{array}{l}20 \\
\text { Esti- } \\
\text { mate }\end{array}$ & $\begin{array}{l}10 \\
95 \% \\
\text { UCB } \\
\end{array}$ & $\begin{array}{r}20 \\
\text { Esti- } \\
\text { mate }\end{array}$ & $\begin{array}{c}015 \\
95 \% \\
\text { UCB } \\
\end{array}$ & $\begin{array}{l}20 \\
\text { Esti- } \\
\text { mate }\end{array}$ & $\begin{array}{l}020 \\
95 \% \\
\text { UCB } \\
\end{array}$ & $\begin{array}{l}20 \\
\text { Esti- } \\
\text { mate }\end{array}$ & $\begin{array}{l}25 \\
95 \% \\
\text { UCB }\end{array}$ \\
\hline \multirow[t]{3}{*}{ ETTP thin (P-scan data) } & 4,037 & Slope $=1$ & 250 & 1,553 & 1,814 & 1,738 & 1,987 & 2,188 & 2,483 & 2,595 & 2,910 & 2,938 & 3,248 & 3,215 & 3,499 \\
\hline & & & 62.5 & 1 & 8 & 1 & 11 & 4 & 22 & 8 & 39 & 16 & 64 & 28 & 99 \\
\hline & & & 0 & 0 & 2 & 0 & 2 & 0 & 5 & 1 & 10 & 3 & 18 & 5 & 29 \\
\hline \multirow[t]{3}{*}{ ETTP thin, K-yard bottom } & 1,167 & Slope $=1$ & 250 & 77 & 131 & 86 & 143 & 110 & 172 & 135 & 202 & 161 & 232 & 188 & 262 \\
\hline & & & 62.5 & 1 & 6 & 2 & 7 & 3 & 10 & 4 & 13 & 5 & 17 & 7 & 21 \\
\hline & & & 0 & 1 & 3 & 1 & 4 & 1 & 5 & 2 & 7 & 2 & 9 & 3 & 12 \\
\hline \multirow[t]{3}{*}{ ETTP thin, except K-yard bottom } & 2,870 & Slope $=1$ & 250 & 113 & 235 & 129 & 260 & 171 & 322 & 218 & 387 & 268 & 452 & 320 & 517 \\
\hline & & & 62.5 & 3 & 16 & 4 & 18 & 5 & 25 & 8 & 33 & 11 & 43 & 15 & 54 \\
\hline & & & 0 & 1 & 9 & 2 & 10 & 3 & 14 & 4 & 19 & 5 & 25 & 7 & 32 \\
\hline \multirow{3}{*}{ PGDP thin (P-scan data) } & 32,231 & Slope Fit & 250 & 2,509 & 4,181 & 2,928 & 4,789 & 4,186 & 6,481 & 5,745 & 8,345 & 7,555 & 10,314 & 9,526 & 12,386 \\
\hline & & & 62.5 & 1 & 8 & 1 & 10 & 3 & 20 & 6 & 36 & 10 & 60 & 18 & 96 \\
\hline & & & 0 & 0 & 1 & 0 & 2 & 0 & 4 & 1 & 7 & 2 & 13 & 3 & 22 \\
\hline \multirow[t]{3}{*}{ PGDP thin bottom, former G-yard } & 1,983 & Slope Fit & 250 & 32 & 119 & 34 & 127 & 40 & 149 & 47 & 173 & 53 & 198 & 59 & 226 \\
\hline & & & 62.5 & 0 & 3 & 0 & 3 & 0 & 4 & 0 & 5 & 0 & 7 & 0 & 9 \\
\hline & & & 0 & 0 & 1 & 0 & 1 & 0 & 2 & 0 & 3 & 0 & 3 & 0 & 4 \\
\hline \multirow[t]{3}{*}{ PGDP thin btm, excpt fmr G-yard } & 15,347 & Slope Fit & 250 & 67 & 249 & 76 & 268 & 101 & 317 & 129 & 367 & 159 & 421 & 190 & 481 \\
\hline & & & 62.5 & 0 & 3 & 0 & 3 & 0 & 4 & 1 & 5 & 1 & 6 & 1 & 7 \\
\hline & & & 0 & 0 & 1 & 0 & 1 & 0 & 1 & 0 & 2 & 0 & 2 & 0 & 3 \\
\hline \multirow[t]{3}{*}{ PGDP thin top } & 14,901 & Slope Fit & 250 & 14 & 100 & 15 & 102 & 19 & 109 & 23 & 117 & 26 & 126 & 30 & 139 \\
\hline & & & 62.5 & 0 & 0 & 0 & 0 & 0 & 0 & 0 & 0 & 0 & 0 & 0 & 1 \\
\hline & & & 0 & 0 & 0 & 0 & 0 & 0 & 0 & 0 & 0 & 0 & 0 & 0 & 0 \\
\hline
\end{tabular}


Table 9B-cont'd. Summary of Indirect-Model Projections for Data with Outlier Exclusions

\begin{tabular}{|c|c|c|c|c|c|c|c|c|c|c|c|c|c|c|c|}
\hline \multirow[b]{2}{*}{ Population } & \multirow[b]{2}{*}{$\begin{array}{l}\text { Pop. } \\
\text { Total }\end{array}$} & \multirow[b]{2}{*}{ Model } & \multirow[b]{2}{*}{$\begin{array}{c}\text { Thick. } \\
\text { Spec } \\
\text { (mils) }\end{array}$} & \multicolumn{12}{|c|}{ Projected Number of Cylinders Below Thickness Criterion } \\
\hline & & & & $\begin{array}{r}20 \\
\text { Esti- } \\
\text { mate }\end{array}$ & $\begin{array}{l}03 \\
95 \% \\
\text { UCB } \\
\end{array}$ & $\begin{array}{r}20 \\
\text { Esti- } \\
\text { mate }\end{array}$ & $\begin{array}{l}05 \\
95 \% \\
\text { UCB } \\
\end{array}$ & $\begin{array}{l}20 \\
\text { Esti- } \\
\text { mate }\end{array}$ & $\begin{array}{l}10 \\
95 \% \\
\text { UCB } \\
\end{array}$ & $\begin{array}{r}20 \\
\text { Esti- } \\
\text { mate }\end{array}$ & $\begin{array}{l}15 \\
95 \% \\
\text { UCB } \\
\end{array}$ & $\begin{array}{l}20 \\
\text { Esti- } \\
\text { mate } \\
\end{array}$ & $\begin{array}{l}020 \\
95 \% \\
\text { UCB } \\
\end{array}$ & $\begin{array}{r}202 \\
\text { Esti- } \\
\text { mate }\end{array}$ & $\begin{array}{l}25 \\
95 \% \\
\text { UCB }\end{array}$ \\
\hline \multirow[t]{3}{*}{ PORTS thin (P-scan data) } & 17,269 & Slope Fit & 250 & 673 & 1,010 & 784 & 1,160 & 1,112 & 1,583 & 1,506 & 2,064 & 1,954 & 2,596 & 52,442 & 3,175 \\
\hline & & & 62.5 & 0 & 0 & 0 & 0 & 0 & 0 & 0 & 0 & 0 & 0 & 0 & 0 \\
\hline & & & 0 & 0 & 0 & 0 & 0 & 0 & 0 & 0 & 0 & 0 & 0 & 0 & 0 \\
\hline \multirow[t]{3}{*}{ PORTS thin top } & 8,501 & Slope Fit & 250 & 7 & 45 & 8 & 48 & 10 & 55 & 13 & 63 & 16 & 71 & 19 & 81 \\
\hline & & & 62.5 & 0 & 0 & 0 & 0 & 0 & 0 & 0 & 0 & 0 & 0 & 0 & 0 \\
\hline & & & 0 & 0 & 0 & 0 & 0 & 0 & 0 & 0 & 0 & 0 & 0 & 0 & 0 \\
\hline \multirow[t]{3}{*}{ PORTS thin bottom } & 8,768 & Slope Fit & 250 & 7 & 43 & 8 & 47 & 10 & 57 & 14 & 67 & 18 & 79 & 22 & 92 \\
\hline & & & 62.5 & 0 & 0 & 0 & 0 & 0 & 0 & 0 & 0 & 0 & 0 & 0 & 0 \\
\hline & & & 0 & 0 & 0 & 0 & 0 & 0 & 0 & 0 & 0 & 0 & 0 & 0 & 0 \\
\hline \multirow[t]{3}{*}{ ETTP/PGDP/PORTS thick top } & 962 & Slope Fit & 500 & 1 & 3 & 1 & 3 & 1 & 3 & 1 & 3 & 1 & 3 & 1 & 4 \\
\hline & & & 62.5 & 0 & 0 & 0 & 0 & 0 & 0 & 0 & 0 & 0 & 0 & 0 & 0 \\
\hline & & & 0 & 0 & 0 & 0 & 0 & 0 & 0 & 0 & 0 & 0 & 0 & 0 & 0 \\
\hline \multirow[t]{3}{*}{ ETTP/PGDP/PORTS thick bottom } & 1,062 & Slope Fit & 500 & 6 & 14 & 6 & 14 & 6 & 14 & 6 & 15 & 6 & 16 & 6 & 16 \\
\hline & & & 62.5 & 0 & 0 & 0 & 0 & 0 & 0 & 0 & 0 & 0 & 0 & 0 & 0 \\
\hline & & & 0 & 0 & 0 & 0 & 0 & 0 & 0 & 0 & 0 & 0 & 0 & 0 & 0 \\
\hline \multirow[t]{3}{*}{ ETTP/PGDP/PORTS thin skirted top } & 2,285 & Slope $=1$ & 250 & 20 & 53 & 24 & 60 & 34 & 81 & 47 & 104 & 62 & 130 & 81 & 159 \\
\hline & & & 62.5 & 0 & 1 & 0 & 1 & 0 & 2 & 0 & 3 & 1 & 4 & 1 & 5 \\
\hline & & & 0 & 0 & 0 & 0 & 0 & 0 & 1 & 0 & 1 & 0 & 2 & 0 & 2 \\
\hline \multirow[t]{3}{*}{ ETTP/PGDP/PORTS thin skirted btm } & 2,487 & Slope $=1$ & 250 & 84 & 165 & 93 & 180 & 119 & 219 & 148 & 259 & 179 & 301 & 212 & 344 \\
\hline & & & 62.5 & 3 & 13 & 4 & 15 & 5 & 20 & 7 & 26 & 10 & 33 & 13 & 40 \\
\hline & & & 0 & 1 & 8 & 2 & 9 & 3 & 12 & 4 & 15 & 5 & 19 & 7 & 24 \\
\hline
\end{tabular}


Table 9B-cont'd. Summary of Indirect-Model Projections for Data with Outlier Exclusions

\begin{tabular}{|c|c|c|c|c|c|c|c|c|c|c|c|c|c|c|c|c|c|c|}
\hline \multirow[b]{2}{*}{ Population } & \multirow[b]{2}{*}{$\begin{array}{l}\text { Pop. } \\
\text { Total }\end{array}$} & \multirow[b]{2}{*}{ Model } & \multicolumn{16}{|c|}{ Projected Number of Cylinders Below Thickness Criterion } \\
\hline & & & $\begin{array}{l}\text { Thick. } \\
\text { Spec } \\
\text { (mils) }\end{array}$ & $\begin{array}{r}20 \\
\text { Esti- } \\
\text { mate } \\
\end{array}$ & $\begin{array}{l}03 \\
95 \% \\
\text { UCB } \\
\end{array}$ & $\begin{array}{r}20 \\
\text { Esti- } \\
\text { mate }\end{array}$ & $\begin{array}{l}005 \\
-95 \% \\
\text { UCI } \\
\end{array}$ & & $\begin{array}{r}20 \\
\text { Esti- } \\
\text { mate } \\
\end{array}$ & $\begin{array}{l}10 \\
95 \% \\
\text { UCB } \\
\end{array}$ & $\begin{array}{r}2 \\
\text { Esti- } \\
\text { matt } \\
\end{array}$ & $\begin{array}{r}015 \\
-950 \\
\text { e UC } \\
\end{array}$ & $\begin{array}{l}\% \\
\text { CB } \\
\end{array}$ & $\begin{array}{r}20 \\
\text { Esti- } \\
\text { mate } \\
\end{array}$ & $\begin{array}{l}920 \\
95 \% \\
\text { UCB } \\
\end{array}$ & $\begin{array}{ll} & 20 \\
\text { \% } & \text { Esti- } \\
\text { B } & \text { mate } \\
\end{array}$ & $\begin{array}{l}2025 \\
-9 \\
\text { te } U \\
\end{array}$ & $\begin{array}{l}5 \% \\
5 \% \\
\end{array}$ \\
\hline \multirow{3}{*}{ ETTP/PGDP/PORTS thick skirted } & 2,024 & Slope $=1$ & 500 & 15 & 48 & 17 & 7 & 3 & 23 & 67 & 30 & 0 & 82 & 38 & & 8 & 48 & 115 \\
\hline & & & 62.5 & 0 & 3 & 0 & 0 & 3 & 0 & 4 & 4 & 1 & 5 & 1 & 7 & 7 & 1 & 9 \\
\hline & & & 0 & 0 & 2 & 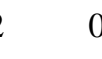 & 0 & 2 & 0 & 3 & 3 & 0 & 4 & 1 & 5 & 5 & 1 & 7 \\
\hline \multirow[t]{3}{*}{ ETTP/PGDP/PORTS 1/2" (30" dia.) top } & 1,317 & Slope $=1$ & 100 & 0 & 1 & 0 & 0 & 2 & 0 & 2 & 2 & 0 & 3 & 1 & 5 & 5 & 1 & 6 \\
\hline & & & 62.5 & 0 & 1 & . & 0 & 1 & 0 & 2 & 2 & 0 & 2 & 0 & 3 & 3 & 1 & 4 \\
\hline & & & 0 & 0 & 1 & . & 0 & 1 & 0 & 1 & 1 & 0 & 1 & 0 & 2 & 2 & 0 & 3 \\
\hline \multirow[t]{3}{*}{ ETTP/PGDP/PORTS 1/2" (30" dia.) btm } & 1,646 & Slope $=1$ & 100 & 1 & 9 & 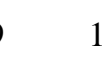 & 1 & 0 & 2 & 14 & 4 & 3 & 18 & 4 & 23 & 3 & 6 & 28 \\
\hline & & & 62.5 & 1 & 6 & 5 & 1 & 7 & 1 & 10 & 0 & 2 & 14 & 3 & 18 & 8 & 4 & 22 \\
\hline & & & 0 & 0 & 4 & 0 & 0 & 5 & 1 & & 7 & 1 & 9 & 2 & 12 & 2 & 2 & 15 \\
\hline
\end{tabular}


Table 10B. Summary of Direct-Model Projections for Data with Outlier Exclusions

\begin{tabular}{|c|c|c|c|c|c|c|c|c|}
\hline \multirow[b]{2}{*}{ Cylinder Population } & \multirow[b]{2}{*}{$\begin{array}{l}\text { Pop. } \\
\text { Total }\end{array}$} & \multirow[b]{2}{*}{$\begin{array}{l}\text { Thick. } \\
\text { Spec } \\
\text { (mils) }\end{array}$} & \multicolumn{6}{|c|}{ Projected Number of Cylinders Below Minimum Thickness Criteria } \\
\hline & & & $\begin{array}{l}2003 \\
\text { Esti- } \\
\text { mate }\end{array}$ & $\begin{array}{l}2005 \\
\text { Esti- } \\
\text { mate }\end{array}$ & $\begin{array}{l}2010 \\
\text { Esti- } \\
\text { mate }\end{array}$ & $\begin{array}{l}2015 \\
\text { Esti- } \\
\text { mate }\end{array}$ & $\begin{array}{l}2020 \\
\text { Esti- } \\
\text { mate }\end{array}$ & $\begin{array}{l}2025 \\
\text { Esti- } \\
\text { mate }\end{array}$ \\
\hline \multirow[t]{3}{*}{ ETTP thin (P-scan data) } & 4,037 & 250 & 1,211 & 1,269 & 1,404 & 1,521 & 1,621 & 1,706 \\
\hline & & 62.5 & 1 & 1 & 1 & 1 & 1 & 1 \\
\hline & & 0 & 1 & 1 & 1 & 1 & 1 & 1 \\
\hline \multirow[t]{3}{*}{ ETTP thin, K-yard bottom } & 1,167 & 250 & 61 & 64 & 71 & 78 & 86 & 95 \\
\hline & & 62.5 & 0 & 0 & 0 & 0 & 0 & 0 \\
\hline & & 0 & 0 & 0 & 0 & 0 & 0 & 0 \\
\hline \multirow[t]{3}{*}{ ETTP thin, except K-yard bottom } & 2,870 & 250 & 102 & 110 & 129 & 148 & 165 & 182 \\
\hline & & 62.5 & 0 & 0 & 0 & 0 & 0 & 0 \\
\hline & & 0 & 0 & 0 & 0 & 0 & 0 & 0 \\
\hline \multirow{3}{*}{ PGDP thin (P-scan data) } & 32,231 & 250 & 1,723 & 1,944 & 2,535 & 3,086 & 3,632 & 4,160 \\
\hline & & 62.5 & 5 & 5 & 5 & 5 & 5 & 5 \\
\hline & & 0 & 5 & 5 & 5 & 5 & 5 & 5 \\
\hline \multirow{3}{*}{ PGDP thin bottom, former G-yard } & 1,983 & 250 & 67 & 72 & 82 & 92 & 102 & 110 \\
\hline & & 62.5 & 0 & 0 & 0 & 0 & 0 & 0 \\
\hline & & 0 & 0 & 0 & 0 & 0 & 0 & 0 \\
\hline \multirow[t]{3}{*}{ PGDP thin btm, excpt fmr G-yard } & 15,347 & 250 & 142 & 163 & 226 & 301 & 376 & 442 \\
\hline & & 62.5 & 2 & 2 & 2 & 2 & 2 & 2 \\
\hline & & 0 & 2 & 2 & 2 & 2 & 2 & 2 \\
\hline \multirow[t]{3}{*}{ PGDP thin top } & 14,901 & 250 & 117 & 136 & 188 & 247 & 314 & 375 \\
\hline & & 62.5 & 2 & 2 & 2 & 2 & 2 & 2 \\
\hline & & 0 & 2 & 2 & 2 & 2 & 2 & 2 \\
\hline
\end{tabular}


Table 10B-cont'd. Summary of Direct-Model Projections for Data with Outlier Exclusions

\begin{tabular}{|c|c|c|c|c|c|c|c|c|}
\hline \multirow[b]{2}{*}{ Cylinder Population } & \multirow[b]{2}{*}{$\begin{array}{c}\text { Pop. } \\
\text { Total }\end{array}$} & \multirow[b]{2}{*}{$\begin{array}{l}\text { Thick. } \\
\text { Spec } \\
\text { (mils) }\end{array}$} & \multicolumn{6}{|c|}{ Projected Number of Cylinders Below Minimum Thickness Criteria } \\
\hline & & & $\begin{array}{l}2003 \\
\text { Esti- } \\
\text { mate }\end{array}$ & $\begin{array}{l}2005 \\
\text { Esti- } \\
\text { mate }\end{array}$ & $\begin{array}{l}2010 \\
\text { Esti- } \\
\text { mate }\end{array}$ & $\begin{array}{l}2015 \\
\text { Esti- } \\
\text { mate }\end{array}$ & $\begin{array}{l}2020 \\
\text { Esti- } \\
\text { mate }\end{array}$ & $\begin{array}{l}2025 \\
\text { Esti- } \\
\text { mate }\end{array}$ \\
\hline \multirow[t]{3}{*}{ PORTS thin (P-scan data) } & 17,269 & 250 & 977 & 1,104 & 1,420 & 1,715 & 2,019 & 2,284 \\
\hline & & 62.5 & 3 & 3 & 3 & 3 & 3 & 3 \\
\hline & & 0 & 3 & 3 & 3 & 3 & 3 & 3 \\
\hline \multirow[t]{3}{*}{ PORTS thin top } & 8,501 & 250 & 45 & 52 & 76 & 103 & 133 & 166 \\
\hline & & 62.5 & 1 & 1 & 1 & 1 & 1 & 1 \\
\hline & & 0 & 1 & 1 & 1 & 1 & 1 & 1 \\
\hline \multirow[t]{3}{*}{ PORTS thin bottom } & 8,768 & 250 & 43 & 51 & 75 & 104 & 134 & 167 \\
\hline & & 62.5 & 1 & 1 & 1 & 1 & 1 & 1 \\
\hline & & 0 & 1 & 1 & 1 & 1 & 1 & 1 \\
\hline \multirow{3}{*}{ ETTP/PGDP/PORTS thick top } & 962 & 500 & 6 & 7 & 9 & 12 & 14 & 16 \\
\hline & & 62.5 & 0 & 0 & 0 & 0 & 0 & 0 \\
\hline & & 0 & 0 & 0 & 0 & 0 & 0 & 0 \\
\hline \multirow[t]{3}{*}{ ETTP/PGDP/PORTS thick bottom } & 1,062 & 500 & 6 & 7 & 9 & 12 & 14 & 16 \\
\hline & & 62.5 & 0 & 0 & 0 & 0 & 0 & 0 \\
\hline & & 0 & 0 & 0 & 0 & 0 & 0 & 0 \\
\hline \multirow[t]{3}{*}{ ETTP/PGDP/PORTS thin skirted top } & 2,285 & 250 & 31 & 33 & 40 & 48 & 55 & 61 \\
\hline & & 62.5 & 0 & 0 & 0 & 0 & 0 & 0 \\
\hline & & 0 & 0 & 0 & 0 & 0 & 0 & 0 \\
\hline \multirow{3}{*}{ ETTP/PGDP/PORTS thin skirted btm } & 2,487 & 250 & 44 & 48 & 58 & 67 & 76 & 85 \\
\hline & & 62.5 & 0 & 0 & 0 & 0 & 0 & 0 \\
\hline & & 0 & 0 & 0 & 0 & 0 & 0 & 0 \\
\hline
\end{tabular}


Table 10B-cont'd. Summary of Direct-Model Projections for Data with Outlier Exclusions

\begin{tabular}{|c|c|c|c|c|c|c|c|c|}
\hline \multirow[b]{2}{*}{ Cylinder Population } & \multirow[b]{2}{*}{$\begin{array}{c}\text { Pop. } \\
\text { Total } \\
\end{array}$} & \multirow[b]{2}{*}{$\begin{array}{l}\text { Thick. } \\
\text { Spec } \\
\text { (mils) }\end{array}$} & \multicolumn{6}{|c|}{ Projected Number of Cylinders Below Minimum Thickness Criteria } \\
\hline & & & $\begin{array}{l}2003 \\
\text { Esti- } \\
\text { mate }\end{array}$ & $\begin{array}{l}2005 \\
\text { Esti- } \\
\text { mate }\end{array}$ & $\begin{array}{l}2010 \\
\text { Esti- } \\
\text { mate }\end{array}$ & $\begin{array}{l}2015 \\
\text { Esti- } \\
\text { mate }\end{array}$ & $\begin{array}{l}2020 \\
\text { Esti- } \\
\text { mate }\end{array}$ & $\begin{array}{l}2025 \\
\text { Esti- } \\
\text { mate }\end{array}$ \\
\hline \multirow[t]{3}{*}{ ETTP/PGDP/PORTS thick skirted } & 2,024 & 500 & 0 & 0 & 0 & 0 & 0 & 0 \\
\hline & & 62.5 & 0 & 0 & 0 & 0 & 0 & 0 \\
\hline & & 0 & 0 & 0 & 0 & 0 & 0 & 0 \\
\hline \multirow[t]{3}{*}{ ETTP/PGDP/PORTS 1/2" (30" dia.) top } & 1,317 & 100 & 0 & 0 & 0 & 0 & 0 & 0 \\
\hline & & 62.5 & 0 & 0 & 0 & 0 & 0 & 0 \\
\hline & & 0 & 0 & 0 & 0 & 0 & 0 & 0 \\
\hline \multirow[t]{3}{*}{ ETTP/PGDP/PORTS 1/2" (30" dia.) btm } & 1,646 & 100 & 0 & 0 & 0 & 0 & 0 & 0 \\
\hline & & 62.5 & 0 & 0 & 0 & 0 & 0 & 0 \\
\hline & & 0 & 0 & 0 & 0 & 0 & 0 & 0 \\
\hline
\end{tabular}


Table 9C. Indirect-Model Projections (Projected Proportions) for Target Years and Minimum Thickness Criteria

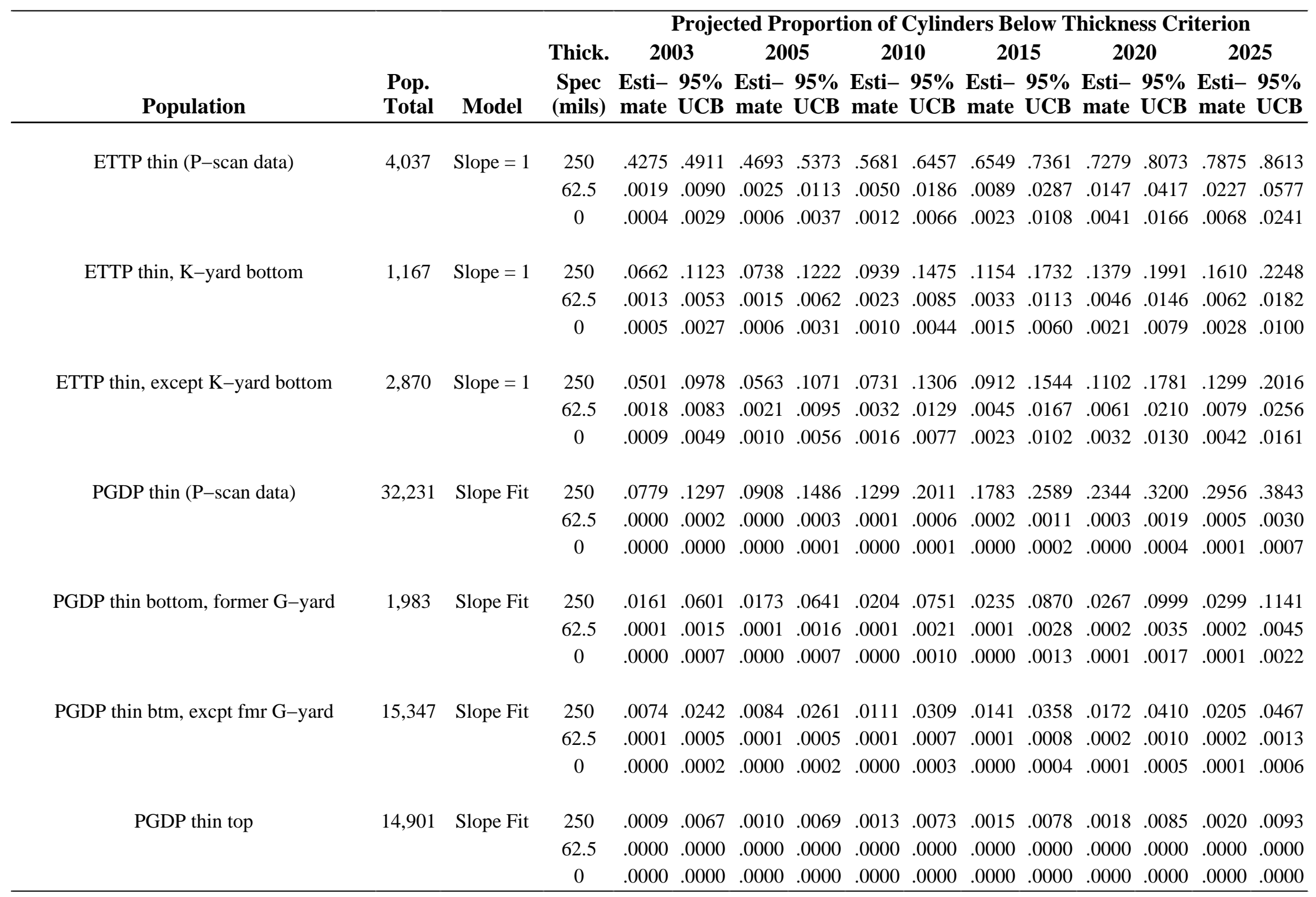


Table 9C-cont'd. Indirect-Model Projections (Projected Proportions) for Target Years and Minimum Thickness Criteria

\begin{tabular}{|c|c|c|c|c|c|c|c|c|c|c|c|c|c|c|c|}
\hline \multirow[b]{2}{*}{ Population } & \multirow[b]{2}{*}{$\begin{array}{c}\text { Pop. } \\
\text { Total }\end{array}$} & \multirow[b]{2}{*}{ Model } & \multirow[b]{2}{*}{$\begin{array}{c}\text { Thick. } \\
\text { Spec } \\
\text { (mils) }\end{array}$} & \multicolumn{12}{|c|}{ Projected Proportion of Cylinders Below Thickness Criterion } \\
\hline & & & & $\begin{array}{l}20 \\
\text { Esti- } \\
\text { mate }\end{array}$ & $\begin{array}{l}03 \\
95 \% \\
\text { UCB }\end{array}$ & $\begin{array}{r}20 \\
\text { Esti- } \\
\text { mate }\end{array}$ & $\begin{array}{l}05 \\
95 \% \\
\text { UCB }\end{array}$ & $\begin{array}{l}20 \\
\text { Esti- } \\
\text { mate }\end{array}$ & $\begin{array}{l}10 \\
95 \% \\
\text { UCB }\end{array}$ & $\begin{array}{r}20 \\
\text { Esti- } \\
\text { mate }\end{array}$ & $\begin{array}{l}015 \\
95 \% \\
\text { UCB }\end{array}$ & $\begin{array}{l}20 \\
\text { Esti- } \\
\text { mate }\end{array}$ & $\begin{array}{l}20 \\
95 \% \\
\text { UCB }\end{array}$ & $\begin{array}{r}20 \\
\text { Esti- } \\
\text { mate }\end{array}$ & $\begin{array}{l}25 \\
95 \% \\
\text { UCB }\end{array}$ \\
\hline \multirow{3}{*}{ PORTS thin (P-scan data) } & 17,269 & Slope Fit & 250 & .0390 & .0585 & .0454 & .0672 & .0644 & .0917 & .0872 & .1195 & .1132 & .1503 & .1414 & .1839 \\
\hline & & & 62.5 & .0000 & .0000 & .0000 & .0000 & .0000 & .0000 & .0000 & .0000 & .0000 & .0000 & .0000 & .0000 \\
\hline & & & 0 & .0000 & .0000 & .0000 & .0000 & .0000 & .0000 & .0000 & .0000 & .0000 & .0000 & .0000 & .0000 \\
\hline \multirow[t]{3}{*}{ PORTS thin top } & 8,501 & Slope Fit & 250 & .0008 & .0053 & .0009 & .0057 & .0012 & .0065 & .0015 & .0074 & .0019 & .0084 & .0022 & .0095 \\
\hline & & & 62.5 & .0000 & .0000 & .0000 & .0000 & .0000 & .0000 & .0000 & .0000 & .0000 & .0000 & .0000 & .0000 \\
\hline & & & 0 & .0000 & .0000 & .0000 & .0000 & .0000 & .0000 & .0000 & .0000 & .0000 & .0000 & .0000 & .0000 \\
\hline \multirow[t]{3}{*}{ PORTS thin bottom } & 8,768 & Slope Fit & 250 & .0007 & .0049 & .0009 & .0053 & .0012 & .0065 & .0016 & .0077 & .0020 & .0090 & .0025 & .0104 \\
\hline & & & 62.5 & .0000 & .0000 & .0000 & .0000 & .0000 & .0000 & .0000 & .0000 & .0000 & .0000 & .0000 & .0000 \\
\hline & & & 0 & .0000 & .0000 & .0000 & .0000 & .0000 & .0000 & .0000 & .0000 & .0000 & .0000 & .0000 & .0000 \\
\hline \multirow[t]{3}{*}{ ETTP/PGDP/PORTS thick top } & 962 & Slope Fit & 500 & .0010 & .0030 & .0010 & .0031 & .0010 & .0032 & .0011 & .0034 & .0011 & .0036 & .0011 & .0038 \\
\hline & & & 62.5 & .0000 & .0000 & .0000 & .0000 & .0000 & .0000 & .0000 & .0000 & .0000 & .0000 & .0000 & .0000 \\
\hline & & & 0 & .0000 & .0000 & .0000 & .0000 & .0000 & .0000 & .0000 & .0000 & .0000 & .0000 & .0000 & .0000 \\
\hline \multirow[t]{3}{*}{ ETTP/PGDP/PORTS thick bottom } & 1,062 & Slope Fit & 500 & .0055 & .0129 & .0056 & .0131 & .0057 & .0136 & .0058 & .0142 & .0060 & .0147 & .0061 & .0153 \\
\hline & & & 62.5 & .0000 & .0000 & .0000 & .0000 & .0000 & .0000 & .0000 & .0000 & .0000 & .0000 & .0000 & .0000 \\
\hline & & & 0 & .0000 & .0000 & .0000 & .0000 & .0000 & .0000 & .0000 & .0000 & .0000 & .0000 & .0000 & .0000 \\
\hline \multirow[t]{3}{*}{ ETTP/PGDP/PORTS thin skirted top } & 2,285 & Slope $=1$ & 250 & .0088 & .0232 & .0103 & .0264 & .0149 & .0353 & .0206 & .0455 & .0273 & .0570 & .0352 & .0696 \\
\hline & & & 62.5 & .0000 & .0004 & .0000 & .0005 & .0001 & .0007 & .0001 & .0011 & .0002 & .0016 & .0004 & .0022 \\
\hline & & & 0 & .0000 & .0001 & .0000 & .0002 & .0000 & .0003 & .0000 & .0005 & .0001 & .0007 & .0001 & .0010 \\
\hline \multirow[t]{3}{*}{ ETTP/PGDP/PORTS thin skirted btm } & 2,487 & Slope $=1$ & 250 & .0337 & .0664 & .0375 & .0724 & .0480 & .0880 & .0595 & .1043 & .0720 & .1211 & .0852 & .1382 \\
\hline & & & 62.5 & .0013 & .0054 & .0015 & .0061 & .0021 & .0081 & .0029 & .0105 & .0039 & .0131 & .0051 & .0161 \\
\hline & & & 0 & .0006 & .0031 & .0007 & .0035 & .0010 & .0047 & .0015 & .0062 & .0020 & .0078 & .0026 & .0097 \\
\hline
\end{tabular}


Table 9C-cont'd. Indirect-Model Projections (Projected Proportions) for Target Years and Minimum Thickness Criteria

\begin{tabular}{|c|c|c|c|c|c|c|c|c|c|c|c|c|c|c|c|}
\hline \multirow[b]{2}{*}{ Population } & \multirow[b]{2}{*}{$\begin{array}{c}\text { Pop. } \\
\text { Total } \\
\end{array}$} & \multirow[b]{2}{*}{ Model } & \multirow[b]{2}{*}{$\begin{array}{l}\text { Thick. } \\
\text { Spec } \\
\text { (mils) }\end{array}$} & \multicolumn{12}{|c|}{ Projected Proportion of Cylinders Below Thickness Criterion } \\
\hline & & & & $\begin{array}{l}20 \\
\text { Esti- } \\
\text { mate }\end{array}$ & $\begin{array}{l}03 \\
95 \% \\
\text { UCB }\end{array}$ & $\begin{array}{r}20 \\
\text { Esti- } \\
\text { mate }\end{array}$ & $\begin{array}{l}05 \\
95 \% \\
\text { UCB }\end{array}$ & $\begin{array}{l}\text { 201 } \\
\text { Esti- } \\
\text { mate }\end{array}$ & $\begin{array}{l}10 \\
95 \% \\
\text { UCB }\end{array}$ & $\begin{array}{l}20 \\
\text { Esti- } \\
\text { mate }\end{array}$ & $\begin{array}{l}15 \\
95 \% \\
\text { UCB }\end{array}$ & $\begin{array}{l}20 \\
\text { Esti- } \\
\text { mate }\end{array}$ & $\begin{array}{l}20 \\
95 \% \\
\text { UCB }\end{array}$ & $\begin{array}{l}20 \\
\text { Esti- } \\
\text { mate }\end{array}$ & $\begin{array}{l}25 \\
95 \% \\
\text { UCB }\end{array}$ \\
\hline \multirow[t]{3}{*}{ ETTP/PGDP/PORTS thick skirted } & 2,024 & Slope $=1$ & 500 & .0073 & .0239 & .0084 & .0264 & .0114 & .0331 & .0149 & .0405 & .0189 & .0485 & .0235 & .0569 \\
\hline & & & 62.5 & .0001 & .0013 & .0002 & .0015 & .0002 & .0020 & .0004 & .0027 & .0005 & .0035 & .0007 & .0044 \\
\hline & & & 0 & .0001 & .0009 & .0001 & .0011 & .0002 & .0015 & .0002 & .0020 & .0004 & .0026 & .0005 & .0033 \\
\hline \multirow[t]{3}{*}{ ETTP/PGDP/PORTS 1/2" (30" dia.) top } & 1,317 & Slope $=1$ & 100 & .0026 & .0123 & .0030 & .0137 & .0042 & .0173 & .0056 & .0214 & .0073 & .0258 & .0093 & .0306 \\
\hline & & & 62.5 & .0019 & .0098 & .0022 & .0110 & .0031 & .0140 & .0042 & .0174 & .0055 & .0211 & .0071 & .0251 \\
\hline & & & 0 & .0012 & .0069 & .0014 & .0078 & .0020 & .0100 & .0027 & .0126 & .0036 & .0154 & .0046 & .0185 \\
\hline \multirow[t]{3}{*}{ ETTP/PGDP/PORTS 1/2" (30" dia.) btm } & 1,646 & Slope $=1$ & 100 & .0040 & .0179 & .0046 & .0198 & .0064 & .0250 & .0085 & .0306 & .0110 & .0367 & .0138 & .0432 \\
\hline & & & 62.5 & .0029 & .0144 & .0034 & .0160 & .0047 & .0203 & .0064 & .0250 & .0083 & .0302 & .0106 & .0358 \\
\hline & & & 0 & .0018 & .0102 & .0021 & .0114 & .0030 & .0146 & .0041 & .0183 & .0054 & .0222 & .0069 & .0266 \\
\hline
\end{tabular}


Table 10C. Direct-Model Projections (Projected Proportions) for Target Years and Thickness Criteria

\begin{tabular}{|c|c|c|c|c|c|c|c|c|}
\hline \multirow[b]{2}{*}{ Cylinder Grouping } & \multirow[b]{2}{*}{$\begin{array}{l}\text { Pop. } \\
\text { Total }\end{array}$} & \multirow[b]{2}{*}{$\begin{array}{c}\text { Thick. } \\
\text { Spec } \\
\text { (mils) }\end{array}$} & \multicolumn{6}{|c|}{ Projected Proportion of Cylinders Below Minimum Thickness Criteria } \\
\hline & & & $\begin{array}{l}2003 \\
\text { Esti- } \\
\text { mate }\end{array}$ & $\begin{array}{l}2005 \\
\text { Esti- } \\
\text { mate }\end{array}$ & $\begin{array}{l}2010 \\
\text { Esti- } \\
\text { mate }\end{array}$ & $\begin{array}{l}2015 \\
\text { Esti- } \\
\text { mate }\end{array}$ & $\begin{array}{l}2020 \\
\text { Esti- } \\
\text { mate }\end{array}$ & $\begin{array}{l}2025 \\
\text { Esti- } \\
\text { mate }\end{array}$ \\
\hline \multirow[t]{3}{*}{ ETTP thin (P-scan data) } & 4,037 & 250 & .3863 & .4033 & .4405 & .4710 & .4959 & .5165 \\
\hline & & 62.5 & .0036 & .0039 & .0046 & .0052 & .0057 & .0062 \\
\hline & & 0 & .0016 & .0017 & .0023 & .0026 & .0030 & .0033 \\
\hline \multirow[t]{3}{*}{ ETTP thin, K-yard bottom } & 1,167 & 250 & .0590 & .0616 & .0678 & .0740 & .0808 & .0881 \\
\hline & & 62.5 & .0027 & .0029 & .0031 & .0033 & .0036 & .0040 \\
\hline & & 0 & .0008 & .0009 & .0014 & .0018 & .0022 & .0027 \\
\hline \multirow[t]{3}{*}{ ETTP thin, except $\mathrm{K}$-yard bottom } & 2,870 & 250 & .0464 & .0494 & .0569 & .0639 & .0707 & .0773 \\
\hline & & 62.5 & .0016 & .0019 & .0023 & .0028 & .0032 & .0035 \\
\hline & & 0 & .0004 & .0006 & .0008 & .0011 & .0015 & .0019 \\
\hline \multirow[t]{3}{*}{ PGDP thin (P-scan data) } & 32,231 & 250 & .0603 & .0672 & .0854 & .1026 & .1195 & .1357 \\
\hline & & 62.5 & .0010 & .0012 & .0016 & .0022 & .0028 & .0034 \\
\hline & & 0 & .0004 & .0005 & .0006 & .0009 & .0012 & .0015 \\
\hline \multirow[t]{3}{*}{ PGDP thin bottom, former G-yard } & 1,983 & 250 & .0409 & .0432 & .0486 & .0536 & .0583 & .0627 \\
\hline & & 62.5 & .0019 & .0021 & .0025 & .0028 & .0031 & .0034 \\
\hline & & 0 & .0005 & .0006 & .0009 & .0012 & .0015 & .0019 \\
\hline \multirow[t]{3}{*}{ PGDP thin btm, excpt fmr G-yard } & 15,347 & 250 & .0171 & .0188 & .0232 & .0285 & .0335 & .0382 \\
\hline & & 62.5 & .0006 & .0007 & .0009 & .0012 & .0016 & .0021 \\
\hline & & 0 & .0002 & .0002 & .0003 & .0005 & .0005 & .0008 \\
\hline \multirow[t]{3}{*}{ PGDP thin top } & 14,901 & 250 & .0147 & .0161 & .0198 & .0238 & .0282 & .0323 \\
\hline & & 62.5 & .0006 & .0006 & .0009 & .0011 & .0015 & .0019 \\
\hline & & 0 & .0002 & .0002 & .0003 & .0004 & .0005 & .0007 \\
\hline
\end{tabular}


Table 10C-cont'd. Direct-Model Projections (Projected Proportions) for Target Years and Thickness Criteria

\begin{tabular}{|c|c|c|c|c|c|c|c|c|}
\hline \multirow[b]{2}{*}{ Cylinder Grouping } & \multirow[b]{2}{*}{$\begin{array}{l}\text { Pop. } \\
\text { Total } \\
\end{array}$} & \multirow[b]{2}{*}{$\begin{array}{l}\text { Thick. } \\
\text { Spec } \\
\text { (mils) }\end{array}$} & \multicolumn{6}{|c|}{ Projected Proportion of Cylinders Below Minimum Thickness Criteria } \\
\hline & & & $\begin{array}{l}2003 \\
\text { Esti- } \\
\text { mate }\end{array}$ & $\begin{array}{l}2005 \\
\text { Esti- } \\
\text { mate }\end{array}$ & $\begin{array}{l}2010 \\
\text { Esti- } \\
\text { mate }\end{array}$ & $\begin{array}{l}2015 \\
\text { Esti- } \\
\text { mate }\end{array}$ & $\begin{array}{l}2020 \\
\text { Esti- } \\
\text { mate }\end{array}$ & $\begin{array}{l}2025 \\
\text { Esti- } \\
\text { mate }\end{array}$ \\
\hline \multirow[t]{3}{*}{ PORTS thin (P-scan data) } & 17,269 & 250 & .0634 & .0708 & .0890 & .1062 & .1237 & .1390 \\
\hline & & 62.5 & .0011 & .0012 & .0017 & .0023 & .0029 & .0035 \\
\hline & & 0 & .0004 & .0005 & .0007 & .0009 & .0012 & .0015 \\
\hline \multirow[t]{3}{*}{ PORTS thin top } & 8,501 & 250 & .0120 & .0131 & .0160 & .0193 & .0228 & .0266 \\
\hline & & 62.5 & .0005 & .0006 & .0008 & .0011 & .0014 & .0018 \\
\hline & & 0 & .0002 & .0002 & .0003 & .0004 & .0005 & .0007 \\
\hline \multirow[t]{3}{*}{ PORTS thin bottom } & 8,768 & 250 & .0119 & .0131 & .0161 & .0194 & .0230 & .0269 \\
\hline & & 62.5 & .0005 & .0006 & .0008 & .0011 & .0014 & .0018 \\
\hline & & 0 & .0002 & .0002 & .0003 & .0004 & .0005 & .0007 \\
\hline \multirow[t]{3}{*}{ ETTP/PGDP/PORTS thick top } & 962 & 500 & .0135 & .0144 & .0169 & .0194 & .0215 & .0235 \\
\hline & & 62.5 & .0001 & .0001 & .0001 & .0001 & .0001 & .0001 \\
\hline & & 0 & .0001 & .0001 & .0001 & .0001 & .0001 & .0001 \\
\hline \multirow[t]{3}{*}{ ETTP/PGDP/PORTS thick bottom } & 1,062 & 500 & .0134 & .0143 & .0168 & .0193 & .0215 & .0234 \\
\hline & & 62.5 & .0001 & .0001 & .0001 & .0001 & .0001 & .0001 \\
\hline & & 0 & .0001 & .0001 & .0001 & .0001 & .0001 & .0001 \\
\hline \multirow[t]{3}{*}{ ETTP/PGDP/PORTS thin skirted top } & 2,285 & 250 & .0208 & .0218 & .0247 & .0280 & .0312 & .0339 \\
\hline & & 62.5 & .0015 & .0016 & .0022 & .0023 & .0027 & .0028 \\
\hline & & 0 & .0004 & .0004 & .0006 & .0009 & .0013 & .0015 \\
\hline \multirow[t]{3}{*}{ ETTP/PGDP/PORTS thin skirted btm } & 2,487 & 250 & .0249 & .0265 & .0303 & .0340 & .0376 & .0411 \\
\hline & & 62.5 & .0017 & .0020 & .0023 & .0025 & .0028 & .0030 \\
\hline & & 0 & .0004 & .0006 & .0008 & .0011 & .0014 & .0016 \\
\hline
\end{tabular}


Table 10C-cont'd. Direct-Model Projections (Projected Proportions) for Target Years and Thickness Criteria

\begin{tabular}{|c|c|c|c|c|c|c|c|c|}
\hline \multirow[b]{2}{*}{ Cylinder Grouping } & \multirow[b]{2}{*}{$\begin{array}{l}\text { Pop. } \\
\text { Total } \\
\end{array}$} & \multirow[b]{2}{*}{$\begin{array}{l}\text { Thick. } \\
\text { Spec } \\
\text { (mils) }\end{array}$} & \multicolumn{6}{|c|}{ Projected Proportion of Cylinders Below Minimum Thickness Criteria } \\
\hline & & & $\begin{array}{l}2003 \\
\text { Esti- } \\
\text { mate }\end{array}$ & $\begin{array}{l}2005 \\
\text { Esti- } \\
\text { mate }\end{array}$ & $\begin{array}{l}2010 \\
\text { Esti- } \\
\text { mate }\end{array}$ & $\begin{array}{l}2015 \\
\text { Esti- } \\
\text { mate }\end{array}$ & $\begin{array}{l}2020 \\
\text { Esti- } \\
\text { mate }\end{array}$ & $\begin{array}{l}2025 \\
\text { Esti- } \\
\text { mate }\end{array}$ \\
\hline \multirow{3}{*}{ ETTP/PGDP/PORTS thick skirted } & 2,024 & 500 & .0030 & .0031 & .0036 & .0040 & .0047 & .0053 \\
\hline & & 62.5 & .0001 & .0001 & .0001 & .0001 & .0001 & .0001 \\
\hline & & 0 & .0001 & .0001 & .0001 & .0001 & .0001 & .0001 \\
\hline \multirow[t]{3}{*}{ ETTP/PGDP/PORTS 1/2" (30" dia.) top } & 1,317 & 100 & .0004 & .0004 & .0004 & .0007 & .0010 & .0012 \\
\hline & & 62.5 & .0001 & .0001 & .0004 & .0004 & .0004 & .0006 \\
\hline & & 0 & .0001 & .0001 & .0001 & .0001 & .0001 & .0003 \\
\hline \multirow[t]{3}{*}{ ETTP/PGDP/PORTS 1/2" (30" dia.) btm } & 1,646 & 100 & .0004 & .0004 & .0005 & .0008 & .0011 & .0014 \\
\hline & & 62.5 & .0001 & .0002 & .0004 & .0004 & .0004 & .0007 \\
\hline & & 0 & .0001 & .0001 & .0001 & .0001 & .0002 & .0004 \\
\hline
\end{tabular}


Table 11. Cylinders Excluded from Tables 9B (Indirect Model) or 10B (Direct Model)

\begin{tabular}{|c|c|c|c|c|c|c|}
\hline Cylinder Grouping & $\begin{array}{c}\text { Max. } \\
\text { Pit } \\
\text { Depth }\end{array}$ & $\begin{array}{c}\text { Min. } \\
\text { Thick- } \\
\text { ness }\end{array}$ & $\begin{array}{c}\text { Year } \\
\text { Meas- } \\
\text { ured }\end{array}$ & $\begin{array}{c}\text { Measure- } \\
\text { ment } \\
\text { Method }\end{array}$ & Cylinder & $\begin{array}{c}\text { Model(s) } \\
\text { Excluded } \\
\text { For }\end{array}$ \\
\hline \multirow[t]{3}{*}{ ETTP thin (P-scan data) } & 180 & 140 & 1994 & P-Scan & 00734000 & Indirect \\
\hline & 315 & 0 & 1992 & Visual & 00795300 & Both \\
\hline & 315 & 0 & 1992 & Visual & 10124400 & Both \\
\hline \multirow[t]{2}{*}{ ETTP thin, except K-yard bottom } & 205 & 134 & 2001 & Man-UT & 01202700 & Both \\
\hline & 182 & 161 & 2001 & Man-UT & 11679700 & Both \\
\hline \multirow[t]{4}{*}{ PGDP thin btm, excpt fmr G-yard } & 217 & 124 & 2001 & Man-UT & 00553900 & Both \\
\hline & 208 & 141 & 2001 & Man-UT & 01036700 & Both \\
\hline & 240 & 124 & 2001 & Man-UT & 01374300 & Both \\
\hline & 172 & 186 & 2001 & Man-UT & 11290300 & Both \\
\hline PORTS thin bottom & & 183 & 2002 & Man-UT & 11431000 & Both \\
\hline ETTP/PGDP/PORTS thick bottom & 34 & 464 & 1998 & Man-UT & 00238000 & Direct \\
\hline \multirow[t]{10}{*}{ ETTP/PGDP/PORTS 1/2" (30" dia.) top } & 168 & 332 & 1999 & Man-UT & D2364600 & Indirect \\
\hline & 424 & 97 & 1999 & Man-UT & D2789500 & Both \\
\hline & 181 & 353 & 1999 & Man-UT & D3472600 & Indirect \\
\hline & 241 & 255 & 2002 & Man-UT & D3472900 & Both \\
\hline & 189 & 201 & 2001 & Man-UT & D3597000 & Both \\
\hline & 340 & 164 & 1999 & Man-UT & D3722700 & Both \\
\hline & 251 & 236 & 1999 & Man-UT & D3909600 & Both \\
\hline & 280 & 250 & 2001 & Man-UT & D5192900 & Both \\
\hline & 167 & 376 & 2001 & Man-UT & D7784700 & Indirect \\
\hline & 284 & 209 & 2001 & Man-UT & W0049200 & Both \\
\hline \multirow[t]{7}{*}{ ETTP/PGDP/PORTS 1/2" (30" dia.) btm } & 173 & 317 & 2002 & Man-UT & D3124900 & Both \\
\hline & 339 & 199 & 2001 & Man-UT & D3175600 & Both \\
\hline & 313 & 187 & 1999 & Man-UT & D3478700 & Both \\
\hline & 196 & 284 & 1999 & Man-UT & D3870400 & Both \\
\hline & 334 & 171 & 2001 & Man-UT & D3920100 & Both \\
\hline & 205 & 274 & 1999 & Man-UT & D3954600 & Both \\
\hline & 234 & 305 & 2002 & Man-UT & D4260300 & Both \\
\hline
\end{tabular}

Proportions Projections. The projections in Tables 9A and 10A can also be used on a relative basis, for example, to prioritize cylinder groups. Because biases, conservative or otherwise, tend to cancel out in comparisons, comparisons tend to be more robust than absolute estimates. For such comparisons, it is convenient to examine proportions of cylinders relative to cylinder count totals rather than the totals themselves. Tables 9C and 10C are the analogs of Tables 9A and 10A with proportions of cylinders rather than absolute counts. Disregarding the P-scan results, the tables show the cylinder groups where individual cylinders are projected most likely to fail either the breach or 62.5 mil criteria. In decreasing order, the first four groups for the indirect model (Table 9C) are ETTP thin-wall, K-yard bottom; ETTP thin-wall, except 
K-yard bottom; thin, skirted bottom cylinders; and PGDP former G-yard bottom cylinders. For the direct model (Table 10C), the first four groups are ETTP thin-wall, K-yard bottom; ETTP thin-wall, except Kyard bottom; PGDP former G-yard bottom; and thin, skirted bottom cylinders. Though the two tables differ in the proportions, the subpopulation risk rankings based on the proportions are about the same.

Projections by Cylinder Group and Age. Because the likelihood that a cylinder will fail a particular thickness criteria depends on both the cylinder's subpopulation and age, and because the cylinder subpopulations have different cylinder age distributions, focusing on subpopulations in general can be misleading. For example, the "average" cylinder in a large subpopulation composed of many new cylinders and a few very old ones may have only a tiny chance of failing a particular criteria, but the oldest cylinders in the same population might nevertheless be likely to fail. Therefore it is useful to examine cylinders grouped by both subpopulation and age.

Table 12 lists cylinder groups by both subpopulation and age. For each subpopulation and age group, the table lists the direct and indirect-model risk estimates expressed as percentages of cylinders expected to fail the 62.5 mil thickness criteria. The estimates are expressed as percentages (100 times the corresponding probability estimates) because the probabilities are very small numbers. The groups are listed in descending order of their direct-model risk percentage estimates.

Many of the age-subpopulation groups have the same estimate, .01506, of the percentage of noncompliant cylinders. The value .01506 is $100 /(2(\mathrm{~N}+1))$, where $\mathrm{N}=3,318$ is the number of observations in the directmodel regression. Without additional assumptions (as would be implied for example by a continuous distribution function), the value $1 /(2(\mathrm{~N}+1))$ is a lower limit on what a reasonable estimate of the failure percentage should be. This is discussed in Schmoyer (1992). Thus the direct model cannot resolve probabilities smaller than $1 /(2(3,318+1))=.0001506$. This lower bound also contributes to the directmodel's tendency to overestimate the numbers of failures of the breach or 62.5 mil criteria.

Table 12 shows that it is the combination of both age and subpopulation together that leads to the highest percentage risks. For example, the direct-model risk percentage estimate for the age-47 ETTP, thin-wall, Kyard bottom cylinders is $.295 \%$. That is, the probability that one of these cylinders will fail the 62.5 mil spec is estimates to be .00295 . The corresponding probability estimate for Portsmouth, thin-wall, bottom cylinders is .00219. However, the estimate in Table 9C of the overall probability of failing the 62.5 mil criterion is .0013 for the ETTP, thin-wall, K-yard bottom cylinders, while the corresponding estimate for Portsmouth, thin-wall, bottom cylinders is .0000. The age distribution charts in Figures 19 and 27 show that the ETTP, K-yard bottom cylinders are much older on average than the Portsmouth cylinders. But the age-specific failure rate estimate for the age-47 Portsmouth cylinders is nearly as high as the rate for the age-47 ETTP, K-yard bottom cylinders. The age-47 Portsmouth cylinders should not be discounted simply because they belong to a subpopulation of cylinders that is younger on the whole.

Table 12 is for the 62.5 mil thickness criterion, but tables for other criteria are similar. The direct and indirect models are evaluated and compared further in the next section. 
Table 12. Cylinder Population/Age Groups With Highest Estimated

Percentage Failing the 62.5 Mil Thickness Criteria

(By Descending Direct-Model Percentage Estimate)

\begin{tabular}{|c|c|c|c|c|c|}
\hline Cylinder Population & Age & $\mathbf{N}$ & $\begin{array}{c}\text { Ind. Model } \\
\text { Estimated } \\
\text { Percentage } \\
\text { Out of Spec* }\end{array}$ & $\begin{array}{l}\text { Ind. } \\
\text { Model } \\
\text { Rank }\end{array}$ & $\begin{array}{c}\text { Dir. Model } \\
\text { Estimated } \\
\text { Percentage } \\
\text { Out of Spec* }\end{array}$ \\
\hline ETTP thin, except $\mathrm{K}$-yard bottom & 47 & 37 & .45363 & 1 & .29771 \\
\hline ETTP thin, $\mathrm{K}$-yard bottom & 47 & 32 & .18651 & 10 & .29529 \\
\hline ETTP thin, except $\mathrm{K}$-yard bottom & 46 & 373 & .42394 & 2 & .28954 \\
\hline ETTP thin, $\mathrm{K}$-yard bottom & 46 & 357 & .17107 & 13 & .28784 \\
\hline ETTP thin, except $\mathrm{K}$-yard bottom & 44 & 45 & .36801 & 3 & .28626 \\
\hline ETTP thin, $\mathrm{K}$-yard bottom & 44 & 53 & .14277 & 15 & .28623 \\
\hline PGDP thin btm, excpt fmr G-yard & 47 & 280 & .02399 & 30 & .28623 \\
\hline ETTP thin, $\mathrm{K}$-yard bottom & 43 & 40 & .12988 & 16 & .28623 \\
\hline ETTP thin, except $\mathrm{K}$-yard bottom & 43 & 34 & .34177 & 4 & .28621 \\
\hline PGDP thin btm, excpt fmr G-yard & 46 & 30 & .02286 & 31 & .28575 \\
\hline PGDP thin bottom, former G-yard & 44 & 336 & .01017 & 44 & .28409 \\
\hline ETTP thin, $\mathrm{K}$-yard bottom & 41 & 168 & .10649 & 18 & .28148 \\
\hline ETTP thin, except $\mathrm{K}$-yard bottom & 41 & 168 & .29268 & 6 & .28121 \\
\hline PGDP thin bottom, former G-yard & 43 & 548 & .00965 & 45 & .27467 \\
\hline PGDP thin top & 47 & 257 & .00013 & 92 & .27234 \\
\hline ETTP thin, except $\mathrm{K}$-yard bottom & 40 & 472 & .26982 & 7 & .26472 \\
\hline ETTP thin, K-yard bottom & 40 & 482 & .09595 & 20 & .26227 \\
\hline PGDP thin btm, excpt fmr G-yard & 44 & 769 & .02067 & 32 & .25258 \\
\hline PGDP thin top & 46 & 36 & .00013 & 93 & .24919 \\
\hline ETTP thin, except K-yard bottom & 39 & 29 & .24806 & 8 & .23921 \\
\hline ETTP thin, K-yard bottom & 39 & 33 & .08614 & 22 & .23508 \\
\hline PGDP thin bottom, former G-yard & 41 & 250 & .00866 & 47 & .23083 \\
\hline PGDP thin btm, excpt fmr G-yard & 43 & 631 & .01962 & 33 & .22917 \\
\hline ETTP/PGDP/PORTS thin skirted btm & 47 & 456 & .17595 & 11 & .22899 \\
\hline PGDP thin top & 45 & 1 & .00012 & 94 & .22834 \\
\hline PORTS thin bottom & 47 & 134 & .00001 & 121 & .21882 \\
\hline PORTS thin top & 47 & 152 & .00001 & 124 & .21549 \\
\hline ETTP/PGDP/PORTS thin skirted btm & 46 & 1391 & .16260 & 14 & .21226 \\
\hline PGDP thin bottom, former G-yard & 40 & 138 & .00818 & 49 & .21177 \\
\hline
\end{tabular}

*Five significant digits are used here because of the wide range of the percentages. 
Table 12-cont'd. Cylinder Population/Age Groups

\begin{tabular}{|c|c|c|c|c|c|}
\hline Cylinder Population & Age & $\mathbf{N}$ & $\begin{array}{l}\text { Ind. Model } \\
\text { Estimated } \\
\text { Percentage } \\
\text { Out of Spec }\end{array}$ & $\begin{array}{l}\text { Ind. } \\
\text { Model } \\
\text { Rank }\end{array}$ & $\begin{array}{l}\text { Dir. Model } \\
\text { Estimated } \\
\text { Percentage } \\
\text { Out of Spec }\end{array}$ \\
\hline PGDP thin top & 44 & 364 & .00012 & 95 & .21132 \\
\hline PORTS thin bottom & 46 & 929 & .00001 & 123 & .20307 \\
\hline PORTS thin top & 46 & 911 & .00001 & 125 & .20097 \\
\hline PGDP thin bottom, former G-yard & 39 & 8 & .00772 & 52 & .19948 \\
\hline PGDP thin top & 43 & 985 & .00011 & 97 & .19857 \\
\hline ETTP/PGDP/PORTS thin skirted top & 47 & 436 & .00548 & 57 & .19829 \\
\hline PGDP thin btm, excpt fmr G-yard & 41 & 199 & .01759 & 34 & .19725 \\
\hline PGDP thin btm, excpt fmr G-yard & 40 & 227 & .01662 & 36 & .19584 \\
\hline ETTP/PGDP/PORTS thin skirted top & 46 & 1245 & .00480 & 61 & .19584 \\
\hline PORTS thin bottom & 44 & 41 & .00001 & 127 & .19565 \\
\hline PGDP thin btm, excpt fmr G-yard & 39 & 34 & .01568 & 38 & .19451 \\
\hline PGDP thin top & 41 & 626 & .00010 & 98 & .19428 \\
\hline PORTS thin bottom & 43 & 44 & .00001 & 128 & .19136 \\
\hline ETTP thin, except K-yard bottom & 35 & 10 & .17186 & 12 & .18944 \\
\hline PGDP thin top & 40 & 269 & .00010 & 99 & .18241 \\
\hline PGDP thin top & 39 & 45 & .00009 & 100 & .16805 \\
\hline PORTS thin bottom & 41 & 13 & .00001 & 133 & .16615 \\
\hline PORTS thin top & 41 & 163 & .00001 & 131 & .16544 \\
\hline PGDP thin bottom, former G-yard & 35 & 13 & .00600 & 55 & .16425 \\
\hline PORTS thin bottom & 40 & 7 & .00001 & 139 & .15834 \\
\hline PORTS thin top & 40 & 64 & .00001 & 137 & .15561 \\
\hline PORTS thin bottom & 39 & 7 & .00001 & 142 & .14089 \\
\hline PORTS thin top & 39 & 40 & .00001 & 141 & .13914 \\
\hline PGDP thin btm, excpt fmr G-yard & 35 & 162 & .01218 & 41 & .13791 \\
\hline PGDP thin btm, excpt fmr G-yard & 34 & 1 & .01138 & 43 & .13188 \\
\hline ETTP thin, except $\mathrm{K}$-yard bottom & 31 & 10 & .11219 & 17 & .13150 \\
\hline PGDP thin top & 35 & 143 & .00008 & 101 & .11994 \\
\hline ETTP thin, except K-yard bottom & 30 & 20 & .09971 & 19 & .11353 \\
\hline PGDP thin bottom, former G-yard & 31 & 13 & .00451 & 62 & .10727 \\
\hline ETTP thin, except K-yard bottom & 29 & 903 & .08816 & 21 & .10365 \\
\hline PORTS thin bottom & 35 & 8 & .00000 & 148 & .10186 \\
\hline
\end{tabular}




\section{Table 12-cont'd. Cylinder Population/Age Groups}

\begin{tabular}{|c|c|c|c|c|c|}
\hline Cylinder Population & Age & $\mathbf{N}$ & $\begin{array}{l}\text { Ind. Model } \\
\text { Estimated } \\
\text { Percentage } \\
\text { Out of Spec }\end{array}$ & $\begin{array}{l}\text { Ind. } \\
\text { Model } \\
\text { Rank }\end{array}$ & $\begin{array}{l}\text { Dir. Model } \\
\text { Estimated } \\
\text { Percentage } \\
\text { Out of Spec }\end{array}$ \\
\hline PORTS thin top & 35 & 50 & .00001 & 146 & .09996 \\
\hline PGDP thin bottom, former G-yard & 30 & 179 & .00417 & 64 & .09873 \\
\hline PGDP thin btm, excpt fmr G-yard & 31 & 27 & .00914 & 46 & .09148 \\
\hline PGDP thin bottom, former G-yard & 29 & 95 & .00384 & 66 & .08066 \\
\hline ETTP thin, except $\mathrm{K}$-yard bottom & 27 & 239 & .06773 & 23 & .07575 \\
\hline PGDP thin btm, excpt fmr G-yard & 30 & 308 & .00845 & 48 & .07548 \\
\hline PGDP thin top & 31 & 29 & .00006 & 102 & .07540 \\
\hline PGDP thin btm, excpt fmr G-yard & 29 & 141 & .00778 & 50 & .07519 \\
\hline PGDP thin top & 30 & 175 & .00006 & 103 & .07347 \\
\hline ETTP thin, except K-yard bottom & 26 & 316 & .05879 & 24 & .06811 \\
\hline PGDP thin bottom, former G-yard & 27 & 39 & .00323 & 68 & .06429 \\
\hline PORTS thin bottom & 31 & 133 & .00000 & 155 & .05456 \\
\hline PORTS thin top & 31 & 119 & .00000 & 152 & .05205 \\
\hline PGDP thin top & 29 & 87 & .00006 & 104 & .05197 \\
\hline ETTP thin, except $\mathrm{K}$-yard bottom & 25 & 175 & .05067 & 25 & .04816 \\
\hline PGDP thin bottom, former G-yard & 26 & 275 & .00295 & 70 & .04690 \\
\hline PORTS thin bottom & 30 & 529 & .00000 & 158 & .04528 \\
\hline PORTS thin top & 30 & 423 & .00000 & 153 & .04524 \\
\hline ETTP thin, except $\mathrm{K}$-yard bottom & 24 & 10 & .04333 & 26 & .04521 \\
\hline PGDP thin btm, excpt fmr G-yard & 27 & 185 & .00655 & 53 & .04521 \\
\hline PGDP thin bottom, former G-yard & 25 & 84 & .00268 & 71 & .04520 \\
\hline PORTS thin bottom & 29 & 522 & .00000 & 160 & .04519 \\
\hline PORTS thin top & 29 & 490 & .00000 & 154 & .04519 \\
\hline ETTP thin, except K-yard bottom & 23 & 17 & .03674 & 27 & .04519 \\
\hline PGDP thin top & 27 & 146 & .00005 & 106 & .04519 \\
\hline PGDP thin btm, excpt fmr G-yard & 26 & 1021 & .00597 & 56 & .04519 \\
\hline PORTS thin top & 28 & 1 & .00000 & 157 & .04519 \\
\hline PGDP thin btm, excpt fmr G-yard & 25 & 1234 & .00542 & 58 & .04519 \\
\hline PGDP thin btm, excpt fmr G-yard & 24 & 25 & .00490 & 60 & .04519 \\
\hline PGDP thin btm, excpt fmr G-yard & 23 & 331 & .00441 & 63 & .04519 \\
\hline PGDP thin top & 26 & 988 & .00005 & 107 & .04519 \\
\hline
\end{tabular}


Table 12-cont'd. Cylinder Population/Age Groups

\begin{tabular}{|c|c|c|c|c|c|}
\hline Cylinder Population & Age & $\mathbf{N}$ & $\begin{array}{l}\text { Ind. Model } \\
\text { Estimated } \\
\text { Percentage } \\
\text { Out of Spec }\end{array}$ & $\begin{array}{c}\text { Ind. } \\
\text { Model } \\
\text { Rank }\end{array}$ & $\begin{array}{l}\text { Dir. Model } \\
\text { Estimated } \\
\text { Percentage } \\
\text { Out of Spec }\end{array}$ \\
\hline PORTS thin bottom & 27 & 128 & .00000 & 163 & .04519 \\
\hline PGDP thin top & 25 & 1138 & .00004 & 108 & .04519 \\
\hline PORTS thin top & 27 & 127 & .00000 & 159 & .04519 \\
\hline PORTS thin top & 26 & 638 & .00000 & 161 & .04519 \\
\hline PORTS thin bottom & 26 & 772 & .00000 & 165 & .04519 \\
\hline PGDP thin top & 24 & 15 & .00004 & 109 & .04519 \\
\hline ETTP thin, except K-yard bottom & 22 & 3 & .03086 & 28 & .04519 \\
\hline PORTS thin bottom & 25 & 111 & .00000 & 167 & .04519 \\
\hline PORTS thin top & 25 & 112 & .00000 & 162 & .04519 \\
\hline PGDP thin btm, excpt fmr G-yard & 22 & 1255 & .00394 & 65 & .04519 \\
\hline ETTP thin, except $\mathrm{K}$-yard bottom & 21 & 6 & .02565 & 29 & .04519 \\
\hline PGDP thin top & 23 & 295 & .00004 & 110 & .04519 \\
\hline ETTP/PGDP/PORTS thin skirted btm & 24 & 446 & .01146 & 42 & .04511 \\
\hline PORTS thin bottom & 24 & 416 & .00000 & 170 & .04502 \\
\hline PORTS thin top & 24 & 400 & .00000 & 164 & .04483 \\
\hline PGDP thin top & 22 & 1113 & .00003 & 111 & .04104 \\
\hline PGDP thin btm, excpt fmr G-yard & 21 & 968 & .00351 & 67 & .03753 \\
\hline ETTP/PGDP/PORTS thin skirted top & 24 & 420 & .00005 & 105 & .03284 \\
\hline PORTS thin bottom & 23 & 17 & .00000 & 172 & .02314 \\
\hline PORTS thin top & 23 & 16 & .00000 & 166 & .02087 \\
\hline PGDP thin top & 21 & 906 & .00003 & 113 & .01534 \\
\hline ETTP/PGDP/PORTS thin skirted btm & 22 & 5 & .00773 & 51 & .01519 \\
\hline ETTP/PGDP/PORTS 1/2" (30" dia.) btm & 49 & 1601 & .29834 & 5 & .01509 \\
\hline PGDP thin btm, excpt fmr G-yard & 20 & 1 & .00310 & 69 & .01507 \\
\hline PORTS thin bottom & 22 & 280 & .00000 & 173 & .01507 \\
\hline PORTS thin top & 22 & 257 & .00000 & 168 & .01507 \\
\hline ETTP/PGDP/PORTS thin skirted top & 22 & 8 & .00003 & 114 & $.01506^{*}$ \\
\hline ETTP/PGDP/PORTS thin skirted btm & 21 & 185 & .00624 & 54 & .01506 \\
\hline ETTP/PGDP/PORTS 1/2" (30" dia.) top & 49 & 1267 & .19878 & 9 & .01506 \\
\hline PORTS thin bottom & 21 & 359 & .00000 & 176 & .01506 \\
\hline PORTS thin top & 21 & 343 & .00000 & 171 & .01506 \\
\hline
\end{tabular}

*Because of discreteness, $0.01506=[2(1+3,318)]^{-1}$ is the lower bound on the percentage (100 $\times$ probability) estimates for the direct-model regression method with 3,318 measured cylinders (see discussion in main text). 
Table 12-cont'd. Cylinder Population/Age Groups

\begin{tabular}{|c|c|c|c|c|c|}
\hline Cylinder Population & Age & $\mathbf{N}$ & $\begin{array}{l}\text { Ind. Model } \\
\text { Estimated } \\
\text { Percentage } \\
\text { Out of Spec }\end{array}$ & $\begin{array}{l}\text { Ind. } \\
\text { Model } \\
\text { Rank }\end{array}$ & $\begin{array}{l}\text { Dir. Model } \\
\text { Estimated } \\
\text { Percentage } \\
\text { Out of Spec }\end{array}$ \\
\hline ETTP/PGDP/PORTS thin skirted top & 21 & 175 & .00002 & 117 & .01506 \\
\hline PGDP thin btm, excpt fmr G-yard & 12 & 903 & .00081 & 80 & .01506 \\
\hline PGDP thin top & 9 & 417 & .00001 & 143 & .01506 \\
\hline ETTP/PGDP/PORTS thick skirted & 52 & 456 & .01724 & 35 & .01506 \\
\hline ETTP/PGDP/PORTS thick skirted & 51 & 473 & .01569 & 37 & .01506 \\
\hline ETTP/PGDP/PORTS thick skirted & 50 & 178 & .01425 & 39 & .01506 \\
\hline ETTP/PGDP/PORTS thick skirted & 49 & 644 & .01290 & 40 & .01506 \\
\hline ETTP/PGDP/PORTS thick skirted & 41 & 87 & .00525 & 59 & .01506 \\
\hline PGDP thin btm, excpt fmr G-yard & 18 & 55 & .00237 & 72 & .01506 \\
\hline ETTP thin, except K-yard bottom & 12 & 1 & .00231 & 73 & .01506 \\
\hline ETTP/PGDP/PORTS thin skirted btm & 17 & 1 & .00228 & 74 & .01506 \\
\hline PGDP thin btm, excpt fmr G-yard & 17 & 365 & .00204 & 75 & .01506 \\
\hline ETTP thin, except K-yard bottom & 11 & 1 & .00154 & 76 & .01506 \\
\hline PGDP thin btm, excpt fmr G-yard & 15 & 461 & .00147 & 77 & .01506 \\
\hline PGDP thin btm, excpt fmr G-yard & 14 & 1095 & .00123 & 78 & .01506 \\
\hline PGDP thin btm, excpt fmr G-yard & 13 & 639 & .00100 & 79 & .01506 \\
\hline PGDP thin btm, excpt fmr G-yard & 11 & 593 & .00064 & 81 & .01506 \\
\hline PGDP thin bottom, former G-yard & 14 & 1 & .00061 & 82 & .01506 \\
\hline PGDP thin btm, excpt fmr G-yard & 10 & 607 & .00049 & 83 & .01506 \\
\hline PGDP thin bottom, former G-yard & 12 & 3 & .00041 & 84 & .01506 \\
\hline PGDP thin btm, excpt fmr G-yard & 9 & 438 & .00037 & 85 & .01506 \\
\hline PGDP thin bottom, former G-yard & 11 & 1 & .00032 & 86 & .01506 \\
\hline ETTP/PGDP/PORTS thick skirted & 24 & 75 & .00026 & 87 & .01506 \\
\hline PGDP thin btm, excpt fmr G-yard & 8 & 1026 & .00026 & 88 & .01506 \\
\hline ETTP/PGDP/PORTS thick skirted & 23 & 41 & .00020 & 89 & .01506 \\
\hline ETTP thin, K-yard bottom & 12 & 2 & .00020 & 90 & .01506 \\
\hline PGDP thin btm, excpt fmr G-yard & 7 & 859 & .00018 & 91 & .01506 \\
\hline PGDP thin btm, excpt fmr G-yard & 6 & 409 & .00011 & 96 & .01506 \\
\hline PGDP thin btm, excpt fmr G-yard & 4 & 55 & .00003 & 112 & .01506 \\
\hline PGDP thin top & 18 & 64 & .00002 & 115 & .01506 \\
\hline PGDP thin top & 17 & 350 & .00002 & 116 & .01506 \\
\hline
\end{tabular}


Table 12-cont'd. Cylinder Population/Age Groups

\begin{tabular}{|c|c|c|c|c|c|}
\hline Cylinder Population & Age & $\mathbf{N}$ & $\begin{array}{l}\text { Ind. Model } \\
\text { Estimated } \\
\text { Percentage } \\
\text { Out of Spec }\end{array}$ & $\begin{array}{l}\text { Ind. } \\
\text { Model } \\
\text { Rank }\end{array}$ & $\begin{array}{l}\text { Dir. Model } \\
\text { Estimated } \\
\text { Percentage } \\
\text { Out of Spec }\end{array}$ \\
\hline PGDP thin top & 16 & 1 & .00002 & 118 & .01506 \\
\hline PGDP thin top & 15 & 452 & .00002 & 119 & .01506 \\
\hline PGDP thin top & 14 & 1042 & .00001 & 120 & .01506 \\
\hline PGDP thin top & 13 & 648 & .00001 & 122 & .01506 \\
\hline PGDP thin top & 12 & 822 & .00001 & 126 & .01506 \\
\hline PGDP thin top & 11 & 590 & .00001 & 129 & .01506 \\
\hline ETTP/PGDP/PORTS thick bottom & 52 & 232 & .00001 & 130 & .01506 \\
\hline ETTP/PGDP/PORTS thick bottom & 51 & 265 & .00001 & 132 & .01506 \\
\hline ETTP/PGDP/PORTS thick bottom & 50 & 95 & .00001 & 134 & .01506 \\
\hline ETTP/PGDP/PORTS thick skirted & 14 & 42 & .00001 & 135 & .01506 \\
\hline ETTP/PGDP/PORTS thick bottom & 49 & 324 & .00001 & 136 & .01506 \\
\hline PGDP thin top & 10 & 588 & .00001 & 138 & .01506 \\
\hline ETTP/PGDP/PORTS thick bottom & 41 & 48 & .00001 & 140 & .01506 \\
\hline ETTP/PGDP/PORTS thick bottom & 24 & 40 & .00001 & 144 & .01506 \\
\hline ETTP/PGDP/PORTS thick bottom & 23 & 20 & .00001 & 145 & .01506 \\
\hline PGDP thin top & 8 & 979 & .00001 & 147 & .01506 \\
\hline ETTP/PGDP/PORTS thick bottom & 14 & 23 & .00000 & 149 & .01506 \\
\hline PGDP thin top & 7 & 837 & .00000 & 150 & .01506 \\
\hline ETTP/PGDP/PORTS thick bottom & 10 & 3 & .00000 & 151 & .01506 \\
\hline PGDP thin top & 6 & 432 & .00000 & 156 & .01506 \\
\hline PGDP thin top & 4 & 56 & .00000 & 169 & .01506 \\
\hline ETTP/PGDP/PORTS thick skirted & 10 & 5 & .00000 & 174 & .01506 \\
\hline PORTS thin top & 18 & 348 & .00000 & 175 & .01506 \\
\hline PORTS thin top & 17 & 232 & .00000 & 177 & .01506 \\
\hline PORTS thin top & 16 & 88 & .00000 & 178 & .01506 \\
\hline PORTS thin top & 15 & 275 & .00000 & 179 & .01506 \\
\hline PORTS thin bottom & 18 & 363 & .00000 & 180 & .01506 \\
\hline PORTS thin top & 14 & 1293 & .00000 & 181 & .01506 \\
\hline PORTS thin bottom & 17 & 254 & .00000 & 182 & .01506 \\
\hline PORTS thin top & 13 & 607 & .00000 & 183 & .01506 \\
\hline PORTS thin bottom & 16 & 88 & .00000 & 184 & .01506 \\
\hline
\end{tabular}


Table 12-cont'd. Cylinder Population/Age Groups

\begin{tabular}{|c|c|c|c|c|c|}
\hline Cylinder Population & Age & $\mathbf{N}$ & $\begin{array}{l}\text { Ind. Model } \\
\text { Estimated } \\
\text { Percentage } \\
\text { Out of Spec }\end{array}$ & $\begin{array}{c}\text { Ind. } \\
\text { Model } \\
\text { Rank }\end{array}$ & $\begin{array}{l}\text { Dir. Model } \\
\text { Estimated } \\
\text { Percentage } \\
\text { Out of Spec }\end{array}$ \\
\hline PORTS thin top & 12 & 280 & .00000 & 185 & .01506 \\
\hline PORTS thin bottom & 15 & 269 & .00000 & 186 & .01506 \\
\hline PORTS thin top & 11 & 33 & .00000 & 187 & .01506 \\
\hline PORTS thin bottom & 14 & 1366 & .00000 & 188 & .01506 \\
\hline PORTS thin top & 10 & 148 & .00000 & 189 & .01506 \\
\hline PORTS thin bottom & 13 & 605 & .00000 & 190 & .01506 \\
\hline PORTS thin top & 9 & 430 & .00000 & 191 & .01506 \\
\hline PORTS thin bottom & 12 & 264 & .00000 & 192 & .01506 \\
\hline PORTS thin top & 8 & 110 & .00000 & 193 & .01506 \\
\hline PORTS thin bottom & 11 & 31 & .00000 & 194 & .01506 \\
\hline PORTS thin bottom & 10 & 157 & .00000 & 195 & .01506 \\
\hline PORTS thin top & 7 & 350 & .00000 & 196 & .01506 \\
\hline ETTP/PGDP/PORTS 1/2" (30" dia.) btm & 3 & 3 & .00000 & 197 & .01506 \\
\hline ETTP/PGDP/PORTS 1/2" (30" dia.) top & 3 & 1 & .00000 & 198 & .01506 \\
\hline PORTS thin bottom & 9 & 447 & .00000 & 199 & .01506 \\
\hline ETTP/PGDP/PORTS thick top & 52 & 224 & .00000 & 200 & .01506 \\
\hline ETTP/PGDP/PORTS thick top & 51 & 208 & .00000 & 201 & .01506 \\
\hline ETTP/PGDP/PORTS thick top & 50 & 83 & .00000 & 202 & .01506 \\
\hline ETTP/PGDP/PORTS thick top & 49 & 320 & .00000 & 203 & .01506 \\
\hline ETTP/PGDP/PORTS thick top & 41 & 39 & .00000 & 204 & .01506 \\
\hline PORTS thin bottom & 8 & 102 & .00000 & 205 & .01506 \\
\hline ETTP/PGDP/PORTS thick top & 24 & 35 & .00000 & 206 & .01506 \\
\hline ETTP/PGDP/PORTS thick top & 23 & 21 & .00000 & 207 & .01506 \\
\hline ETTP/PGDP/PORTS thick top & 14 & 19 & .00000 & 208 & .01506 \\
\hline PORTS thin bottom & 7 & 369 & .00000 & 209 & .01506 \\
\hline ETTP/PGDP/PORTS thick top & 10 & 2 & .00000 & 210 & .01506 \\
\hline
\end{tabular}




\section{MODEL EVALUATION AND COMPARISON}

In this section, the direct and indirect models are evaluated by comparing FY02 UT scan results with projections computed only with data obtained prior to FY02. This evaluation shows that the projected and observed results are consistent, though, except for the 250 or 500 mil thickness criteria, the evaluation is somewhat inconclusive, because the numbers of cylinders projected or observed to fail the lower thickness specifications in the relatively few scanned cylinders are essentially zero. Thus this section neither rejects nor confirms the suggestion in Section 5, based on judgment about cylinder populations, that the projections are conservative for the breach or 62.5 mil criteria.

Section 4 shows that the direct model leads to more sensible fits than the indirect model, because the indirect model slope estimate fails to stay within its 0-to-1 theoretical range for eight of seventeen subpopulations. Because the FY02 projections based on pre-FY02 data are similar for the direct and indirect models, the projections also do not provide much resolution between the two models. However, this section also introduces the Akaike information criterion (AIC, Akaike 1974), which helps to resolve further between the two models. The AIC suggests that the direct model is better.

\subsection{FY02 Projected vs Observed}

By fitting the indirect and direct models using only data collected prior to FY02, an assessment of the models can be made by comparing actual FY02 sampled results with model-based projections for FY02 that are entirely independent of the FY02 sampled results. This approach can be used both to compare the two modeling approaches and to assess the models on an absolute basis.

However, several factors complicate this evaluation. For example, the model-based minimum thickness projections are estimates, about which actual measured minimum thicknesses are expected to vary randomly. Another complication is the low probability of events like a thickness below 62.5 mils or a breach. It is low-probability events that we would most like to predict, yet only higher probability events, such as "thickness < 250 mils," are typically observed in samples. Although, both the direct and indirect models seem to perform reasonably well at forecasting numbers of cylinders with minimum thickness below 250 mils, 250 mils is much closer to the central part of the thickness distribution than 62.5 or 0 mils. Adequacy in forecasting numbers of cylinders with thickness below a value in the central part of a thickness distribution does not automatically imply adequacy in forecasting numbers of cylinders with thickness below a value in the lower tail of the distribution. The suggestion in the last section is that the model projections are in fact conservative for the lower thickness criteria.

For the various cylinder groups, Table 13 shows projected and FY02-observed numbers of cylinders with minimum thicknesses falling below 0 and 62.5 mils, and, for thin-wall, thick-wall, and $1 / 2$ "-thick cylinders, below 250, 500, and 100 mils respectively. The projections in Table 13 are for FY02, but they are computed only with data from FY01 and before. P-scan results are not considered at all in Table 13.

Overall, the direct model leads to a prediction that 11 cylinders would have fallen below the upper 250/500/100 mil criterion in FY02; the indirect model leads to a prediction of 10 cylinders. In the sample, 13 thin-wall cylinders were below the 250/500/100 mil criterion. Conclusions for the other thickness specifications are difficult to draw, because all of the projected cylinder counts are zeros, and so are all the observed counts. The predictions are consistent with the observed, but only in the sense that essentially none are predicted and none are observed. As discussed in the last section, when the projected numbers of breaches are scaled up to estimates for entire subpopulations rather than just those cylinders sampled, the projections seem somewhat high. 
Table 13. FY02 Indirect and Direct-Model Projected and Observed Counts for Sampled Cylinders

\begin{tabular}{|c|c|c|c|c|c|c|}
\hline Cylinder Population & $\begin{array}{c}\text { Number } \\
\text { in } \\
\text { Population }\end{array}$ & $\begin{array}{c}\text { Number } \\
\text { Sampled } \\
\text { from Pop. }\end{array}$ & $\begin{array}{c}\text { Thick. } \\
\text { Spec }\end{array}$ & $\begin{array}{c}\text { Observed } \\
\text { Number } \\
\text { Out of Spec }\end{array}$ & $\begin{array}{c}\text { Indirect Model } \\
\text { Projected } \\
\text { Number } \\
\text { Out of Spec }\end{array}$ & $\begin{array}{l}\text { Direct Model } \\
\text { Projected } \\
\text { Number } \\
\text { Out of Spec }\end{array}$ \\
\hline \multirow{3}{*}{ ETTP thin, K-yard bottom } & 1,167 & 60 & 250 & 6 & 4 & 3 \\
\hline & & & 62.5 & 0 & 0 & 0 \\
\hline & & & 0 & 0 & 0 & 0 \\
\hline \multirow[t]{3}{*}{ ETTP thin, except K-yard bottom } & 2,869 & 43 & 250 & 3 & 3 & 2 \\
\hline & & & 62.5 & 0 & 0 & 0 \\
\hline & & & 0 & 0 & 0 & 0 \\
\hline \multirow[t]{3}{*}{ PGDP thin bottom, former G-yard } & 1,983 & 15 & 250 & 1 & 0 & 1 \\
\hline & & & 62.5 & 0 & 0 & 0 \\
\hline & & & 0 & 0 & 0 & 0 \\
\hline \multirow[t]{3}{*}{ PGDP thin btm, excpt fmr G-yard } & 15,334 & 39 & 250 & 1 & 0 & 1 \\
\hline & & & 62.5 & 0 & 0 & 0 \\
\hline & & & 0 & 0 & 0 & 0 \\
\hline \multirow[t]{3}{*}{ PGDP thin top } & 14,896 & 23 & 250 & 1 & 0 & 0 \\
\hline & & & 62.5 & 0 & 0 & 0 \\
\hline & & & 0 & 0 & 0 & 0 \\
\hline \multirow[t]{3}{*}{ PORTS thin top } & 8,500 & 63 & 250 & 0 & 0 & 1 \\
\hline & & & 62.5 & 0 & 0 & 0 \\
\hline & & & 0 & 0 & 0 & 0 \\
\hline \multirow[t]{3}{*}{ PORTS thin bottom } & 8,765 & 54 & 250 & 1 & 0 & 1 \\
\hline & & & 62.5 & 0 & 0 & 0 \\
\hline & & & 0 & 0 & 0 & 0 \\
\hline
\end{tabular}


Table 13-con't. FY02 Indirect and Direct-Model Projected and Observed Counts for Sampled Cylinders

\begin{tabular}{|c|c|c|c|c|c|c|}
\hline Cylinder Population & $\begin{array}{c}\text { Number } \\
\text { in } \\
\text { Population }\end{array}$ & $\begin{array}{c}\text { Number } \\
\text { Sampled } \\
\text { from Pop. }\end{array}$ & $\begin{array}{l}\text { Thick. } \\
\text { Spec }\end{array}$ & $\begin{array}{c}\text { Observed } \\
\text { Number } \\
\text { Out of Spec }\end{array}$ & $\begin{array}{l}\text { Indirect Model } \\
\text { Projected } \\
\text { Number } \\
\text { Out of Spec }\end{array}$ & $\begin{array}{l}\text { Direct Model } \\
\text { Projected } \\
\text { Number } \\
\text { Out of Spec }\end{array}$ \\
\hline \multirow[t]{3}{*}{ ETTP/PGDP/PORTS thick top } & 951 & 18 & 500 & 0 & 0 & 0 \\
\hline & & & 62.5 & 0 & 0 & 0 \\
\hline & & & 0 & 0 & 0 & 0 \\
\hline \multirow[t]{3}{*}{ ETTP/PGDP/PORTS thick bottom } & 1,050 & 18 & 500 & 0 & 0 & 0 \\
\hline & & & 62.5 & 0 & 0 & 0 \\
\hline & & & 0 & 0 & 0 & 0 \\
\hline \multirow[t]{3}{*}{ ETTP/PGDP/PORTS thin skirted top } & 2,284 & 42 & 250 & 0 & 1 & 1 \\
\hline & & & 62.5 & 0 & 0 & 0 \\
\hline & & & 0 & 0 & 0 & 0 \\
\hline \multirow[t]{3}{*}{ ETTP/PGDP/PORTS thin skirted btm } & 2,484 & 33 & 250 & 0 & 2 & 1 \\
\hline & & & 62.5 & 0 & 0 & 0 \\
\hline & & & 0 & 0 & 0 & 0 \\
\hline \multirow[t]{3}{*}{ ETTP/PGDP/PORTS thick skirted } & 2,001 & 36 & 500 & 0 & 0 & 0 \\
\hline & & & 62.5 & 0 & 0 & 0 \\
\hline & & & 0 & 0 & 0 & 0 \\
\hline \multirow[t]{3}{*}{ ETTP/PGDP/PORTS 1/2" (30" dia.) top } & 1,268 & 11 & 100 & 0 & 0 & 0 \\
\hline & & & 62.5 & 0 & 0 & 0 \\
\hline & & & 0 & 0 & 0 & 0 \\
\hline \multirow[t]{3}{*}{ ETTP/PGDP/PORTS 1/2" (30" dia.) btm } & 1,604 & 14 & 100 & 0 & 0 & 0 \\
\hline & & & 62.5 & 0 & 0 & 0 \\
\hline & & & 0 & 0 & 0 & 0 \\
\hline
\end{tabular}




\subsection{Comparison by Akaike Information Criterion}

Comparing the direct and indirect models is difficult because the numbers of parameters in the two models differ substantially. For the seventeen cylinder groups, the direct model has nineteen parameters, including the standard deviation. The indirect model has, $3 \times 17=51$ parameters, including seventeen standard deviations, but not including parameters for the initial thickness distribution. Increasing the number of parameters in a model automatically improves model fitting criteria (e.g., the sum of squared regression residuals), which measure departures between the model-fitted and observed data. However, having more parameters does not automatically imply that a model will provide better projections of future measurements. (Otherwise arbitrarily high-order polynomials could be used to predict anything.) Increasing the number of parameters can in fact make future projections worse, because they become more susceptible to statistical error in the data.

The same logic applies whether the model fit criteria is based on sums of squared residuals or differences between observed and projected numbers below various thickness criteria. When the same data is used both to fit models and to evaluate their performance, comparisons of the models should be adjusted to account for differences in numbers of parameters. However, how to make such an adjustment is not an easy question. In this section, one such adjustment is considered. The point is not to consider the details of the adjustment itself, but rather simply to illustrate that after accounting for one such adjustment, the direct model does seem better than the indirect model.

Akaike (1974) considered the problem of comparing models with different numbers of parameters and developed a basis for model comparisons that has become known as the Akaike information criterion (AIC). The AIC is defined as

$$
\text { AIC }=-2(\log \text {-likelihood })+2 \text { (number of model parameters }),
$$

where "log-likelihood" denotes the maximized log-likelihood, and the method of maximum likelihood is the statistical method for estimating the parameters. The lower the AIC, the better the model fit. The second term in the AIC incorporates a penalty proportional to the number of model parameters, because having more parameters reduces the log-likelihood but does not necessarily improve model-based predictions of new measurements.

For a given thickness criterion $C$, and for any minimum thickness measurement $\mathrm{y}$, let the indicator function $I_{C}$ be defined as

$$
I_{C}(\mathrm{y})= \begin{cases}1 & \text { if } \mathrm{y} \leq C \\ 0 & \text { if } \mathrm{y}>C\end{cases}
$$

For each measured cylinder $I$ with minimum thickness measurement $\mathrm{y}_{\mathrm{i}}, I_{C}\left(\mathrm{y}_{\mathrm{i}}\right)$ is 1 if $\mathrm{y}_{\mathrm{i}}$ is at or below the thickness criterion $C$, and $I_{C}\left(\mathrm{y}_{\mathrm{i}}\right)$ is 0 otherwise. For each cylinder $i$, let $\mathrm{p}_{i}$ denote the probability, under either the direct or indirect model, that the minimum thickness is below $C$. Then for all measured cylinders, the probability of the observed number of cylinders with minimum thickness below $C$ is

$$
\prod_{\text {All cylinders } i} \mathrm{p}_{i}^{I_{C}\left(\mathrm{y}_{\mathrm{i}}\right)}\left(1-\mathrm{p}_{i}\right)^{1-I_{C}\left(\mathrm{y}_{\mathrm{i}}\right)}
$$

Under either the direct or indirect (or other) model, each $\mathrm{p}_{i}$ can be estimated using the model's parameter 
estimates. Let $\hat{\mathrm{p}}_{i}$ denote such an estimate. If the model parameters are estimated by the method of maximum likelihood, then for all of the measured cylinders, the likelihood of the observed number with minimum thickness below $C$ is the probability of the observed number with minimum thickness evaluated at the $\hat{\mathrm{p}}_{i}$, and the log-likelihood is thus

$$
\sum_{\text {All cylinders } i} I_{C}\left(\mathrm{y}_{\mathrm{i}}\right) \log \left(\hat{p}_{i}\right)+\left(1-I_{C}\left(\mathrm{y}_{\mathrm{i}}\right)\right) \log \left(1-\hat{\mathrm{p}}_{i}\right) .
$$

The indirect and direct model parameter estimation by least squares regression is not necessarily the same as maximum likelihood estimation, but the two estimation approaches lead to similar estimates. Thus the loglikelihoods can be evaluated approximately by plugging in the direct or indirect-model estimates.

Table 14 shows the direct and indirect-model approximate log-likelihood and AIC criteria for the 0, 62.5, and 250 mil criteria:

Table 14. Values of the Log-likelihood and Akaike Information Criterion (AIC)

\begin{tabular}{crrcr}
\hline $\begin{array}{c}\text { Thickness } \\
\text { Criterion }\end{array}$ & Log-likelihood & AIC & Log-likelihood & AIC \\
\hline 0 & -19.93 & 141.86 & -23.31 & 84.62 \\
62.5 & -16.20 & 135.50 & -22.87 & 83.74 \\
250 & $-13,133.57$ & $32,339.32$ & -632.78 & $1,303.56$ \\
\hline
\end{tabular}

Table 14 shows that even without imposing the AIC penalty for the number of model parameters, the loglikelihood for the direct model is greater (less negative) than the log-likelihood for the indirect model, for the 250 mil criteria. For the 0 and 62.5 mil criteria, the log-likelihood is greater for the indirect model, but by an amount that is small relative to the AIC adjustment. With the adjustment, the AIC is substantially smaller for the direct model. Although the likelihood is approximated here, these results suggest that the direct model is better, in this adjusted sense. 


\section{LIMITATIONS, CONCLUSIONS, AND RECOMMENDATIONS}

Recap. $\mathrm{UF}_{6}$ storage cylinder corrosion models are developed in this report for projecting numbers of cylinders expected to fail various thickness criteria. Data collected during FY02 is combined with previously collected UT data to update cylinder corrosion models. The CID is used to update cylinder subpopulation counts and to discount cylinders painted during the last ten years from cylinders assumed, in the calculations, to be at risk of continuing degradation because of corrosion.

Two different corrosion modeling approaches are considered. An indirect model relates maximum pit depths to cylinder age and subpopulation. The maximum pit depths are not measured directly, but rather are estimated as differences between maximum and minimum measured wall thicknesses. In order to estimate minimum wall thicknesses, the maximum pit depth model is combined with an initial thickness model using mathematics that assumes statistical independence of the distributions of the initial thicknesses and maximum pit depths. The independence assumption could fail, for example if steel quality and the initial thickness are correlated. The indirect model is then used to compute point estimates of the numbers of noncompliant cylinders. UCLs corresponding to the point estimates are also developed, but they seem too conservative to be useful.

A second corrosion modeling approach is based on a direct model that relates measured minimum wall thickness directly to cylinder age, subpopulation, and initial thickness estimates. The initial thickness estimates are incorporated into the minimum thickness model, and the assumption that initial thickness and pit depth are statistically independent is avoided. The direct-model approach also avoids problems with maximum pit depth estimates, which require good measurements of wall thicknesses maxima measured at relatively uncorroded areas of cylinder surfaces, assumed to be as new. The direct model admits better incorporation of the information that there is zero corrosion at age zero; the indirect model does not make good use of this information, because, in the indirect model, pit depths are lognormally distributed and zerodepth pits are inadmissable on the log scale. The direct model is also used to calculate projections of the numbers of noncompliant cylinders. The direct-model projections do account for statistical error in the regression parameter estimates and should be somewhat conservative in that respect.

In the indirect model, maximum pit depths are related to age by a power-law-if the fitted power-law slope is between 0 and 1 . If the fitted slope is not between 0 and 1, then the slope is set to 1 , and the model in which corrosion increases linearly in time is used instead. For the data and eight of the seventeen cylinder subpopulations considered in this report, the power-law does not fit, and the slope-set-to-one model is used instead. The failure of the power-law model is due at least in part to limitations of the cylinder thickness data. For various reasons, including a tendency in inspections to focus on deficient rather than good cylinder wall areas, minimum and maximum wall thickness measurements have sometimes been incompatible, and the power-law has not fit the maximum pit depth data very well. These difficulties with the indirect model were the primary reason for considering the direct model.

With the exception of some of the Portsmouth cylinders, the sampling process is cross-sectional: each scanned cylinder is scanned during only one fiscal year. Both the direct and indirect corrosion models suggest the ETTP thin-wall cylinders, both K-yard bottom and the remainder, are the most likely to fail various thickness specifications. The next most vulnerable groups are the thin, skirted bottom cylinders and PGDP former G-yard bottom cylinders.

Projections based only on measurements made before FY02 suggest that projections for upper thickness criteria $(250,500$, or 100 mils respectively for thin-wall, thick-wall, or $1 / 2$ " cylinders) are approximately correct. For both the direct and indirect models, projections of number of cylinders that fail the 0 (breach) or 62.5 mil criteria seem too conservative. However, the projected counts for the 0 and 62.5 mil criteria are substantially reduced if only a small percentage of the UT measurements are dropped from the 
analysis. This suggests that the cylinders corresponding to these measurements should be reevaluated to either confirm or reject the previous results.

Longitudinal-Study Possible Alternative. A possible alternative to cross-sectional monitoring would be longitudinal monitoring, with cylinders measured multiple times over the years. A randomly selected sample of cylinders measured repeatedly over the years could serve as bellwethers for all of the cylinders. Because each cylinder in such a sample could serve as its own control, changes in the sample could be measured more precisely than in cross-sectional samples. Similarly, scanning the same cylinders would compensate for measurement bias.

However, there are disadvantages to a longitudinal approach: (1) Though the approach would compensate for biases in the measurements that are consistent from year to year, it would not compensate for biases due to changes in the measurement method such as changes in instrument calibration or the change from Pscanning to manual UT scanning. ${ }^{12}$ (2) Because corrosion is a very slow process, it would take a long time to acquire enough longitudinal data to model corrosion effectively. The great majority of UT data already collected has been sampled on a cross-sectional basis and is needed to support near-term decisions about cylinder movements and dispositions. (On the other hand, many Portsmouth cylinders measured FY95-02 have been measured during more than one year. Those cylinder scan results could be incorporated into a longitudinal model.) (3) Finally, though an express objective in the cylinder monitoring is characterization, inspection is also a goal. Because year-to-year changes are so small, there is little point, from the perspective of inspection, in re-scanning the same cylinders. Measuring the same cylinders year after year diverts resources that could be used to scan cylinders that were not scanned previously. In a cross-sectional approach, new cylinders are scanned and thus inspected each year.

For these reasons, the cross-sectional monitoring approach seems preferable.

Limitations. Projecting cylinder conditions into the future on the basis of data collected with different goals, sampling schemes, and measurement methods is a difficult task, the limitations of which should be understood. Because it is less flexible and data anomalies do not affect it as easily, the direct corrosion model seems to fit the cylinder thickness data better than the indirect model. Yet while less flexibility is an advantage in dealing with noisy or anomalous data, it can be a disadvantage in reflecting the underlying physics of the corrosion process. And although the direct model seems to fit the cylinder data better, projections based on the two models are similar. Thus, there does not yet seem to be ample evidence to support the choice of either corrosion model over the other.

For both the direct and indirect approaches, relative to the variability of the data, corrosion appears to be only weakly related to cylinder age. That cylinder-to-cylinder variability is substantial, even for cylinders of the same age and grouping, is obvious from Figures 1-34. Nevertheless, age has an important and statistically significant effect on the corrosion process, and the oldest cylinders are of greatest concern. Tables 9A and 10A of subpopulation-wide numbers of cylinders projected to fall below the various thickness criteria can be misleading if careful attention is not also paid to the oldest and most vulnerable cylinders in each cylinder subpopulation. Previous editions of this report have not focused on age-specific projections, but attention should nevertheless also be paid to projections by both age and subpopulation, for example, as in Table 12.

\footnotetext{
${ }^{12}$ The cross-sectional approach does not compensate for changes in the measurement method either.
} 
The following caveats and limitations should be kept in mind when considering this report:

- Implicit in either the direct or indirect models is an assumption of age invariance-that newer or older cylinders alike had similar corrosion when they were the same age. The distributions of pit depths or wall thicknesses for 10 year old cylinders in a given population are assumed to be the same no matter when the cylinders were measured.

- Storage (e.g., ground contact) conditions have changed for many cylinders.

- Environmental changes such as acid rain are not accounted for.

- Cylinder sampling was not always random.

- Literature data for the atmospheric corrosion of steel might not apply to cylinder corrosion modeling, for example because of the thermal inertia of the cylinders.

- In the indirect model, the maximum pit depth data are only estimates, because initial wall thicknesses are estimates from maximum wall thicknesses.

- Age and population-specific projections should be considered in addition to projections by populations for all ages

Conclusions and Recommendations. Corrosion projections made in this report are based on analyses that account for cylinder subpopulations and ages. A myriad of other variables are not accounted for, however. Examples include how many use cycles the cylinders went through, how many nicks and scrapes, and the nature of former surface coatings, now perhaps long gone. There are variations in how the UT or P-scan measurements were made. The subpopulations themselves are only known approximately and in a few cases even the ages are approximate.

Because of extraneous sources of variation and other approximations, corrosion physics is blurred in the statistical noise. Thickness measurements vary widely about their model-based predictions. In this context, because there is not a definitive model choice based on corrosion physics, it does not make sense to try to resolve fine differences between either the deterministic or stochastic components of plausible corrosion models. The choice is not going to be clear. It is better to focus on general model behavior and on data quality and quantity, so that laws-of-large numbers will allow a general corrosion signal to be resolved from the statistical noise.

A indirect-model refinement that nevertheless might be considered would be the extreme value (statistical) distribution. (The direct model approach considered here is nonparametric - not based on any parametric distribution - though direct minimum thickness models could also be formulated with extreme-value error distributions.) The extreme value distribution has a physical basis for models of minima or maxima, and might provide an alternative to the indirect model that does not fail to conform with the power-law in so many cases. The failure of the power-law model in many cases might be due to improperly weighting the data in the model fitting, and the weighting is a reflection of the underlying statistical distribution (e.g., lognormal) that the indirect model assumes.

The following are the main recommendations of this report:

- The projections in this report are based on the assumption that historical trends will continue. However, many of the yards are being improved. Cylinders are being painted. When such changes can be quantified and accounted for, future analyses should incorporate them through adjustments to subpopulation definitions. 
- As it appears unlikely that apparent discrepancies between P-scan and manual UT results will ever be resolved, and as many new manual UT measurements have been made in recent years, P-scan data should be deprecated in favor of the manual UT data that is superseding it.

- Although the direct model appears to fit the UT cylinder thickness data better than the indirect model, that conclusion is still tentative. Until a clearer picture is established, cylinder thickness data should be analyzed using both the direct and indirect model approaches.

- Cylinders with manual UT scans identified in this report as outliers (Table 11) substantially influence the corrosion projections. These cylinders should be measured again to confirm or correct their thickness measurements. Alternatives to the outlier criteria used for this report (maximum pit depth $>160$ mils for the indirect model, regression residual $<-20$ mils for the direct model) should also be explored. 


\section{REFERENCES}

Akaike, H. (1974), "A New Look at Statistical Model Identification," IEEE Transactions on Automatic Control, 19, 716-723.

American National Standards for Nuclear Materials-Uranium Hexafluoride-Packaging for Transport, ANSI N14.1, American National Standards Institute, New York, 1995 edition.

Barber, E. J., J. H. DeVan, J. M. Googin, and M. S. Taylor (1994). Investigation of Breached Depleted UF ${ }_{6}$ Cylinders at the K-25 Site. ORNL/TM-12840, Oak Ridge, Tennessee, October 1994.

Blue, S. C. (1994). Facsimile from S.C. Blue to M. Taylor, October 27, 1994.

Blue, S. C. (1995). Memorandum from S.C. Blue to A.K. Balding, September 25, 1995.

Burden, R. L. and J. D. Faires (1989). Numerical Analysis, Fourth Edition, PWS-Kent Publishing Company, Boston.

David, H. A. (1981) Order Statistics, Second Edition, John Wiley \& Sons, New York.

Draper, N., and Smith, H. (1981) Applied Regression Analysis, 2nd ed., New York, John Wiley \& Sons.

DNFSB (1995) "Integrity of Uranium Hexafluoride Cylinders," Defense Nuclear Facilities Safety Board, DNFSB/TECH-4, May 5, 1995 (http://www.dnfsb.gov/techrpts/tech-4.html).

Felieu, S., M. Morcillo and S. Felieu, Jr. (1993a). The Prediction of Atmospheric Corrosion from Meteorological and Pollution Parameters--Ii. Annual Corrosion in Corrosion Science, Vol. 34 , No.3, pp.403-414.

Felieu, S., M. Morcillo and S. Felieu, Jr. (1993b). The Prediction of Atmospheric Corrosion from Meteorological and Pollution Parameters--II. Long-term Forecasts in Corrosion Science, Vol. 34, No.3, pp.415-422.

Horton, J. B. (1964). The Composition, Structure and Growth of the Atmospheric Rust on Various Steels. Thesis Lehigh University 1964, Bethlehem, PA.

Johnson, N. L., and Kotz, S. (1970) Continuous Univariate Distributions-1, Houghton Mifflin Co., Boston.

Legault, R. A. and G. Preban (1975). Corrosion-NACE, Vol. 31, p.117.

Lockheed Martin Energy Systems, Inc. (LMES) (1997a). UF $F_{6}$ Cylinder Project System Requirements Document (SRD). K/TSO-001, Rev.3. May 1997. U.S. Department of Energy.

Lockheed Martin Energy Systems, Inc. (LMES) (1997b). UF ${ }_{6}$ Cylinder Project System Engineering Management Plan (SEMP). K/TSO-017. July 1997. U.S. Department of Energy.

Lykins, M. L. and S. J. Pawel (1997). Evaluation of Wall Thickness in the Cylinder Head/Skirt Interface on Skirted Cylinders. Memorandum from M.L Lykins and S.J. Pawel to M.S. Taylor, ORNL/CST-SP-102297-1, October 22, 1997. 
Lyon, B. F. (1995). Prediction of External Corrosion for UF ${ }_{6}$ Cylinders: Results of an Empirical Method, ORNL/TM-13012. June 1995. Oak Ridge National Laboratory, Oak Ridge, Tennessee.

Lyon, B. F. (1996). Prediction of External Corrosion for Steel Cylinders at the Paducah Gaseous Diffusion Plant: Application of an Empirical Method, ORNL/TM-13192. February 1996. Oak Ridge National Laboratory, Oak Ridge, Tennessee.

Lyon, B. F. and M. L. Lykins (1996). Ultrasonic Thickness Sampling Plan for the Depleted Uranium Hexafluoride Program, ORNL/TM-13280. July 1996. Oak Ridge National Laboratory, Oak Ridge, Tennessee.

Lyon, B. F. (1997). Prediction of External Corrosion for Steel Cylinders, ORNL/TM-13359. February 1997. Oak Ridge National Laboratory, Oak Ridge, Tennessee.

Lyon, B. F. (1298). Prediction of External Corrosion for Steel Cylinders - 1998 Report, ORNL/TM13568. February 1998. Oak Ridge National Laboratory, Oak Ridge, Tennessee.

Lyon, B. F. (2000). Prediction of External Corrosion for Steel Cylinders - 2000 Report, ORNL/TM2000/96. June 2000. Oak Ridge National Laboratory, Oak Ridge, Tennessee.

Mughabghab, S. F. and T. M. Sullivan (1989). Evaluation of the Pitting Corrosion of Carbon Steels and Other Ferrous Metals in Soil Systems. Waste Management, Vol. 9, pp. 239-251.

Philpot, H. E. (1995). Memorandum from Halen Philpot to Valerie Newman, February 17, 1995.

Pourbaix, M. (1982). The Linear Bilogarithmic Law for Atmospheric Corrosion, in Atmospheric Corrosion (W. H. Ailor, ed.). John Wiley and Sons, New York.

Romanoff, M. (1957). Underground Corrosion. NBS Circular 579, National Bureau of Standards, Washington, D.C.

Rosen, R. S. and R. E. Glaser (1995). Letter to C.E. Bradley from R.S. Rosen and R.E. Glaser, November $8,1995$.

Rosen, R. S. and R. E. Glaser (1996). Letter to B.F. Lyon from R.S. Rosen and R.E. Glaser, April, 10, 1996.

Schmidt, M. A., J. K. Harper, and J. A. Broders (1996). A Comparison of Wall Thickness Measurements on $U F_{6}$ Cylinders using Scanning vs Hand-held Ultrasonic Probes. K/TSO-019, November 1996. Oak Ridge National Laboratory, Oak Ridge, Tennessee.

Schmoyer, R. L. (1992), "Asymptotically Valid Prediction Intervals for Linear Models," Technometrics, 34, 399-408.

Schmoyer, R. L., and Lyon, B. F. (2001). Prediction of External Corrosion for Steel Cylinders - 2001 Report, ORNL/TM-2001/164. September 2001. Oak Ridge National Laboratory, Oak Ridge, Tennessee.

Schmoyer, R. L., and Lyon, B. F. (2002). Prediction of External Corrosion for Steel Cylinders - 2002 Report, ORNL/TM-2002/143. July 2002. Oak Ridge National Laboratory, Oak Ridge, Tennessee. 


\section{APPENDIX A: FIGURES}




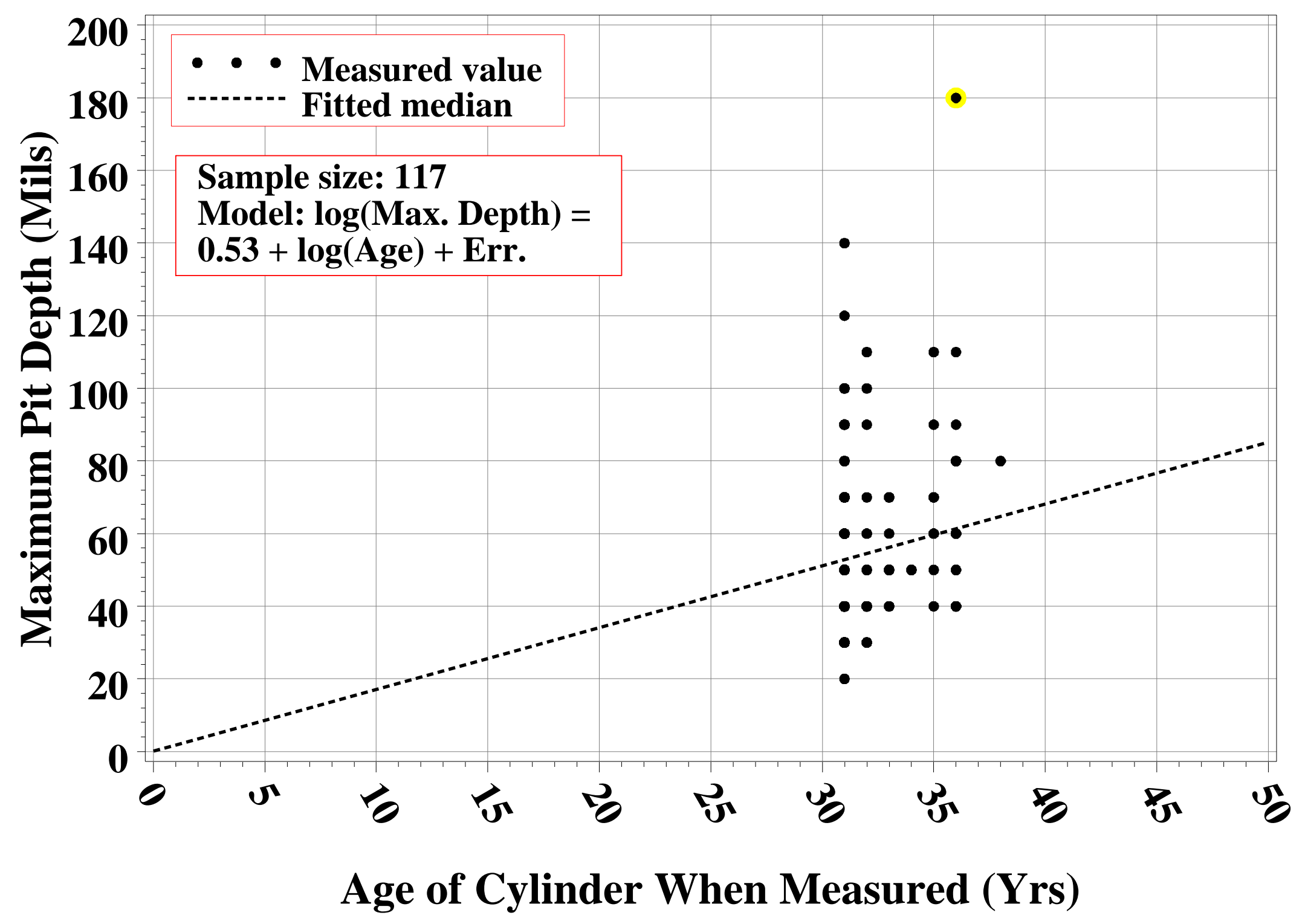

Figure 1. Maximum pit depth estimates for ETTP thin (P-scan data) cylinders. In Figures 1-17, points above 160 mils are highlighted (yellow), and those points are excluded from Table 9B. 


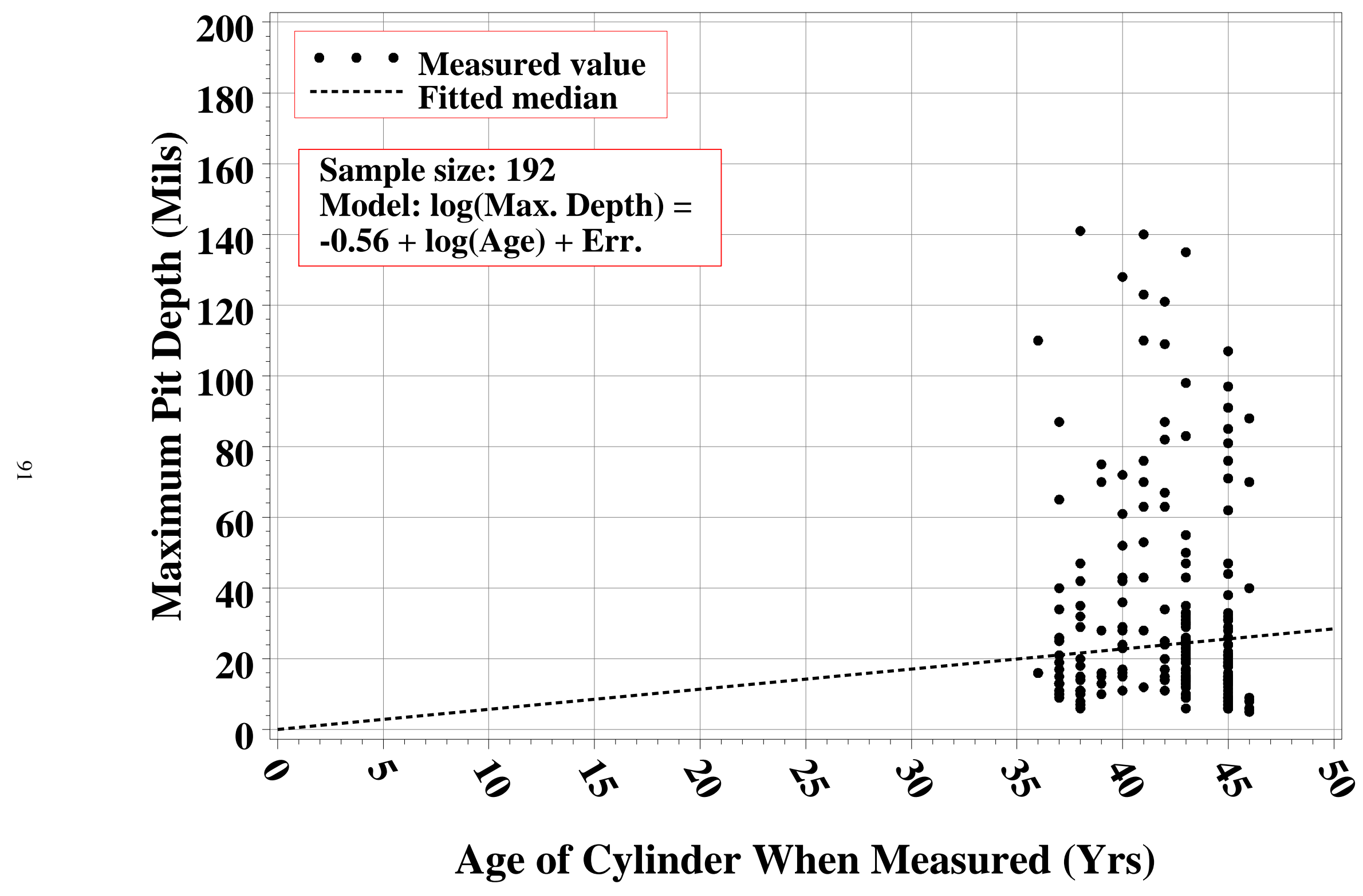

Figure 2. Maximum pit depth estimates for ETTP thin, K-yard bottom cylinders. 


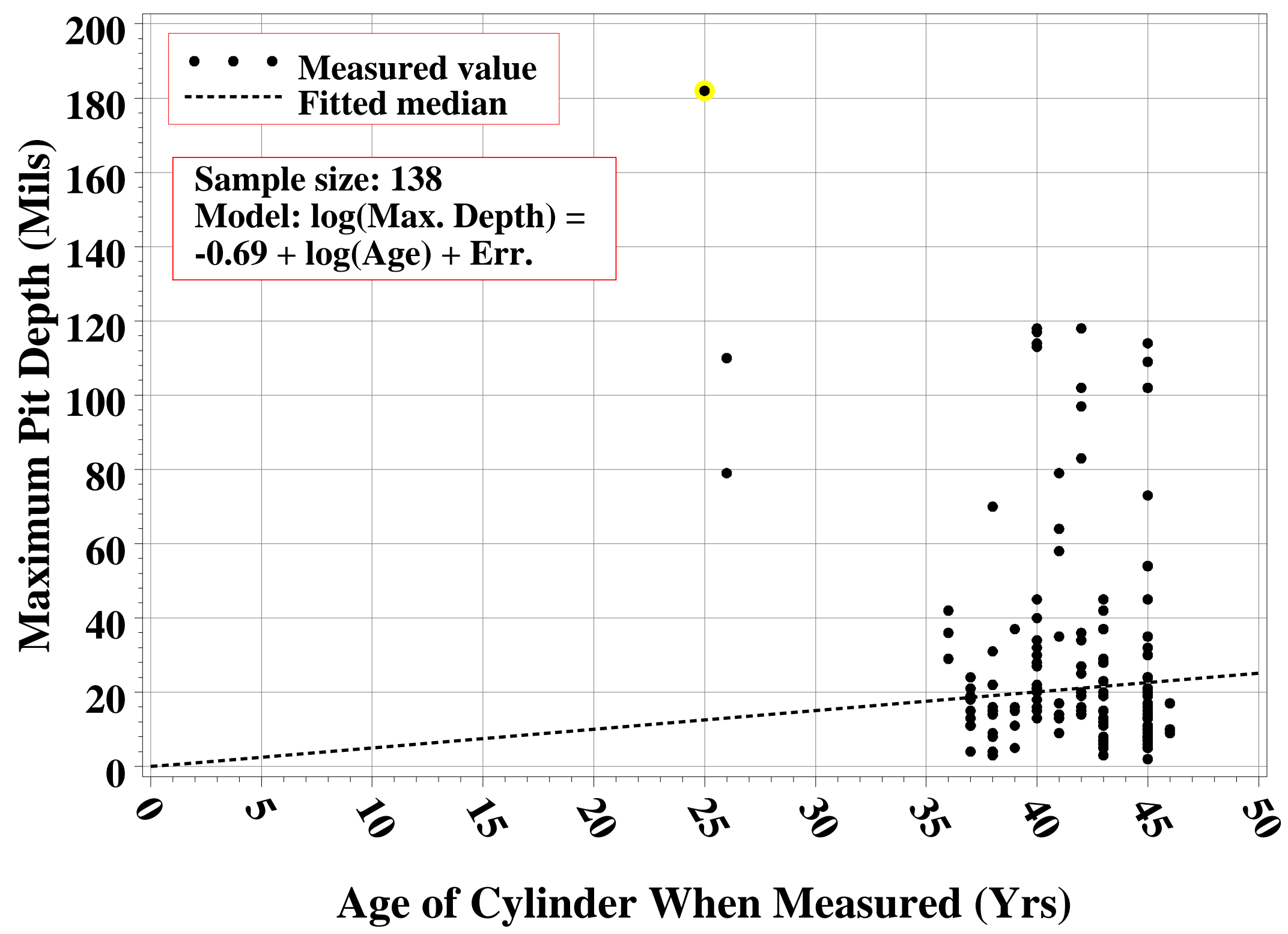

Figure 3. Maximum pit depth estimates for ETTP thin, except K-yard bottom cylinders. 


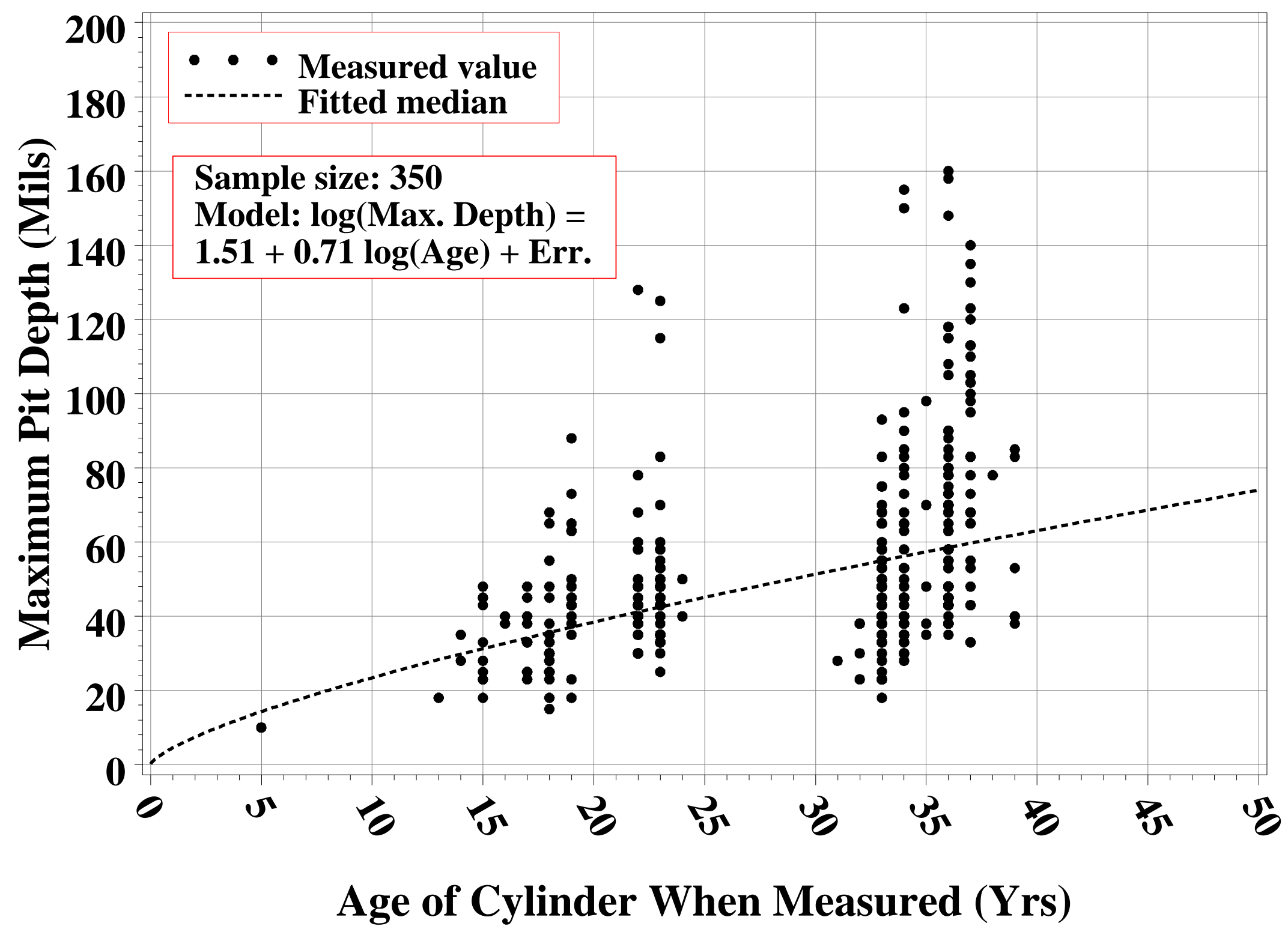

Figure 4. Maximum pit depth estimates for PGDP thin (P-scan data) cylinders. 


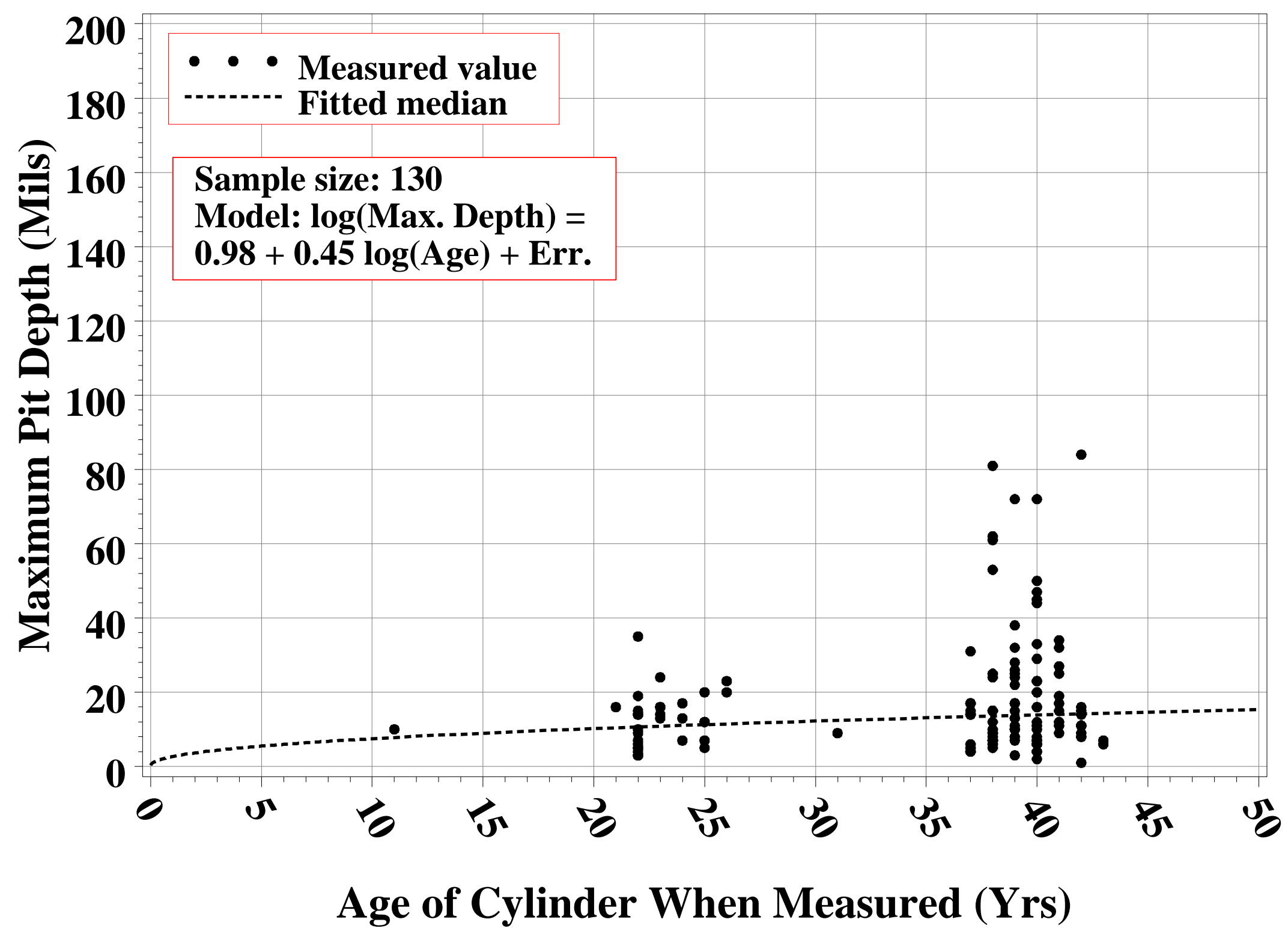

Figure 5. Maximum pit depth estimates for PGDP thin bottom, former G-yard cylinders. 


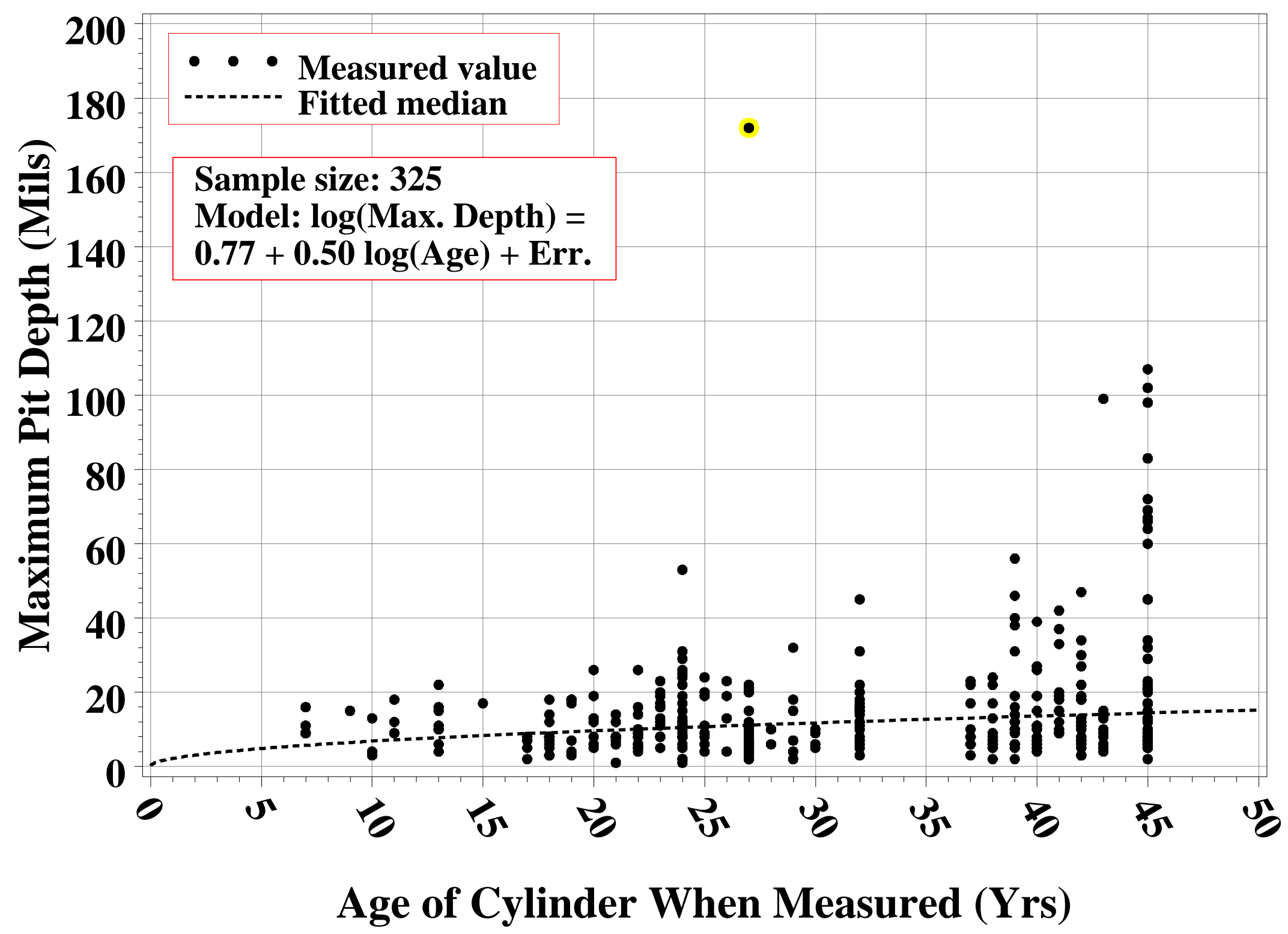

Figure 6. Maximum pit depth estimates for PGDP thin btm, excpt fmr G-yard cylinders. 


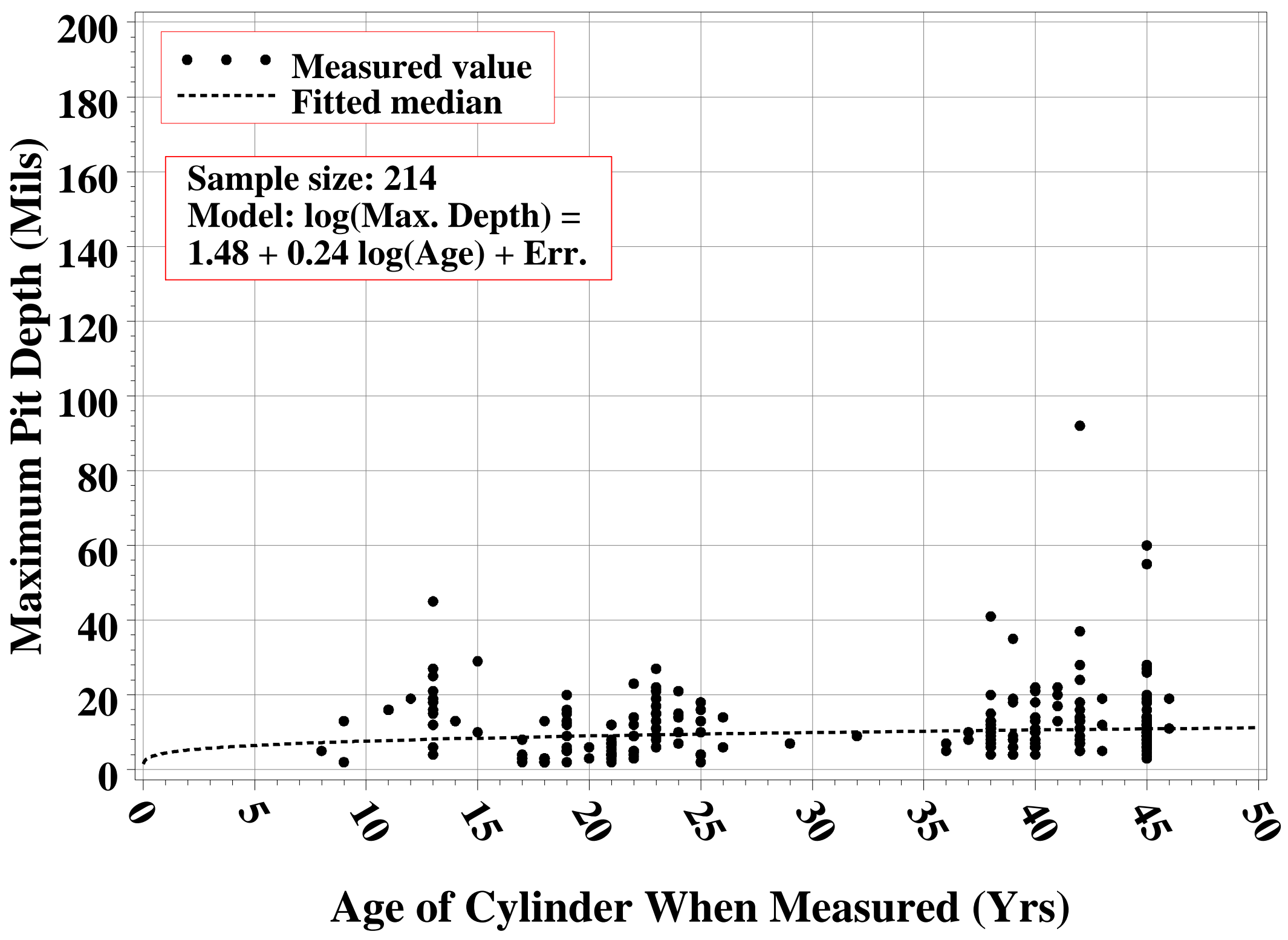

Figure 7. Maximum pit depth estimates for PGDP thin top cylinders. 


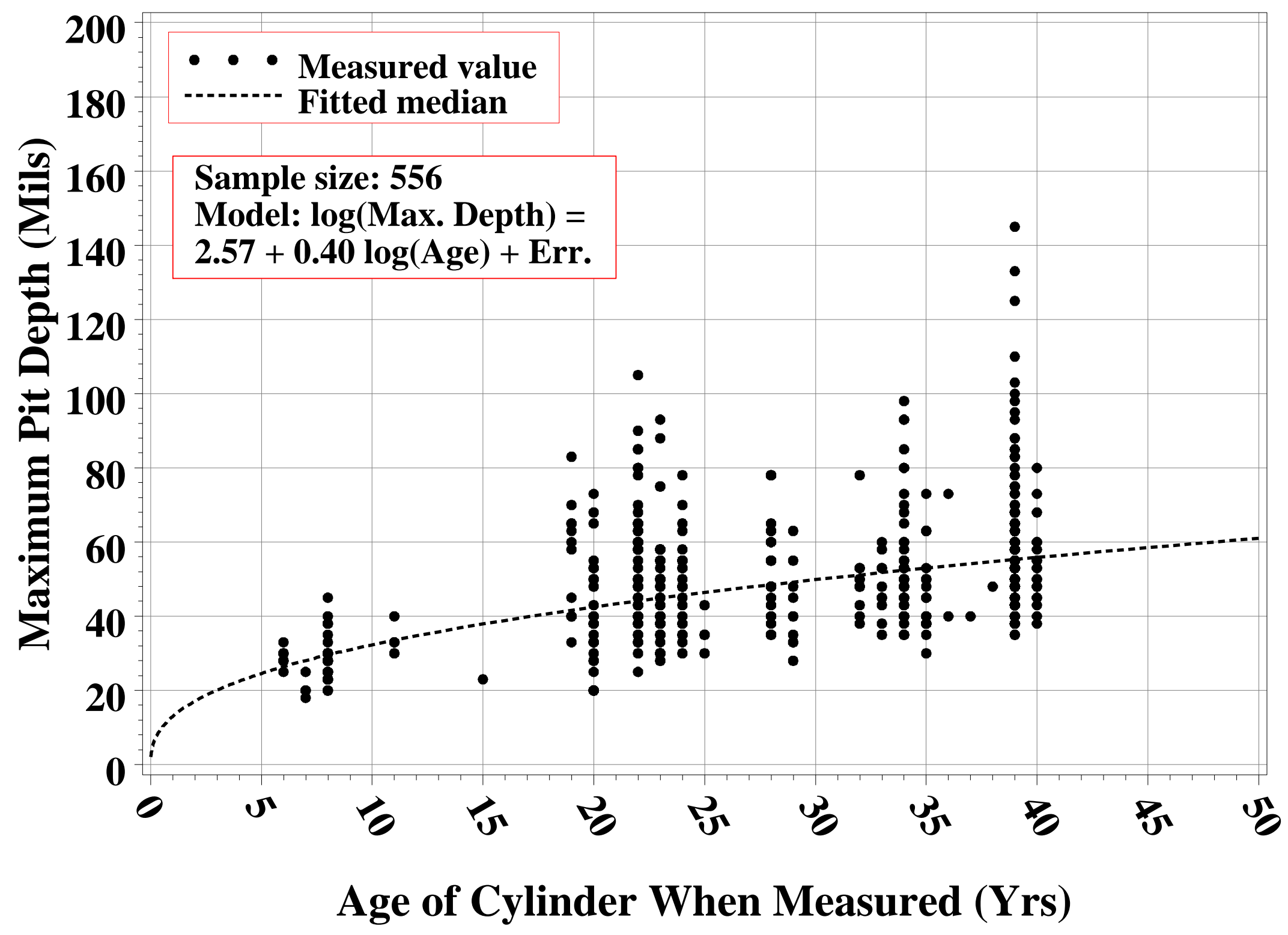

Figure 8. Maximum pit depth estimates for PORTS thin (P-scan data) cylinders. 


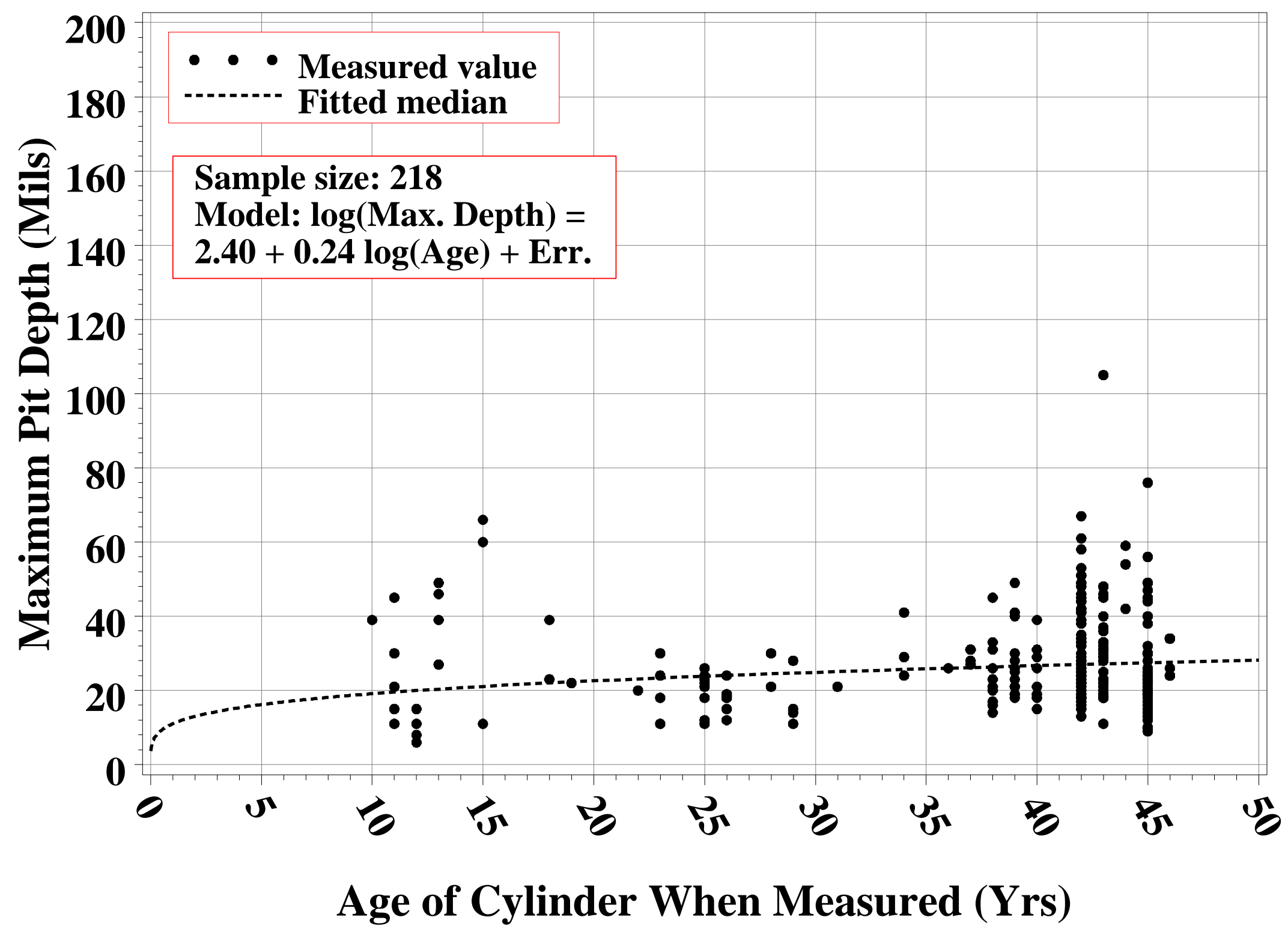

Figure 9. Maximum pit depth estimates for PORTS thin top cylinders. 


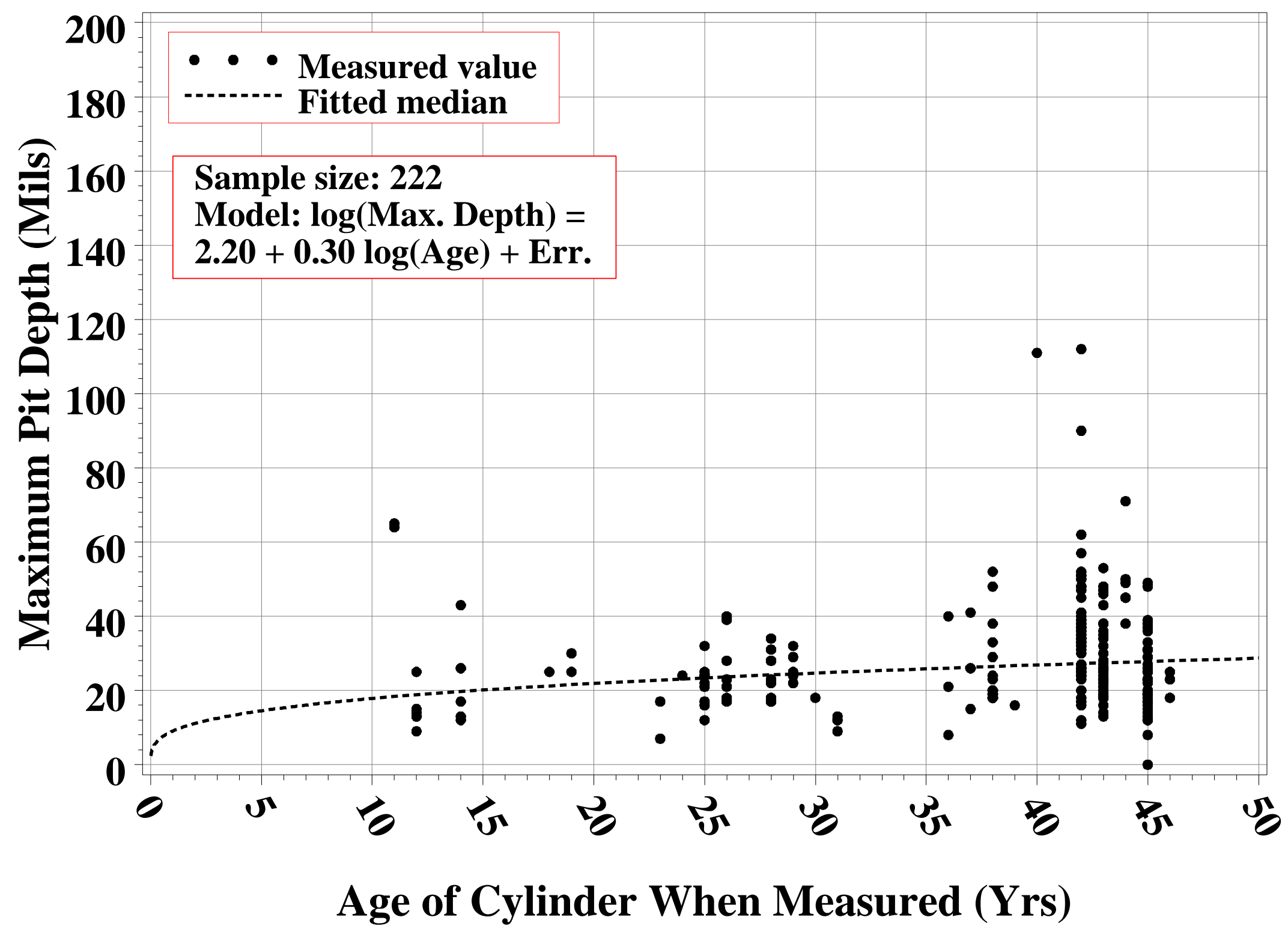

Figure 10. Maximum pit depth estimates for PORTS thin bottom cylinders. 


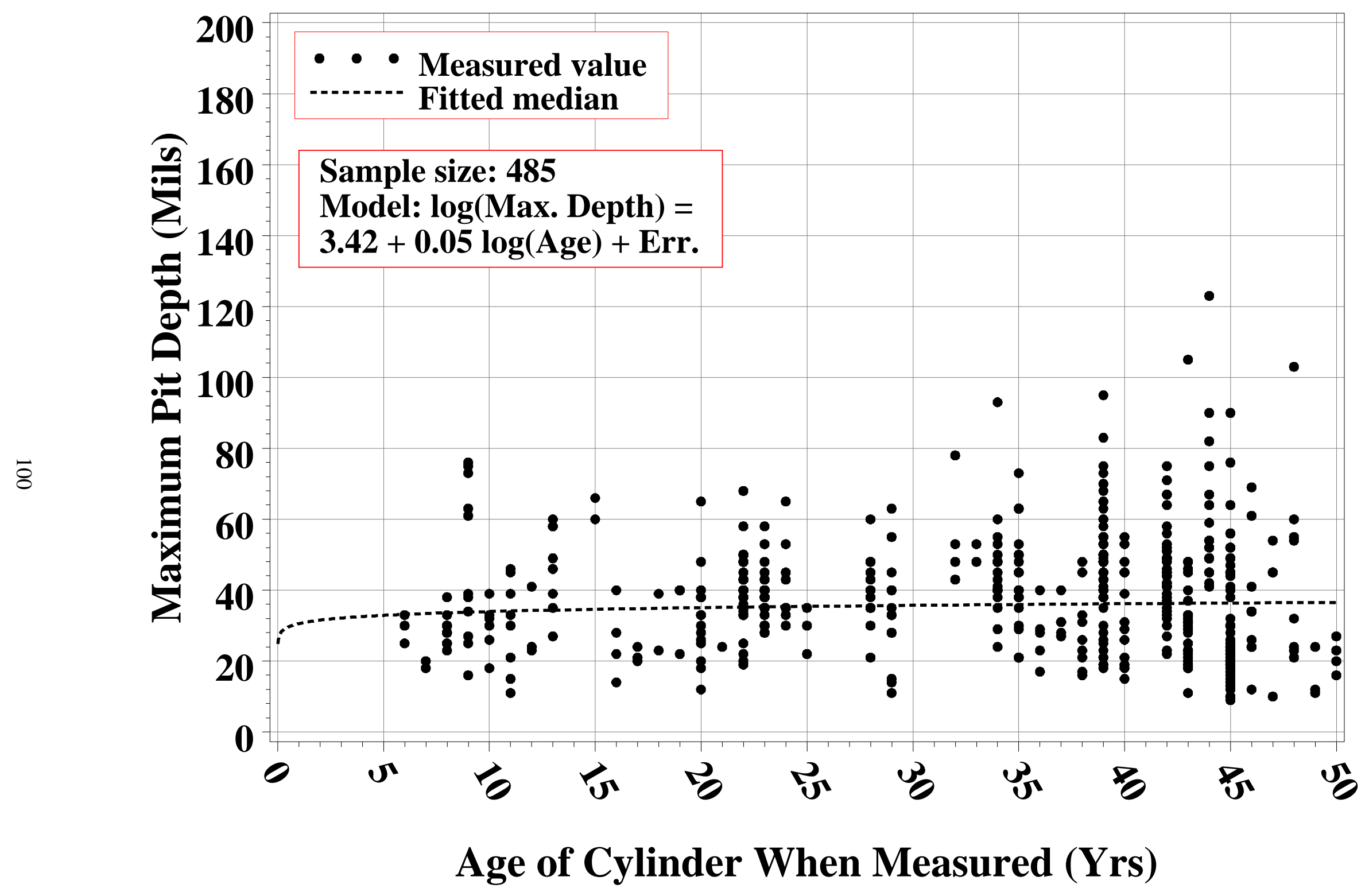

Figure 11. Maximum pit depth estimates for ETTP/PGDP/PORTS thick top cylinders. 


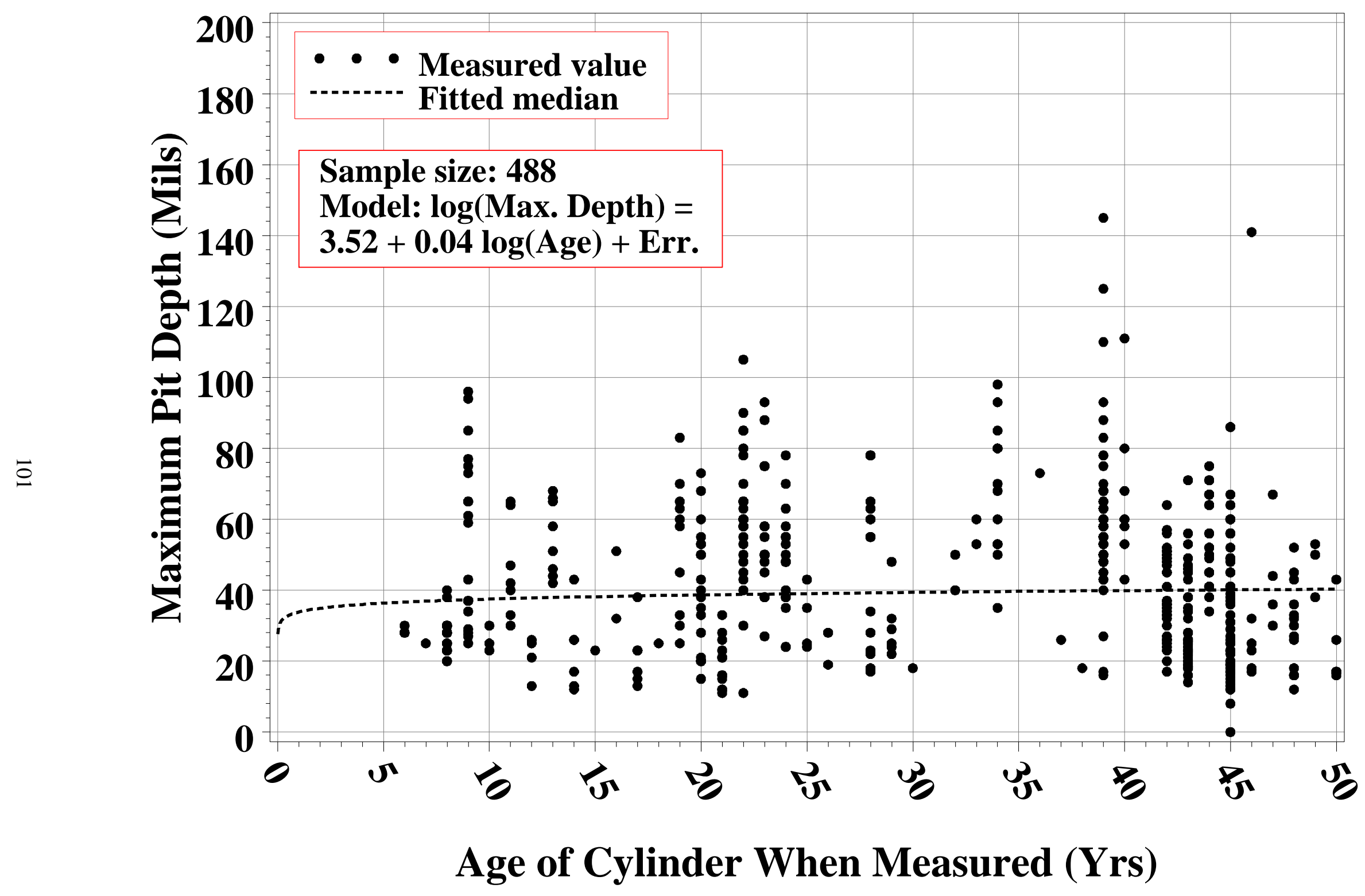

Figure 12. Maximum pit depth estimates for ETTP/PGDP/PORTS thick bottom cylinders. 


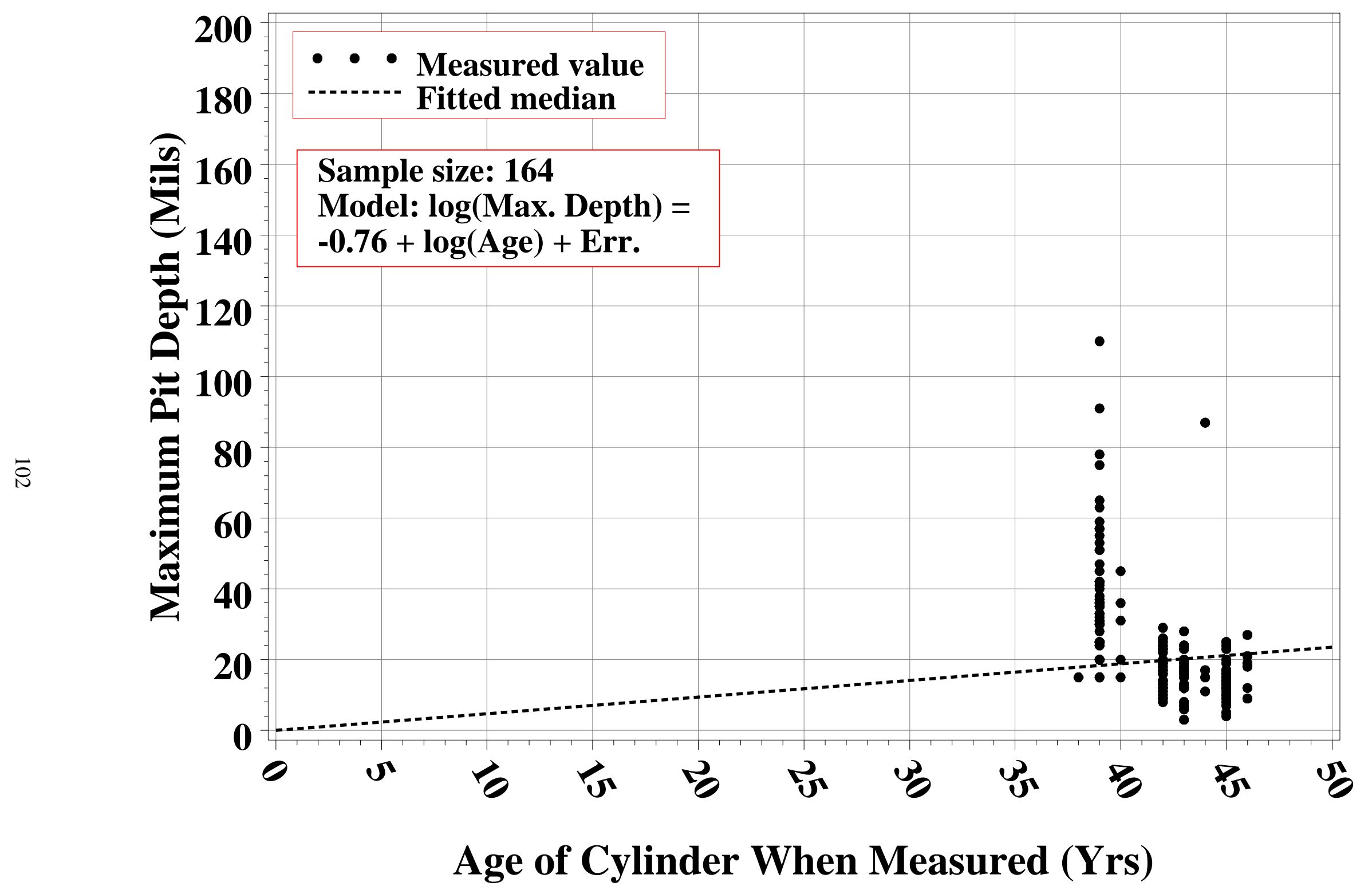

Figure 13. Maximum pit depth estimates for ETTP/PGDP/PORTS thin skirted top cylinders. 


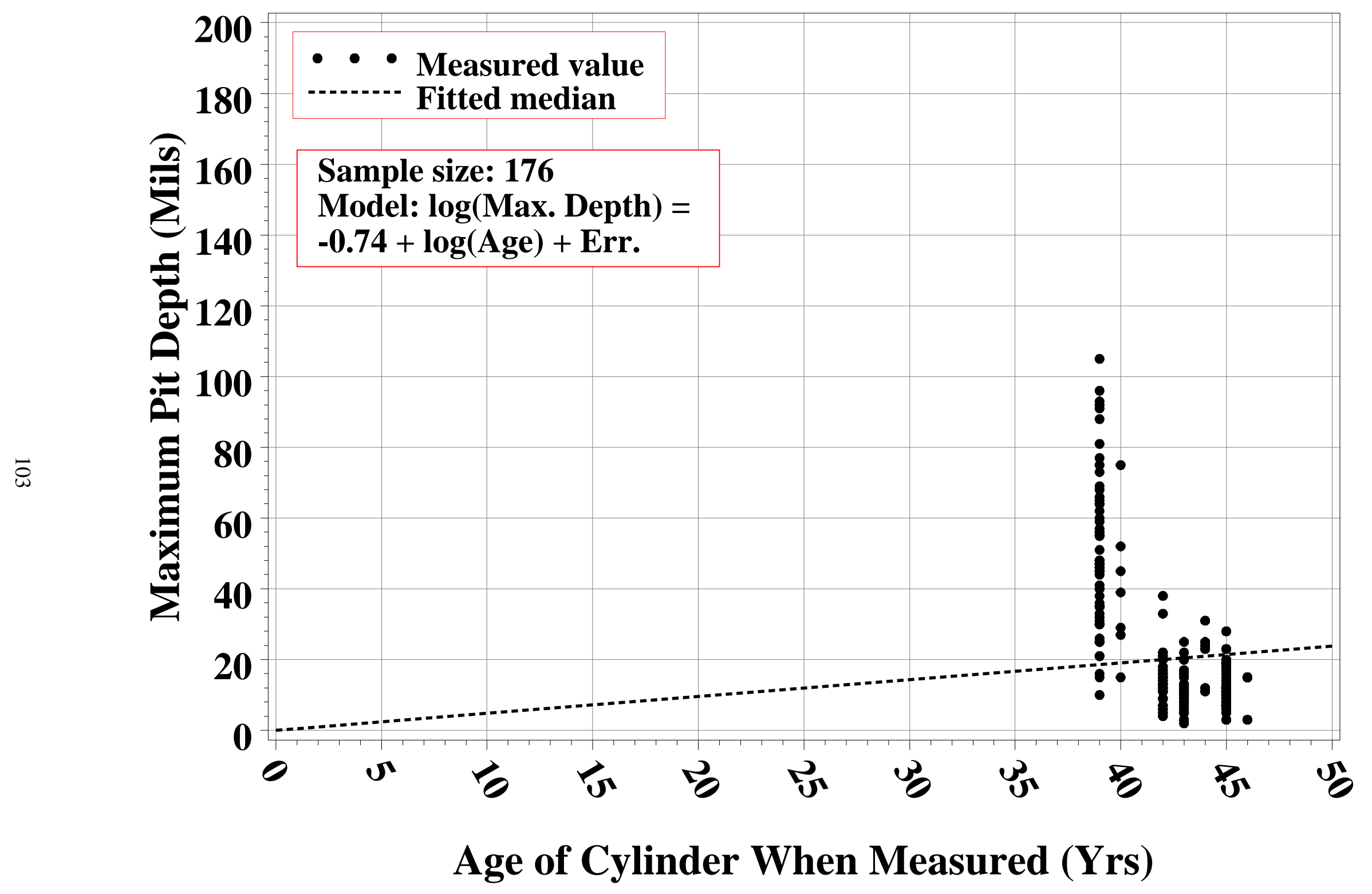

Figure 14. Maximum pit depth estimates for ETTP/PGDP/PORTS thin skirted btm cylinders. 


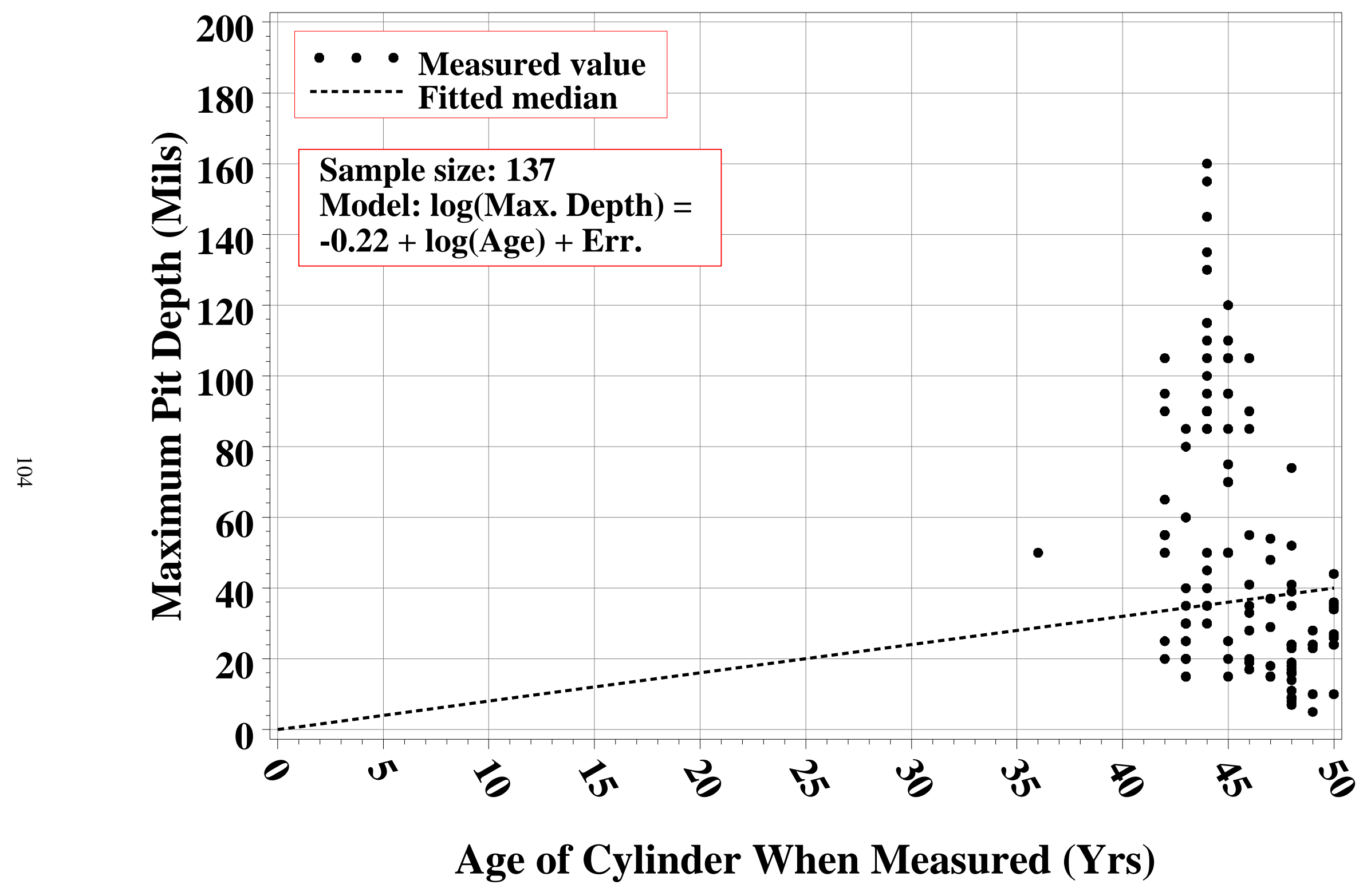

Figure 15. Maximum pit depth estimates for ETTP/PGDP/PORTS thick skirted cylinders. 


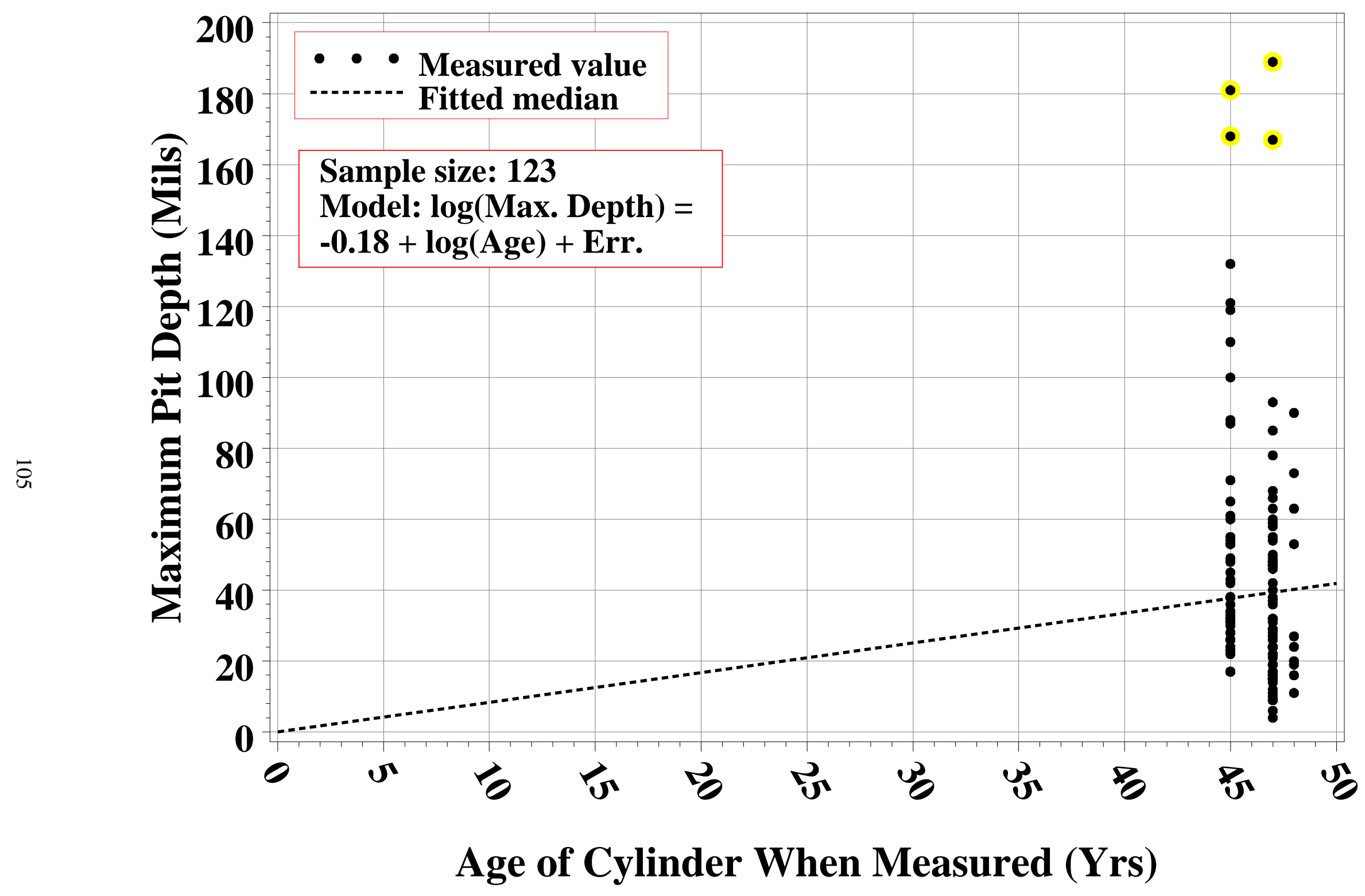

Figure 16. Maximum pit depth estimates for ETTP/PGDP/PORTS 1/2" (30" dia.) top cylinders. 


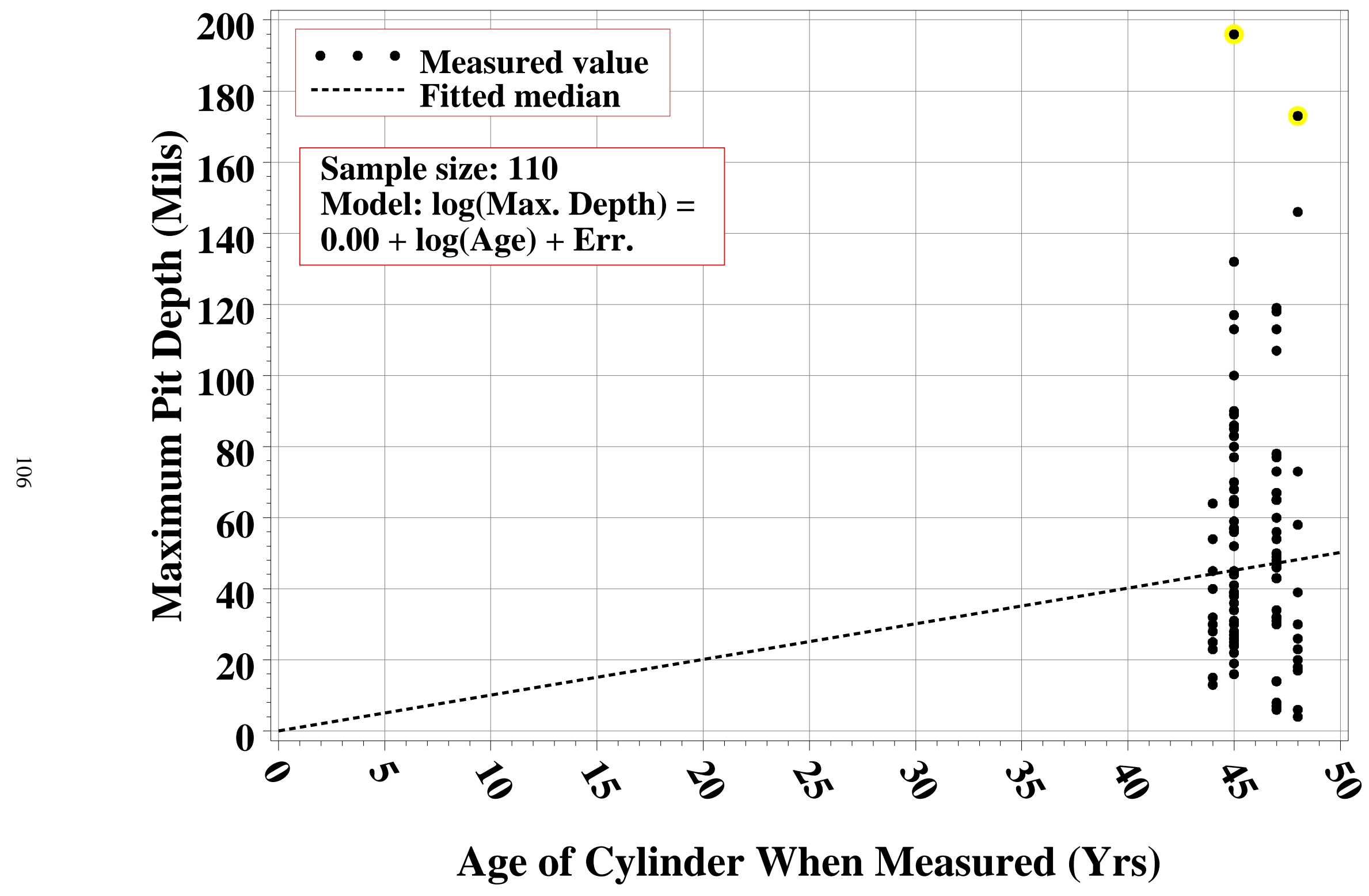

Figure 17. Maximum pit depth estimates for ETTP/PGDP/PORTS 1/2" (30" dia.) btm cylinders. 

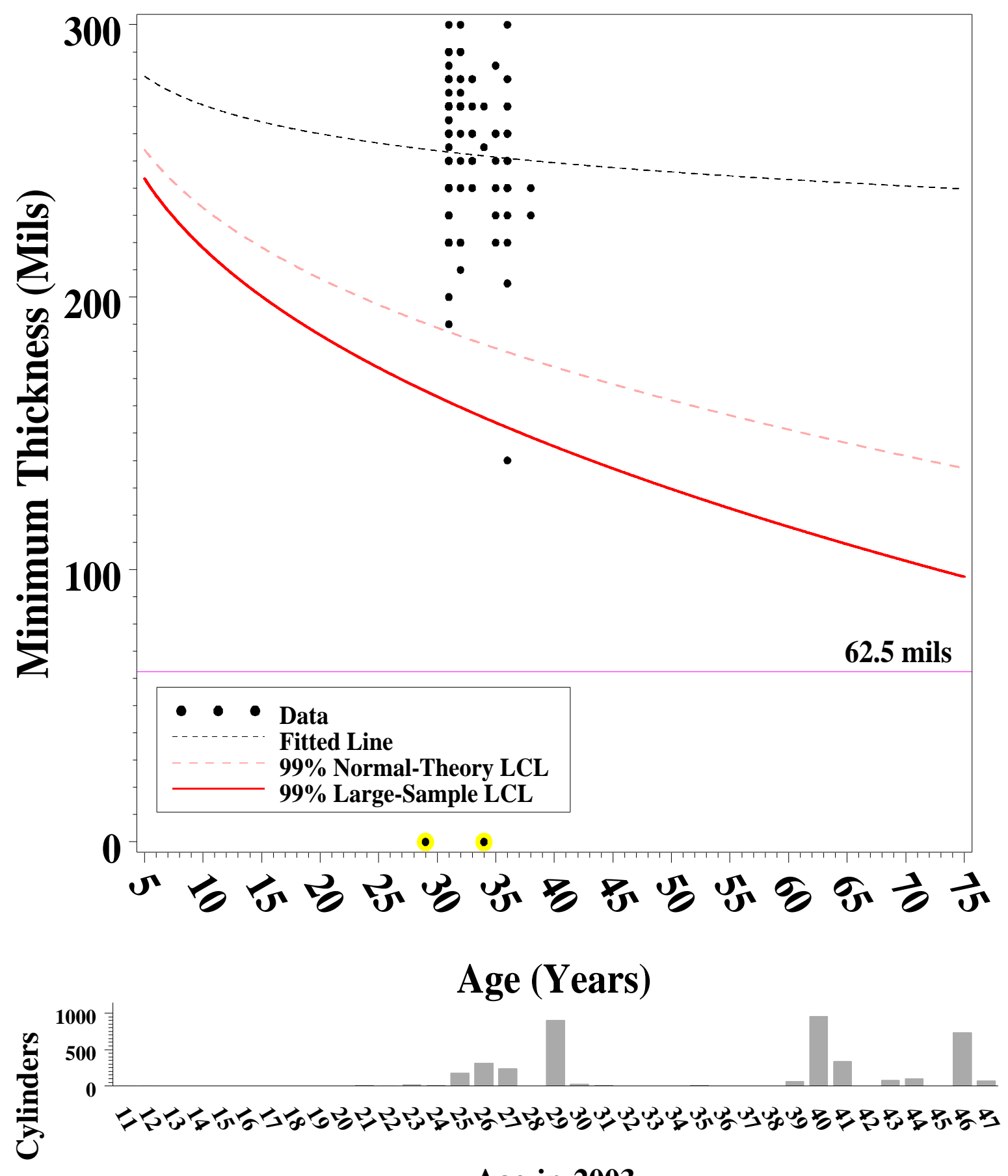

Age-in-2003

Figure 18. Minimum thicknesses (top) and age distribution (below) for ETTP thin (P-scan data) cylinders. Points with corresponding regression residuals below $\mathbf{- 2 0}$ are highlighted (yellow) in Figures 18-36, and those points are excluded from Table 10B. 

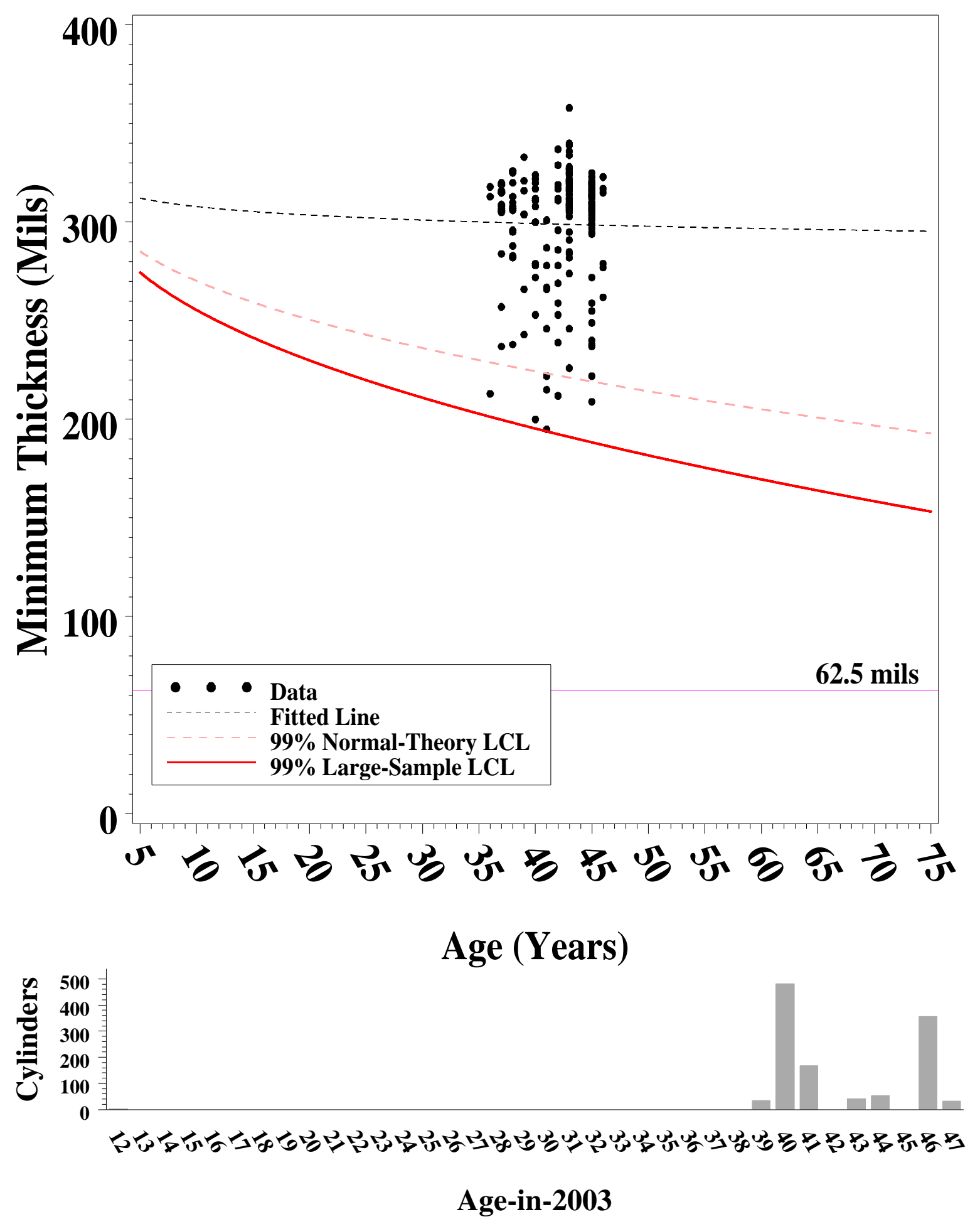

Figure 19. Minimum thicknesses (top) and age distribution (below) for ETTP thin, K-yard bottom cylinders. 

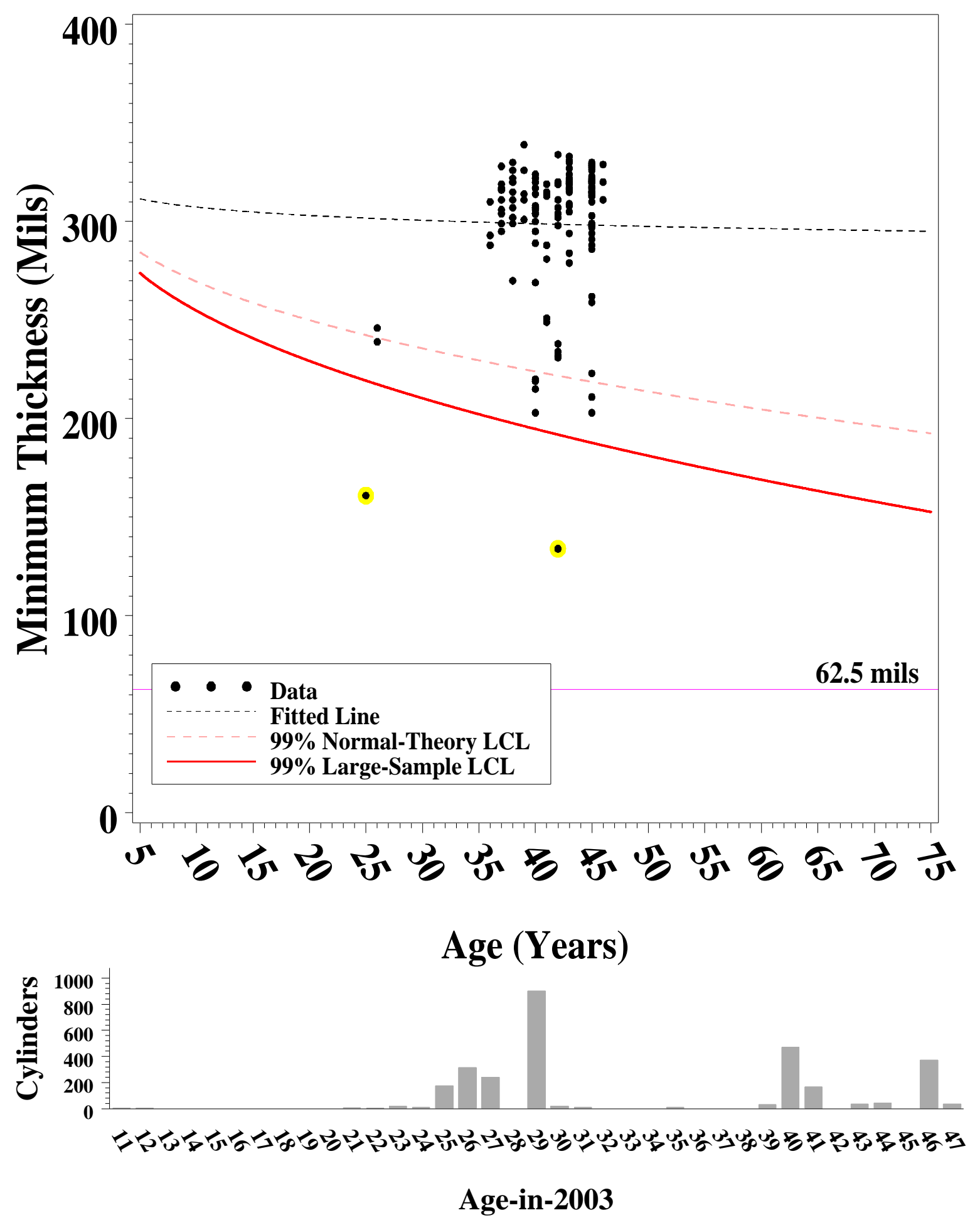

Figure 20. Minimum thicknesses (top) and age distribution (below) for ETTP thin, except K-yard bottom cylinders. 

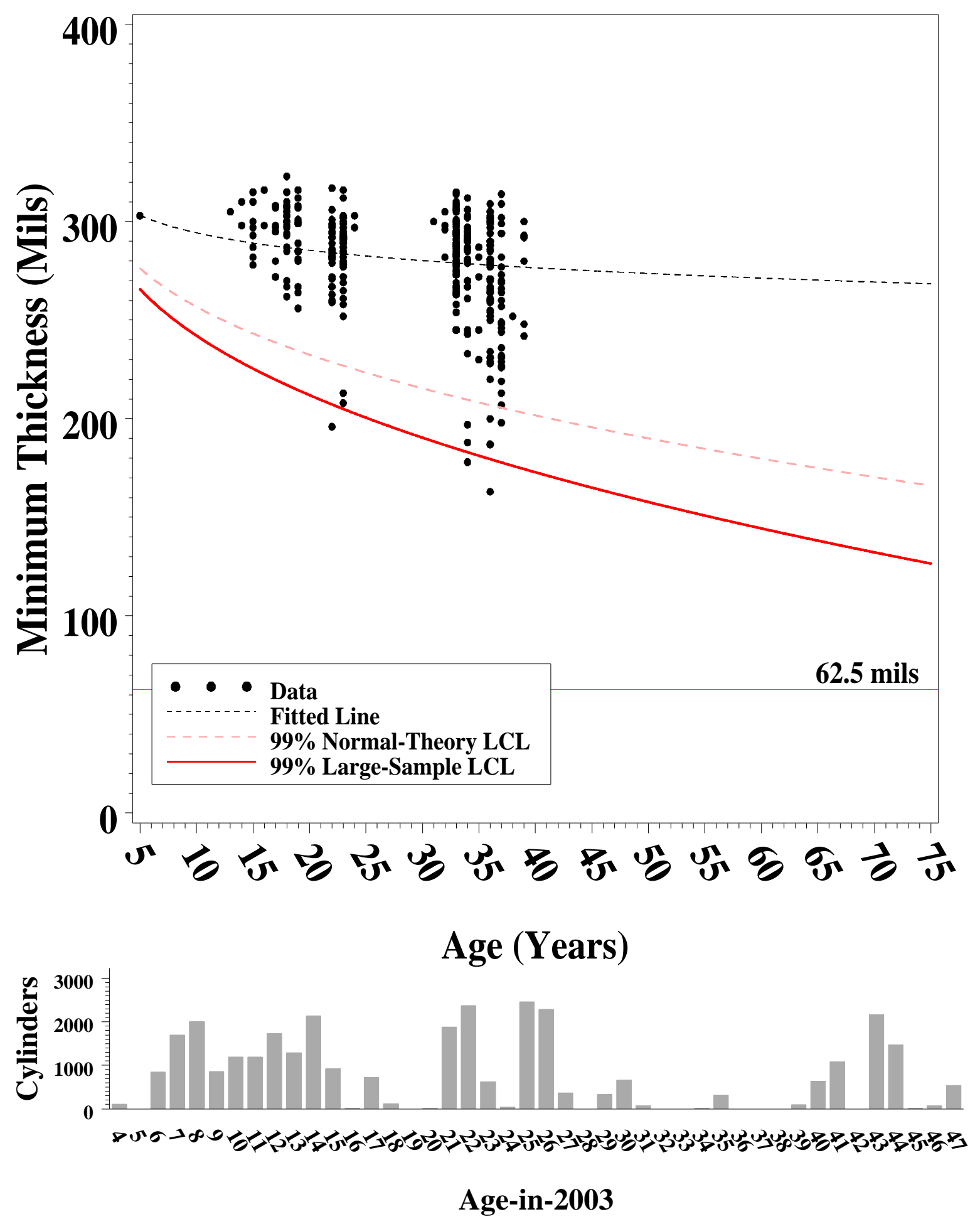

Figure 21. Minimum thicknesses (top) and age distribution (below) for PGDP thin (P-scan data) cylinders. 

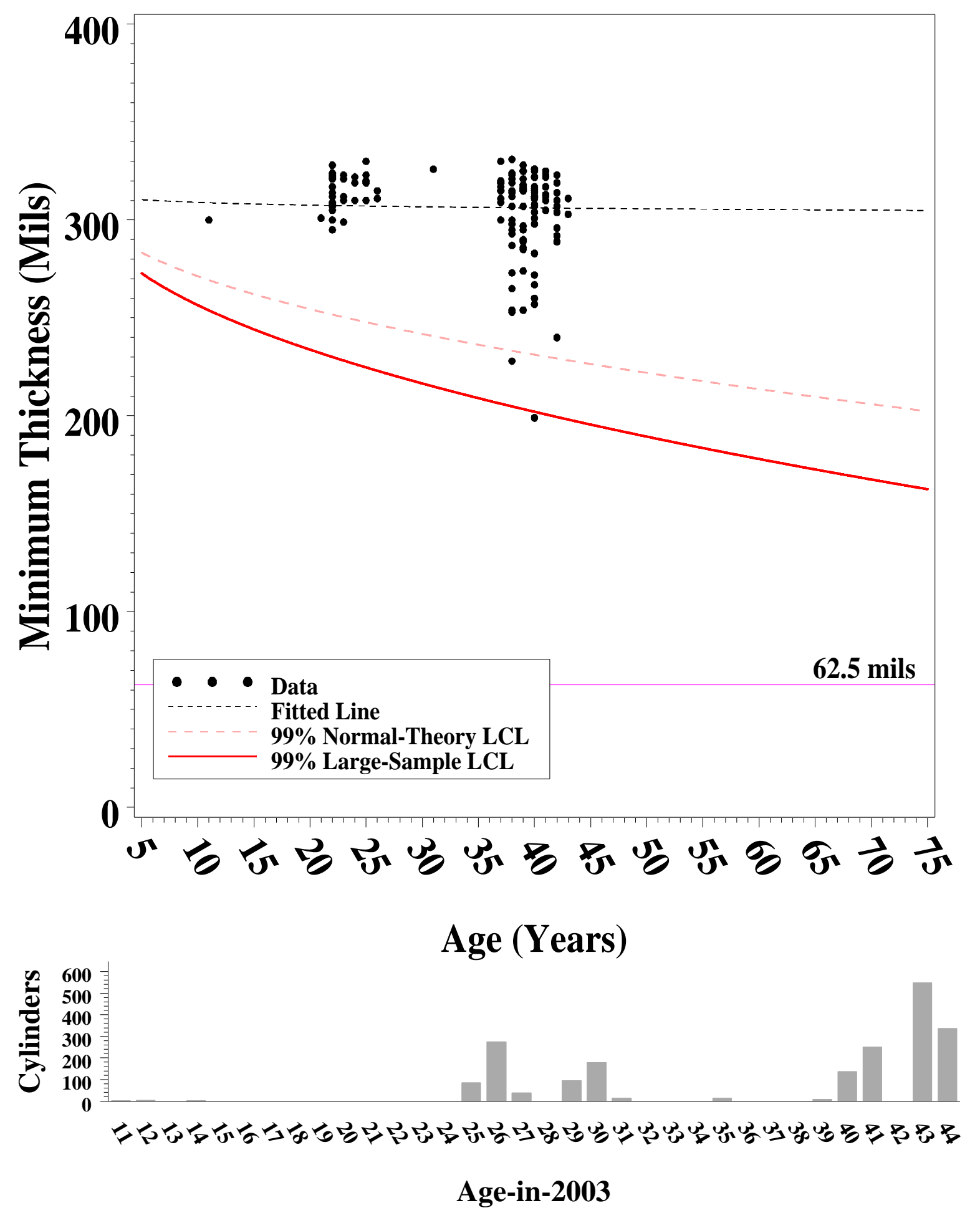

Figure 22. Minimum thicknesses (top) and age distribution (below) for PGDP thin bottom, former G-yard cylinders. 

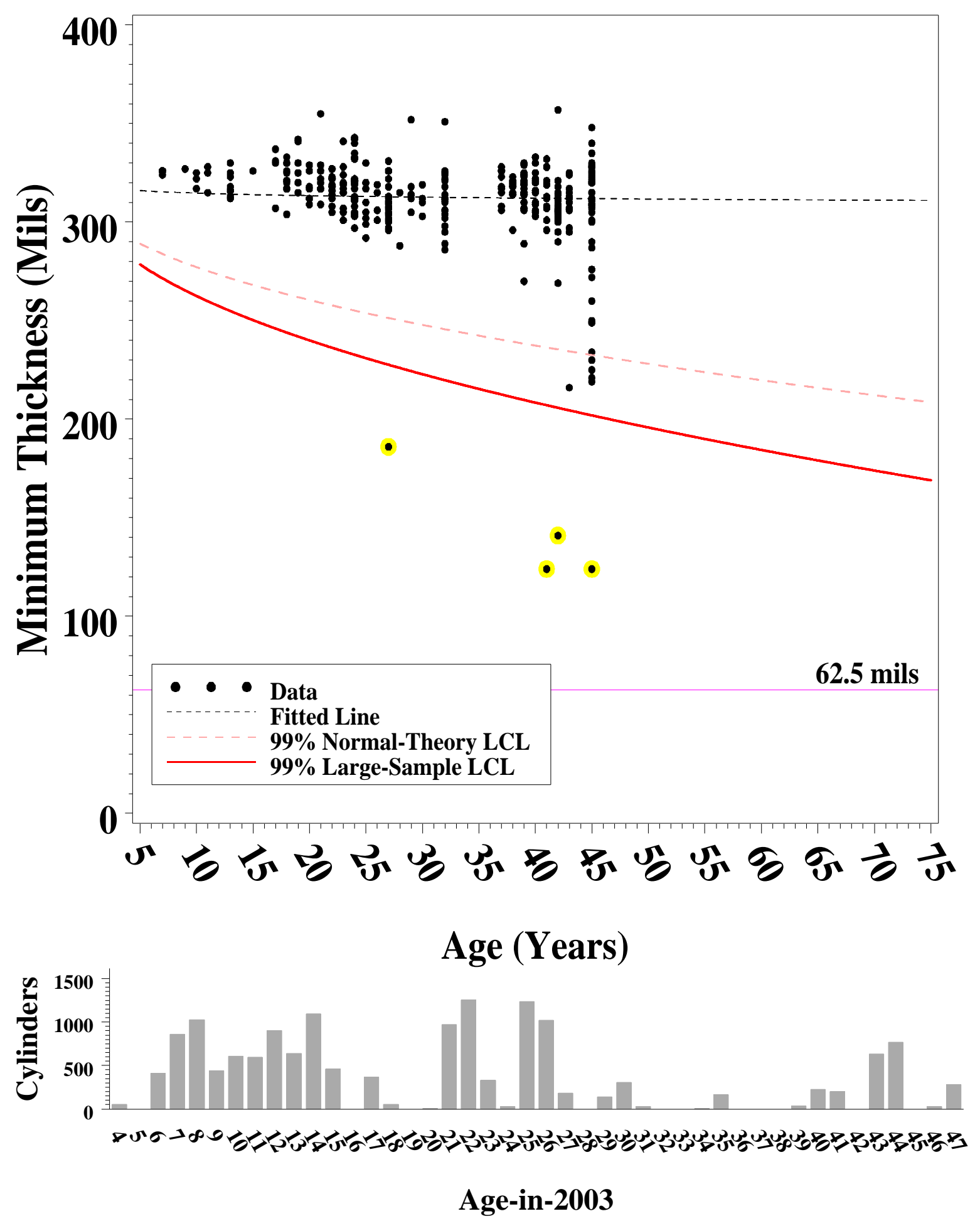

Figure 23. Minimum thicknesses (top) and age distribution (below) for PGDP thin btm, excpt fmr G-yard cylinders. 

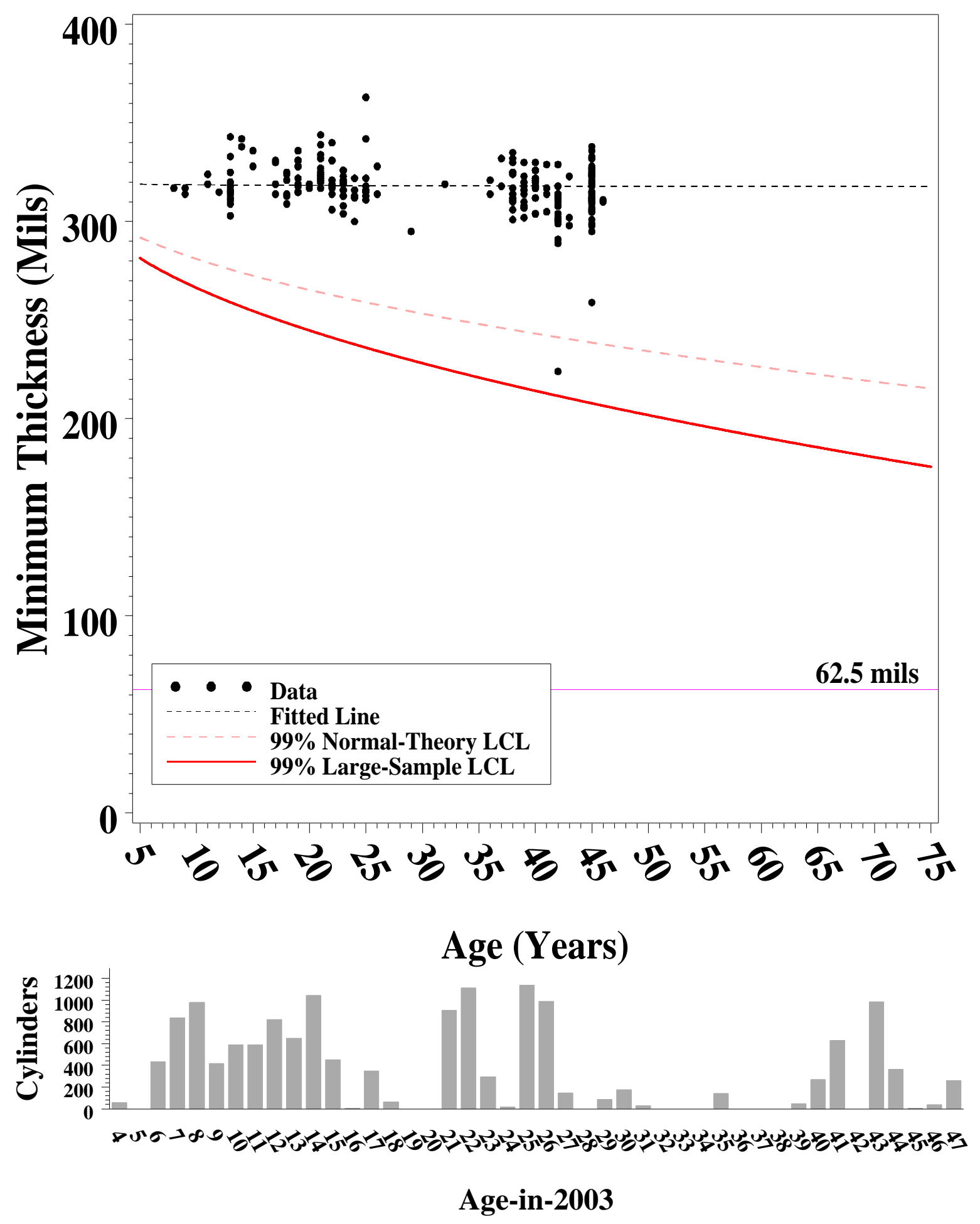

Figure 24. Minimum thicknesses (top) and age distribution (below) for PGDP thin top cylinders. 

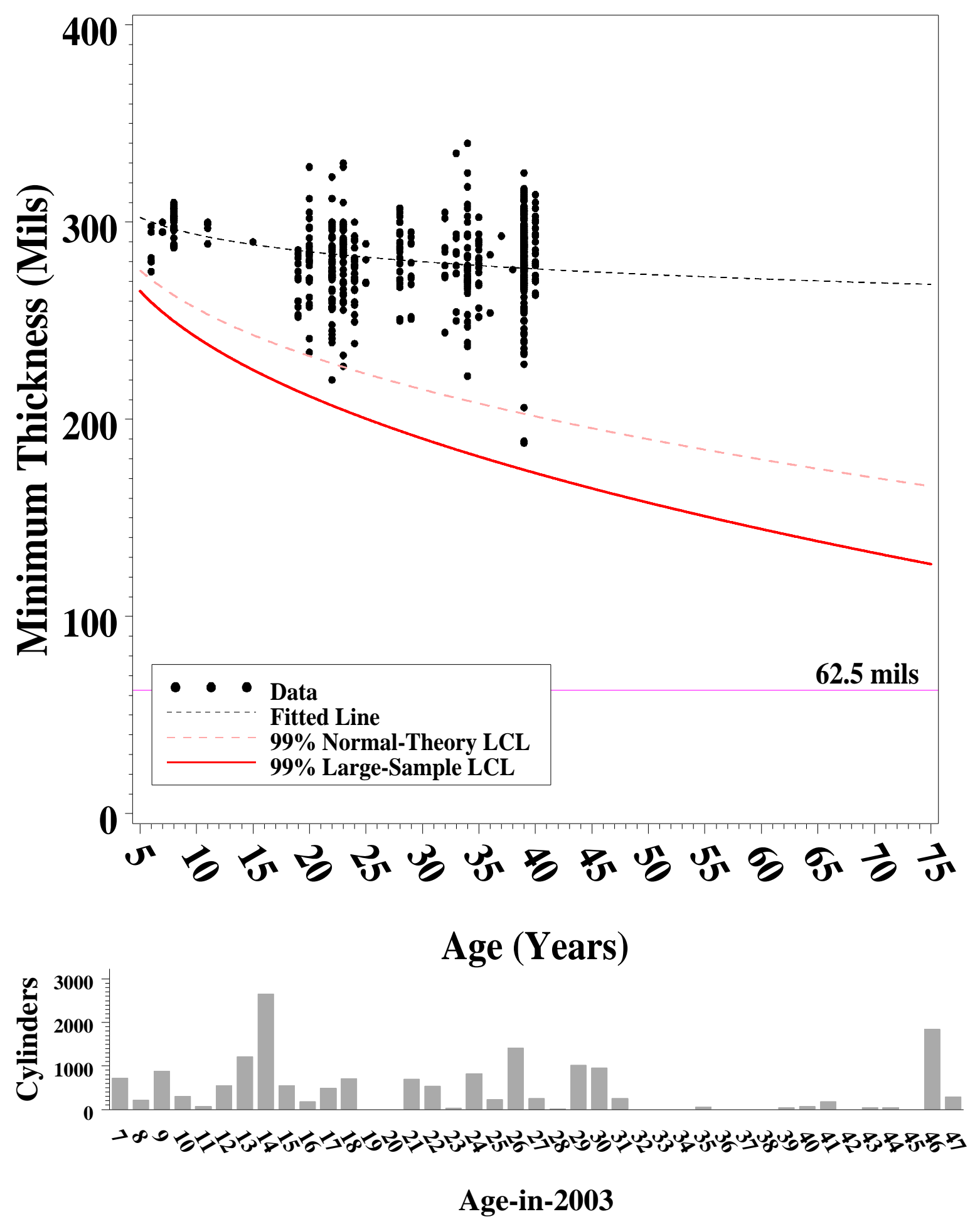

Figure 25. Minimum thicknesses (top) and age distribution (below) for PORTS thin (P-scan data) cylinders. 

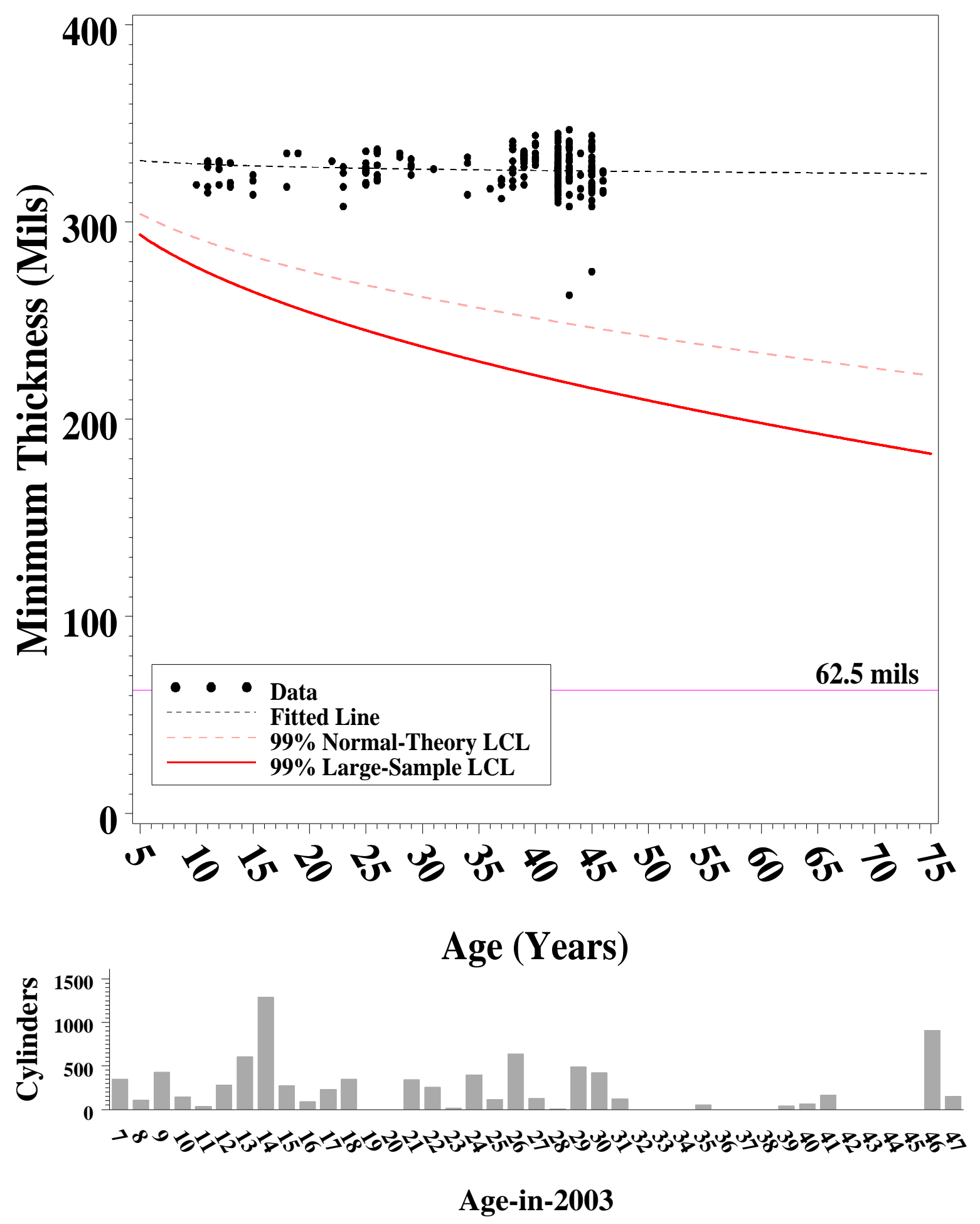

Figure 26. Minimum thicknesses (top) and age distribution (below) for PORTS thin top cylinders. 

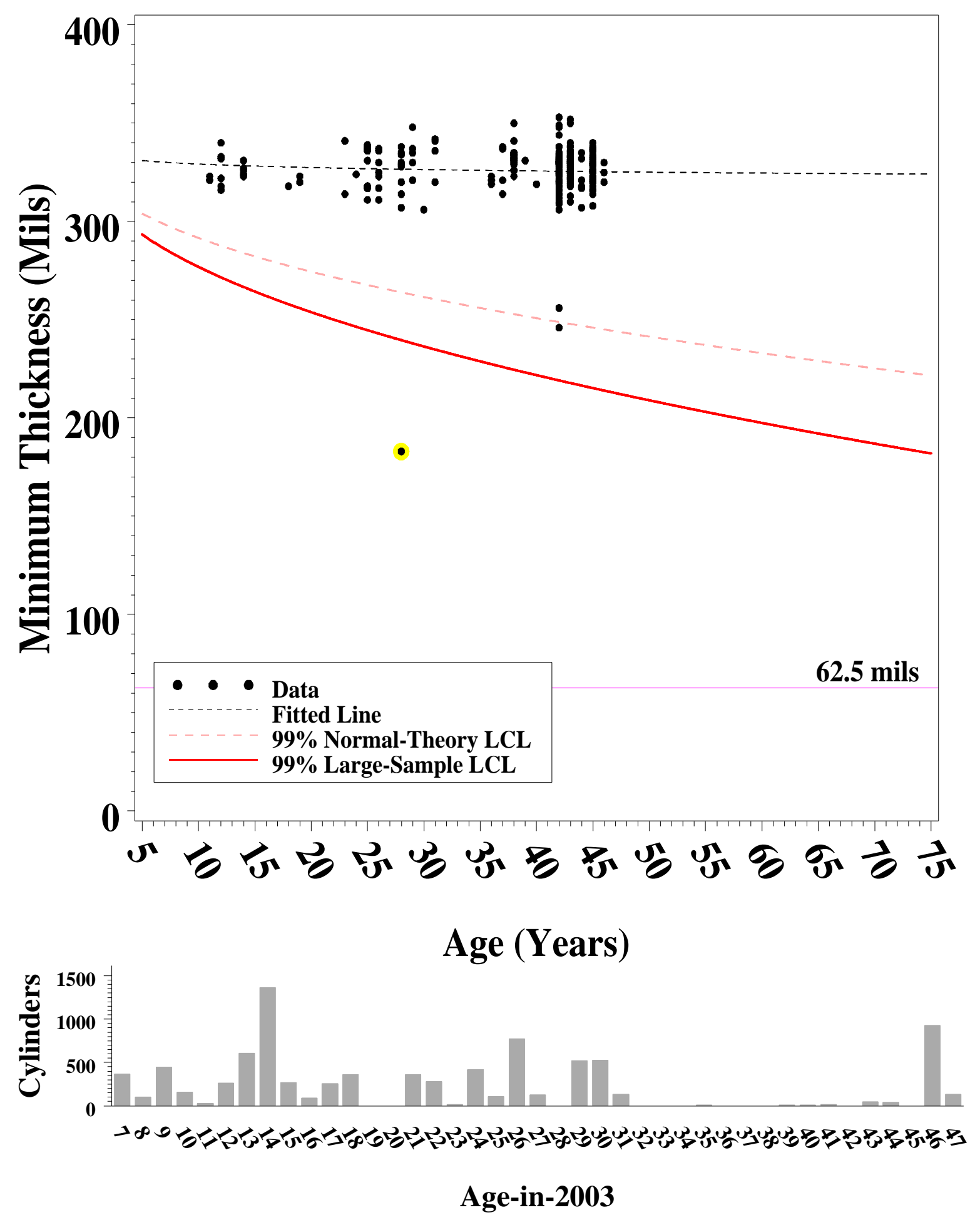

Figure 27. Minimum thicknesses (top) and age distribution (below) for PORTS thin bottom cylinders. 

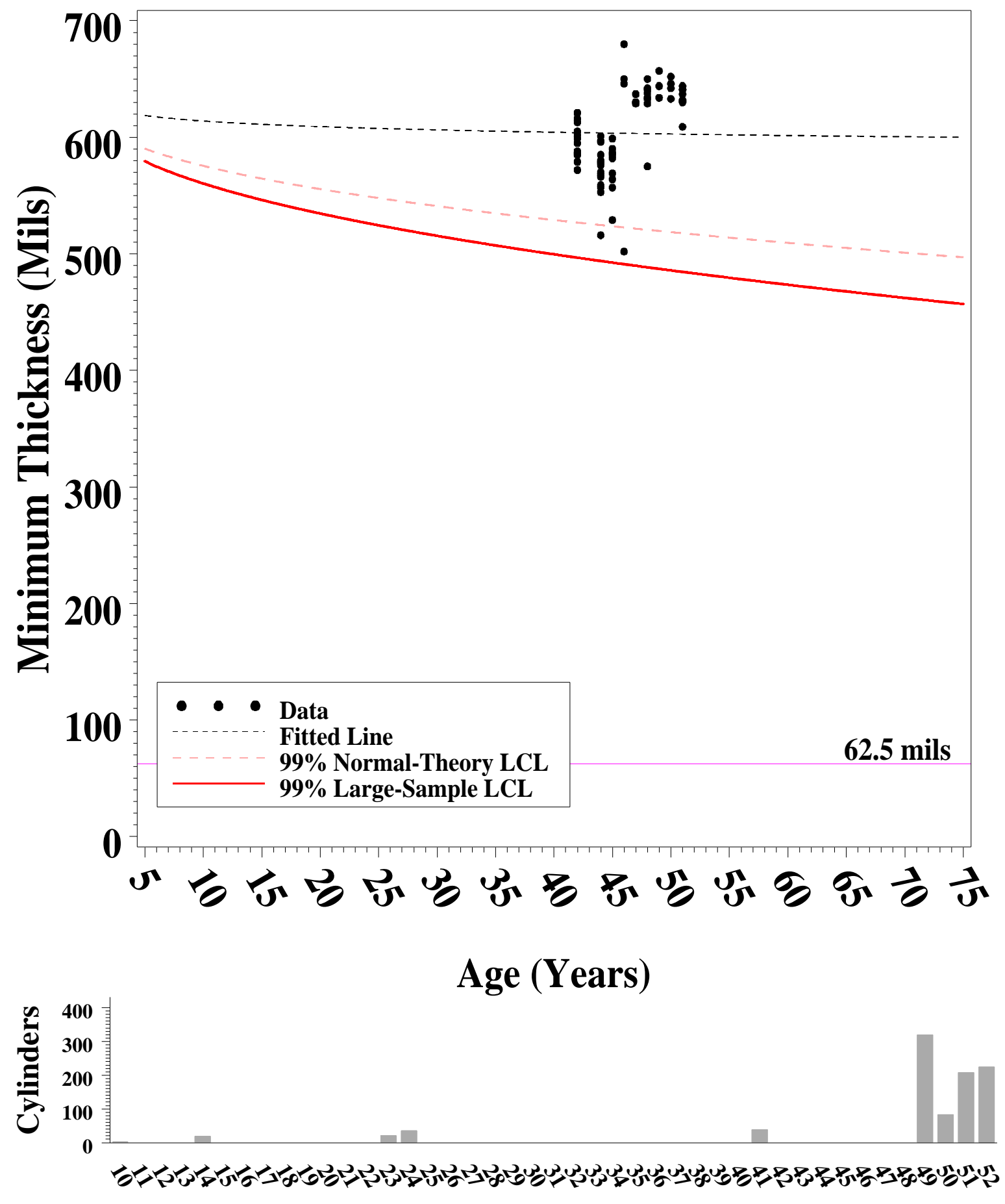

Age-in-2003

Figure 28. Minimum thicknesses (top) and age distribution (below) for ETTP/PGDP/PORTS thick top cylinders. 

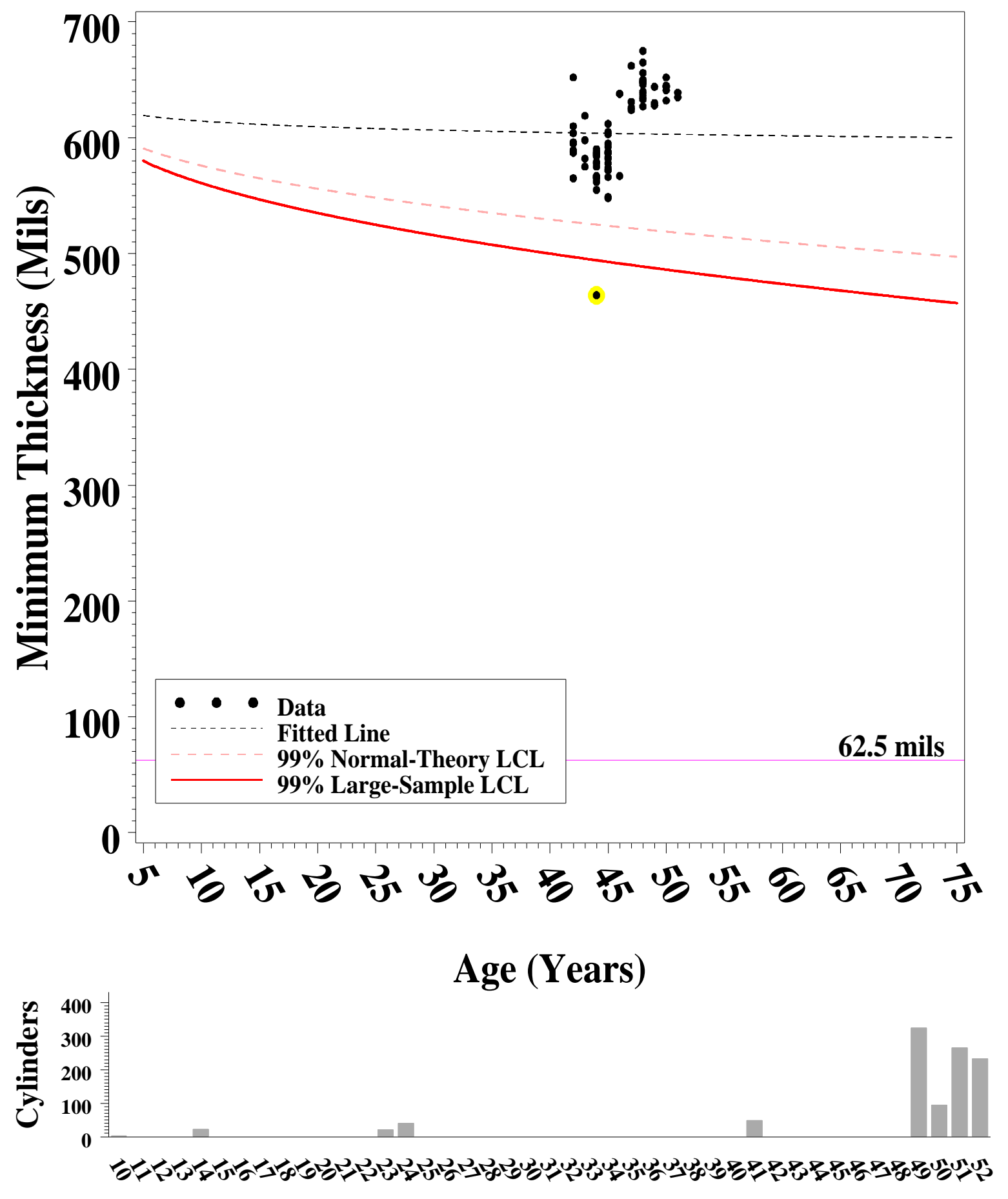

Age-in-2003

Figure 29. Minimum thicknesses (top) and age distribution (below) for ETTP/PGDP/PORTS thick bottom cylinders. 

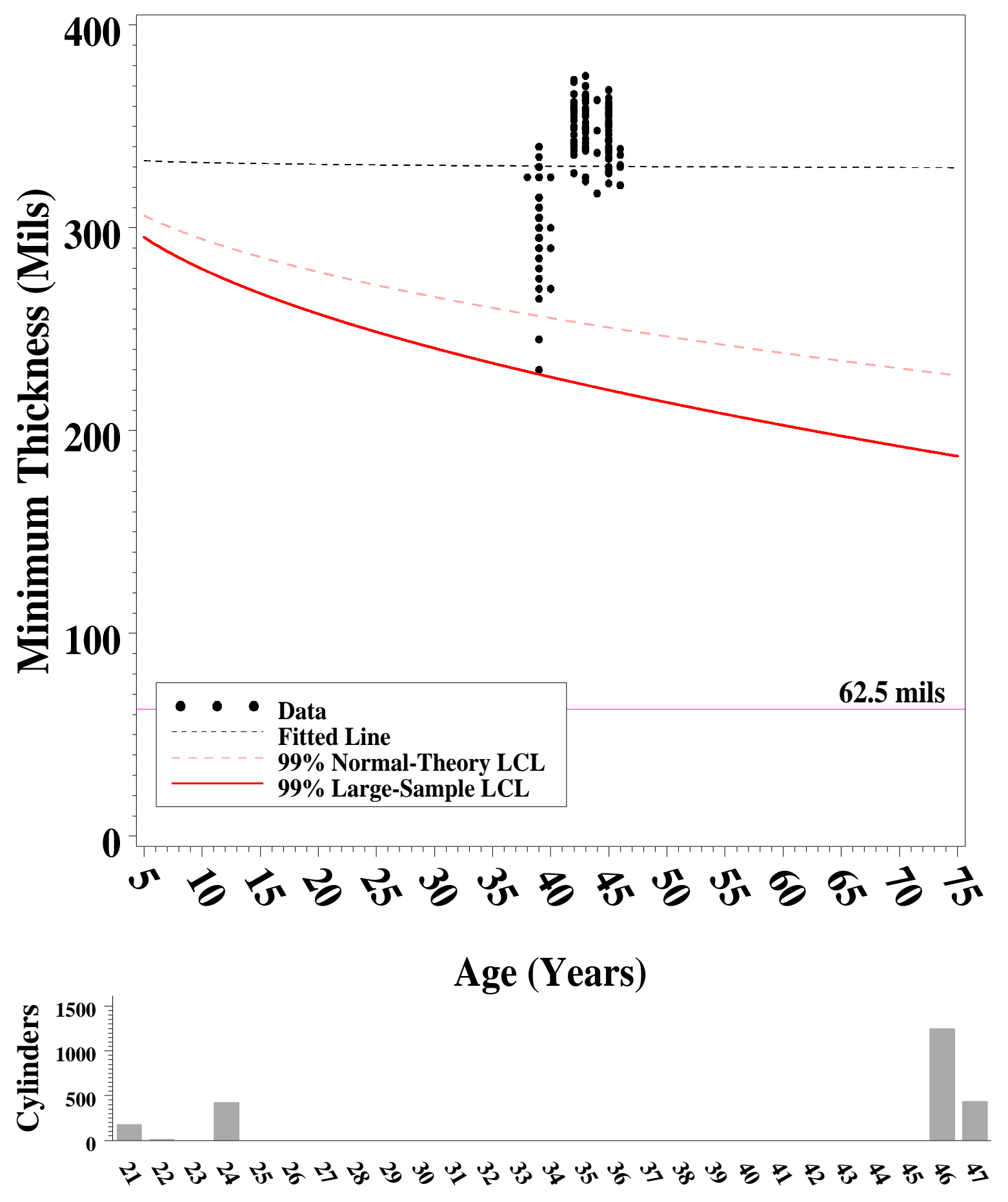

Age-in-2003

Figure 30. Minimum thicknesses (top) and age distribution (below) for ETTP/PGDP/PORTS thin skirted top cylinders. 

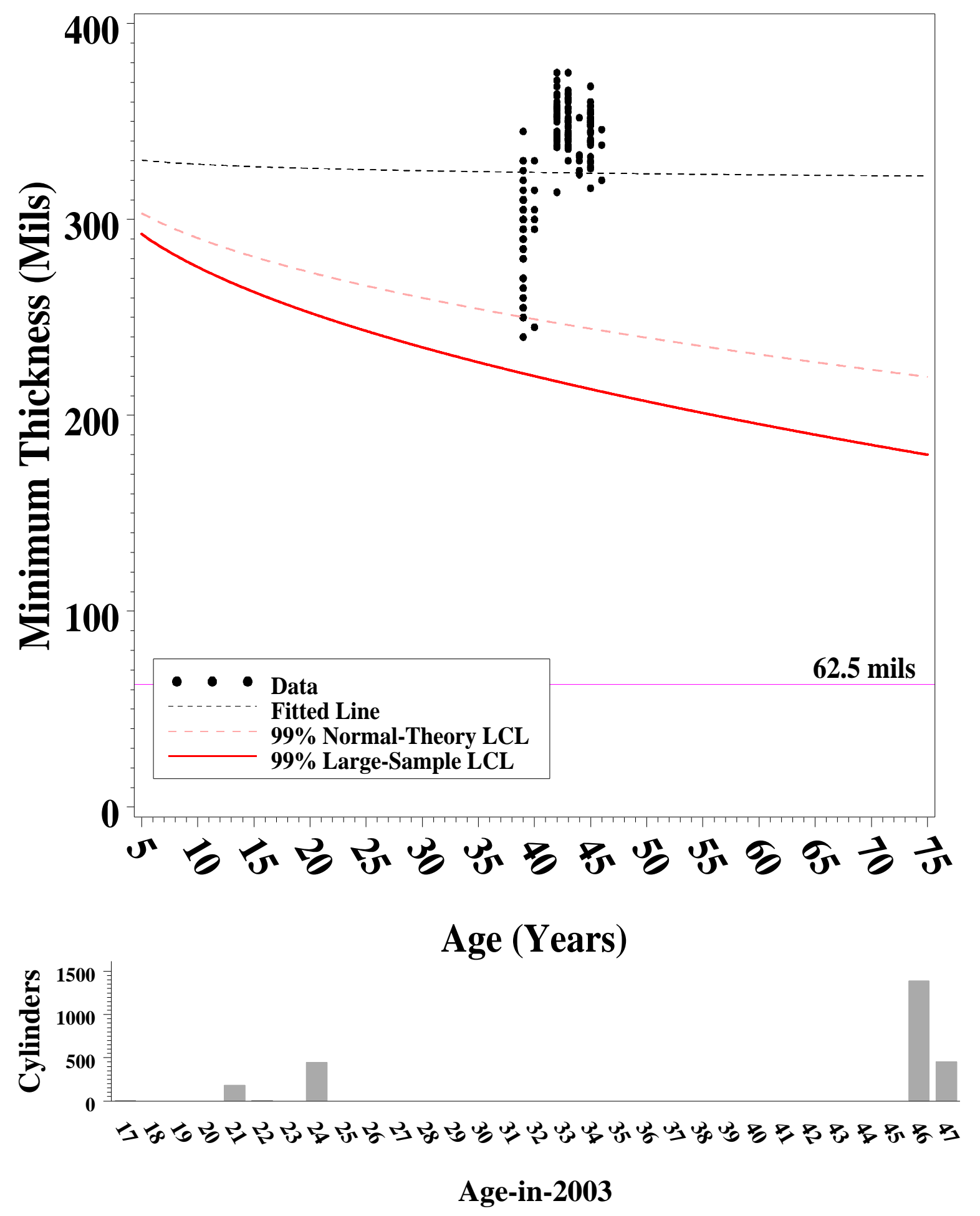

Figure 31. Minimum thicknesses (top) and age distribution (below) for ETTP/PGDP/PORTS thin skirted btm cylinders. 

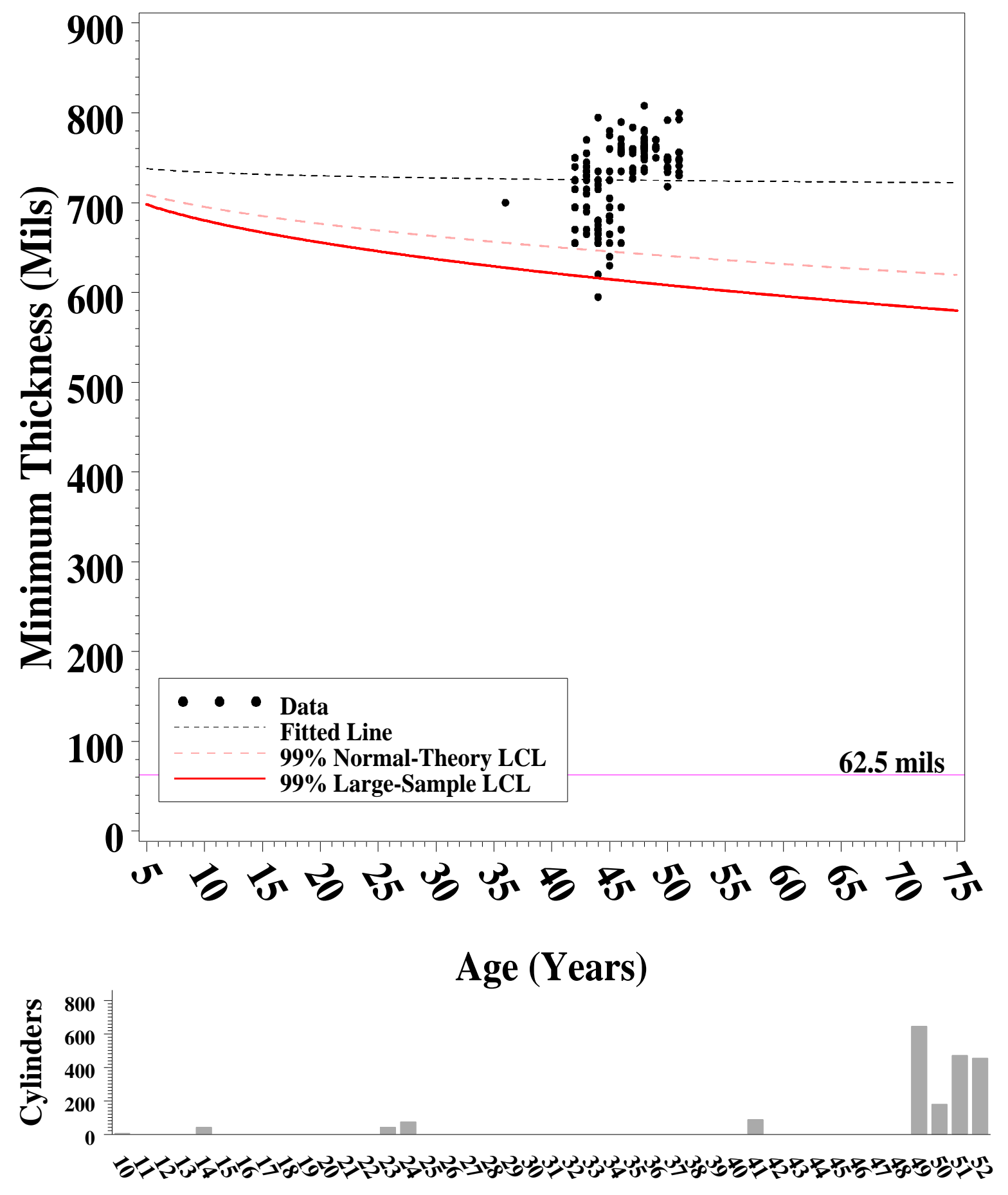

Age-in-2003

Figure 32. Minimum thicknesses (top) and age distribution (below) for ETTP/PGDP/PORTS thick skirted cylinders. 

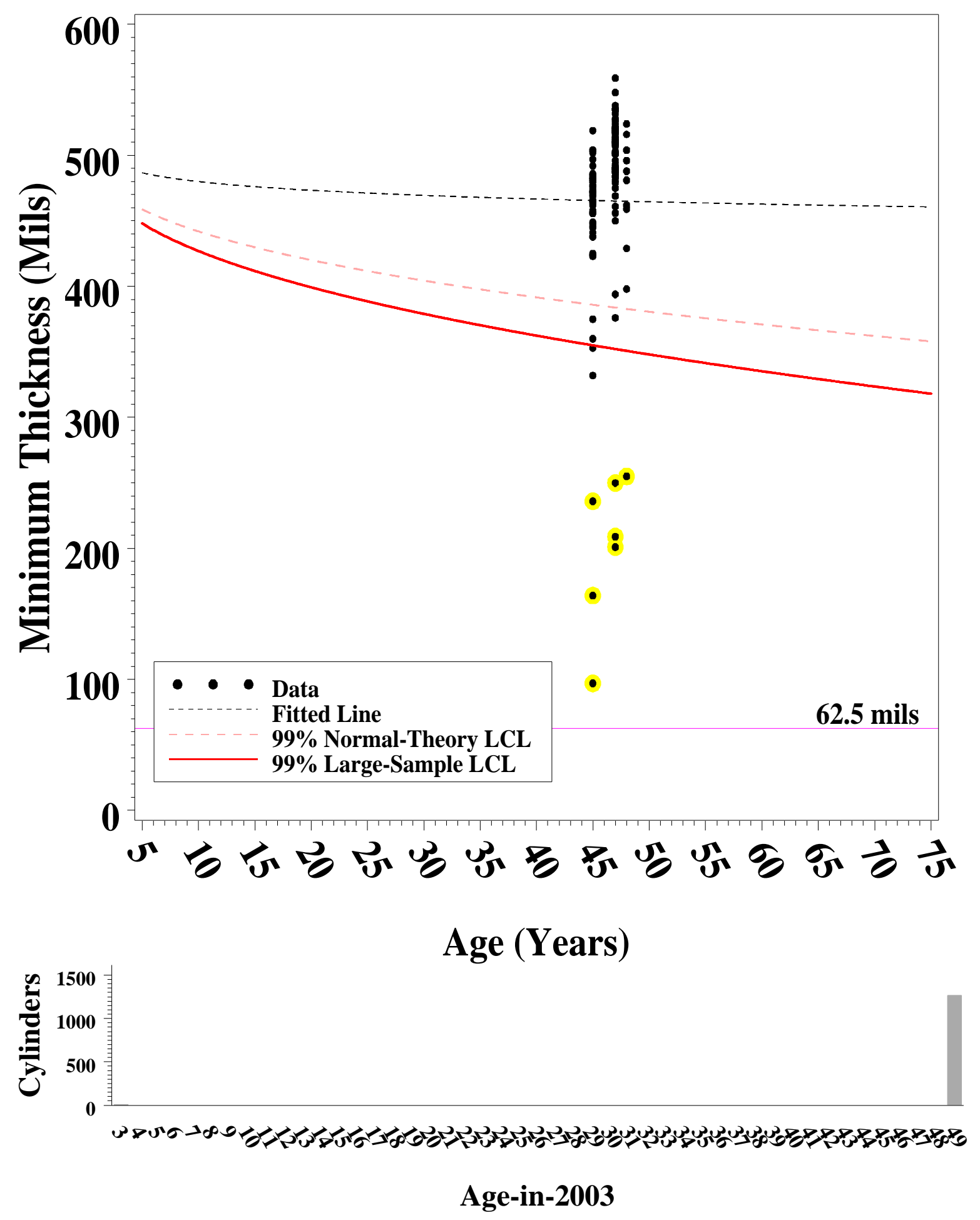

Figure 33. Minimum thicknesses (top) and age distribution (below) for ETTP/PGDP/PORTS 1/2" (30" dia.) top cylinders. 

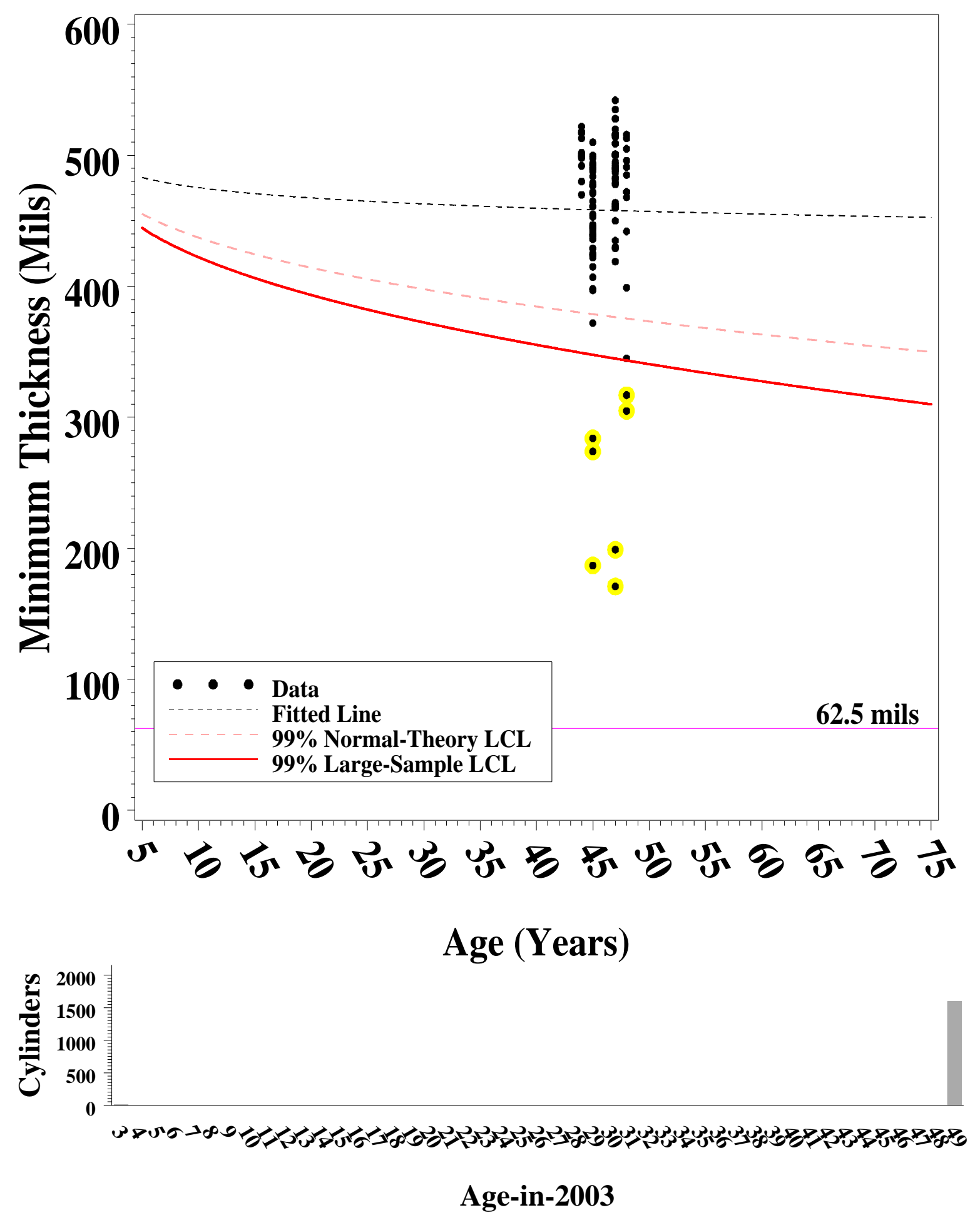

Figure 34. Minimum thicknesses (top) and age distribution (below) for ETTP/PGDP/PORTS 1/2" (30" dia.) btm cylinders. 


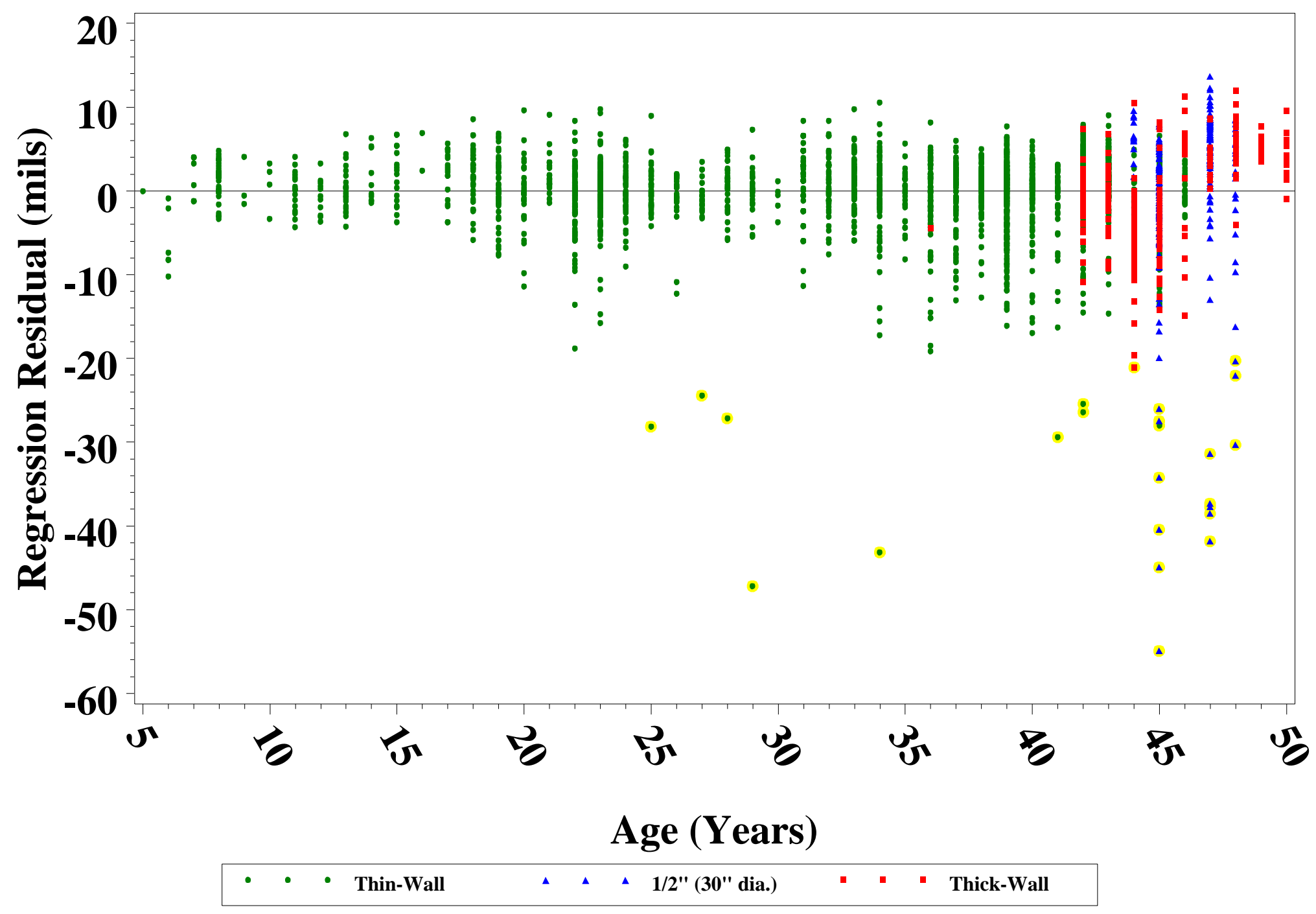

Figure 35. Residuals from minimum thickness regression. Residuals below -20 mils are highlighted (yellow) and corresponding cylinders are not used to compute Table 14B. 


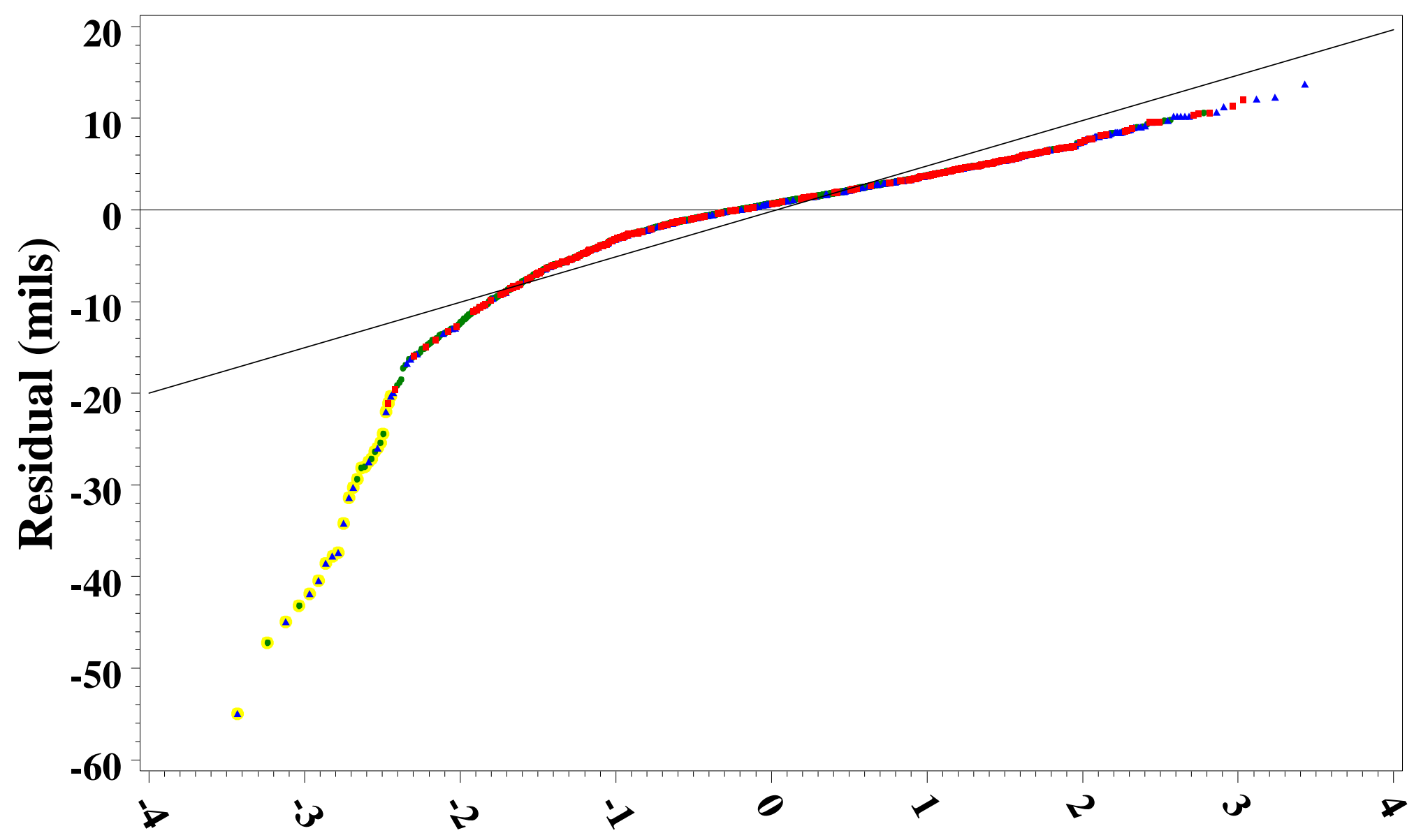

Normal Score

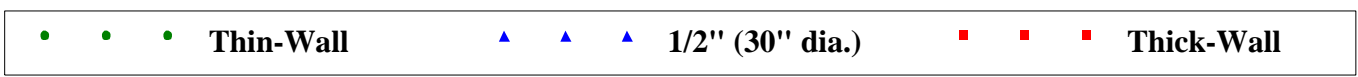

Figure 36. Normal probability plot for the regression residuals. Systematic departures from the straight reference line suggest non-normal data. 


\section{APPENDIX B: PROBABILITY AND CONFIDENCE BOUND CALCULATIONS FOR THE INDIRECT MODEL}

\section{B.1. Cumulative distribution function for the difference of two distributions}

The indirect-model methods discussed in this report are based on the model

$$
M(t) \approx C_{0}-P(t)
$$

where $M(t)$ is the minimum wall thickness at cylinder age t, $P(t)$ is the maximum corrosion depth, and $C_{0}$ is the initial thickness. By the discussion in Section 2, this approximation is conservative in the sense that $M(t) \geq C_{0}-P(t)$. Both $P(t)$ and $C_{0}$ are taken as random, and estimating the number of cylinders that have a minimum thickness below a certain thickness $z$ entails estimating the probability that $C_{0}-P(t)<z$. Since $C_{0}$ and $P(t)$ are both random, this probability is not as straightforward as probabilities for $C_{0}$ and $P(t)$ separately (except for certain special cases, such as when $P(t)$ and $C_{0}$ are both normally distributed, in which case their difference is also normal). In this section, the method is developed for calculating probabilities about $C_{0}-P(t)$.

General Formula. Let $Z=X-Y$, where $X$ and $Y \geq 0$ are independent random variables, with cumulative distribution functions (cdfs) $\mathrm{F}$ and $\mathrm{G}$ respectively. Then

$$
\operatorname{Prob}(Z<z)=\operatorname{Prob}(X-Y<z)=\operatorname{Prob}(Y>X-z)=\int_{x=z}^{\infty} \int_{\{y>x-z\}} d G(y) d F(x)=\int_{x=z}^{\infty}(1-G(x-z)) d F(x) .
$$

Therefore

$$
\operatorname{Prob}(Z<z)=\int_{x=z}^{\infty}(1-G(x-z)) d F(x)
$$

For $G$ lognormal with log-scale mean and variance $\mu$ and $\sigma^{2}$, this is

$$
\int_{x=z}^{\infty}\left(1-\Phi\left(\frac{\log (x-z)-\mu}{\sigma}\right)\right) d F(x)=\int_{x=z}^{\infty} \Phi\left(\frac{\mu-\log (x-z)}{\sigma}\right) d F(x) .
$$

where $\Phi$ denotes the standard normal cumulative distribution function.

Integral (B.1) can be evaluated using the adaptive quadrature method described in Burden and Faires (1989). With this method, subintervals are determined so that the integral is approximated with the desired accuracy using Simpson's rule on each subinterval. (This method is generally faster than simpler integration methods to achieve the same accuracy because the ultimate subdivision that is used need not be uniformly spaced over the entire interval of integration; the subintervals can be selected on the basis of the desired accuracy and the variability of the function to be integrated.) 
Application. In this report, $F$ is the cdf for the initial thickness $C_{0}$, which has a truncated normal distribution, and $G$ is the cdf for the pit depth $P(t)$ at a fixed time $t$, which is lognormal with mean of the logarithm of the values of $\mu(t)$ and standard deviation of the logarithm of the values of $\sigma$.

By the formula above

$$
\operatorname{Prob}\left(C_{0}-P(t)<z\right)=\int_{x=z}^{\infty} \Phi\left(\frac{\mu(t)-\log (x-z)}{\sigma}\right) d F(x),
$$

where the cdf $F$ is truncated normal: for an interval $[a, b]$ and mean $\mu^{\prime}$ and standard deviation $\sigma^{\prime}$,

$$
F(x)= \begin{cases}0 & \text { if } x<a \\ \frac{N\left(x ; \mu^{\prime}, \sigma^{\prime}\right)-N\left(a ; \mu^{\prime}, \sigma^{\prime}\right)}{N\left(b ; \mu^{\prime}, \sigma^{\prime}\right)-N\left(a ; \mu^{\prime}, \sigma^{\prime}\right)} & \text { if } a<x<b \\ 1 & \text { if } x>b\end{cases}
$$

where $N(x ; m, s)=\Phi((x-m) / s)$.

\section{B.2. Calculation of Upper Confidence Limits}

In the methods used in this report, the maximum pit depth $P(t)$ is modeled using a lognormal distribution, with either $P(t) \sim \log \left(\mu_{L}, \sigma_{L}\right) \times t$ (slope set to 1$)$ or $P(t) \sim \log \left(\log (A)+n \log (t), \sigma_{L}\right)$, and the parameters are fit with the available UT data. The expected number of cylinders with a minimum thickness below a certain thickness $z$ by a given time $T$ is calculated as

$$
\sum_{i} \operatorname{Prob}\left(C_{0}-P\left(t_{i}\right)<z\right) \times\left\{\text { Number of cylinders of age } \mathrm{t}_{\mathrm{i}} \text { at time } T\right\}
$$

where the sum is over all age classes for the cylinder population of interest.

Given the initial thickness and pit depth distributions, the probabilities in (3) can be estimated using estimates of the initial thickness and pit depth distributions and the numerical approach discussed above. In this subsection confidence limits for (B.2) are developed to account for uncertainty in the estimates of the pit depth distribution. The uncertainty in the initial thickness distribution is assumed to be negligible.

The approach taken to calculating a UCL for the sum (B.2) is based on the Bonferroni inequality, which can be used to determine a value $\alpha$ such that if an upper $100 \alpha \%$ confidence limit is used for each term in the sum, the final sum will be bounded with at least $95 \%$ confidence. However, although expression (3) may have up to 25 terms (i.e., for as many as 25 different ages), the statistical distributions of all of the terms depend on just three parameters - the intercept, slope, and standard deviation from the regression of logdepth on log-age (with uncertainty in the initial thickness distribution assumed negligible). Therefore, joint confidence limits for the pit depth at each age represented in (B.2) can also be computed from joint confidence limits for the three parameters. This suggests that a more efficient use of the Bonferroni approach would be to use it to derive joint confidence limits for the three parameters, rather than joint confidence limits for all of the terms in (B.2). 
Furthermore, a refinement of the three-parameter Bonferroni approach is possible. Joint confidence limits for the intercept and slope can be used to derive joint UCLs for the pit depth log-scale means $\mu\left(t_{i}\right)=a+b$ $\log \left(t_{i}\right)$ for each age $t_{i}$. But joint confidence limits for the intercept and slope imply joint confidence limits for all points on the curve $\mu(t)=a+b \log (t)$, including, for example, points for ages such as $t=10,000$ years or $\mathrm{t}=-10,000$ years. In the cylinder modeling, however, the only confidence limits for points on the regression line that are needed are confidence limits for points corresponding to ages of concern-in the range of about 0 to 75 years. As Figure 37 illustrates, the line that interpolates joint UCLs for the regression line at the endpoints of a range of interest is in fact a joint UCL for all points on the regression line in that range. Because their range is restricted, joint UCLs based on the line restricted to the interval, tend to be tighter than UCLs for the whole line, based on confidence limits for the intercept and slope.

Combining equation (B.1) and expression (B.2) gives

$$
\sum_{i}\left\{\text { Number of cylinders of age } \mathrm{t}_{\mathrm{i}} \text { at time } T\right\} \times \int_{x=z}^{\infty} \Phi\left(\frac{\mu\left(t_{i}\right)-\log (x-z)}{\sigma}\right) d F(x)
$$

for the number of cylinders at time $\mathrm{T}$ for which the thickness criterion $\mathrm{z}$ is violated. It is straightforward to show that expression (B.3) is increasing in each $\mu\left(\mathrm{t}_{\mathrm{i}}\right)$. Therefore, for any given $\sigma$, a UCL for expression (B.3) can be obtained by substituting UCLs for the individual $\mu\left(\mathrm{t}_{\mathrm{i}}\right)$. A grid search in $\sigma$, for $\sigma$ in a confidence interval, can the be used to determine an overall UCL for (B.3). A confidence interval for $\sigma$ is can be obtained as follows.

In a lognormal regression with $\mathrm{d}$ degrees of freedom, the mean squared error (MSE) is an unbiased estimate of $\sigma^{2}$, and $\mathrm{d} \times \mathrm{MSE} / \sigma^{2}$ has a chi-square distribution with d degrees of freedom. It follow that $\mathrm{d} \times \mathrm{MSE} / \mathrm{X}_{\alpha}^{2}$ and $\mathrm{d} \times \mathrm{MSE} / \mathrm{X}_{1-\alpha}^{2}$ are upper and LCLs for $\sigma^{2}$, where $\mathrm{X}_{\alpha}^{2}$ and $\mathrm{X}_{1-\alpha}^{2}$ denote the $\alpha$ and 1- $\alpha$ percentiles of the chi-square distribution with $\mathrm{d}$ degrees of freedom. The square roots of the confidence limits are confidence limits for $\sigma$. The Bonferroni procedure can be used to choose $\alpha$ so that these confidence limits are joint with the interval-endpoint confidence limits discussed above.

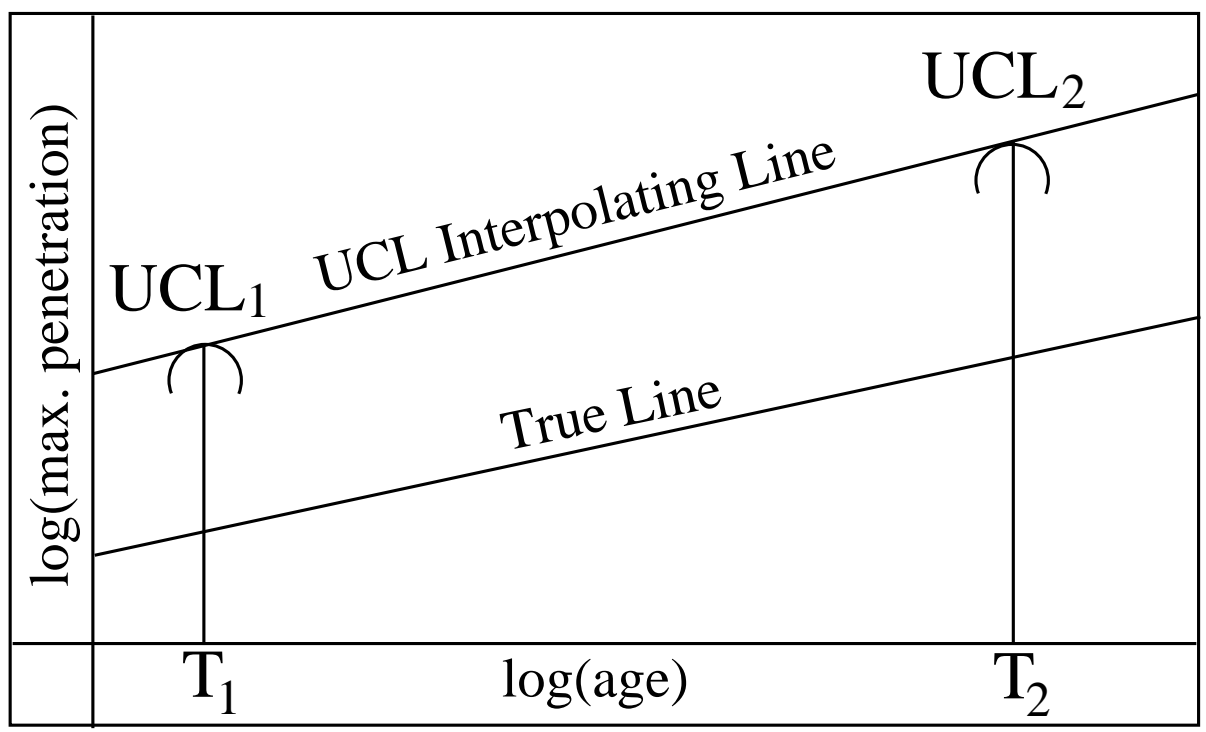

Figure 37. Example of a joint confidence line over an interval, based on two joint UCL's computed at endpoints T1 and T2 of the interval. 


\section{DISTRIBUTION}

1. ORNL Central Research Library

2. ORNL Laboratory Records-OSTI

3. Halen Philpot (Bechtel Jacobs Company)

4. Ted Fox

5. Steve Pawel

6. Carolyn Moser 